$Q K$

5664

SI-Ret

\title{
A Revision of
}

American Velloziaceae

LYMAN B. SMITH

and

EDWARD S. AYENSU

SMITHSONIAN CONTRIBUTIONS TO BOTANY • NUMBER 30 


\section{SERIAL PUBLICATIONS OF THE SMITHSONIAN INSTITUTION}

The emphasis upon publications as a means of diffusing knowledge was expressed by the first Secretary of the Smithsonan Institution. In his formal plan for the Institution, Joseph Henry articulated a program that included the following statement: "It is proposed to publish a series of reports, giving an account of the new discovernes in science, and of the changes made from year to year in all branches of knowledge." This keynote of basic research has been adhered to over the years in the issuance of thousands of titles in serial publications under the Snithsonian imprint, commencing with Smithsonian Contributions to Knowledge in 1848 and continuing with the following active series:

\section{Smithsonian Annals of Flight \\ Smithsonian Contributions to Anthropology \\ Smithsonian Contributions to Astrophysics \\ Smithsonian Contributions to Botany \\ Smithsonian Contributions to the Earth Sciences \\ Smithsonian Contributions to Paleobiology \\ Smithsonian Contributions to Zoology \\ Smithsonian Studies in History and Technology}

In these eries, the Institution publishes original articles and monographs dealing with the research and collections of its several museums and offices and of professional colleagues at other institutions of learning. These papers report newly acquired facts, synoptic interpretations of data, or original theory in specialized fields. These publications are distributed by mailing lists to libraries, laboratories, and other interested institutions and specialists throughout the world. Individual copies may be obtained from the Smithsonian Institution Press as long as stocks are available.

\section{S. Dillon Ripley Secretary \\ Smithsonian Institution}


SMITHSONIAN CONTRIBUTIONS TO BOTANY - NUMBER 30

\title{
A Revision of \\ American Velloziaceae
}

\author{
Lyman B. Smith \\ and Edward S. Ayensu
}
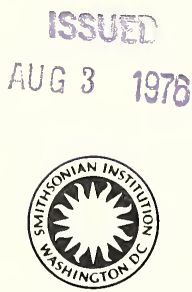

SMITHSONIAN INSTITUTION PRESS

City of Washington

1976 


\section{A B S T RACT}

Smith, Lyman B., and Edward S. Ayensu. A Revision of American Velloziaceae. Smithsonian Contributions to Botany, number 30, 172 pages, frontispiece, 53 figures, 37 plates, 1976.-With the aid of leaf anatomy, the systematics of 4 genera and 229 species of the American Velloziaceae is brought up to date. The sclerenchyma patterns and other anatomical characters that proved diagnostically important in earlier studies, continue to be most useful in delimiting the major genera and species in the present study. An introduction summarizing the major problems yet unravelled in this family and the current and prospective means for solving such problems, are discussed. Taxonomic keys, synonyms, and information on species distribution are included in this revision. Descriptions of new species and of higher taxa are also provided.

ofFICIAL PUBLICATION DATE is handstamped in a limited number of initial copies and is recorded in the Institution's annual report, Smithsonian Year. SERIES COVER DESIGN: Leaf clearing from the katsura tree Cercidiphyllum japonicum Siebold and Zuccarini.

Library of Congress Cataloging in Publication Data

Smith, Lyman B.

A revision of American Velloziaceae.

(Smithsonian contributions to botany ; no. 30)

Bibliography: p.

Supt. of Docs. no.: SI 1.29:30

1. Velloziaceae 2. Botany-America. I. Ayensu, Edward S., joint author. II. Title. III. Series: Smithsonian Institution. Smithsonian contributions to botany ; no. 30 . QK1.S2747 no. 30 ¡QK495.V4] 581'.08s $\left\lceil 584^{\prime} .29\right\rceil 75-619289$ 


\section{Contents}

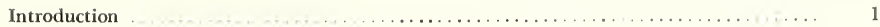

VeLLOZIACEAE

Key to the Subfamilies and Genera of Velloziaceae ......................... 3

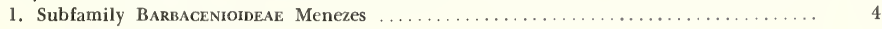

1. Barbacenia Vandelli ........................................ 4

Key to the Subkeys of Barbacenia ............................... 4

Key to the Species of Barbacenia ................................. 4

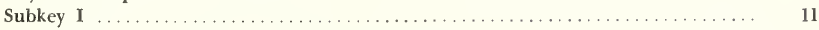

1. Barbacenia brachycalyx Goethart \& Henrard ...................... 11

2. Barbacenia cylindrica L. B. Smith \& Ayensu, new species ............... 11

3. Barbacenia spiralis L. B. Smith \& Ayensu, new species ................ 11

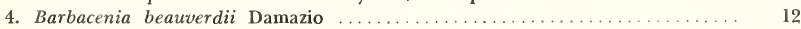

5. Barbacenia pabstiana L. B. Smith \& Ayensu, new species .............. 12

6. Barbacenia caricina Goethart \& Henrard ....................... 13

7. Barbacenia delicatula L. B. Smith \& Ayensu, new species ................ 13

8. Barbacenia squamata Herbert ............................... 13

9. Barbacenia rogieri hortus ex Moore \& Ayres ..................... 13

10. Barbacenia seubertiana Goethart \& Henrard ....................... 13

11. Barbacenia gaveensis Goethart \& Henrard ....................... 14

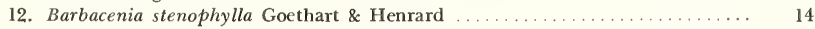

13. Barbacenia inclinata Goethart \& Henrard ........................ 14

14. Barbacenia longiscapa Goethart \& Henrard ....................... 14

15. Barbacenia hatschbachii L. B. Smith \& Ayensu, new species ............. 14

16. Barbacenia monticola L. B. Smith \& Ayensu, new species ............... 14

17. Barbacenia flavida Goethart \& Henrard ........................ 15

18. Barbacenia irwiniana L. B. Smith ........................... 15

19. Barbacenia foliosa Goethart \& Henrard ........................ 15

20. Barbacenia burle-marxii L. B. Smith \& Ayensu, new species ............ 15

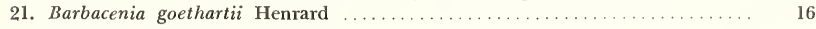

22. Barbacenia brevifolia Taubert ................................ 16

22a. Barbacenia brevifolia var. brevifolia ....................... 16

22b. Barbacenia brevifolia var. recurvata L. B. Smith ................ 16

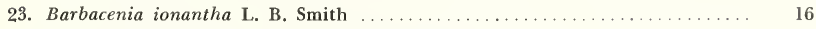

24. Barbacenia mantiqueirae Goethart \& Henrard .................... 16

25. Barbacenia spectabilis L. B. Smith \& Ayensu, new species ................. 17

26. Barbacenia nigrimarginata L. B. Smith ......................... 17

27. Barbacenia gounelleana Beauverd ............................ 17

28. Barbacenia purpurea Hooker ............................... 18

29. Barbacenia andersonii L. B. Smith \& Ayensu, new species ............... 18

30. Barbacenia celiae Maguire .................................... 18

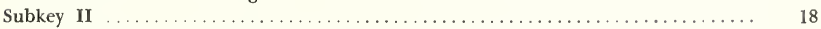

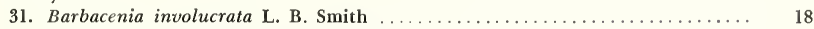

32. Barbacenia schwackei Goethart \& Henrard ........................ 19

33. Barbacenia fulva Goethart \& Henrard .............................. 19

34. Barbacenia polyantha Goethart \& Henrard ....................... 19

35. Barbacenia trigona Goethart \& Henrard ........................ 19

36. Barbacenia globata Goethart \& Henrard ........................ 19

37. Barbacenia coccinea Martius ex Schultes f. ........................ 19

38. Barbacenia glutinosa Goethart \& Henrard ......................... 19

39. Barbacenia markgrafii Schulze-Menz ............................. 19 
40. Barbacenia ignea Martius ex Schultes f.

41. Barbacenia lilacina Goethart \& Henrard

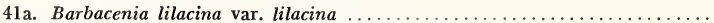

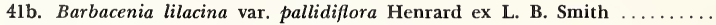

42. Barbacenia vandellii Pohl ex Seubert

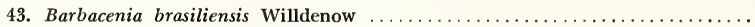

44. Barbacenia nanuzae L. B. Smith \& Ayensu, new species $\ldots \ldots \ldots \ldots \ldots \ldots \ldots$

20

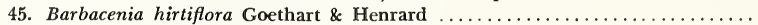

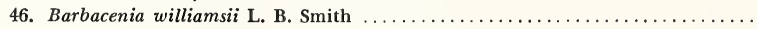

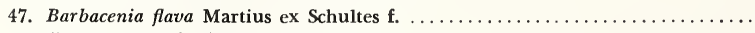

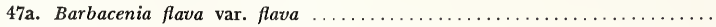

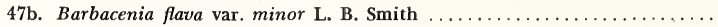

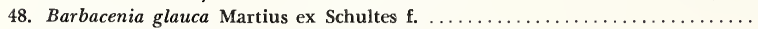

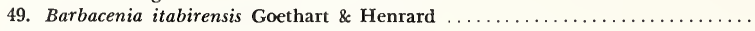

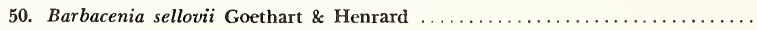
Subkey III

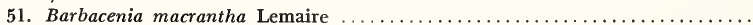

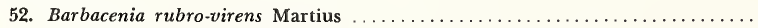

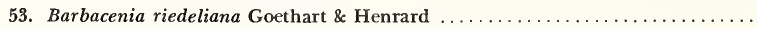

54. Barbacenia chlorantha L. B. Smith \& Ayensu, new species . . . . . . . . . .

55. Barbacenia luzulifolia Martius ex Schultes f. . . . . . . . . . . . . . . .

56. Barbacenia saxicola L. B. Smith \& Ayensu, new species . . . . . . . . . . .

57. Barbacenia reflexa L. B. Smith \& Ayensu, new species $\ldots \ldots \ldots \ldots \ldots \ldots \ldots$

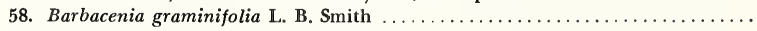

59. Barbacenia latifolia L. B. Smith \& Ayensu, new species $\ldots \ldots \ldots \ldots \ldots \ldots \ldots$

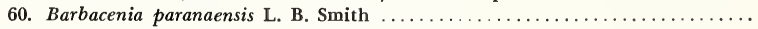

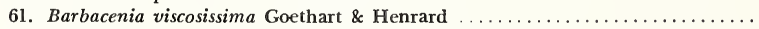

62. Barbacenia umbrosa $\mathbf{L}$. B. Smith \& Ayensu, new species . . . . . . . . . . .

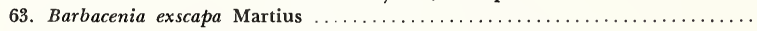

64. Barbacenia rectifolia L. B. Smith \& Ayensu, new species . . . . . . . . . . .

65. Barbacenia aurea $\mathbf{L}$. B. Smith \& Ayensu, new species $\ldots \ldots \ldots \ldots \ldots \ldots \ldots$

66. Barbacenia coronata P. F. Ravenna, new species $\ldots \ldots \ldots \ldots \ldots \ldots \ldots \ldots$

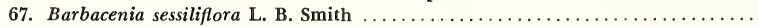

68. Barbacenia nana L. B. Smith \& Ayensu, new species $\ldots \ldots \ldots \ldots \ldots \ldots \ldots \ldots$

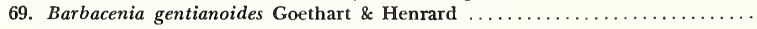

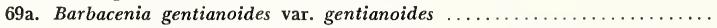
69b. Barbacenia gentianoides var. magalhaesii (L. B. Smith) L. B. Smith \&

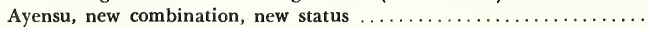

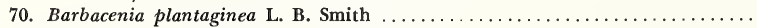

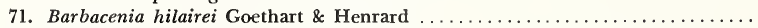

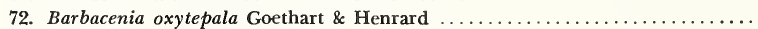

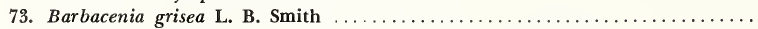

74. Barbacenia filamentifera L. B. Smith \& Ayensu, new species . . . . . . . . .

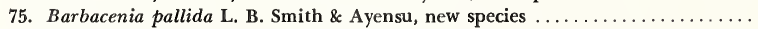

76. Barbacenia salmonea L. B. Smith \& Ayensu, new species $\ldots \ldots \ldots \ldots \ldots \ldots$

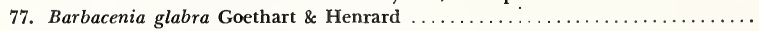

78. Barbacenia nuda L B. Smith \& Ayensu, new species $\ldots \ldots \ldots \ldots \ldots \ldots \ldots \ldots$

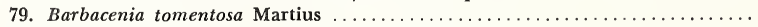

80. Barbacenia cyananthera L. B. Smith \& Ayensu, new species . . . . . . . . . .

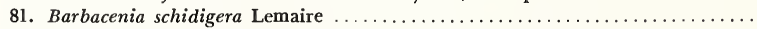

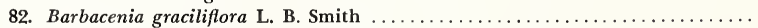

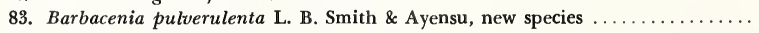

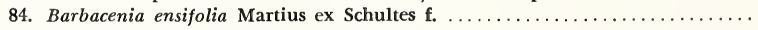

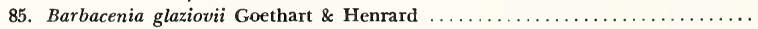

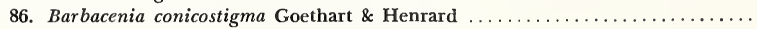

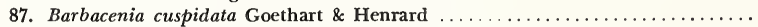

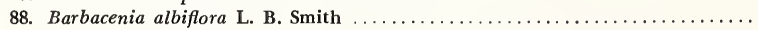

89. Barbacenia bibiriensis L. B. Smith \&c Ayensu, new species . . . . . . . . . . 


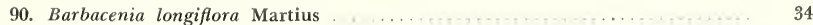

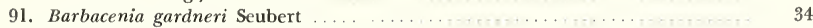

92. Barbacenia curviflora Goethart \& Henrard .. $\ldots \ldots \ldots \ldots \ldots \ldots \ldots \ldots \ldots \ldots$

93. Barbacenia fragrans Goethart \& Henrard . . . . . . . . . . . . . . . . 34

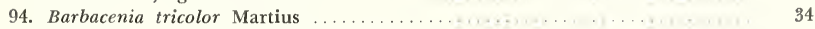

95. Barbacenia blanchetii Goethart \& Henrard . _... . . . . . . . . . . . 35

96. Barbacenia mollis Goethart \& Henrard . . . . . . . . . . . . . . . . . 35

96a. Barbacenia mollis var. mollis . ......................... 35

96b. Barbacenia mollis var. microphylla L. B. Smith _... . 35

97. Barbacenia culta L. B. Smith \& Ayensu, new species . . . . . . . . . 35

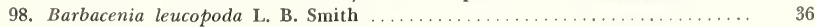

99. Barbacenia sordida $\mathbf{L}$. B. Smith \& Ayensu, new species $\ldots \ldots \ldots \ldots \ldots \ldots \ldots$

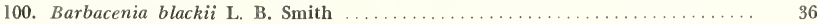

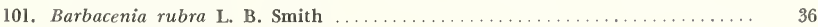

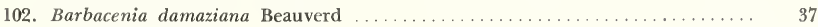

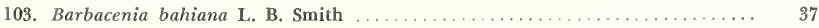

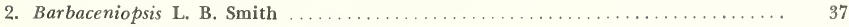

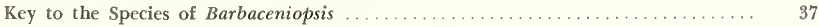

1. Barbaceniopsis boliviensis (Baker) L. B. Smith $\ldots \ldots \ldots \ldots \ldots \ldots \ldots \ldots \ldots$

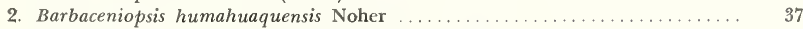

3. Barbaceniopsis vargasiana $($ L. B. Smith) L. B. Smith $\ldots \ldots \ldots \ldots \ldots \ldots \ldots \ldots$

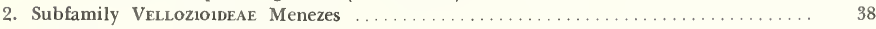

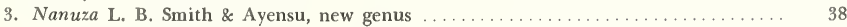

1. Nanuza plicata (Martius) L. B. Smith \& Ayensu, new combination ......... 38

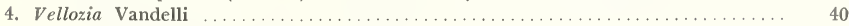

Key to the Sections and Subkeys of Vellozia $\ldots \ldots \ldots \ldots \ldots \ldots \ldots \ldots \ldots \ldots \ldots \ldots \ldots$

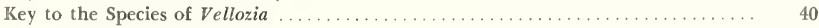

1. Section Xerophytoides L. B. Smith \& Ayensu, new section . . . . . . . . . 48

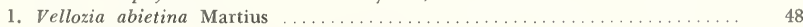

2. Vellozia tragacantha (Martius ex Schultes f.) Martius ex Seubert .......... 48

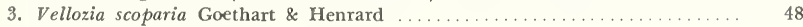

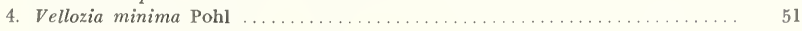

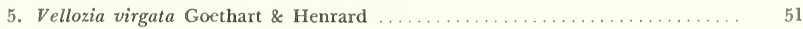

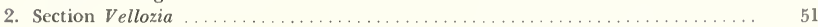

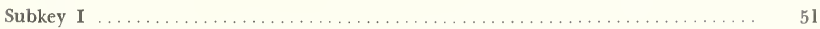

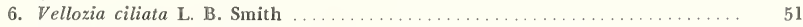

7. Vellozia taxifolia (Martius ex Schultes f.) Martius ex Seubert ........... 51

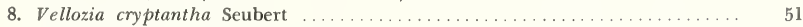

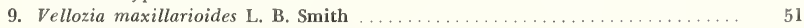

10. Vellozia costata L. B. Smith \& Ayensu, new species $\ldots \ldots \ldots \ldots \ldots \ldots \ldots \ldots$

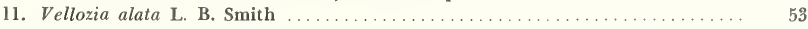

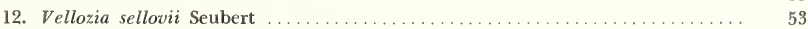

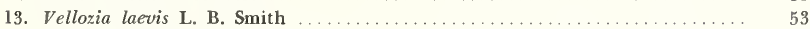

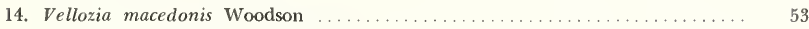

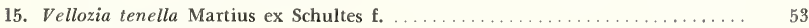

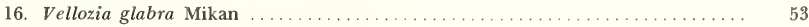

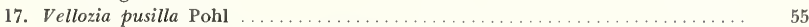

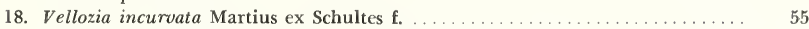

19. Vellozia brachypoda L. B. Smith \& Ayensu, new species . . . . . . . . . 55

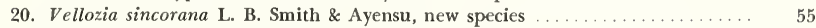

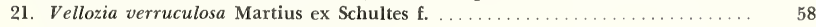

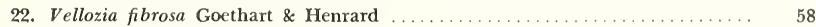

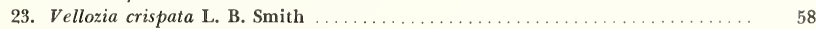

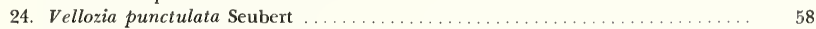

25. Vellozia hatschbachii L. B. Smith \& Ayensu, new species . . . . . . . . . 58

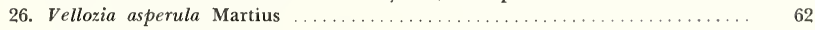

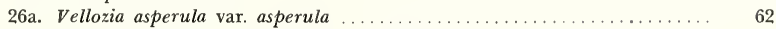

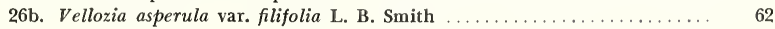


27. Vellozia bradei Schulze-Menz ex Markgraf $\ldots \ldots \ldots \ldots \ldots \ldots \ldots \ldots \ldots$

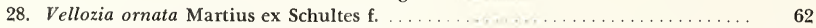

29. Vellozia granulata Goethart \& Henrard . . . . $\ldots \ldots \ldots \ldots \ldots \ldots \ldots \ldots \ldots \ldots \ldots$

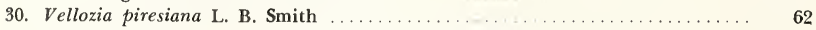

31. Vellozia castanea L. B. Smith \& Ayensu, new species $\ldots \ldots \ldots \ldots \ldots \ldots \ldots$

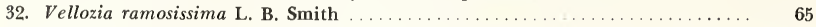

33. Vellozia bicarinata L. B. Smith \& Ayensu, new species $\ldots \ldots \ldots \ldots \ldots \ldots \ldots$

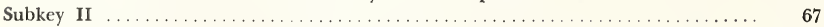

34. Vellozia pterocarpa L. B. Smith \& Ayensu, new species $\ldots \ldots \ldots \ldots \ldots \ldots \ldots$

35. Vellozia angustifolia Goethart \& Henrard $\ldots \ldots \ldots \ldots \ldots \ldots \ldots \ldots \ldots \ldots \ldots$

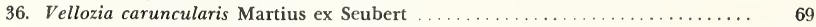

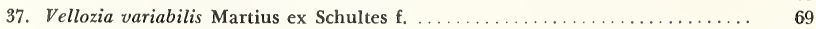

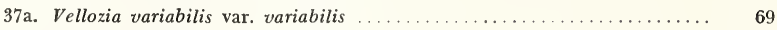

37b. Vellozia variabilis var. tuberculata Seubert $\ldots \ldots \ldots \ldots \ldots \ldots \ldots \ldots \ldots .69$

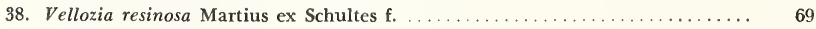

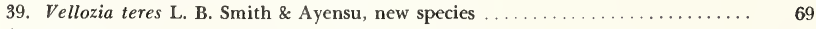

40. Vellozia torquata L. B. Smith \& Ayensu, new species . . . . . . . . . . . 72

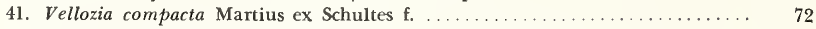

42. Vellozia wasshausenii L. B. Smith \& Ayensu, new species $\ldots \ldots \ldots \ldots \ldots \ldots . \ldots 75$

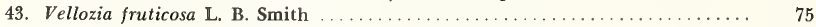

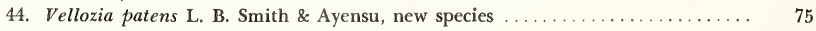

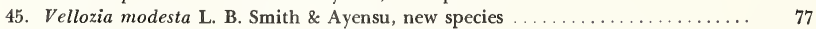

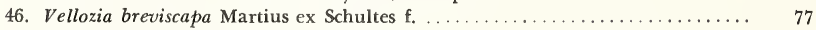

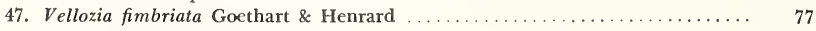

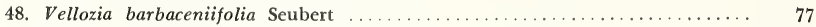

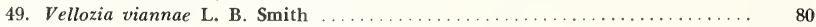

50. Vellozia subalata L. B. Smith \& Ayensu, new species . . . . . . . . . . 80

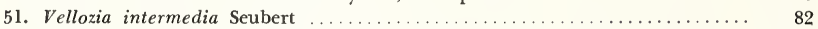

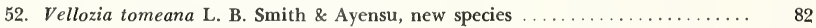

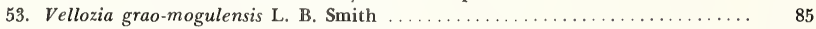

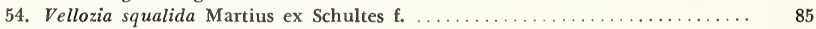

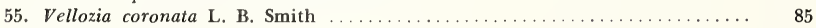

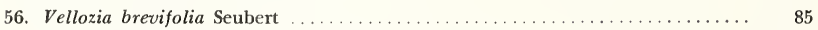

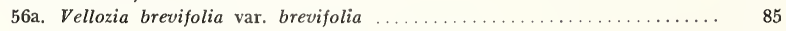

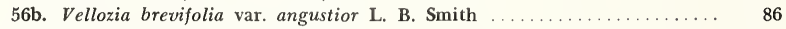

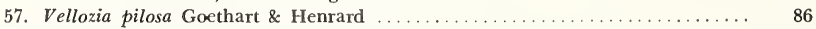

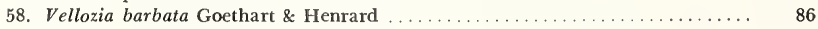

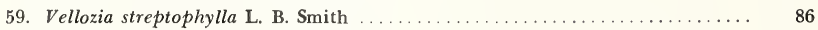

60. Vellozia declinans Goethart \& Henrard $\ldots \ldots \ldots \ldots \ldots \ldots \ldots \ldots \ldots \ldots \ldots$

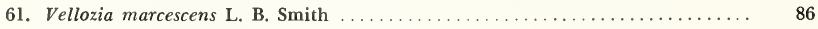

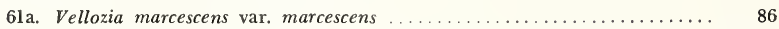

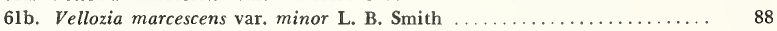

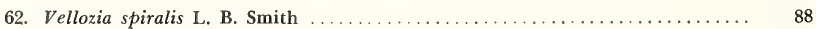

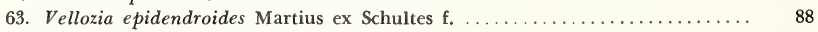

64. Vellozia leptopetala Goethart \& Henrard $\ldots \ldots \ldots \ldots \ldots \ldots \ldots \ldots \ldots \ldots \ldots$

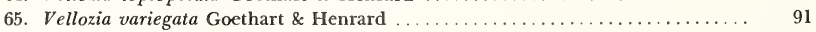

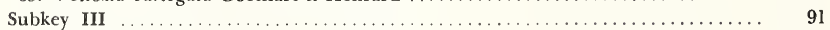

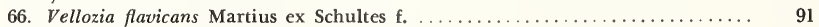

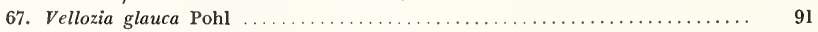

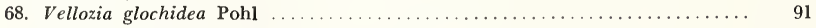

69. Vellozia nuda L. B. Smith \& Ayensu, new species $\ldots \ldots \ldots \ldots \ldots \ldots \ldots \ldots . . \ldots 1$

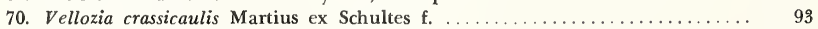

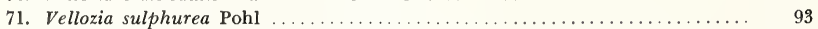

72. Vellozia nivea L. B. Smith \& Ayensu, new species $\ldots \ldots \ldots \ldots \ldots \ldots \ldots \ldots . . \ldots 5$

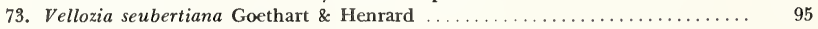

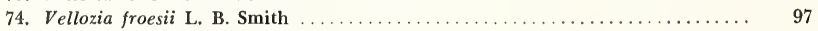

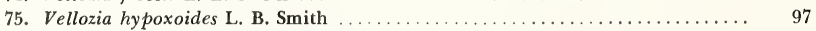

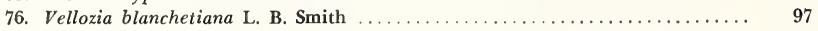


77. Vellozia bulbosa L. B. Smith

78. Vellozia crinita Goethart \& Henrard

79. Vellozia swallenii L. B. Smith

80. Vellozia pumila Goethart \& Henrard

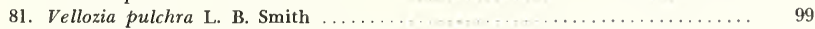

82. Vellozia bahiana L. B. Smith \& Ayensu, new species ................ 99

83. Vellozia cinerascens (Martius ex Schultes f.) Martius ex Seubert ......... 103

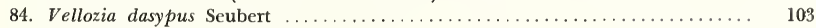

85. Vellozia scabrosa L. B. Smith \& Ayensu, new species $\ldots \ldots \ldots \ldots \ldots \ldots \ldots \ldots$

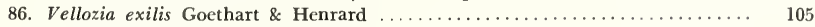

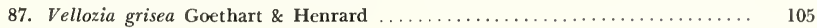

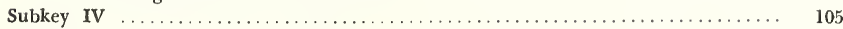

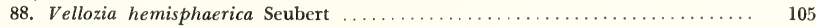

89. Vellozia burle-marxii L. B. Smith \& Ayensu, new species . . . . . . . . . 105

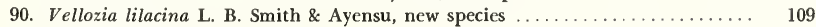

91. Vellozia arenicola L. B. Smith $\ldots \ldots \ldots \ldots \ldots \ldots \ldots \ldots \ldots \ldots \ldots \ldots \ldots \ldots$

92. Vellozia stipitata L. B. Smith \& Ayensu, new species . . . . . . . . . . 109

93. Vellozia decidua L. B. Smith \& Ayensu, new species ............... 112

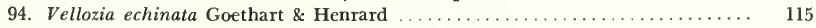

95. Vellozia nanuzae L. B. Smith \& Ayensu, new species $\ldots \ldots \ldots \ldots \ldots \ldots \ldots \ldots$

96. Vellozia geotegens L. B. Smith \& Ayensu, new species $\ldots \ldots \ldots \ldots \ldots \ldots \ldots \ldots$

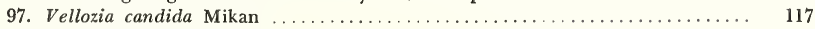

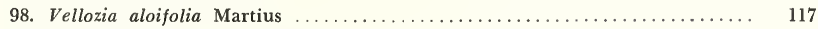

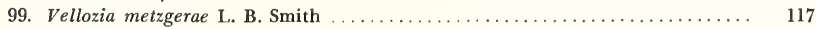

100. Vellozia caespitosa L. B. Smith \& Ayensu, new species ............. 117

101. Vellozia glandulifera Goethart \& Henrard .................... 119

3. Section Radia (A. Richard) L. B. Smith \& Ayensu, new combination, new status. . 119

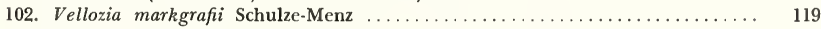

103. Vellozia caput-ardeae L. B. Smith \& Ayensu, new species . . . . . . . . 121

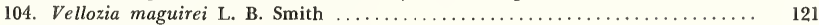

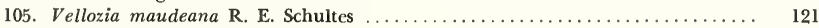

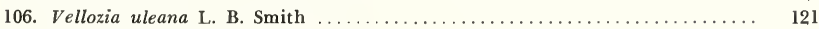

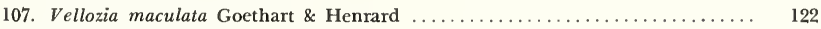

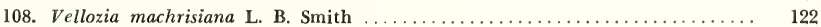

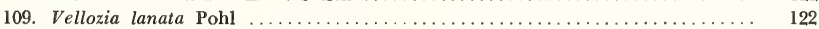

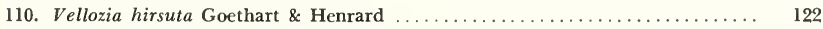

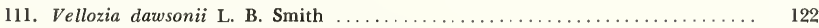

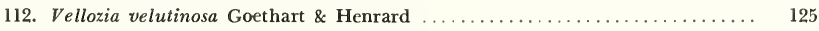

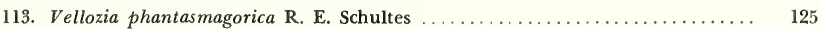

114. Vellozia annulata Goethart \& Henrard $\ldots \ldots \ldots \ldots \ldots \ldots \ldots \ldots \ldots \ldots \ldots \ldots \ldots$

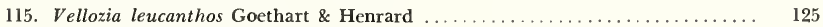

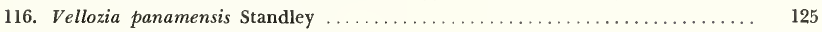

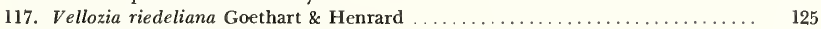

118. Vellozia dumitiana R. E. Schultes $\ldots \ldots \ldots \ldots \ldots \ldots \ldots \ldots \ldots \ldots \ldots \ldots \ldots \ldots \ldots$

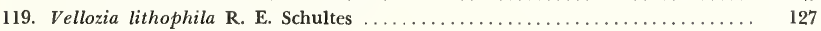

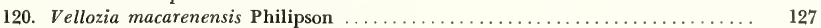

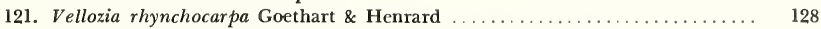

122. Vellozia tubiflora (A. Richard) Humboldt, Bonpland, \& Kunth . . . . . . . 128

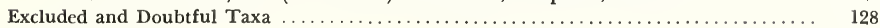

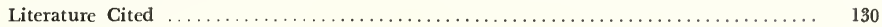

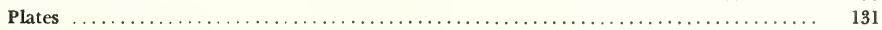

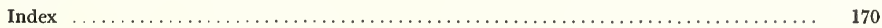




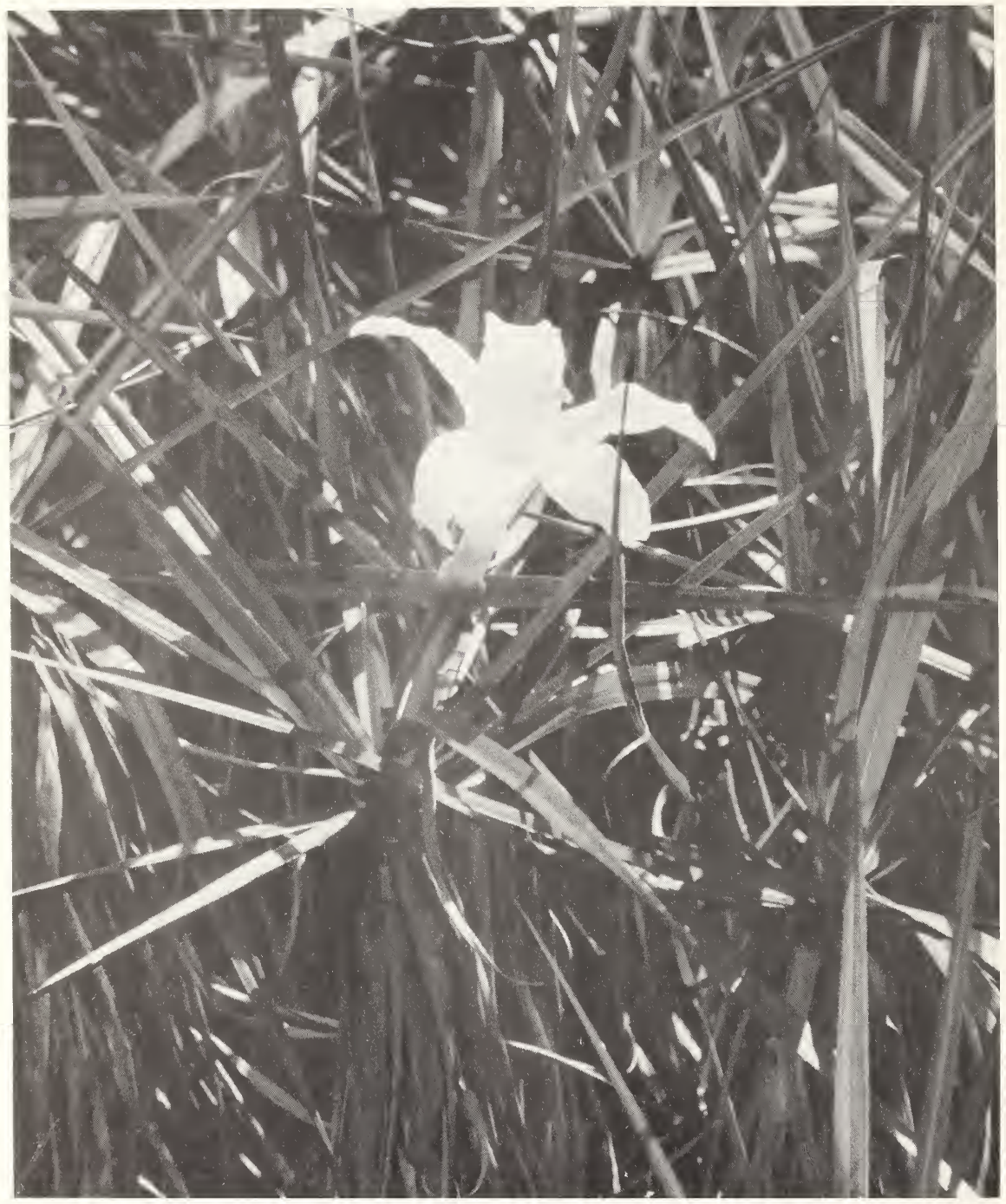

Vellozia hatschbachii L. B. Smith \& Ayensu, new species (photograph by Edward S. Ayensu). 


\title{
A Revision of American Velloziaceae
}

\author{
Lyman B. Smith \\ and Edward S. Ayensu
}

\section{Introduction}

Since Smith's first revision of American Velloziaceae (1962), several times as many collections have become available for study and new techniques of research have been applied to the family. In fact much as we would like to take the time for a monograph it seems advisable for us to take stock of the present situation and expose our findings for use and criticism. On the one hand a number of new species must be published to give a taxonomic base for other lines of research as 28 new species in 103 of Barbacenia and 26 in 122 Vellozia testify. On the other hand generic and subgeneric lines are still unsettled and it is desirable to reach a better accord before proceeding to a monograph.

Collections by the staffs of the United States National Museum, the New York Botanical Garden, and the major Brazilian institutions have greatly increased our knowledge of the American Velloziaceae by numerous new species while showing the extent of variation and the reliability of characters in various old species. At the same time loans from several European herbaria have made possible exact classification and illustration of species that were formerly not well understood.

At present our basic difficulty in the delimitation of genera is the difference between Vellozia and the typically Old World genus Xerophyta. Menezes (1971b) would place certain Brazilian species of Velloziaceae in Xerophyta because they have only 6

Lyman B. Smith and Edward S. Ayensu, Department of Botany, National Museum of Natural History, Smithsonian Institution, Washington, D.C. 20560. stamens, but she would disregard the character of stigma form and type of floral appendages. This stamen character is untenable on two counts, the number 6 or one to each tepal breaks down in certain species with more than 6 but less than 12, while the coronoid appendage character is contradicted. By retaining these species in Vellozia we believe that we have a much more logical system.

Our studies in Barbacenia have progressed less than elsewhere in the family and our arrangement of species here is completely artificial. Menezes has separated one genus, Aylthonia, from Barbacenia (1971b) and has announced but not legally described another at this writing. The single character of connation of the coronoid appendages and the close resemblance of species of Aylthonia to some remaining in Barbacenia make us suspect the new genus of polyphylletic origin and we hesitate to accept it even as a section until we can find some correlating characters to support it. The character still remains a very good one to use in any type of key and it is good to have it brought to notice.

On the other hand Menezes (197la) and Noher and Cocucci (1971:268) are quite correct in their floral anatomical studies of Barbacenia, indicating that what Smith $(1962: 253,271)$ considered a simple filament much dilated is a coronoid appendage to which the filament is fused. In fact this is macro scopically apparent in B. squamata Hooker and strikingly so in our new $B$. spiralis where the $32 \mathrm{~mm}$ long filament is completely free from the coronoid appendage. We are still unable to choose between Menezes' theory of a perianth origin of the coronoid appendages and Noher's of a staminoid. To prove 
their contentions, both cite dissolving technique producing continuous vascular traces yet neither publishes photographic evidence thereof. Perhaps it is as Carlquist (1969) states a matter of no great certainty. In Vellozia the appendages when present are always ventral to the stamens so it would seem reasonable to assume that they are staminal in origin until their structure can be verified.

Leaf-blade anatomy is our most promising source of characters for correlation with the usual macroscopic system because of the availability of material, relative rapidity of technique, and variability among species, especially in Vellozia. Already we have been able to put it to use in defining a new section and redefining an old one in that genus and it is evident that further research will show more groupings of species. In our present keys we have used leaf-blade anatomy to show groupings of species or differences as opportunity offers. Even if complete couplets are unavailable now, there is a good chance of completing them later.

So far the piece of leaf research awaiting exploitation is sheath anatomy. The morphology and anatomy of the leaf sheath in this family, so far as we are aware, is not quite understood. Preliminary examination of this tissue (Ayensu, 1968) has shown that some species exhibit a clear line of abscission between the leaf-blade and the sheathing, but other species do not show this character. Furthermore, the fiber of the sheath is divided into those with tightly parallel fibers with very little reticulate venation and those with few parallel fibers and highly pronounced reticulate venation.

The stem anatomy of some species of the family has been studied in recent years by Ayensu (1968) and Menezes (197la) and the general observations seem to correlate with the major character-states used in distinguishing Vellozia and Barbacenia sensu lato. Not many differences occur in Velloziaceae stems to be used in identifying specific species.

The root anatomy of species of Barbaceniopsis has also been investigated by Ayensu (1968), but again not many specific characters could be assembled to identify individual species.

The pollen morphology (using light, SEM and TEM techniques) has received much attention in recent years by Maguire (1969), Ayensu (1972, 1973), and Ayensu and Skvarla (1974). Essentially these studies show that the pollen morphology of Vellozia (united tetragonal tetrads) is strikingly different from that of Barbacenia (monosulcate monads). Furthermore, inasmuch as some Vellozia species lend themselves to easy specific identification, Barbacenia species, on the other hand, are not easily identified at the specific level.

It is hoped that future chromosomal studies will shed further light in our attempt to clearly define the species.

We give thanks to the directors of the following herbaria for the privilege of examining their specimens or photographs thereof:

BA Museo Argentino de Ciencias Naturales "Bernardino Rivadavia," Buenos Aires

BM British Museum (Natural History), London

F Field Museum of Natural History, Chicago

GH Gray Herbarium of Harvard University, Cambridge, Mass.

IAN Instituto de Pesquisas e Experimentação Agropecuaria do Norte, Belém

K Royal Botanic Gardens, Kew

L Rijksherbarium, Leiden

LE V. L. Komaroff Botanical Institute of the Academy of Sciences of the U.S.S.R., Leningrad

M Botanische Staatssammlung, Munich

MBM Museu Botanico Municipal, Curitiba

MG Museu Paraense Emilio Goeldi, Belém

NY New York Botanical Garden

P Muséum National d'Histoire Naturelle, Paris

R Museu Nacional, Rio de Janeiro

RB Jardim Botânico do Rio de Janeiro

S Naturhistoriska riksmuseet, Stockholm

SGO Museo Nacional de Historia Natural, Santiago

SP Instituto de Botânica, São Paulo

SPF Instituto de Biosciencias, Universidade de São Paulo

UB Universidade de Brasilia

UC University of California, Berkeley

We are most grateful to Ms. Cynthia Bailey for her assistance in the preparation of this revision. Our thanks is also extended to Mr. Eduardo Barros for the preparation of many of the anatomical slides used in this work and to Ms. Alice R. Tangerini for the line drawing illustrations.

\section{VELLOZIACEAE}

Velloziaceae Endlicher, Enchiridion 101, 1841 ['Vellozieae']. -Seubert in Martius, Fl. Bras., 3(1):65, 1847 ['Vellozieae']. -Drude in Schenck, Handb. Bot., 333, 1886.-Pax in Engler \& Prantl, Pflanzenfam., 2, Abt. 5:125, 1887; ed. 2, 15a:431, 1930.-Hutchinson, Fam. Fl. Pl. ed. 3, [834], fig. 407, 1973.

Amaryllideae-Vellozieae D. Don, Edinb. New Philos. Journ., 8:164, 1830.-Baker in Bentham \& Hooker, Gen. Pl. 3:739, 1880 . 
Perennial more or less shrubby plants; indument highly diverse, often not strictly epidermal (Greves, 1921:274). Caudex (stem) woody, fibrous, simple or dichotomously branched, covered with persistent leaves or at least by their imbricate sheaths. Leaves clustered at the end of the stem or its branches when new, the grass-like blades then reflexing or deciduous by a regular usually straight transverse line.

Scapes one or more, deciduous in age or becoming lateral by the elongation of the axis. Flowers solitary on each scape, perfect or rarely functionally unisexual (Barbaceniopsis), actinomorphic, usually brightly colored. Perianth-tube equaling to greatly exceeding the ovary and adnate to it, often bearing 6 separate or united coronoid appendages dorsal to the stamens and usually united to them. Tepals 6 in 2 series but mostly very similar, erect to reflexed at anthesis. Stamens 6 to numerous in 6 bundles or phalanges; filaments free, terete and simple and often bearing basal appendages ventrally (Vellozia) or often fusing with the coronoid appendages (Barbacenia, Xerophyta) or with the tepals (Barbaceniopsis); anthers basifixed to medifixed, opening by longitudinal slits, entire or divided at base. Ovary 3-locular, inferior, often depressed at apex or the walls prolonged upward into an epigynous tube; style slender but usually enlarged by the stigmas; stigmas 3, apical or subapical, distinct to wholly confluent, linear to orbicular; ovules numerous in many rows on stalked placentas. Fruit a capsule; seeds numerous; embryo small; endosperm copious, nonfarinaceous.

Leaf ANatomy.-Trichomes present, mostly hairs but no scales. Leaf-blade dorsiventral, V-shaped with distinct median vein (sometimes inconspicuous in some species of Vellozia). Epidermal walls straight, somewhat rectangular. Cuticle thin but slightly thicker around stomata and on abaxial epidermis. Stomata occurring on both epidermal surfaces, but principally on abaxial surface, their ledges mostly inconspicuous; subsidiary cells mostly paracytic, some tetracytic. Hypodermis if present not distinct. Mesophyll consisting of palisade and spongy tissue. Vascular bundles of leaf collateral, fairly equal sizes, each associated with a pair of distinctive sclerenchyma girders, varying in number from species to species. Phloem units usually 2 in mature leaves, lying along the arms of the abaxial $U$ - or $Y$-shaped girder. Bundle sheath extending to the abaxial and in some genera to the adaxial epidermis as well, central and marginal bundles associated with sclerenchyma. Commissural bundles often present. Crystal present mostly raphide bundles; tannin present in many species.

Type-Genus._Vellozia Vandelli.

\section{Key to the Subfamilies and Genera of Velloziaceae \\ (including Old World)}

1. Stigmas vertical or nearly so, apical or sometimes subapical, separate to completely confluent, elliptic to linear; stamens always 6 , the filaments usually not evident or appearing broadly flattened; appendages when present dorsal to the stamens; pollen grains single; adaxial epidermis in contact with the bundle sheath or not ........ Subfamily BARBACENIOIDEAE 2. Stamens adjacent to the style, the filaments free from the tepals and without any type of appendages, cylindric, the anthers lateral to extrorse; xylem in leaf composed mainly of tracheids; leaf-blades persistent, mesophytic. South Africa ............... Talbotia

2. Stamens adjacent or adnate to the tepals, the filaments appearing much flattened from fusion with a coronoid appendage or rarely cylindric with the appendage between them and the tepals (Barbacenia spiralis) or fused to the tepals and without appendages, the anthers introrse; xylem in leaf composed mainly or vessels; leaf-blades persistent to deciduous, submesophytic to xerophytic.

3. Flowers perfect; coronoid appendages present between the filaments and the tepals and usually fused with the filaments making them appear broad and flat.

4. Coronoid appendages ("filaments") largely adnate to the bases of the tepals; leafblades usually deciduous. Africa, Madagascar, Arabia ........... Xerophyta

4. Coronoid appendages wholly or almost wholly free from the tepals; leaf-blades persistent; epidermis in contact with bundle sheaths on both sides. Brazil

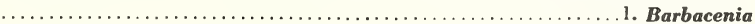

3. Flowers functionally unisexual; plants dioecious; coronoid appendages lacking and filaments highly fused with tepals so that anthers appear sessile; leaf-blades persistent; sides about equally furrowed. Peru, Bolivia, Argentina 2. Barbaceniopsis 
1. Stigmas horizontal to somewhat reflexed, always apical and apically confluent, suborbicular; stamens 6 to numerous, the filaments evident, slenderly cylindric; appendages when present always ventral to the stamens; pollen grains tetraploid or single; adaxial epidermis not in

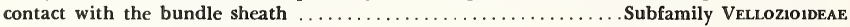

5. Caudex with leaf-sheaths strongly trigonous; leaf-blades deciduous, strongly plicate, the adaxial side more deeply furrowed than the abaxial; stamens only 6; pollen in single

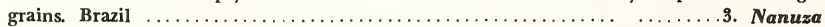

5. Caudex with leaf-sheaths terete; leaf-blades persistent or deciduous, not plicate, even or nearly so on the adaxial side; stamens usually more than 6; pollen in tetrads. Panama, South America 4. Vellozia

\section{Subfamily BARBACENIOIDEAE Menezes}

Barbacenioideae Menezes, Ciência e Cultura, 23(3):[421], 1971 [emend. L. B. Smith \& Ayensu].

Stigmata verticalia vel subverticalia haud suborbicularia.

Floral appendages when present dorsal to the stamens. Stamens always 6; filaments usually not evident or appearing broad and flattened, rarely free and cylindrical but then associated with a floral appendage; anthers typically dorsifixed; pollen grains single. Stigmas vertical or nearly so, apical or sometimes subapical, separate to completely confluent, elliptic to linear.

Adaxial epidermis in contact with the bundle sheath or not.

Note: Original description emended to exclude species with 6 stamens but with horizontal stigmas, as well as the characters cited by Menezes.

\section{Barbacenia Vandelli}

Barbacenia Vandelli, Fl. Lusit. \& Brasil. Spec., 21, pl. 1, 1788.
Pleurostima Rafinesque, Fl. Tellur., 2:97, 1836 [type: Barbacenia purpurea Hooker].

Visnea Steudel ex Endlicher, Gen., 173, 1837 [nomen in synonymy].

Veitchia Herbert, Bot. Reg. 29, Misc.: 64, 1843 [nomen in synonymy].

Aylthonia Menezes, Ciência e Cultura, 23(3):[421], 1971 [type: Barbacenia gentianoides Goethart \& Henrard.]

Plants of small or medium stature. Caudex usually simple or few-branched. Leaf-blades longpersistent.

Flowers perfect. Perianth forming more or less of a tube above the ovary; coronoid appendages free from the tepals but usually adnate to the filaments and making them appear broad and flat, usually bilobed. Stamens 6; filaments usually evident but anthers rarely subsessile (Barbacenia squamata); anthers dorsifixed. Stigmas inconspicuous, vertical or nearly so.

LeAF ANATOMY.-Bundle sheaths in contact with the epidermis on both sides. Furrows mostly lacking.

TYPE-SPECIEs.-Barbacenia brasiliensis Willdenow.

\section{Key to the Subkeys of Barbacenia}

1. Perinath-tube (including section fused to ovary) not more than twice as long as the ovary. 2. Ovary section of the perianth partly or wholly glabrous ................ Subkey I

2. Ovary section of the perianth wholly vestite $\ldots \ldots \ldots \ldots \ldots \ldots \ldots \ldots \ldots$ Subkey II

1. Perianth-tube more than twice as long as the ovary, i.e., the free part longer than the part fused to the ovary Subkey III

\section{Key to the Species of Barbacenia}

\section{SUBKEY I}

1. Perianth-tube completely glabrous or with a few trichomes at extreme base.

2. Stem (including leaf-bases) $13-15 \mathrm{~mm}$ thick; leaves more than 3-ranked, their sheaths almost or completely covered; blades to $14 \mathrm{~mm}$ wide; flowers red.

3. Tepals to $25 \mathrm{~mm}$ long; perianth-tube cylindric or subcylindric; filaments adnate to broad coronoid appendages; old leaf-blades nearly straight. Brazil: Goiás. 
4. Perianth-tube $10 \mathrm{~mm}$ long, less than half the tepals

1. B. brachycalyx

4. Perianth-tube $30 \mathrm{~mm}$ long, longer than the tepals; leaf: adaxial sclerenchyma caps contacting vascular bundles ............... . cylindrica, new species

3. Tepals $50 \mathrm{~mm}$ long; perianth-tube broadly obconic; filaments free, cylindric; old leafblades recurved in a tight spiral; adaxial sclerenchyma caps contacting vascular bundles. Brazil: Minas Gerais

3. B. spiralis, new species

2. Stem (including leaf-bases) slender; leaves obviously 3-ranked, their sheaths much exposed; blades $2.5-8 \mathrm{~mm}$ wide.

5. Flowers 10-13 mm long, violet; leaves about twice peduncles. Brazil: Minas Gerais

5. Flowers $20-30 \mathrm{~mm}$ long.

6. Flowers violet or blue, $30 \mathrm{~mm}$ long; leaf: adaxial sclerenchyma caps contacting vascular bundles. Brazil: Espírito Santo ..........5. B. pabstiana, new species

6. Flowers red or green.

7. Capsule $15 \mathrm{~mm}$ long; flowers red. Brazil: Rio de Janeiro .......6. B. caricina

7. Capsule $7 \mathrm{~mm}$ long; leaf: adaxial sclerenchyma caps separated from vascular bundles; flowers green. Brazil: Minas Gerais .....7. B. delicatula, new species

1. Perianth-tube vestite on the costae or laxly and generally.

8. Indument of the perianth of pointed trichomes, eglandular.

9. Anthers twice as long as the filaments; filaments free. Brazil: Rio de Janeiro (city and state)

8. B. squamata

9. Anthers exceeded by the coronoid appendages; filaments adnate.

10. Tepals $40 \mathrm{~mm}$ long, ample; leaf-blades $9 \mathrm{~mm}$ wide. Brazil: Rio de Janeiro

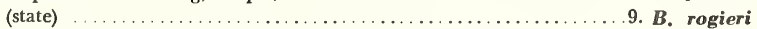

10. Tepals $20-30 \mathrm{~mm}$ long, narrow; leaf: indistinct palisade-like cells present.

11. Leaf-blades $7-9 \mathrm{~mm}$ wide. Brazil: Rio de Janeiro (city and state)

10. B. seubertiana

11. Leaf-blades $5 \mathrm{~mm}$ wide. Brazil: Rio de Janeiro (city) . . . . 11. B. gaveensis

8. Indument of the perianth of sessile (verruculose) or stipitate glands.

12. Anthers almost equaling or exceeding the coronoid appendages.

13. Leaf-blades $2 \mathrm{~mm}$ wide; palisade cells lacking; perianth-tube $8 \mathrm{~mm}$ long. Brazil: Goiás

12. B. stenophylla

13. Leaf-blades 4-18 $\mathrm{mm}$ wide; perianth-tube $13-20 \mathrm{~mm}$ long.

14. Perianth-tube about twice as long as the ovary; leaf-blades $12-18 \mathrm{~mm}$ wide.

Brazil: Minas Gerais

13. B. inclinata

14. Perianth-tube not more than half again as long as the ovary.

15. Scape $20-35 \mathrm{~cm}$ long.

16. Leaf-blades $12-18 \mathrm{~mm}$ wide; palisade cells present. Brazil: Minas Gerais

14. B. longiscapa

16. Leaf-blades 4-10 $\mathrm{mm}$ wide.

17. Tepals $35 \mathrm{~mm}$ long; leaf: palisade cells lacking. Brazil: Mato Grosso ................. 15. B. hatschbachii, new species

17. Tepals $10 \mathrm{~mm}$ long; leaf: palisade-like cells present. Brazil: Minas Gerais ............... B. monticola, new species

15. Scape ca. $15 \mathrm{~cm}$ long.

18. Leaf-blades $9-11 \mathrm{~mm}$ wide; filaments $8 \mathrm{~mm}$ long. Brazil: Minas

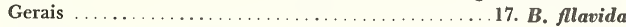

18. Leaf-blades 4-8 $\mathrm{mm}$ wide.

19. Tepals glandular-punctate; costae of the ovary wholly covered by black stipitate glands; leaf: palisade cells present. Brazil: Minas Gerais

18. B. irvoiniana

19. Tepals glabrous; costae laxly glandular or the intermediate ones glabrous.

20. Filaments free, ca. $4 \mathrm{~mm}$ long, about half as long as the anthers; leaves clustered at apex of stem; (perianth-tube described as glandular, but not so depicted). Rio de Janeiro (state) $\ldots \ldots \ldots \ldots \ldots \ldots \ldots \ldots \ldots \ldots . . .6$. squamata

20. Filaments adnate, the appendages $7-8 \mathrm{~mm}$ long, slightly shorter than the anthers if at all. 
21. Tepals acute to attenuate; leaf-blades $8 \mathrm{~mm}$ wide. Rio de Janeiro (city) 19. B. foliosa

21. Tepals rounded and apiculate; leaf-blades $4 \mathrm{~mm}$ wide; palisade-like cells present. Brazil: Espírito Santo .... $\ldots \ldots \ldots \ldots \ldots \ldots$ 20. B. burle-marxii, new species

12. Anthers exceeded by the coronoid appendages.

22. Scape $1 \mathrm{~cm}$ long; perianth-tube about twice as long as the ovary and forming a slender tube above it; anthers medifixed. Brazil: Minas Gerais . 21. B. goethartii

22. Scape elongate; perianth-tube much less than twice as long as the ovary.

23. Leaf-blades $6-8 \mathrm{~cm}$ long, 2-8 $\mathrm{mm}$ wide; anthers equaling or exceeding the base of the filament-sinus. Brazil: Minas Gerais.

24. Scapes 2-3 times the leaves. Brazil. São Paulo ......... 22. B. brevifolia

24. Scapes about equaling the leaves. Brazil: Minas Gerais . 23. B. ionantha

23. Leaf-blades nearly twice as large or more; mostly more than half as long as the scape.

25. Leaf-blades entire; costae stipitate-glandular; style subulate, scarcely dilated by the stigmas. Brazil: São Paulo .......... 24. B. mantiqueirae

25. Leaf-blades denticulate or spinulose-serrate.

26. Tepals 40-50 mm long, ample; leaf-blades 9-15 mm wide.

27. Trichomes sparse and limited to the costae (perianth-tube described as glandular, but not so depicted); tepals $40 \mathrm{~mm}$ long. Brazil: Rio de Janeiro (state) ............. B. rogieri

27. Trichomes numerous and general on the perianth-tube; tepals $50 \mathrm{~mm}$ long; leaf: palisade cells present. Brazil: Espírito Santo $\ldots \ldots \ldots \ldots \ldots \ldots \ldots \ldots \ldots \ldots \ldots \ldots$. B. spectabilis, new species

26. Tepals 16-30 $\mathrm{mm}$ long, narrow.

28. Leaf-blades with narrow dark margins and keel, denticulate; stigmas apical, making the style slenderly conical. Brazil: Goiás $\ldots \ldots \ldots \ldots \ldots \ldots \ldots \ldots \ldots \ldots \ldots \ldots \ldots \ldots \ldots \ldots \ldots \ldots$

28. Leaf-blades concolorous.

29. Stigmas apical on the style, making it clavate; anthers acuminate exceeding the base of the appendage sinus; leafblades denticulate; both sides undulating. Brazil: Rio de Janeiro (state) ................ B. gounelleana

29. Stigmas subapical or submedian on the style.

30. Tepals attenuate; leaf: both sides strongly furrowed. Brazil: Rio de Janeiro (city) ....... 28. B. purpurea

30. Tepals obtuse; leaf: both sides undulating.

31. Scapes about twice the leaves. Brazil: Goiás ...... $\ldots \ldots \ldots \ldots \ldots$. 29 . andersonii, new species

31. Scapes about equaling the leaves. Brazil: Roraima $\ldots \ldots \ldots \ldots \ldots \ldots \ldots \ldots \ldots \ldots \ldots \ldots \ldots \ldots \ldots$. B. celiae

\section{SUBKEY II}

(Brazil: Minas Gerais)

1. Indument of the perianth wholly of pointed trichomes, not glandular; leaf: no furrows.

2. Flowers each with an involucre of ample bracts; leaf: palisade-like cells present $\ldots \ldots \ldots$

2. Flowers without an involucre.

3. Tepals only about $1 / 3$ as long as the tube; leaf-blades hirsute beneath; palisade-like cells absent, mesophyll undifferentiated ............. schwackei

3. Tepals 1-2 times as long as the tube; leaf-blades glabrous $\ldots \ldots \ldots \ldots \ldots$ 33. B. fulva

1. Indument of the perianth glandular, either sessile or stipitate.

4. Scapes up to 12 per rosette; leaves up to 40 ; sheaths resinous-conglutinated; flowers violet; tepals 4-5 mm long.

4. Scapes 1-5 per rosette.

34. B. polyantha 
5. Leaf-blades uniformly and persistently vestite.

6. Ovary trigonous, verrucose

35. B. trigona

6. Ovary terete or if subtrigonous then covered with stipitate glands.

7. Flowers yellow; perianth-tube $12 \mathrm{~mm}$ long, twice as long as the ovary ....

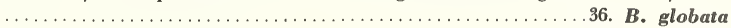

7. Flowers red to lilac, or white.

8. Glands of the perianth-tube sessile; perianth-tube twice as long as the ovary

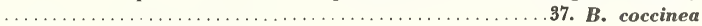

8. Glands of the perianth-tube stipitate.

9. Leaf-indument glandular.

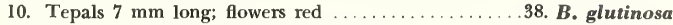

10. Tepals 17-20 mm long; flowers white ......... 39. B. markgrafii

9. Leaf-indument eglandular.

11. Perianth-tube $25 \mathrm{~mm}$ long; flowers red; leaf: no furrows, no palisade cells

40. B. ignea

11. Perinath-tube $20 \mathrm{~mm}$ long; flowers lilac ......... 41. B. lilacina

5. Leaf-blades soon glabrous except for the margins and keel or wholly glabrous.

12. Glands of the ovary sessile; perianth-tube $50 \mathrm{~mm}$ long; anthers medifixed, nearly twice as long as the coronoid appendages; flowers yellow; leaf: both sides strongly undulating, no palisade cells

42. B. vandellii

12. Glands of the ovary stipitate.

13. Anthers not more than equaling the coronoid appendages.

14. Stigmas apical on the style; anthers equaling the appendages

14. Stigmas about $9 \mathrm{~mm}$ below the subulate apex of the style; anthers much shorter than the appendages ............ 44 . nanuzae, new species

13. Anthers exceeding the coronoid appendages.

15. Trichomes of the upper scape long, slender, mostly or entirely eglandular. 16. Flowers deep lilac; leaf-blades $10 \mathrm{~mm}$ wide ......45. B. hirtiflora

16. Flowers yellow; leaf-blades $7 \mathrm{~mm}$ wide; no furrows; no palisade cells $\ldots \ldots \ldots \ldots \ldots \ldots \ldots \ldots \ldots \ldots \ldots \ldots \ldots \ldots \ldots \ldots \ldots \ldots \ldots \ldots \ldots \ldots \ldots \ldots$. williamsii

15. Trichomes of the upper scape short, stout, coarsely glandular; tepals $15-17$ $\mathrm{mm}$ long.

17. Free part of perianth-tube subcylindric; whole tube $15-23 \mathrm{~mm}$ long; leaf: no furrows, no palisade cells . . . . . . . . . . Alava

17. Free part of perianth-tube campanulate; whole tube $25-45 \mathrm{~mm}$ long. 18. Perianth-tube $32-45 \mathrm{~mm}$ long; caudex 3-4 dm high 48 . B. glauca 18. Perianth-tube $25 \mathrm{~mm}$ long; caudex very short.

19. Leaf-blades $40 \mathrm{~cm}$ long, $8-10 \mathrm{~mm}$ wide, glaucous

49. B. itabirensis

19. Leaf-blades $9-16 \mathrm{~cm}$ long, 4-7 $\mathrm{mm}$ wide, dull (?); no furrows, no palisade cells

50. B. sellovii

\section{SUBKEY III}

1. Free part of perianth-tube infundibuliform, distinctly wider at the mouth than at the base. 2. Tepals broadly ovate, as broad as long, perianth-tube $35 \mathrm{~mm}$ long; flowers subsessile; leaf: hairy and undulating on both sides, palisade cells present. Brazil: Minas Gerais

2. Tepals oblong, lanceolate, or elliptic, much longer than broad.

3. Perianth-tube $60-85 \mathrm{~mm}$ long. Brazil: Minas Gerais.

4. Tepals reflexed, $40 \mathrm{~mm}$ long; perianth-indument eglandular, fine, dense; leaf-blades glabrous except for cilia $\ldots \ldots \ldots \ldots \ldots \ldots \ldots \ldots \ldots \ldots \ldots \ldots \ldots \ldots \ldots \ldots$. rubro-virens

4. Tepals erect, $20 \mathrm{~mm}$ long; perianth-indument glandular, very lax.

5. Perianth-tube yellow, $8 \mathrm{~mm}$ wide at apex; leaf-blades hairy on both sides

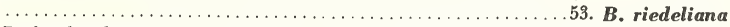

5. Perianth-tube green, $13 \mathrm{~mm}$ wide at apex; leaf-blades ciliate, otherwise glabrous 54. B. chlorantha, new species 
3. Perianth-tube $30-55 \mathrm{~mm}$ long.

6. Indument of perianth filamentous, attenuate, eglandular; perianth-tube $30-45 \mathrm{~mm}$ long; leaf-blades glabrous except for the long-ciliate margins. Brazil: Minas Gerais.

7. Filaments more than half as long as the anthers; coronoid appendages narrow, acute; leaf-blade glabrous, both sides undulating, palisade-like cells present

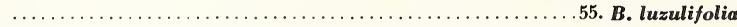

7. Filaments less than half as long as the anthers; leaf-blades glabrous.

8. Tepals suberect; coronoid appendages broadly rounded

56. B. saxicola, new species

8. Tepals reflexed; coronoid appendages acute ...57. B. reflexa, new species

6. Indument of perianth glandular; perianth-tube $40-55 \mathrm{~mm}$ long.

9. Leaf-blades glabrous. Brazil: Minas Gerais ............58. B. graminifolia

9. Leaf-blades evenly vestite all over or at least toward base.

10. Tepals over $30 \mathrm{~mm}$ long; perianth-tube slightly more than twice the ovary; leaf-blades to $23 \mathrm{~mm}$ wide, pubescent and ridged on both sides, no palisade cells. Brazil: Minas Gerais .............. B. latifolia, new species

10. Tepals 15-20 mm long; perianth-tube 3-6 times ovary.

11. Coronoid appendages broadly triangular, wider than high; perianthtube 3 times ovary; leaf-blades to $17 \mathrm{~mm}$ wide, pubescent and ridged on both sides, palisade cells present. Brazil: Paraná .60. B. paranaensis

11. Coronoid appendages rectangular or narrowly triangular, much higher than wide; perianth 4-6 times ovary. Brazil: Minas Gerais.

12. Scapes less than half as long as the leaves; leaves $9 \mathrm{~mm}$ wide, pubescent on both sides; perianth-tube $55 \mathrm{~mm}$ long, 5-6 times the ovary $\ldots \ldots \ldots \ldots \ldots \ldots \ldots \ldots \ldots \ldots \ldots \ldots \ldots \ldots \ldots$. viscosissima

12. Scapes about equaling the leaves; leaves $6 \mathrm{~mm}$ wide, pubescent beneath; perianth-tube $45-50 \mathrm{~mm}$ long

62. B. umbrosa, new species

1. Free part of perianth-tube slenderly cylindric or very slightly enlarged toward apex.

13. Indument of the perianth subulate or lacking, rarely a few fine trichomes slightly enlarged at apex (B. nana). Brazil: Minas Gerais.

14. Scapes none or very short and completely hidden by the leaves.

15. Tepals broadly elliptic, 10-15 mm long; flowers yellow.

16. Leaf-blades arched-recurving, 9-15 $\mathrm{mm}$ wide; long-ciliate; adaxial and abaxial girders poorly developed ................ $63 . \ldots \ldots$

16. Leaf-blades straight, erect to reflexed, $7 \mathrm{~mm}$ wide, laxly and inconspicuously setose-denticulate; adaxial and abaxial girders distinct

64. B. rectifolia, new species

15. Tepals narrow, $12-40 \mathrm{~mm}$ long; flowers red to yellow.

17. Tepals 11-15 mm long; perianth pubescent.

18. Leaf-blades $10 \mathrm{~mm}$ wide, white-pubescent throughout; furrows lacking, palisade cells present $\ldots \ldots \ldots \ldots \ldots \ldots \ldots \ldots 6$. B. aurea, new species

18. Leaf-blades 4-5 mm wide, glabrous, except for the margins.

19. Margins of leaf-blades obscurely denticulate; leaf: furrows lacking palisade cells present $\ldots \ldots \ldots \ldots \ldots \ldots \ldots \ldots \ldots \ldots \ldots \ldots \ldots \ldots \ldots \ldots$

19. Margins of leaf-blades setose-ciliate .......67. B. sessiliflora

17. Tepals $20-40 \mathrm{~mm}$ long; coronoid appendages connate; leaf: furrows lacking, palisade cells present.

20. Perianth subdensely white-pubescent; leaf-blades equally and subdensely white-pubescent throughout $\ldots \ldots \ldots \ldots \ldots 68$. B. nana, new species

20. Perianth glabrous or nearly so; leaf-blades unequally and laxly pubescent if at all $\ldots \ldots \ldots \ldots \ldots \ldots \ldots \ldots \ldots \ldots \ldots \ldots \ldots \ldots \ldots \ldots \ldots \ldots \ldots \ldots \ldots+$ gentianoides

14. Scapes evident, not concealed by the leaves.

21. Perianth-tube $45-60 \mathrm{~mm}$ long.

22. Leaf-blades oblong, 6-9 $\mathrm{cm}$ long, to $20 \mathrm{~mm}$ wide; scape and flower glabrous

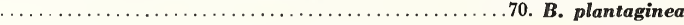

22. Leaf-blades linear, much longer and narrower.

23. Ovary $1 / 5$ of perianth-tube; scape pilose; leaves pubescent $\ldots \ldots \ldots$.

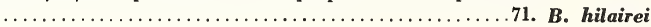


23. Ovary $2 / 5$ of the $45-60 \mathrm{~mm}$ perianth-tube.

24. Scapes glabrous; perianth-tube $50 \mathrm{~mm}$ long, sparsely pilose on the costae; coronoid appendages $10 \mathrm{~mm}$ long; leaf-margin denticulate

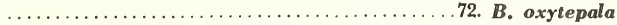

24. Scapes tomentellous toward apex; perianth-tube evenly pilose; leafmargin setose-ciliate toward base.

25. Leaf-blades $13 \mathrm{~mm}$ wide, resinous-punctulate between the nerves; perianth-tube finely and closely gray-tomentellous .... $\ldots \ldots \ldots \ldots \ldots \ldots \ldots \ldots \ldots \ldots \ldots \ldots \ldots \ldots \ldots \ldots \ldots \ldots \ldots \ldots \ldots \ldots$. B. grisea

25. Leaf-blades 5-6 mm wide, ciliate, otherwise glabrous; leaf: furrows lacking, palisade cells present; perianth-tube pilose.

26. Perianth-tube nearly or quite straight, laxly pilose; leafblades evenly attenuate; scape shorter than the leaves.

flower red . . . . . . . . Filamentifera, new species

26. Perianth-tube nearly or quite straight, laxly pilose; leafblades evenly attenuate; scape shorter than the leaves.

27. Coronoid appendages $12 \mathrm{~mm}$ long; stamens erect; flower white .............. pallida, new species

27. Coronoid appendages $3 \mathrm{~mm}$ long; stamens decurved; flower salmon .......76. B. salmonea, new species

21. Perianth-tube $\mathbf{2 0 - 4 0} \mathrm{mm}$ long.

28. Plant completely glabrous; tepals ovate or lance-ovate.

29. Tepals $15 \mathrm{~mm}$ long, the outer acute; leaves $4 \mathrm{~mm}$ wide, exceeding the scape, remotely serrulate $\ldots \ldots \ldots \ldots \ldots \ldots \ldots \ldots \ldots \ldots \ldots \ldots \ldots \ldots \ldots$. glabra

29. Tepals $20 \mathrm{~mm}$ long, obtuse; leaves $7 \mathrm{~mm}$ wide, shorter than the scape, densely serrulate toward apex; leaf: furrows lacking, palisade cells

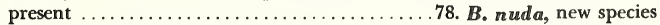

28. Plant vestite, at least partially.

30. Coronoid appendages much shorter than the anthers; tepals erect; leaf: small palisade cells present.

31. Leaf-blades pubescent; tepals to $13 \mathrm{~mm}$ long ...79. B. tomentosa

31. Leaf-blades glabrous except for the setose-ciliate margins; tepals to $23 \mathrm{~mm}$ long .............. B. cyananthera, new species

30. Coronoid appendages as long as or longer than the anthers; tepals subspreading to reflexed.

32. Free perianth-tube $10 \mathrm{~mm}$ in diameter; tepals reflexed, obtuse... $\ldots \ldots \ldots \ldots \ldots \ldots \ldots \ldots \ldots \ldots \ldots \ldots \ldots \ldots \ldots \ldots \ldots \ldots \ldots \ldots \ldots$. B. schidigera

32. Free perianth-tube, 3-6 $\mathrm{mm}$ in diameter; tepals subspreading, acute.

33. Perianth-tube densely pubescent, the free part of the perianthtube 4-6 $\mathrm{mm}$ in diameter; leaf: furrows lacking, palisade-like cells present $\ldots \ldots \ldots \ldots \ldots \ldots \ldots \ldots \ldots \ldots$. B. luzulifolia

33. Perianth-tube very sparsely pubescent and appearing glabrous; free part of perianth-tube $3 \mathrm{~mm}$ in diameter

82. B. graciliflora

13. Indument of the perianth of sessile or stipitate glands.

34. Glands of the perianth sessile or subsessile. Brazil: Minas Gerais.

35. Scape much shorter than the leaves; glands of the perianth minute and white, giving it a powdered appearance under magnification; leaf: furrows on abaxial side $\ldots \ldots \ldots \ldots \ldots \ldots \ldots \ldots \ldots \ldots \ldots \ldots \ldots \ldots \ldots \ldots \ldots \ldots \ldots \ldots \ldots$. . pulverulenta, new species

35. Scape longer than the leaves; glands of the perianth dark, evident without magnification.

36. Leaves glabrous except the margins; perianth-glands sessile; tepals $12 \mathrm{~mm}$

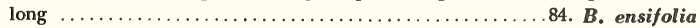

36. Leaves evenly pilose; perianth-glands subsessile; tepals $23 \mathrm{~mm}$ long .....

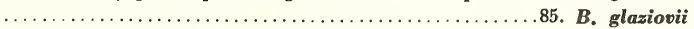

34. Glands of the perianth long-stipitate.

37. Scape exceeding the leaves, its glandular indument of 2 types. Brazil: Minas Gerais. 
38. Leaf-blades glabrous except the margins.

39. Perianth-tube 3 times as long as the ovary; leaf: furrows lacking, palisade cells lacking 86. B. conicostigma

39. Perianth-tube $4-5$ times as long as the ovary

87. B. cuspidata

38. Leaf-blades finely and evenly glandular-pubescent.

40. Flowers white; perianth-tube $40 \mathrm{~mm}$ long; tepals reflexed .........

88. B. albiflora

40. Flowers green outside; perianth-tube $50 \mathrm{~mm}$ long; tepals erect; leaf: furrows lacking, slightly elongated palisade cells present

37. Scape equaling or shorter than the leaves.

89. B. bibiriensis, new species

41. Leaf-blades glabrous except the keel and margins. Brazil: Minas Gerais.

42. Perianth-tube $65 \mathrm{~mm}$ long; flower yellow; leaf: furrows lacking, palisade cells present

90. B. longiflora

42. Perianth-tube $30-50 \mathrm{~mm}$ long.

43. Perianth-tube 5 times as long as the ovary, straight, ecostate; leaf: furrows lacking, small palisade cells present ....91. B. gardneri

43. Perianth-tube 3 times as long as the ovary, curved, costate ....... $\ldots \ldots \ldots \ldots \ldots \ldots \ldots \ldots \ldots \ldots \ldots \ldots \ldots \ldots \ldots \ldots \ldots \ldots \ldots \ldots \ldots$. curviflora

41. Leaf-blades evenly vestite.

44. Coronoid appendages linear.

45. Tepals $30-35 \mathrm{~mm}$ long; leaf: abaxial surface ridged, palisade cells present. Brazil: Minas Gerais

93. B. fragrans

45. Tepals 18-20 mm long.

46. Leaf-blades $4 \mathrm{~mm}$ wide. Brazil: Minas Gerais . 94. B. tricolor

46. Leaf-blades $10 \mathrm{~mm}$ wide. Brazil: Bahia .....95. B. blanchetti

44. Coronoid appendages broad.

47. Coronoid appendages as wide as high, quadrate or semicircular; sinus of the appendage shallow. Brazil: Minas Gerais.

48. Perianth-tube to $27 \mathrm{~mm}$ long and 4-5 $\mathrm{mm}$ wide, subdensely vestite. Brazil: Minas Gerais .............96. B. mollis

48. Perianth-tube to $65 \mathrm{~mm}$ long and $11 \mathrm{~mm}$ wide, very laxly vestite; leaf: furrows lacking, small palisade cells present. Brazil, state unknown .............. 97. B. culta, new species

47. Coronoid appendages oblong or broad-based; sinus of the appendage deep.

49. Indument of the scape white, contrasting sharply with the dark indument of the perianth-tube; perianth-tube $25 \mathrm{~mm}$ long. Brazil: Minas Gerais ............. 98. B. leucopoda

49. Indument of the scape and perianth uniform, not contrasting; perianth-tube 20-60 $\mathrm{mm}$ long.

50. Perianth and scape strongly glutinous, soon covered with dirt, bearing 2 types of trichomes; ovary much broader than the tube at anthesis; leaf: small furrows on abaxial side, palisade cells lacking. Brazil: Minas Gerais ....... $\ldots \ldots \ldots \ldots \ldots \ldots . \ldots . \ldots . \ldots . \ldots$. B. sordida, new species

50. Perianth and scape dry and clean.

51. Coronoid appendages oblong or slightly dilated at base, at least half as long as the anthers. Brazil: Minas Gerais.

52. Leaf-blades $9 \mathrm{~mm}$ wide; leaf: furrows lacking, palisade-like cells present .......100. B. blackii

52. Leaf-blades $5 \mathrm{~mm}$ wide .........101. B. rubra

51. Coronoid appendages broad-based, much contracted upward, about a third as long as the anthers.

53. Ovary rounded at base, broadly obovoid. Brazil: Minas Gerais ........102. B. damaziana

53. Ovary long-attenuate at base, slenderly obconic. Brazil: Bahia 103. B. bahiana 


\section{Subkey I}

\section{Barbacenia brachycalyx Goethart \& Henrard}

Barbacenia brachycalyx Goethart \& Henrard, Blumea, 2:340, fig. A, 1937.-L. B. Smith, Contr. U.S. Nat. Herb., 35:276, 1962.

Type.-On rocks, Pichoa to Morro do Espigão, Goiás, Brazil, Glaziou 22207 (L, holotype; B, photo 138 and $\mathrm{s} \mathrm{n)}$.

Distribution.-Known only from the typecollection.

\section{Barbacenia cylindrica L. B. Smith \& Ayensu, new species \\ Plates 1, 29a,b}

A B. brachycalyce Goethart \& Henrard, cui verisimiliter affinis, tubo perianthii valde majore et quam tepalis longioribus differt.

Caudex unknown except apex, this $13 \mathrm{~mm}$ in diameter (including leaf-bases). Leaves obscurely 3-ranked, subdensely imbricate, dry, glabrous; sheaths little if at all exposed, sulcate with distinct space between the nerves, entire; blades linear, attenuate to a filiform apex, $32 \mathrm{~cm}$ long, $8 \mathrm{~mm}$ wide, sulcate on both sides, laxly and obscurely serrulate near apex, elsewhere the teeth reduced to dark knobs.

Scape solitary, $25 \mathrm{~cm}$ long above the leaf-sheaths, glabrous. Flower glabrous, over $5 \mathrm{~cm}$ long, bright scarlet (!Anderson). Ovary subcylindric, acute at base, $18 \mathrm{~mm}$ long, $4.5 \mathrm{~mm}$ wide. Perianth-tube above ovary cylindric, flaring slightly near apex, $12 \mathrm{~mm}$ long. Tepals erect at anthesis, uniform, oblong, subacute, $23 \mathrm{~mm}$ long, $5 \mathrm{~mm}$ wide. Stamens $14 \mathrm{~mm}$ long; coronoid appendages oblong, sharply bifid, connate at base into a $4 \mathrm{~mm}$ long tube, slightly exceeding the $9 \mathrm{~mm}$ long anthers. Style shorter than the stamens, ellipsoid at apex with 3 linear apically confluent stigmas.

Leaf Anatomy (Anderson 7217).-Surface View: Hairs: none observed. Epidermis: cells square to rectangular on both surfaces; thin-walled. Stomata: tetracytic; $27 \times 18 \mu \mathrm{m}$; present in rows on both surfaces.

Transverse Section of Lamina Isolateral; very widely V-shaped. Both surfaces almost smooth. Epidermis: cells on both surfaces rounded to domeshaped; few conical; thin-walled. Cuticle: slightly thickened and smooth on entire surface. Stomata: present on both surfaces; stomata flush or slightly below epidermal surface; very small substomatal chamber present. Mesophyll: 11 or 12 layers of rounded, thin-walled cells. Cells larger and translucent in middle 4 or 5 layers of mesophyll. Vascular bundles: 45; commissural bundles observed. One or two large vessels present in each bundle, mostly one. Two phloem units lying laterally in flanges of $Y$-shaped abaxial girder which extends to the abaxial epidermis. Inverted $Y$-shaped adaxial girder present on each bundle and extends to adaxial epidermis. Bundle sheath completely surrounding each bundle. Crystals: few present in mesophyll. Tannins: present.

TYPE.-By stream in shrubby woods, Chapada dos Veadeiros, $13 \mathrm{~km}$ by road south of Terezina, ca. $1000 \mathrm{~m}$ alt, 16 March 1973, Anderson 7217 (US, holotype; NY; UB, isotypes).

Distribution.-Known only from the typecollection.

\section{Barbacenia spiralis L. B. Smith \& Ayensu, new species \\ Plates 2, 29c,}

A $B$. brachycalyce Goethart \& Henrard, cui verisimiliter affinis, foliorum laminis spiraliter recurvatis, tubo perianthii late obconico, tepalis multo majoribus differt.

Caudex decumbent, to $5 \mathrm{dm}$ long (!Irwin et al.), $15 \mathrm{~mm}$ thick (including old leaf-sheaths). Leaves apparently much more than 3-ranked, very densely imbricate; sheaths completely hidden until very old and shattered; blades recurving in tight spirals, linear-lanceolate, filiform-attenuate, $10 \mathrm{~cm}$ long, 14 $\mathrm{mm}$ wide, laxly ciliate toward base with spreading setae $5 \mathrm{~mm}$ long, elsewhere glabrous, sulcate on both sides.

Scape solitary, $9 \mathrm{~cm}$ long above the leaf-sheaths, glabrous. Flower (immature) over $5 \mathrm{~cm}$ long, glabrous, dull red (!Irwin et al.). Ovary broadly obconic, $12 \mathrm{~mm}$ high. Perianth-tube (whole) broadly obconic, blending with lines of ovary, $16 \mathrm{~mm}$ high. Tepals linear-lanceolate, acute, $5 \mathrm{~cm}$ long in bud. Stamens as long as the tepals in bud; anthers 18 
mm long; filaments free; coronoid appendages oblong, erose at summit, $25 \mathrm{~mm}$ long. Style and stigma not observed.

LEAF ANATOMy (Irwin et al. 27642).-Surface View: Hairs: none observed. Epidermis: cells on both surfaces square to rectangular; few with more sides; thin-walled. Stomata: tetracytic; $21 \times 12 \mu \mathrm{m}$; present in rows on both surfaces.

Transverse Section of Lamina: ? Isolateral; almost horizontal. Both surfaces slightly ridged, especially adaxial. Few tufts of hairs at extreme margin of blade. Epidermis: cells on both surfaces rounded to dome-shaped; thin-walled. Cuticle: thick and ridged over entire surface. Stomata: present on both surfaces; stomata slightly sunken; small substomatal chamber present. Mesophyll: consisting of layers of rounded to elongated cells on adaxial side followed by 6 or 7 layers of rounded, translucent, or obliterated cells; below this are 4 or 5 layers of rounded, thin-walled cells. Cells large and translucent above midvein. Vascular bundles: 51; commissural bundles observed. One large vessel present in each bundle. Two phloem units lying laterally in flanges of $U$-shaped abaxial girder. Thick, inverted $Y$-shaped adaxial girder present on each bundle. Girders extend to epidermis on both sides. Bundle sheath completely surrounding each bundle. Crystals: few present in mesophyll. Tannins: few observed.

Note: The mesophyll cells often break down and become obliterated in mature leaves especially in the intercostal region.

TYPE.-On outcrop in cerrado, ca. $8 \mathrm{~km}$ east of Diamantina on road to Extração, Serra do Espinhaço, Minas Gerais, Brazil, $1175 \mathrm{~m}$ alt, 16 March 1970, Irwin et al. 27642 (NY, holotype).

Distribution.-Known only from the typecollection.

\section{Barbacenia beauverdii Damazio}

Barbacenia beauverdii Damazio, Bull. Herb. Boiss., ser. 2, 595, unnumbered fig. 1907.-L. B. Smith, Contr. U.S. Nat. Herb., 35:276, 1962.

TYPE.-Among rocks, near Arrial de Antonio Pereira, Serra do Frasão, Minas Gerais, Brazil, 1100 m alt, 26 March 1907, L. Damazio 1846 (G. holotype).

Distribution.-Known only from the typecollection.

\section{Barbacenia pabstiana L. B. Smith \& Ayensu, new species}

Plates 3, 29e,f

A B. caricina Goethart \& Henrard, cui affinis, floribus violaceis vel azureis, antheris appendicibus coronoideis subaequantibus differt.

Caudex decumbent (?), simple or branched, 5-9 $\mathrm{cm}$ long, ca. $5 \mathrm{~mm}$ thick (including leaf-sheaths). Leaves clearly 3-ranked, glabrous; sheaths exposed up to $5 \mathrm{~mm}$, somewhat viscid; blades linear, filiform-attenuate, to $18 \mathrm{~cm}$ long, $3-4 \mathrm{~mm}$ wide, the thick yellowish margin laxly serrulate.

Scape solitary, 13-19 cm long above the leafsheaths, laxly setulose. Flower ca. $3 \mathrm{~cm}$ long, laxly setulose at base and more or less on ribs, elsewhere glabrous, blue to violet. Ovary cylindric becoming ellipsoid, to $12 \mathrm{~mm}$ long. Perianth-tube contracted above ovary into a 2-3 $\mathrm{mm}$ cylinder. Tepals divergent at anthesis, $15 \mathrm{~mm}$ long, elliptic, the inner distinctly broader than the outer. Stamens slightly exceeding the broadly spatulate bilobed $7 \mathrm{~mm}$ long coronoid appendages. Style slenderly clavate at apex with 3 linear apically confluent stigmas.

Leaf Anatomy (Hatschbach \& Ahumada 31360) -Surface View: Hairs: none observed. Epidermis: cells on both surfaces square to rectangular; thinwalled. Stomata: anomocytic with 4-6 subsidiary cells, mostly $4 ; 24 \times 21 \mu \mathrm{m}$; present in rows on both surfaces.

Transverse Section of Lamina: Dorsiventral; V-shaped with small but sharp median adaxial groove. Surface gently undulating but some areas clearly grooved. Epidermis: cells on both surfaces rounded to dome-shaped; thin-walled. One layer of rounded cells subjacent to adaxial epidermis. Cuticle: thickened and slightly ridged over entire surface. Stomata: present on both surfaces; stomata flush with epidermal surface; substomatal chamber present. Mesophyll: 3 or 4 layers of palisade cells followed by 6 or 7 layers of rounded or rectangular thin-walled cells, some cells translucent, others idioblastic. Many cells filled with chlorophyll in chloroplasts. Vascular bundles: 41; commissural bundles observed. One or two vessels present in each bundle, mostly one. Two phloem units lying laterally in flanges of $Y$-shaped abaxial girder. Inverted $Y$-shaped adaxial girder present on each bundle, except midvein. Bundle sheath completely 
surrounding each vascular bundle. Crystals: few solitary crystals observed. Tannins: few present.

Type.-Cliffs of hillside, Aracê, Mun. Domingos Martins, Espírito Santo, Brazil, 800-1000 m alt, 7 February 1973, Hatschbach \& Ahumada 31360 (US, holotype; MBM, isotype).

Distribution.-Brazil, Espírito Santo: Pedra Azul, $1000 \mathrm{~m}$ alt, 24 August 1970, Kautsky 279 (HB, US) .

\section{Barbacenia caricina Goethart \& Henrard}

Barbacenia caricina Goethart \& Henrard, Blumea, 2:340, fig. B, 1937.-L. B. Smith, Contr. U.S. Nat. Herb., 35:276, 1962.

Type.-Pedra do Conego, Nova Friburgo, Minas Gerais, Brazil, 23 June 1880, Glaziou 12222 (L, holotype; B, photo s n, 158).

Distribution.-Known only from the typecollection.

\section{Barbacenia delicatula L. B. Smith \& Ayensu, new species}

Plates 4, 30a,b

A B. caricina Goethart \& Henrard, cui affinis, floribus minoribus, appendicibus coronoideis antheras multo superantibus differt.

Caudex decumbent, simple or branched, 19 (-32) cm long, ca. $5 \mathrm{~mm}$ thick (including the leaf-sheaths). Leaves obscurely 3-ranked, subdensely imbricate, strongly sulcate on both sides, dry, glabrous, sheaths apically much exposed; blades linear, filiformattenuate, $17 \mathrm{~cm}$ long, $3(-5.5) \mathrm{mm}$ wide, serrulate on margins and keel especially near apex.

Scape solitary, 4-5 cm long above the leaf-sheaths, glabrous. Flower glabrous, greenish white. Ovary ellipsoid, $6 \mathrm{~mm}$ long. Perianth-tube above ovary cylindric, $2 \mathrm{~mm}$ long. Tepals divergent at anthesis, uniform, elliptic, $11 \mathrm{~mm}$ long. Stamens attached near base of appendage; anthers $3 \mathrm{~mm}$ long; coronoid appendages oblong, $7 \mathrm{~mm}$ long, shortbifid with rounded lobes. Style shorter than the coronoid appendages, slenderly clavate at apex with 3 linear apically confluent stigmas.

Leaf Anatomy.-Ayensu, Smithsonian Contr. Bot., 15:53, pl. 46f, 1974.

TYPE.-On shaded cliff by Rio Santo Antônio,
Route MG-2, Conceição do Mato Dentro, Minas Gerais, Brazil, 18 January 1972, L. B. Smith, Ayensu \& Hatschbach (separate no. 28878) 15975 (US, holotype; MBM, isotype).

Distribution.-Brazil, Minas Gerais: typelocality, 8 August 1972, Hatschbach 30090 (MBM, US); 27 October 1974, Hatschbach \& Ferreira (MBM, US).

\section{Barbacenia squamata Herbert}

Barbacenia squamata Herbert, Bot. Reg., 29, Misc.: 64, 1843.Paxton, Mag. Bot., 11:75, 76, 1844.-Hooker, Bot. Mag., 71, pl. 4136, 1845.-Seubert in Martius, Fl. Bras., 3(1):68, 1847. -L. B. Smith, Contr. U.S. Nat. Herb., 35:276, 1962.Strang, Taxon, 16:69, 1967.

Vellozia squamata Jackson, Ind. Kew., 4:1269, 1895 [wrongly attributed to Bentham \& Hooker f., Gen. 3:740, 1883, who made the combination only inferentially and not validly].

Type--Organ Mountains, Rio de Janeiro, Brazil, Veitch Hortus $s$, from Lobb in 1841 (K, holotype (?), not seen).

Distribution.-Known only from the typecollection.

\section{Barbacenia rogieri hortus ex Moore \& Ayres}

Barbacenia rogieri hortus ex Moore \& Ayres, Mag. Bot., 2:209, pl., 1850.-Lemaire, Jard. Fl., 1: pl. 82, 1851.-L. B. Smith, Contr. U.S. Nat. Herb., 35:276, 1962.

TYPE.-South America, Van Houtte Hortus s $n$ (holotype ?). Based on the original description and plate until type-material is located.

Distribution.-Evidently the same species from Mangaratiba, Rio de Janeiro, Brazil, 20 January 1962 Martins 260 (GUA subsequently lost, US photo).

\section{Barbacenia seubertiana Goethart \& Henrard}

Barbacenia seubertiana Goethart \& Henrard, Blumea, 2:360, 1937.-L. B. Smith, Contr. U.S. Nat. Herb., 35:276, 1962.Ayensu, Smithsonian Contr. Bot. 15:56, fig. 19a-c, 38c-e, 1974.

Barbacenia purpurea Hooker var. minor Seubert in Martius, F1. Bras. 3(1):68, 1847 [type: Serra dos Orgãos, Rio de Janeiro, Brazil, Gardner 5897 (K, holotype, not seen)].

TYPE.-On rock, summit of Serra dos Orgãos, Rio de Janeiro, Brazil, 6 March 1888, Glaziou 17824 (L, holotype; B, photo s n). 
Distribution.-Brazil: Rio de Janeiro city and state.

\section{Barbacenia gaveensis Goethart \& Henrard}

Barbacenia gaveensis Goethart \& Henrard, Blumea, 2:346, 1937.-L. B. Smith, Contr. U.S. Nat. Herb., 35:276, 1962.Ayensu, Smithsonian Contr. Bot., 15:55, fig. 18a-c, 1974.

Type.-Pedra da Gavea, Rio de Janeiro city, Brazil, 29 June 1875, Glaziou s $n$ (L, holotype; B, photo 152).

Distribution.-Known only from the typelocality.

\section{Barbacenia stenophylla Goethart \& Henrard}

Barbacenia stenophylla Goethart \& Henrard, Blumea, 2:361, 1937.-L. B. Smith, Contr. U.S. Nat. Herb., 35:276, 1962 .Ayensu, Smithsonian Contr. Bot., 15:57, 1i,j, 19d-f, 1974.

TYPE.-Among rocks, Morro do Salto, Goiás, Brazil, 15 January 1895, Glaziou 22220a (L, holotype, B, photo 154).

Distribution.-Brazil: Minas Gerais: in soil-filled crevices of outcrop, $6 \mathrm{~km}$ south of Cristalina, Serra dos Cristais, $17^{\circ} \mathrm{S}, 48^{\circ} \mathrm{W}$, Goiás, Brazil, $1175 \mathrm{~m}$ alt, 4 November 1965, Irwin et al. 9901 (NY, UB, US).

\section{Barbacenia inclinata Goethart \& Henrard}

Barbacenia inclinata Goethart \& Henrard, Blumea, 2:352, 1937.-L. B. Smith, Contr. U.S. Nat. Herb., 35:276, 1962.

TYPE.-On rocks, Serra dos Cristaes, near Diamantina, Minas Gerais, Brazil, 4 April 1892, Glaziou 19921 (L, holotype; B, photo s n, 150, 176).

Distribution.-Known only from the typecollection.

\section{Barbacenia longiscapa Goethart \& Henrard}

Barbacenia longiscapa Goethart \& Henrard, Blumea, 2:355, 1937.-L. B. Smith, Contr. U.S. Nat. Herb., 35:276, 1962.Ayensu, Smithsonian Contr. Bot., 15:55, pl. 47d, 1974.

TyPE.-Wet rocks, Serra da Lapa (Serra do Cipó), Minas Gerais, Brazil, December 1824, Riedel 1062 (L, holotype; B, photo s n, 140, 177, 178).

Distribution.-Brazil: Minas Gerais: rocky depressions, $12 \mathrm{~km}$ southwest of Diamantina, $1370 \mathrm{~m}$ alt, 18 January 1969, Irwin et al. 22186 (NY, UB, US).

15. Barbacenia hatschbachii L. B. Smith \& Ayensu, new species

Plates 5, 30c, $d$

A B. longiscapa Goethart \& Henrard, cui valde affinis, foliis longioribus angustioribusque scapos superantibus differt.

Caudex simple (?), incompletely known, ca. $2 \mathrm{~cm}$ long (?), $12 \mathrm{~mm}$ thick (including the leaf-sheaths). Leaves obscurely 3-ranked, subdensely imbricate, strongly sulcate on both sides, glabrous; sheaths apically much exposed, dark brown dorsally, somewhat viscous; blades linear, filiform-attenuate, 40 $50 \mathrm{~cm}$ long, 5-9 mm wide, serrulate on margins and keel especially toward apex, pale beneath.

Scapes 1-3 (or the caudex very obscurely branched?), 24-30 cm long above the leaf-sheaths, laxly and minutely stipitate-glandular toward apex. Flower red (typically) to greenish white, laxly glandular toward base. Ovary ellipsoid, $6 \mathrm{~mm}$ long at anthesis. Perianth-tube above ovary broadly funnelform, $2 \mathrm{~mm}$ long. Tepals divergent at anthesis, uniform, narrowly elliptic (2-) $4 \mathrm{~cm}$ long. Stamens attached near apex of appendage and anther exceeding it; coronoid appendage narrowly spatulate with short broadly rounded lobes. Style exceeding the stamens, clavate at apex with 3 linear apically confluent stigmas.

Leaf Anatomy.-Ayensu, Smithsonian Contr. Bot., 15:54, pl. 21a, 47b, 1974.

Type.-Sandstone cliffs, Serra de Maracajú, Mun. Aquidauana, Mato Grosso, Brazil, 16 May 1970, Hatschbach 24284 (US, holotype; MBM, isotype).

Distribution.-Brazil: Mato Grosso: typelocality, 18 February 1970, Hatschbach 23765 (MBM, US);. 19 April 1972, Hatschbach 29607 (MBM).

\section{Barbacenia monticola L. B. Smith \& Ayensu, new species}

Plates 6, 30e,f

A B. longiscapa Goethart \& Henrard, cui verisimiliter affinis, foliis angustioribus, floribus minoribus, appendicibus coronoideis oblongis antheras subaequantibus differt. 
Caudex at least $7 \mathrm{~cm}$ long, simple, ca. $6 \mathrm{~mm}$ thick (including the leaf-sheaths). Leaves 3-ranked, subdensely imbricate, strongly sulcate on both sides, dry, glabrous; sheaths apically much exposed, dark brown beneath; blades linear, finely attenuate, $9 \mathrm{~cm}$ long, $4 \mathrm{~mm}$ wide, serrulate on margins and keel especially near apex.

Scape solitary, $23 \mathrm{~cm}$ long above the leaf-sheaths laxly stipitate-glandular. Flower red (!Hatschbach), laxly vestite with dark stipitate glands. Ovary ellipsoid $5 \mathrm{~mm}$ long. Perianth-tube above ovary broadly funnel-form, $5 \mathrm{~mm}$ long. Tepals divergent at anthesis, uniform, narrowly elliptic, $10 \mathrm{~mm}$ long. Anthers nearly equaling the appendages; coronoid appendages oblong with broadly rounded lobes, 4.5 $\mathrm{mm}$ long. Style about equaling the appendages, slenderly clavate at apex.

Leaf Anatomy (Hatschbach \& Ahumada 31402). -Surface View: Hairs: few tufts present on edge of lamina. Epidermis: cells square to rectangular on adaxial; thin-walled. Abaxial cells square to rectangular; irregular among stomata; thin-walled. Stomata: tetracytic; $24 \times 15 \mu \mathrm{m}$; present in rows on abaxial surface.

Transverse Section of Lamina: Dorsiventral; widely $V$-shaped with extreme margins turned downward. Both surfaces slightly undulating. Epidermis: cells on both surfaces rounded to domeshaped; thin-walled. Layer of rounded parenchyma subjacent to adaxial epidermis. Cuticle: very thick and only slightly ridged on adaxial side; thick and ridged on abaxial side. Stomata: present on abaxial surface only; stomata flush with epidermal surface; substomatal chamber present. Mesophyll: five layers of small palisade-like cells followed by six layers of rounded cells. Many mesophyll cells devoid of chloroplasts; cells translucent above midvein. Vascular bundles: 27; commissural bundles observed. One or two large vessels present in each bundle, mostly one. Two phloem units lying laterally in flanges of $Y$-shaped abaxial girder. Inverted $Y$-shaped adaxial girder present on each bundle. Some girders extending laterally along the epidermis. Bundle sheath completely surrounding each bundle. Crystals: none observed. Tannins: many present.

TyPe.-Wet cliffs, Parque Nacional de Caparao, Mun. Caparao, Minas Gerais, Brazil, 16 February 1973, Hatschbach \& Ahumada 31402 (US, holotype, MBM, isotype).
Distribution.-Known only from the typelocality.

\section{Barbacenia flavida Goethart \& Henrard}

Barbacenia flavida Goethart \& Henrard, Blumea, 2:343, 1937. -L. B. Smith, Contr. U.S. Nat. Herb., 35:276, 1962.

TYPE.-On rocks, Pichoa, Morro do Espigão, Goiás, Brazil, 5 October 1894, Glaziou 22207 bis (L, holotype; B, photo).

Distribution.-Known only from the typecollection.

18. Barbacenia irwiniana L. B. Smith

Barbacenia irwiniana L. B. Smith, Contr. U.S. Nat. Herb., 35:276, pl. 10: figs. 17, 18, 1962.-Ayensu, Smithsonian Contr. Bot., 15:55, pl. 48a, 1974.

TYPE.-In narrow crevice among rocks on steep slope, Pico da Bandeira, Serra Caparao, Minas Gerais, Brazil, $2700 \mathrm{~m}$ alt, 3 March 1959, Irwin 2803 (US, holotype; NY, isotype).

Distribution.-Known only from the typelocality.

\section{Barbacenia foliosa Goethart \& Henrard}

Barbacenia foliosa Goethart \& Henrard, Blumea, 2:344, fig. D, 1937.-L. B. Smith, Contr. U.S. Nat. Herb., 35:277, 1962.

Trpe.-On rocks, Tijuca, Rio de Janeiro (city), Brazil, November 1894, Ule 4056 (B, holotype; B, photo).

Distribution.-Known only from the typecollection.

20. Barbacenia burle-marxii L. B. Smith \& Ayensu, new species

Plates 7, 31 $a, b$

A B. foliosa Goethart \& Henrard, cui affinis, foliorum laminis subduplo-latioribus, tepalis rotundatis apiculatisque differt.

Caudex not known. Leaves strongly sulcate on both sides, dry, glabrous; blades linear, finely attenuate, $20 \mathrm{~cm}$ long, $4 \mathrm{~mm}$ wide, involute when dry, 
serrulate on margins and keel especially toward apex.

Scape $24 \mathrm{~cm}$ long above leaf-sheaths, laxly vestite with minute pale stipitate glands. Flowers purple. Ovary narrowly turbinate, $14 \mathrm{~mm}$ long. Perianthtube (whole) narrowly turbinate, $20 \mathrm{~mm}$ long, laxly and minutely pale-glandular. Anthers nearly equaling the appendages; coronoid appendages oblong with broadly rounded lobes, $9 \mathrm{~mm}$ long. Style exceeded by the stamens, apically subulate with 3 linear apically confluent stigmas.

Leaf ANatomy (L. B. Smith \& Ayensu 16014).Surface View: Hairs: few tufts on edge of lamina. Epidermis: cells on both surfaces square to rectangular; thin-walled. Stomata: anomocytic with 4-6 subsidiary cells, mostly $4 ; 24 \times 12 \mu \mathrm{m}$; present in rows on adaxial and abaxial surfaces.

Transverse Section of Lamina: Dorsiventral; widely $V$-shaped with extreme margins turned slightly inversely. Both surfaces slightly undulating, but some areas distinctly grooved. Epidermis: cells on both surfaces small; rounded to dome-shaped; thin-walled. Subjacent to epidermis occurs a layer of rounded, thin-walled parenchyma cells. Cuticle: thickened and slightly ridged on both surfaces. Stomata: present on both surfaces; fairly large substomatal chamber present; stomata flush with epidermal surface. Mesophyll: 4 or 5 layers of palisade-like cells; many cells idioblastic filled with tannin. Palisade-like cells followed by 5 or 6 layers of rounded, compactly arranged spongy cells. Few translucent cells above midvein. Vascular bundles: 33; commissural bundles observed. One or two large vessels present in each bundle; mostly one. Two phloem units present in flanges of abaxial girder. Both adaxial and abaxial girders very thin; $Y$-shaped with long stem extending laterally below epidermis. Bundle sheath completely surrounding each bundle. Crystals: few in mesophyll. Tannins: present in mesophyll.

TyPE.-Cultivated by Roberto Burle-Marx, 30 January 1972, originally from Venda Nova, Espírito Santo, Brazil, L. B. Smith \& Ayensu 16014 (US, holotype).

Distribution.-Known only from the typecollection.

\section{Barbacenia goethartii Henrard}

Barbacenia goethartii Henrard, Blumea, 2:350, fig. K, 1937.L. B. Smith, Contr. U.S. Nat. Herb., 35:277, 1962.
Type.-Penha (?), Minas Gerais, Brazil, St.Hilaire s $n$ (P, holotype; $\mathrm{B}$, photo 136).

Distribution.-Known only from the typecollection.

\section{Barbacenia brevifolia Taubert}

Barbacenia brevifolia Taubert, Bot. Jahrb. 12, Beibl. 27:2, 1890.

\section{2a. Barbacenia brevifolia var. brevifolia}

Barbacenia brevifolia var. brevifolia.-L. B. Smith, Contr. U.S. Nat. Herb., 35:277, 1962.

Leaf-blades straight, flat, $2-4 \mathrm{~mm}$ wide. Flowers $3 \mathrm{~cm}$ long.

TYPE.-Brazil, Glaziou 13271 (B, holotype(?); C, isotype; F, photo 22311).

Distribution.-Known only from the typecollection.

\section{2b. Barbacenia brevifolia var. recurvata}

\section{B. Smith}

Barbacenia brevifolia var. recurvata L. B. Smith, Contr. U.S. Nat. Herb., 35:290, 1962.

Leaf-blades arching-recurved, ca. $8 \mathrm{~mm}$ wide. Flowers $4 \mathrm{~cm}$ long.

TyPE.-On rocks, Guinda, Mun. Diamantina, Minas Gerais, Brazil, 5 November 1937, Mello Barreto 9526 (NY, holotype).

Distribution.-Known only from the typecollection.

\section{Barbacenia ionantha L. B. Smith}

Barbacenia ionantha L. B. Smith, Phytologia, 9:262, figs. 3-6, 1963.

Type.-Near the two lakes, above the stone house, Serra do Caparaó, Minas Gerais, Brazil, 2 March 1960, Flávia Tórgo 14 (HB, holotype).

Distribution.-Known only from the typecollection.

\section{Barbacenia mantiqueirae Goethart \& Henrard}

Barbacenia mantiqueirae Goethart \& Henrard, Blumea, 2:355, 1937.-L. B. Smith, Contr. U.S. Nat. Herb., 35:277, 1962. 
TyPe.-Pico dos Marins, Serra da Mantiqueira, São Paulo, Brazil, 10 January 1897, Löfgren in Herb. Commissão Geogr. \& Geol. S. Paulo 3570 (L, holotype; B, photo $179,180,181,182$ ).

Distribution.-Known only from the typecollection.

\section{Barbacenia spectabilis L. B. Smith \& Ayensu, new species}

Plates 8, 31 c,d

A B. rogieri hortus ex Moore \& Ayres, cui affinis, floribus majoribus, ovarii costis multis dense glandulosis differt.

Caudex 8-18 cm long, 20-25 mm thick (including leaf-sheaths). Leaves glabrous, sulcate on both sides; sheaths slightly exposed, brownish, somewhat viscous; blades linear, filiform-attenuate, to $60 \mathrm{~cm}$ long, $14 \mathrm{~mm}$ wide, dry, laxly serrulate throughout the dark margins and the keel.

Scapes 3 or 4 , to $50 \mathrm{~cm}$ long above the leafsheaths, laxly dark-glandular. Flowers purple. Ovary ellipsoid, 15-20 $\mathrm{mm}$ long. Perianth-tube densely vestite on the numerous costae with dark stipitate glands, above the ovary narrowed into a cylinder 2-3 $\mathrm{mm}$ high. Tepals to $6 \mathrm{~cm}$ long, undulatemargined (!Menezes), the outer oblong, ca. $12 \mathrm{~mm}$ wide living (!Menezes), the inner lanceolate, $25 \mathrm{~mm}$ wide living (!Menezes). Anthers $25 \mathrm{~mm}$ long; coronoid appendages subspatulate, the lobes short, subacute, slightly exceeding the anthers. Style subulate with a long sterile apex and median stigmas.

Leaf Anatomy (Menezes \& Sazima 57).-Surface View: Hairs: none observed. Epidermis: cells on both surfaces square to rectangular; thin-walled. Stomata: tetracytic; $21 \times 18 \mu \mathrm{m}$; present in rows on both surfaces.

Transverse Section of Lamina: Dorsiventral or ?isolateral; widely V-shaped, almost horizontal. Both surfaces slightly ridged; more so on adaxial surface. Epidermis: cells on both surfaces rounded to dome-shaped; thin-walled. One layer of thinwalled, rounded parenchyma subjacent to adaxial epidermis. Cuticle: slightly thickened and ridged over entire surface. Stomata: present on both surfaces, mostly on abaxial surface; stomata flush with epidermal surface; large subsidiary cells flanking stomata; substomatal chamber present. Mesophyll: 7 or 8 layers of small palisade cells; first 1-4 layers containing few translucent cells. This is followed by 5 or 6 layers of rounded, thin-walled, obliterated cells; below this is 4 layers of rounded, thin-walled cells. Translucent cells with wavy walls above broadly extended midvein. Vascular bundles: 75; commissural bundles observed. One large xylem vessel present in each bundle. Two phloem units lying laterally in flanges of $\mathrm{Y}$-shaped abaxial girder. Inverted $Y$-shaped adaxial girder present on each bundle. Both girders slightly extended laterally along epidermis. Bundle sheath completely surrounding each bundle. Crystals: none observed. Tannins: few present.

Note: Obliterated mesophyll cells occur near abaxial surface. The sclerenchyma below midvein is conspicuously extended.

Floral Anatomy.-Menezes, Bol. Zool. \& Biol. Mar., ser. 2, no. 30:721, 732, 733 (fig.), 1973.

Type--Morro de Agá, Piuma, Espírito Santo, Brazil, 10 July 1967, Oliveira Filho \& Dias 3 (US, holotype; SP, isotype).

Distribution.-Brazil: Espírito Santo: typelocality, 8 August 1969, Menezes \& Sazima 56 (SPF, US); 57 (SPF, US).

\section{Barbacenia nigrimarginata L. B. Smith}

Barbacenia nigrimarginata L. B. Smith, Contr. U.S. Nat. Herb., 35:277, pl. 10: figs. 19-22, 1962.

Aylthonia nigrimarginata (L. B. Smith) Menezes, Ciência e Cultura, 23(3):422, 1971.

TrPE.-Stony summit of butte shoulder $5 \mathrm{~km}$ west of Veadeiros, Chapada dos Veadeiros, ca. $14^{\circ} 30^{\prime} \mathrm{S}, 47^{\circ} 30^{\prime} \mathrm{W}$, Goiás, Brazil, 29 April 1956, Dawson 14717 (US, holotype).

Distribution.-Brazil: Goiás: Veadeiros.

\section{Barbacenia gounelleana Beauverd}

Barbacenia gounelleana Beauverd, Bull. Herb. Boiss. ser. 2, 7:704, fig. 8, 1907.-L. B. Smith, Contr. U.S. Nat. Herb., 35:277, 1962.-Ayensu, Smithsonian Contr. Bot., 15:53, figs. la,b, 20d-f, pls. 6d, 38f-h, 51b, 1974.

TyPE.-On rocks, Sitio de Ramos, Itatiaia, Rio de Janeiro, Brazil, $2400 \mathrm{~m}$ alt, February 1899, Gounelle s $n$ ( $\mathrm{G}$, holotype).

Distribution.-Brazil: Rio de Janeiro: Mount Itatiaia. 


\section{Barbacenia purpurea Hooker}

Barbacenia purpurea Hooker, Bot. Mag., 54, pl. 2777, 1827.Seubert in Martius, FI. Bras., 3(1):67, pl. 8: fig. 7, 1847.L. B. Smith, Contr. U.S. Nat. Herb., 35:278, 1962.-Ayensu, Smithsonian Contr. Bot., 15:55, fig. 18d-f, pl. 39a-c, 1974.

Pleurostima purpurea (Hooker) Rafinesque, Fl. Tellur., 2:97, 1836.

Type-Brazil, cultivated in England, August 1827, Hooker from Herbert (K, holotype(?) or lacking this the original description and plate).

Distribution.-Brazil: Rio de Janeiro (city).

29. Barbacenia andersonii L. B. Smith \& Ayensu, new species

Plates 9, 31e,f

A B. purpurea, cui verisimiliter affinis, tepalis obtusis, appendicium lobis dilatatis rotundatisque differt.

Caudex (incompletely known) erect (?), simple, over $8 \mathrm{~cm}$ or to $40 \mathrm{~cm}$ long (!Anderson-[plant] 70 $\mathrm{cm}$ tall), $8 \mathrm{~mm}$ thick including leaf-sheaths. Leaves 3-ranked, sulcate on both sides, glutinous; sheaths apically exposed for $5 \mathrm{~mm}$, dark brown beneath; blades very narrowly triangular, filiform-attenuate, $15 \mathrm{~cm}$ long, $8 \mathrm{~mm}$ wide, serrulate on margins and keel.

Scapes 1 or $2,32 \mathrm{~cm}$ long (above the leaf-sheaths) laxly vestite with minute dark stipitate glands. Flowers purple, dark-glandular. Ovary ellipsoid, 10 $\mathrm{mm}$ long; perianth-tube above ovary cylindric, 2 $\mathrm{mm}$ long. Anthers $12 \mathrm{~mm}$ long; coronoid appendages spatulate, bifurcate with rounded lobes, $22 \mathrm{~mm}$ long. Style subulate with subapical stigmas.

Leaf Anatomy (Anderson 6704).-Surface View: Hairs: few tufts present on outer edges of lamina. Epidermis: adaxial and abaxial cells square to rectangular; thin-walled; in long rows across the surface; interspersed with few rounded areas of irregularly shaped cells. Stomata: tetracytic; $27 \times$ $21 \mu \mathrm{m}$; present only in abaxial furrows.

Transverse Section of Lamina: Dorsiventral ? isolateral; V-shaped with small median adaxial groove. Both surfaces slightly undulating with slight furrows on abaxial surface. Conspicuous grooves occur on adaxial side of bundles. Epidermis: cells on both surfaces rounded to dome-shaped; few conical; thin-walled. Subjacent to epidermis is one layer of rounded, thin-walled parenchyma cells.
Adaxial parenchyma larger above midvein; few sclerenchyma cells present in adaxial parenchyma layer. Cuticle: slightly thickened and undulating on both surfaces. Stomata: present in abaxial furrows only; outer and inner ledges present; stomata flush with epidermal surface; large substomatal chamber present. Mesophyll: five layers of elongated cells followed by 7 or 8 layers of rounded, compactly arranged cells. Vascular bundles: 65; commissural bundles observed. One or two large vessels present in each bundle; mostly one. Two phloem units lying laterally in flanges of $Y$-shaped abaxial girder. Inverted $\mathrm{Y}$-shaped adaxial girder present on each bundle. Midvein has no adaxial girder, but has conspicuously rounded abaxial sclerenchyma. Bundle sheath completely surrounding each bundle. Crystals: none observed. Tannins: few present in mesophyll.

TyPE.-In cerrado with sandstone outcrops, 27 km by road north of Alto Paraíso, Goiás, Brazil, $1750 \mathrm{~m}$ alt, 9 March 1973, Anderson 6704 (US, holotype; NY; UB, isotypes).

Distribution.-Known only from the typecollection.

\section{Barbacenia celiae Maguire}

Barbacenia celiae Maguire, Mem. New York Bot. Gard., 18(2):33, fig. 6 A-N, 1969.-Ayensu, Smithsonian Contr. Bot., 15:57, pl. 48b, 1974 .

TYPE.-Common on conglomerate outcrop, northwest escarpment of Serra Tepequem, Roraima, Brazil, 860-960 m alt, 23 November 1954, Maguire \& Maguire 40025 (NY, holotype; US, isotype).

Distribution.-Brazil: Roraima: Serra Tepequem, Serra Sabang.

\section{Subkey II}

\section{Barbacenia involucrata L. B. Smith}

Barbacenia involucrata L. B. Smith, Phytologia, 9:263, figs. 8-10, 1963.-Ayensu, Smithsonian Contr. Bot., 15:58, fig. $1 \mathrm{k}-1$, pls. 20b, 47c, 1974.

TyPE.-Serra do Cipó, Minas Gerais, Brazil, 3 March 1958, Heringer \& Castellanos 5944 (US, holotype; $\mathrm{UB}$, isotype).

Distribution.-Brazil: Minas Gerais: Serra do Cipó, Almeida, Jaboticatubas. 


\section{Barbacenia schwackei Goethart \& Henrard}

Barbacenia schwackei Goethart \& Henrard, Blumea, 2:359, fig. J, 1937.-L. B. Smith, Contr. U.S. Nat. Herb., 35:278, 1962.-Ayensu, Smithsonian Contr. Bot., 15:58, fig. 2la-c, pl. 39d, 1974.

Type--Serra do Cipó, Minas Gerais, Brazil, 25 April 1892, Schwacke 8357 (B, holotype; B, photo 125).

Distribution.-Known only from the typecollection.

\section{Barbacenia fulva Goethart \& Henrard}

Barbacenia fulva Goethart \& Henrard, Blumea, 2:345, fig. E, 1937.-L. B. Smith, Contr. U.S. Nat. Herb., 35:278, 1962.

Type.-Minas Gerais, Brazil, St.-Hilaire $C^{\boldsymbol{1}} 362$ (P, holotype; B, photo 122).

Distribution.-Known only from the typecollection.

\section{Barbacenia polyantha Goethart \& Henrard}

Barbacenia polyantha Goethart \& Henrard, Blumea, 2:357, 1937.-L. B. Smith, Contr. U.S. Nat. Herb., 35:278, 1962.

Type.-Among rocks, Pinheiro, near Biribiri, Mun. Diamantina, Minas Gerais, Brazil, 26 May 1892, Glaziou 19931 (L, holotype; B, photo s n, 113).

Distribution.-Known only from the typecollection.

\section{Barbacenia trigona Goethart \& Henrard}

Barbacenia trigona Goethart \& Henrard, Blumea, 2:362, 1937.-L. B. Smith, Contr. U.S. Nat. Herb., 35:278, 1962.

TYPE.-Milho Verde (ca $18^{\circ} 27^{\prime} \mathrm{S}, 4^{\circ} 30^{\circ} \mathrm{W}$ ), Minas Gerais, Brazil, St.-Hilaire 489 (P, holotype; B, photo 117).

Distribution.-Known only from the typecollection.

\section{Barbacenia globata Goethart \& Henrard}

Barbacenia globata Goethart \& Henrard, Blumea, 2:349, 1937.-L. B. Smith, Contr. U.S. Nat. Herb., 35:278, 1962.

TYPE.-On rocks of mountain by "Rio dos Pedros," Minas Gerais, Brazil, 28 April 1892, Schwacke 8359 (B, holotype; B, photo 110).
Distribution.-Known only from the typecollection.

\section{Barbacenia coccinea Martius ex Schultes f.}

Barbacenia coccinea Martius ex Schultes f, in Roemer and Schultes, Syst., 7:286, 1826.-Seubert in Martius, F1. Bras., 3(1):71, pl. 8: fig. 4, 1847.-L. B. Smith, Contr. U.S. Nat. Herb., 35:278, 1962.

Type--On mountains, Minas Gerais, Brazil, Martius s $n$ (M, holotype; F, photo 18989).

Distribution.-Known only from the typecollection.

\section{Barbacenia glutinosa Goethart \& Henrard}

Barbacenia glutinosa Goethart \& Henrard, Blumea, 2:350, 1937.-L. B. Smith, Contr. U.S. Nat. Herb., 35:278, 1962.

Type-_Arenos, "M. da Lapa" (Serra do Cipó), Minas Gerais, Brazil, November-December 1824, Riedel 1060 (L, holotype; B, photo s n, 127).

Distribution.-Known only from the typecollection.

\section{Barbacenia markgrafii Schulze-Menz}

Barbacenia markgrafii Schulze-Menz in Markgraf, Notizblatt, 15:216, 1940.-L. B. Smith, Contr. U.S. Nat. Herb., 35:278, 1962.

Type.-Sandy open riverbank forest, mountains east of Montes Claros, Grão Mogul, Rio Itacambirussu, Minas Gerais, Brazil, $700 \mathrm{~m}$ alt, 13 November 1938, Markgraf, Brade \& Mello Barreto 3519 (B, holotype).

Distribution.-Known only from the typecollection.

\section{Barbacenia ignea Martius ex Schultes $\mathrm{f}$.}

Barbacenia ignea Martius ex Schultes f. in Roemer and Schultes, Syst., 7:285, 1826.-Seubert in Martius, Fl. Bras., 3(1):71, pl. 8: fig. 6, 1847.-L. B. Smith, Contr. U.S. Nat. Herb., 35:278, 1962.-Ayensu, Smithsonian Contr. Bot., 15:58, fig. 2ld-f, pls. $39 \mathrm{e}, \mathrm{f}, 40 \mathrm{a}, 1974$.

TyPe.-On mountains, Minas Gerais, Brazil, Martius s $n$ (M, holotype; F, photo 18994).

Distribution.-Known only from the typecollection. 


\section{Barbacenia lilacina Goethart \& Henrard}

Barbacenia lilacina Goethart \& Henrard, Blumea, 2:354, 1937.-L. B. Smith, Contr. U.S. Nat. Herb., 35:278, 1962.

\section{1a. Barbacenia lilacina var. lilacina}

Leaf-blades hirsute. Flowers lilac. Coronoid appendages bifid $1 / 3^{-1 / 2}$.

Type.-On rocks, Serra do Funil, by Rio Paranaha, Minas Gerais, Brazil, 20 April 1892, Glaziou 19926 (L, holotype; B, photo s n, 128, 129).

Distribution.-Known only from the typecollection.

\section{1b. Barbacenia lilacina var. pallidiflora Henrard ex L. B. Smith}

Barbacenia lilacina var. pallidiflora Henrard, Blumea, 2:354, 1937 [nomen illeg.].-Henrard ex L. B. Smith, Contr. U.S. Nat. Herb., 35:278, 1962.

Leaf-blades sparsely hirsute. Flowers pale lilac. Coronoid appendages bifid more than $1 / 2$.

TYPE.-In campo, Curalhino near Diamantina, Minas Gerais, Brazil, 14 April 1892, Glaziou 19925 (L, holotype).

Distribution.-Known only from the typecollection.

\section{Barbacenia vandellii Pohl ex Seubert}

Barbacenia vandellii Pohl ex Seubert in Martius, Fl. Bras., 3(1):72, pl. 8: fig. 3, 1847.-L. B. Smith, Contr. U.S. Nat. Herb., 35:278, 1962.-Ayensu, Smithsonian Contr. Bot., 15:61, figs. le,f, 22a-c, 1974.

Type.-São João, Minas Gerais, Brazil, Pohl s $n$, Lhotsky s $n$ (B, paratypes, destroyed(?), $\mathrm{n}$ v).

Drstributıon.-Brazil: Minas Gerais: São João, Itambé (Serra do Cipó).

\section{Barbacenia brasiliensis Willdenow}

Barbacenia brasiliensis Willdenow, Sp. Pl., 2:227, 1799.L. B. Smith, Contr. U.S. Nat. Herb., 35:278, 1962.

Barbacenia Vandelli, Fl. Lusit. \& Brasil. Spec., 21, pl. 1, 1788.-Roemer, Script. Hisp., 98, pl. 6: fig. 9, 1796 [without indication of species].

Barbacenia bicolor Martius, Nov. Gen. \& Sp., 1:19, pl. 13, 1823.-Seubert in Martius, Fl. Bras. 3(1):72, 1847 [type: Martius s $n$ (M, holotype, $\mathrm{n} v$ ), rocky and grassy mountain slopes, Diamantina, Minas Gerais, Brazil, $1200 \mathrm{~m}$ alt.].
Barbacenia vandelli Schultes f. in Roemer \& Schultes, Syst., $7: 288,1826$ [wrongly attributed to Roemer who cited the genus and its author without any specific name].

Visnea Steudel ex Endlicher, Gen., 173, 1837 [nomen in synonymy, without indication of species].

Type.-Brazil, collector(?) (holotype?). Based on Vandelli and Roemer illustrations in default of specimens.

Distribution.-Brazil: Minas Gerais: Diamantina.

44. Barbacenia nanuzae L. B. Smith \& Ayensu, new species

Plates 10, 32a

A B. hirtiflora Goethart \& Henrard, cui affinis, floribus gracilibus subduplo longioribus differt.

Caudex not known. Leaf-blades linear, filiformattenuate, $30 \mathrm{~cm}$ long, $14 \mathrm{~mm}$ wide, laxly setoseciliate, sulcate on both sides, otherwise smooth and glabrous.

Scapes to $26 \mathrm{~cm}$ long (complete), densely vestite with fine crisped mostly nonglandular hairs. Ovary subcylindric, ca. $30 \mathrm{~mm}$ long. Perianth-tube densely vestite with fine stipitate glands, the upper ellipsoid and slightly dilated from the ovary at anthesis, 18 $\mathrm{mm}$ long. Anther $7 \mathrm{~mm}$ long, much shorter than the sharply bidentate coronoid appendage. Style about equaling the appendages, the elliptic stigmas about $9 \mathrm{~mm}$ below the subulate sterile apex.

LeAf АNAтомy (partial leaf material) (Menezes 265).-Surface View: Hairs: few tufts on edge of lamina. Epidermis: cells on both surfaces square to rectangular; thin-walled. Stomata: paracytic and tetracytic; $24 \times 18 \mu \mathrm{m}$; present in rows on both surfaces.

Transverse Section of Lamina: Isolateral; both surfaces very ridged with conspicuous grooves. Epidermis: cells on both surfaces rounded to domeshaped; thin-walled. Cuticle: thick and very ridged on both surfaces. Stomata: present on both surfaces, mostly present on abaxial surface; stomata usually slightly below surface; small substomatal chamber observed. Mesophyll: 14 layers of large rounded, thin-walled cells. Vascular bundles: total unknown; commissural bundles observed. One or two large vessels present in each bundle. Two phloem units lying laterally in flanges of thick, Y-shaped abaxial girder. Thick, inverted $Y$-shaped adaxial girder 
present. Bundle sheath completely surrounding each bundle. Crystals: few present. Tannins: present in mesophyll.

TYPE.-Km 114 on the route from Lagoa Santa to Conceição do Mato Dentro, Serra do Cipó, Minas Gerais, Brazil, 10 September 1972, Menezes 265 (US, holotype; SPF, isotype).

Distribution.-Known only from the typecollection.

\section{Barbacenia hirtiflora Goethart \& Henrard}

Barbacenia hirtiflora Goethart \& Henrard, Blumea, 2:351, fig. L, 1937.-L. B. Smith, Contr. U.S. Nat. Herb, 35:278, 1962.

Type.-On rocks, Curalhino, Diamantina, Minas Gerais, Brazil, 14 April 1892, Glaziou 19927a (B, holotype; B, photo 126).

Distribution.-Known only from the typecollection.

\section{Barbacenia williamsii L. B. Smith}

Barbacenia williamsii L. B. Smith, Contr. U.S. Nat. Herb. 35:278, pl. 10: figs. 23, 24, 1962.-Ayensu, Smithsonian Contr. Bot., 15:61, fig. 22d-f, pl. 40b-d, 1974.

Type--On iron ore, Serra da Mutuca, Mun. Nova Lima, Minas Gerais, Brazil, 1200-1400 m alt, 7 April 1945, Williams \& Assis $6696(\mathrm{GH}$, holotype; US, isotype).

Distribution.-Brazil: Minas Gerais: Serra do Curral and Serra da Mutuca in Nova Lima.

\section{Barbacenia flava Martius ex Schultes $\mathrm{f}$.}

Barbacenia flava Martius ex Schultes f. in Roemer \& Schultes, Syst., 7:286, 1826.-Seubert in Martius, Fl. Bras., 3(1):71, 1847.-L. B. Smith, Contr. U.S. Nat. Herb., 35:279, 1962.Ayensu, Smithsonian Contr. Bot., 15:57, figs. 1c-d, 23a-c, pls. $6 \mathrm{a}, \mathrm{b}, 40 \mathrm{e}-\mathrm{g}, 1974$.

\section{7a. Barbacenia flava var. flava}

Caudex to $8 \mathrm{~cm}$ long. Leaf-blades $13-20 \mathrm{~cm}$ long, 8-12 mm wide. Scapes equaling the leaves. Perianthtube $16 \mathrm{~mm}$ long. Tepals $10-12 \mathrm{~mm}$ long.

Type.-On rocks, summit of Serra de Itambé,
Minas Gerais, Brazil, $1800 \mathrm{~m}$ alt, Martius s $n$ (M, holotype), according to Schultes filius; summit of Serra Branca, Capivari, Martius s $n$ (M, F, photo 18992). At present we have no explanation for this discrepancy.

Distribution.-Brazil: Minas Gerais: Diamantina, Jaboticatubas, Itambé, Itabirito, Mato Dentro, Capivari.

47b. Barbacenia flava var. minor $\mathrm{L}$. B. Smith

Barbacenia flava var. minor L. B. Smith, Contr. U.S. Nat. Herb., 35:279, 1962.

Caudex 1-4 cm long. Leaf-blades $8-13 \mathrm{~cm}$ long, to $8 \mathrm{~mm}$ wide. Scapes shorter than the leaves. Perianth-tube $15-20 \mathrm{~mm}$ long. Tepals $5 \mathrm{~mm}$ long.

Type-Ca. 31/2 miles from Hotel Chapeu do Sol, Serra do Cipó, Mun. Jaboticatubas, Minas Gerais, Brazil, $1100 \mathrm{~m}$ alt, 19 December 1959, Maguire, Maguire \& Pires 44665 (US, holotype; $\mathrm{N}$, isotype).

Distribution.-Brazil: Minas Gerais: Jaboticatubas.

48. Barbacenia glauca Martius ex Schultes $\mathrm{f}$.

Barbacenia glauca Martius ex Schultes f. in Roemer \& Schultes, Syst., 7:288, 1826.-Seubert in Martius, F1. Bras., 3(1):72, 1847.-L. B. Smith, Contr. U.S. Nat. Herb., 35:279, 1962.

TyPE.-Summit of Serra de Itambé, Minas Gerais, Brazil, Martius s $n$ (M, holotype; F, photo 18993).

Distribution.-Known only from the typecollection. Doubtfully distinct from B. flava Martius ex Schultes $\mathrm{f}$.

\section{Barbacenia itabirensis Goethart \& Henrard}

Barbacenia itabirensis Goethart \& Henrard, Blumea, 2:353, 1937.-L. B. Smith, Contr. U.S. Nat. Herb., 35:279, 1962.

Type.-Among rocks, Pico d'Itabira do Campo, Minas Gerais, Brazil, 20 December 1888, Glaziou 17827 (B, holotype).

Distribution.-Known only from the typecollection. 


\section{Barbacenia sellovii Goethart \& Henrard}

Barbacenia sellovii Goethart \& Henrard, Blumea, 2:359, 1937.-L. B. Smith, Contr. U.S. Nat. Herb., 35:279, 1962.

Type.-Serra da Mooda (Moeda?), Minas Gerais, Brazil, 1818, Sellow C 311 (L, holotype; W, isotype; F, photo 30022).

Distribution.-Brazil: Minas Gerais: Serra da Moeda, Serra da Piedade, Serra do Cipó (?).

\section{Subkey III}

\section{Barbacenia macrantha Lemaire}

Barbacenia macrantha Lemaire, Jard. Fl., 4:pl. 390, 1854.L. B. Smith, Contr. U.S. Nat. Herb., 35:279, 1962.-Ayensu, Smithsonian Contr. Bot., 15:65, fig. 2f, pls. 8a,b, 48d, 1974. Aylthonia macrantha (Lemaire) Menezes, Ciência e Cultura, 23(3):[421], 1971.

TYPE.-On rocks, Diamantina, Serra do Cipó, Minas Gerais, Brazil, Libon $s$, cultivated by $D e$ Jonghe (BR(?) $\mathrm{n} v$, in the absence of any specimen amply typified by the original description and illustration).

Distribution.-Brazil: Minas Gerais: Diamantina, Conceição do Mato Dentro.

\section{Barbacenia rubro-virens Martius}

Barbacenia rubro-virens Martius, Nov. Gen. \& Sp., 1:20, pl. 14, fig. 1, 1823.-Seubert in Martius, F1. Bras., 3(1):68, 1847. -L. B. Smith, Contr. U.S. Nat. Herb., 35:279, 1962.

TYPE.-On rock, Diamantina, Minas Gerais, Brazil, Martius s $n$ (M, holotype, $\mathrm{n}$ v). In default of specimen, excellently typified by original description and illustration.

Distribution.-Brazil: Minas Gerais: Diamantina.

\section{Barbacenia riedeliana Goethart \& Henrard}

Barbacenia riedeliana Goethart \& Henrard, Blumea, 2:358, 1937.-L. B. Smith, Contr. U.S. Nat. Herb., 35:280, 1962.Ayensu, Smithsonian Contr. Bot., 15:67, pls. 9b, 47g, 1974.

Trpe.-Serra da Lapa (Serra do Cipó), Minas Gerais, Brazil, November 1824, Riedel 1058 (L, holotype; B, photo 130).

Distribution.-Brazil: Minas Gerais: Diamantina, Gouvêa.
54. Barbacenia chlorantha L. B. Smith \& Ayensu, new species

Plates 11, 32b,c

A B. riedeliana Goethart \& Henrard, cui affinis, tubo perianthii ampliore, ovario vix distincto, appendicibus coronoideis suboblongis differt.

Caudex $25 \mathrm{~mm}$ thick (including leaf-sheaths). Leaf-blades very narrowly triangular, filiformacuminate, $13 \mathrm{~cm}$ long, $6 \mathrm{~mm}$ wide, laxly setoseciliate, sulcate on both sides.

Scapes $2,9 \mathrm{~cm}$ long above the leaf-sheaths, densely vestite with finely stipitate pale glands. Ovary slenderly turbinate, $10 \mathrm{~mm}$ long. Perianth-tube (whole) subcylindric flaring slightly at apex, $65 \mathrm{~mm}$ long, $13 \mathrm{~mm}$ wide at apex, green (!Anderson et al.). Tepals nearly uniform, lanceolate, acute, $20 \mathrm{~mm}$ long, yellow (!Anderson et al.). Anthers $11 \mathrm{~mm}$ long, slightly exceeding the oblong sharply bidentate coronoid appendages. Style about equaling the appendages, ovoid at apex with 3 linear apically confluent stigmas.

LeAf Anatomy (Anderson et al. 35488).-Surface View: Hairs: few very long tufts present at edge of lamina. Epidermis: cells on both surfaces square to rectangular; thin-walled. Stomata: tetracytic; $21 \times$ $18 \mu \mathrm{m}$; present in rows on both surfaces.

Transverse Section of Lamina: Dorsiventral; widely V-shaped. Both surfaces gently undulating. Epidermis: cells on both surfaces small; rounded to dome-shaped; thin-walled. Cuticle: thick and ridged on both surfaces. Stomata: present on both surfaces; stomata flush with epidermal surface; small substomatal chamber present. Mesophyll: 5 or 6 layers of small palisade cells; many translucent, especially just above spongy tissue. This is followed by 5 or 6 layers of compactly arranged rounded cells. Cells translucent and fairly large above midvein. Vascular bundles: 45; commissural bundles observed. One or two large vessels present in each bundle. Two phloem units lying in flanges of thick $\mathrm{U}$ - or $\mathrm{Y}$-shaped abaxial girder. Thick, inverted $\mathrm{U}$ - or $Y$-shaped adaxial girder present on each bundle. Girders extending to both surfaces. No adaxial girder on midvein but abaxial crescent-shaped sclerenchyma present. Crystals: raphide bundles and druses present in most of mesophyll. Tannins: few present.

TyPE.-Steep sandstone cliffs and open rock hillsides, ca. $14 \mathrm{~km}$ southwest of Diamantina on road to 
Gouvêa, Minas Gerais, Brazil, 1300-1360 m alt, 5 February 1972, Anderson, Stieber of Kirkbride 35488 (UB, holotype; US, photo).

Distribution.-Known only from the typecollection.

\section{Barbacenia luzulifolia Martius ex Schultes f.}

Barbacenia luzulifolia Martiuus ex Schultes f. in Roemer \& Schultes, Syst., 7:285, 1826.-Seubert in Martius, Fl. Bras., 3(1):69, pl. 8: fig. 2, 1847,-L. B. Smith, Contr. U.S. Nat. Herb., 35:280, 1962.-Ayensu, Smithsonian Contr. Bot., 15:65, fig. 1o,p, pls. 6c, 47e, 1974.

Aylthonia luzulifolia (Martius ex Schultes f.) Menezes, Ciência e Cultura, 23(3):[421], 1971.

TyPE.- "Villa Ricca" (Ouro Preto), Minas Gerais, Brazil, Martius s $n$ (M, holotype; F, photo 18996).

Distribution.-Brazil: Minas Gerais: Caete (Serra da Piedade), Ouro Preto.

56. Barbacenia saxicola L. B. Smith \& Ayensu, new species

Plates $12,32 d$

A B. luzulifolia Martius ex Schultes f., cui parum affinis, appendicibus coronoideis subovatis obtuse lobatis quam antheris subtriplo brevioribus differt.

Caudex at least $4 \mathrm{~cm}$ long, covered by reflexed leaf-blades. Leaves sulcate on both sides, viscous; sheaths not visible; blades linear, filiform-attenuate, over $11 \mathrm{~cm}$ long, $8 \mathrm{~mm}$ wide, laxly setose-ciliate.

Scape single, $11 \mathrm{~cm}$ long above the leaf-sheaths, laxly vestite with fine contorted partly tumid-based hairs. Flowers reddish orange (!Anderson et al.), hairy like the scape. Ovary cylindric, $13 \mathrm{~mm}$ long, $6 \mathrm{~mm}$ wide. Perianth-tube slenderly infundibuliform, blending with the ovary, $45 \mathrm{~mm}$ long, $11 \mathrm{~mm}$ wide at apex. Tepals lanceolate, $20 \mathrm{~mm}$ long, the outer narrow, completely tomentose, the inner broad, tomentose only on the median base. Anthers $15 \mathrm{~mm}$ long. Coronoid appendages ovate with short obtuse lobes, $6 \mathrm{~mm}$ long. Style not enlarged at apex; stigmas linear, apically confluent.

Leaf Anatomy (partial leaf specimen) (Anderson et al 35344).-Surface View: Hairs: none observed. Epidermis: cells on both surfaces square to rectangular; thin-walled. Stomata: tetracytic; $21 \times 18$ $\mu \mathrm{m}$; present in rows on both surfaces.

Transverse Section of Lamina: Dorsiventral;
V-shaped. Both surfaces undulating. Epidermis: cells on both surfaces rounded to dome-shaped; thin-walled. Cuticle: thickened and ridged over entire surface. Stomata: present on both surfaces; stomata sunken below the epidermal surface and flanked by 2 protuberances; substomatal chamber present. Mesophyll: 4 or 5 layers of small palisade cells followed by 6 or 7 layers of rounded, thinwalled cells; many cells appear translucent. Vascular bundles: total unknown; commissural bundles observed. One large vessel present in each bundle. Two phloem units lying laterally in flanges of wide Y-shaped abaxial girder. Thin, inverted Y-shaped adaxial girder present on each bundle. Girders extend to epidermis on both sides. Bundle sheath completely surrounding each bundle. Crystals: few present in mesophyll. Tannins: present, especially in epidermis and bundle sheath.

TYPE.-On rocks (sandstone), cerrado and open hillsides, $8 \mathrm{~km}$ north of Gouvêa on road to Diamantina, Minas Gerais, Brazil, $1220 \mathrm{~m}$ alt, 4 February 1972, Anderson, Stieber o Kirkbride 35344 (US, holotype; NY; UB, isotypes).

Distribution.-Known only from the typecollection.

\section{Barbacenia reflexa L. B. Smith \& Ayensu, new species}

Plates 13, 32e,f

Barbacenia rubro-virens sensu L. B. Smith in Ayensu, Smithsonian Contr. Bot., 15:67, fig. 1m, n, 23d-f, 1974 [in part, as to Maguire, Magalhaes \& Maguire 49260].

A B. luzulifolia Martius ex Schultes f., cui verisimiliter affinis, foliis scapisque multo majoribus, tepalis angustis reflexis differt.

Caudex (incomplete) over $2 \mathrm{~cm}$ long, ca. $2 \mathrm{~cm}$ thick with leaf-sheaths. Leaves sulcate on both sides, appearing dry but not certain because of fire damage; sheaths completely covered; blades linear, filiform-attenuate, $25 \mathrm{~cm}$ long, $8 \mathrm{~mm}$ wide, laxly setose-ciliate near base, otherwise glabrous.

Scape single, $23 \mathrm{~cm}$ long above leaf-sheaths, subdensely brown-tomentose. Flowers mauve-tinged (!Maguire et al.), subdensely brown-tomentose. Ovary narrowly turbinate, $18 \mathrm{~mm}$ long, $9 \mathrm{~mm}$ wide, narrowly infundibuliform, slightly constricted above ovary, $12 \mathrm{~mm}$ wide at apex. Anthers $9.5 \mathrm{~mm}$ long; coronoid appendages narrowly subtriangular, 
acutely bidentate. Style not enlarged at apex; stigmas linear, apically confluent.

Leaf Anatomy.-Ayensu, Smithsonian Contr. Bot., 15:67, fig. Im,n, 23d-f, 1974, sub B. rubrovirens.

TYPE.-On sandstone, slopes and summit of Serra Grão Mogul (16 ${ }^{\circ} 39^{\prime} \mathrm{S}$, 42 $\left.45^{\prime} \mathrm{W}\right)$, Minas Gerais, Brazil, 900-1100 m alt, 17 August 1960, Maguire, Magalhães \& Maguire 49260 (US, holotype; NY, isotype).

Distribution.-Known only from the typecollection.

Note: This collection was originally determined as $B$. rubro-virens Martius and may well be more nearly related to that species than our admittedly artificial key indicates.

\section{Barbacenia graminifolia L. B. Smith}

Barbacenia graminifolia L. B. Smith, Phytologia, 9:263, figs. 11-14, 1963.

Type.-Serra do Cipó, Minas Gerais, Brazil, 3 March 1958, Heringer \& Castellanos 5950 (UB, holotype; US, isotype).

Distribution.-Known only from the typecollection.

\section{Barbacenia latifolia L. B. Smith \& Ayensu, new species}

Plates 14, 33a,b

A B. viscosissima Goethart \& Henrard, cui affinis, foliis latioribus, perianthii tubo ampliore differt.

Caudex (incomplete) stout but completely covered by reflexed leaves. Leaf-blades linear-lanceolate, filiform-attenuate, somewhat contracted toward base, $18 \mathrm{~cm}$ long, $23 \mathrm{~mm}$ wide centrally, sulcate and laxly short-pubescent on both sides, ciliate with fine gland-tipped setae.

Scapes 2, $12 \mathrm{~cm}$ long above the leaf-sheaths, densely vestite with fine gland-tipped crisped hairs. Flowers white (!Duarte \& Graziela), vestite like the scape. Ovary ellipsoid, distinct, $18 \mathrm{~mm}$ long. Perianth-tube above ovary infundibuliform, ca. 20 $\mathrm{mm}$ long, $5 \mathrm{~mm}$ wide at base, $15 \mathrm{~mm}$ wide at apex. Tepals lanceolate, over $30 \mathrm{~mm}$ long. Anthers 15 $\mathrm{mm}$ long. Coronoid appendages suboblong, acutely bidentate, $25 \mathrm{~mm}$ long. Style (extreme apex missing) fusiform-enlarged in the upper half; stigmas linear.

Leaf Anatomy (Duarte \& Graziela 7884).-Surface View: Hairs: long, multicellular and tufts present on both surfaces. Epidermis: cells on both surfaces 4- to 6-sided; mostly rectangular; thinwalled. Stomata: tetracytic; $21 \times 18 \mu \mathrm{m}$; present on both surfaces.

Transverse Section of Lamina: Dorsiventral; very widely V-shaped; almost horizontal. Long multicellular hairs and tufts of hairs present on both surfaces. Both surfaces conspicuously ridged. Epidermis: cells on both surfaces rounded to dome-shaped; few conical; thinwalled; large above midvein. Cuticle: fairly thin and smooth over entire surface. Stomata: present on both surfaces; stomata usually raised above epidermal surface; substomatal chamber present. Mesophyll: 4 or 5 layers of small, rounded cells followed by 1 or 2 layers of large, translucent cells. Below is 4 or 5 layers of small, rounded cells. Cells above midvein large and translucent. Vascular bundles: 51; commissural bundles observed. One or two large vessels present in each bundle, mostly one. Two phloem units lying laterally in flanges of short Y-shaped abaxial girder. Inverted $Y$-shaped adaxial girder present. Girders extend to epidermis. Crystals: druses present in most of mesophyll. Tannins: present.

Note: The regions of the blade between vascular bundles are greatly reduced, thus giving the leaf the appearance of grass anatomy.

TYPE.-Rocky ground, Rio dos Cristaes, Minas Gerais, Brazil, 15 January 1963, Duarte \& Graziela [Barroso] 7884 (US, holotype; RB, isotype).

Distribution.-Known only from the typecollection.

\section{Barbacenia paranaensis L. B. Smith}

Barbacenia paranaensis L. B. Smith, Contr. U.S. Nat. Herb., 35:280, pl. 10: figs. 25, 26, 1962.-Ayensu, Smithsonian Contr. Bot., 15:66, pls. 20a, 48e, 1974.

Aylthonia paranaensis (L. B. Smith) Menezes, Ciência e Cultura, 23(3):422, 1971.

TYPE.-On sandstone (arenite) bluffs, Fazenda Morungava, Rio de Funil, Mun. Sengés, Paraná, Brazil, I5 December 1958, Hatschbach \& Lange 5359 (US, holotype; MBM, isotype). 
Distribution.-Brazil: Paraná: Ortigueira, Sengés.

\section{Barbacenia viscosissima Goethart \& Henrard}

Barbacenia viscosissima Goethart \& Henrard, Blumea, 2:363, 1937.-L. B. Smith, Contr. U.S. Nat. Herb., 35:280, 1962.Ayensu, Smithsonian Contr. Bot., 15:69, pls. 40h, 4la,b, 1974.

Aylthonia viscosissima (Goethart \& Henrard) Menezes, Ciência e Cultura, 23(3):422, 1971.

Type-—São João (del Rei), Minas Gerais, Brazil, June 1824, Riedel 205 (L, holotype; LE, isotype).

Distribution.-Brazil: Minas Gerais: Ouro Preto, São João del Rei.

\section{Barbacenia umbrosa L. B. Smith \& Ayensu, new species}

Plates 15, 33c,d

A B. viscosissima Goethart \& Henrard, cui affinis, foliorum laminis angustioribus scapos subaequantibus supra basin glabris differt.

Caudex (incomplete) over $2 \mathrm{~cm}$ long, $23 \mathrm{~mm}$ wide including leaf-sheaths but wholly covered by reflexed blades. Leaves to 24 in terminal fascicle, sulcate on both sides; sheaths brown and lustrous beneath, glabrous where not exposed; blades linear, filiform-attenuate, to $17 \mathrm{~cm}$ long, $6 \mathrm{~mm}$ wide, whitepubescent and laxly setose-ciliate near base, elsewhere glabrous, sulcate on both sides.

Scapes 2 or 3 , to $16 \mathrm{~cm}$ long, laxly and finely stipitate-glandular. Flowers dull red (!Irwin et al.), laxly glandular like the scape. Ovary ellipsoid, distinct, $10 \mathrm{~mm}$ long. Perianth-tube above ovary slenderly infundibuliform, 35-40 mm long, $4 \mathrm{~mm}$ wide at base, $10 \mathrm{~mm}$ at apex. Tepals nearly uniform, linear-lanceolate, $17 \mathrm{~mm}$ long. Anthers ca. 11 $\mathrm{mm}$ long. Coronoid appendages subtriangular, sharply bidentate, ca. $7 \mathrm{~mm}$ long. Style subglobose at apex; stigmas elliptic, apically confluent.

LeAF ANATOMY (Irwin et al. 23491).-Surface View: Hairs: multicellular, present on abaxial surface. Epidermis: cells on both surfaces square to rectangular; thin-walled. Stomata: tetracytic; $18 \times$ $15 \mu \mathrm{m}$; present in rows on both surfaces.

Transverse Section of Lamina: Dorsiventral; widely V-shaped with margins turned slightly downward. Epidermis: cells on both surfaces rounded to dome-shaped; thin-walled. Cuticle: thick and ridged over entire surface. Stomata: present on both surfaces, mostly on abaxial surface; stomata flush with epidermal surface; small substomatal chamber present. Mesophyll: 3 or 4 layers of small palisadelike cells followed by 2 or 3 layers of large rounded cells. Subjacent to these occur 4 layers of small rounded cells. Many cells translucent, especially above midvein. Vascular bundles: 27; commissural bundles observed. One large vessel present in each bundle. Two phloem units lying laterally in flanges of thick, Y-shaped abaxial girder. Slightly thin, inverted $Y$-shaped adaxial girder present on each bundle. Girders extend to epidermis on both sides. Each bundle completely surrounded by a bundle sheath; cells very large near middle of bundle. Crystals: few present in mesophyll. Tannins: present, especially in epidermis.

TYPE.-In partially shaded crevices of outcrops, sandstone gorge with steep slopes, supporting cerrado and low woods, ca. $5 \mathrm{~km}$ north of Grão Mogul, Minas Gerais, Brazil, $1000 \mathrm{~m}$ alt, 18 February 1969, Irwin et al. 23491 (NY, holotype; US, photo).

Distribution.-Known only from the typecollection.

\section{Barbacenia exscapa Martius}

Barbacenia exscapa Martius, Nov. Gen. \& Sp., 1:21, pl. 14, 1823.-Seubert in Martius, Fl. Bras., 3(1):69, 1847.-L. B. Smith, Contr. U.S. Nat. Herb., 35:280, 1962.-Ayensu, Smithsonian Contr. Bot., 15:63, pl. 46g, 1974.

Aylthonia exscapa (Martius) Menezes, Ciência e Cultura, 23(3):[421], 1971 [as “excapa”].

TYPE.-Among rocks of granular micaceous schist, summit of Itambé da Villa do Principe (Sêrro), Minas Gerais, Brazil, Martius s n (M, holotype; F, photo 18991).

Distribution.-Brazil: Minas Gerais: Peak of Itambé, Sêrro.

\section{Barbacenia rectifolia L. B. Smith \& Ayensu, new species}

Plates 16, 33e,

A B. exscapa Martius, cui valde affinis, foliorum laminis rectis angustioribus laxe inconspicueque denticulatis differt. 
Caudex simple, 2 to over $5 \mathrm{~cm}$ long. Leaf-sheaths completely covered; blades erect to reflexed, straight, flat, linear, filiform-attenuate, $16 \mathrm{~cm}$ long, 6-8 mm wide, laxly ciliate with minute bristletipped teeth, sulcate on both sides, otherwise smooth and glabrous.

Scape single, very short and completely hidden by the leaf-bases. Flower appearing sessile. Ovary turbinate, ca. $5 \mathrm{~mm}$ long, not distinct. Perianthtube (whole) subcylindric, $40 \mathrm{~mm}$ long, $5 \mathrm{~mm}$ wide, very sparsely white-pubescent, green (!Anderson et al.). Tepals nearly uniform, elliptic, obtuse, $12 \mathrm{~mm}$ long, yellow (!Anderson et al.). Anthers $9 \mathrm{~mm}$ long, extending into the perianth-tube; coronoid appendages elliptic, $9 \mathrm{~mm}$ long, the 2 lobes short, subacute. Style subglobose at apex; stigmas linear, apically confluent.

Leaf Anatomy (Anderson et al. 35674).- Surface View: Hairs: none observed. Epidermis: cells on both surfaces square to rectangular; walls thickened. Stomata: tetracytic; $24 \times 21 \mu \mathrm{m}$; present in rows on abaxial surface.

Transverse Section of Lamina: Dorsiventral; widely V-shaped with recurved margins. Both surfaces gently undulating. Epidermis: cells on both surfaces rounded to dome-shaped; walls thickened. Subjacent to adaxial epidermis is 2 or 3 layers of rounded, thin-walled cells. Few sclerenchyma fibers present here. Adaxial epidermis and subjacent layers often large above midvein. Cuticle: thick and ridged on both surfaces. Stomata: present on abaxial surface only; stomata flush with surface; small substomatal chamber present. Mesophyll: three layers of elongated, palisade cells followed by 10 or 11 layers of rounded, compactly arranged cells. Vascular bundles: 25; commissural bundles observed. One or two large vessels present in each bundle. Two phloem units lying laterally in flanges of thin $Y$-shaped abaxial girder. Thin, inverted Y-shaped adaxial girder present. Abaxial girder extends to epidermis; adaxial girder extends to cells subjacent to epidermis. Each bundle surrounded by a prominent bundle sheath. Crystals: present in most mesophyll cells. Tannins: present.

TYPE.-Matted on sandstone rocks by river, southeastern drainage of Pico Itambé, about $5 \mathrm{~km}$ directly west and north of Santo Antonio de Itambé, Minas Gerais, Brazil, $950 \mathrm{~m}$ alt, 9 February 1972, Anderson, Stieber do Kirkbride 35674 (US, holotype; NY; UB, isotypes).
Distribution.-Known only from the typecollection.

\section{Barbacenia aurea L. B. Smith \& Ayensu, new species}

Plates 17, 34a,b

A B. sessiliflora L. B. Smith, cui affinis, foliis angustioribus albo-pubescentibus, floribus aureis, ovario vix distincto differt.

Caudex to $10 \mathrm{~cm}$ high, ca. $2 \mathrm{~cm}$ thick including old leaf-sheaths. Leaves sulcate on both sides; sheaths completely covered; blades linear-lanceolate, filiform-attenuate, $9 \mathrm{~cm}$ long, $10 \mathrm{~mm}$ wide, subdensely white-pubescent throughout, finely setoseciliate.

Scape not observed. Flower single, largely concealed by leaves and appearing sessile, densely white-pubescent, yellow-orange (!Anderson et al). Ovary $5 \mathrm{~mm}$ long, scarcely distinct. Perianth-tube (whole) cylindric, slightly flaring at apex, $32 \mathrm{~mm}$ long, $3 \mathrm{~mm}$ wide. Tepals oblong, obtuse, $10 \mathrm{~mm}$ long. Anthers $12 \mathrm{~mm}$ long, extending downward into the perianth-tube; coronoid appendages subovate, bidentate with short subacute lobes, $2.5 \mathrm{~mm}$ long. Style about equaling the anthers; stigmas not observed.

Leaf ANatomy (Irwin et al. 29046).-Surface View: Hairs: long, multicellular, present on both surfaces. Epidermis: cells square to rectangular on both surfaces; thin-walled; cells in rows. Stomata: tetracytic; $21 \times 18 \mu \mathrm{m}$; present on abaxial surface only.

Transverse Section of Lamina: Dorsiventral; widely V-shaped. Both surfaces gently undulating. Multicellular hairs present on both surfaces. Epidermis: cells on both surfaces rounded to domeshaped and conical; thin-walled. Subjacent to epidermis occurs a layer of thin-walled parenchyma cells. Cuticle: thin and smooth over entire surface. Stomata: present on abaxial surface; fairly large substomatal chamber present; stomata flush with epidermal surface. Mesophyll: 2 or 3 layers of palisade cells followed by 5 or 6 layers of rounded spongy tissue. Two or three layers of translucent cells above midvein. Mesophyll cells thin-walled. Vascular bundles: 45; commissural bundles not observed. One or two large vessels present in each bundle. Two phloem units lying laterally in flanges 
of short U- or $\mathrm{V}$-shaped abaxial girder extending to abaxial epidermis. Inverted $Y$-shaped adaxial girder present on each bundle; girder extends to adaxial surface and forms a T-shape beneath epidermis. Conspicuous bundle sheath completely surrounding each vascular bundle. Crystals: druses and styloids present in most of mesophyll. Tannins: few present in mesophyll.

Type.-On sandstone outcrops in cerrado, ca. 10 km west of Barão de Cocais, Minas Gerais, Brazil, $1500 \mathrm{~m}$ alt, 24 January 1971, Irwin, Harley bे Omishi 29046 (US, holotype; NY; UB, isotypes).

Distribution.-Known only from the typecollection.

\section{Barbacenia coronata P. F. Ravenna, new species}

Plates $18,34 c, d$

A B. sessiliflora L. B. Smith, cui affinis, foliorum laminis obscure denticulatis, ovario vix distincto differt.

Caudex erect (?), to $15 \mathrm{~cm}$ high, simple or shortbranched at apex, ca. $12 \mathrm{~mm}$ thick with leaf-bases. Leaves sulcate on both sides; sheaths completely hidden; blades linear, filiform-attenuate, $13 \mathrm{~cm}$ long, $4 \mathrm{~mm}$ wide, obscurely denticulate, glabrous.

Scapes solitary, to $20 \mathrm{~mm}$ long, very slender, completely hidden by the leaves. Flowers appearing sessile, yellow (!Hatschbach). Ovary slenderly fusiform, barely distinct, ca. $10 \mathrm{~mm}$ long. Perianth-tube slenderly subcylindric, $22 \mathrm{~mm}$ long, $2 \mathrm{~mm}$ wide, laxly vestite with minute pointed trichomes. Tepals oblong, broadly rounded, $11 \mathrm{~mm}$ long. Anthers 7 $\mathrm{mm}$ long, extending downward into the perianthtube; coronoid appendages exceeding the anthers, suboblong with narrowly triangular obtuse lobes. Style about equaling the anthers; stigmas not observed.

LeAF Anatomy (Hatschbach 30095).-Surface View: Hairs: none observed. Epidermis: cells on both surfaces square to rectangular; walls thickened. Stomata: paracytic and tetracytic; $24 \times 18 \mu \mathrm{m}$; present on abaxial surface.

Transverse Section of Lamina: Dorsiventral; widely V-shaped with median adaxial groove. Surface only gently undulating. Epidermis: cells on both surfaces rounded to dome-shaped; few conical; walls thickened. One or two layers of rounded, thin-walled cells subjacent to adaxial epidermis; larger cells above midvein. Cuticle: thickened and ridged on abaxial surface; very thick and ridged on adaxial surface. Stomata: present on abaxial surface only; stomata flush with epidermal surface or slightly sunken; substomatal chamber present. Mesophyll: 3 or 4 layers of palisade cells followed by 6 or 7 layers of small, rounded, thin-walled cells. Vascular bundles: 15; commissural bundles observed. Two or three large vessels present in each bundle. Two phloem units lying laterally in flanges of $\mathrm{Y}$-shaped abaxial girder. Long and slender inverted $\mathrm{Y}$-shaped adaxial girder present on each bundle. Each vascular bundle completely surrounded by a bundle sheath. Crystals: druses present in mesophyll. Tannins: present.

TYPE.-On wet cliffs by small waterfall, road to Pico de Itambé, Mun. Santo Antonio de Itambé, Minas Gerais, Brazil, 1972, Hatschbach 27513 (US, holotype; MBM, isotype).

Distribution.-Brazil: Minas Gerais: Typelocality, 9 August 1972, Hatschbach 30095 (MBM, US).

Note: Dr. P. F. Ravenna has kindly allowed us to publish his new species in our revision in order to relate it to others more immediately.

\section{Barbacenia sessiliflora L. B. Smith}

Barbacenia sessiliflora L. B. Smith, Contr. U.S. Nat. Herb., 35:291, pl. 12: figs. 66-68, 1962.

TyPe--On rock, Serra Cata Altas, Mun. Santa Barbara, Minas Gerais, Brazil, 2 February 1943, Magalhães 6400 (NY, holotype).

Distribution.-Known only from the typecollection.

68. Barbacenia nana L. B. Smith \& Ayensu, new species

Plates 19, 34e, $f$

A B. gentianode Goethart \& Henrard, cui valde affinis, foliorum laminis floribusque subdense albopubescentibus differt.

Caudex simple, to $8 \mathrm{~cm}$ long, ca. $2 \mathrm{~cm}$ thick with old leaf-bases. Leaves sulcate throughout; sheaths completely covered; blades $8 \mathrm{~cm}$ long, $9 \mathrm{~mm}$ wide, subdensely white-pubescent throughout, very finely ciliate with some hairs slightly enlarged at apex. 
Scapes not observed. Flowers solitary, appearing sessile, subdensely white-pubescent, red. Ovary slenderly ellipsoid, ca. $8 \mathrm{~mm}$ long, scarcely distinct. Perianth-tube (whole) slenderly cylindric, $45 \mathrm{~mm}$ long, $3 \mathrm{~mm}$ wide. Tepals narrowly elliptic, obtuse, $20 \mathrm{~mm}$ long, $4 \mathrm{~mm}$ wide. Anthers exceeding the appendages by $9 \mathrm{~mm}$; coronoid appendages united into a tube for most of their length, their free apices narrowly triangular with minute rounded lobes. Style much exceeding the anthers, ellipsoid at apex with 3 narrow apically confluent stigmas.

Leaf Anatomy.-Ayensu, Smithsonian Contr. Bot., 15:65, fig. 2c, pl. 8c,d, 22b, 47f, 1974.

TYPE.-On rock outcrop by Rio Santo Antônio, Mun. Conceição do Mato Dentro, 18 January 1972, L. B. Smith, Ayensu \& Hatschbach (separate no. $28894) 15973$ (US, holotype; MBM, isotype).

Distribution.-Known only from the typecollection.

\section{Barbacenia gentianoides Goethart \& Henrard}

Barbacenia gentianoides Goethart \& Henrard, Blumea, 2:347, fig. M, 1937.-L. B. Smith, Contr. U.S. Nat. Herb., 35:280, 1962.-Ayensu, Smithsonian Contr. Bot., 15:64, fig. 20a-c, $41 \mathrm{c}-\mathrm{e}, 1974$.

\section{9a. Barbacenia gentianoides var. gentianoides}

Aylthonia gentianoides (Goethart \& Henrard) Menezes, Ciência e Cultura, 23(3):[421], 1971.

Leaves shorter than the flowers, densely whitepubescent beneath; flowers to $9 \mathrm{~cm}$ long, fire red.

TYPE.- "Rio dos Pedros" (Rio das Pedras), Valu, Minas Gerais, Brazil, 2 May 1892, Glaziou 19922 (L, holotype; B, photo s n, 144, 145).

Distribution.-Brazil: Minas Gerais: Diamantina, Datas, Jaboticatubas, Conceição do Mato Dentro.

69b. Barbacenia gentianoides var. magalhaesii

(L. B. Smith) L. B. Smith \& Ayensu, new combination, new status

Barbacenia magalhaesii L. B. Smith, Contr. U.S. Nat. Herb. 35:291, pl. 12: figs. 63-65, 1962.

Aylthonia magalhaesii (L. B. Smith) Menezes, Ciência e Cultura, 23(3):[421], 1971.

Leaves much exceeding the flowers; blades glabrous beneath.
TYPE.-On sandstone rocks and ledges, road from Hotel Chapeu de Sol, Serra Cipó to Conceição, km 120-140, Minas Gerais, Brazil, 1200-1300 m alt, 8 August 1960, Maguire, Magalhães \& Maguire 49094 (US, holotype; NY, isotype).

Distribution.-Brazil: Minas Gerais: Conceição do Mato Dentro.

\section{Barbacenia plantaginea L. B. Smith}

Barbacenia plantaginea L. B. Smith, Phytologia, 8:513, fig. 20, 22, 1963.

TyPE.-Serra da Lapa (Serra do Cipó), Minas Gerais, Brazil, December 1824, Riedel 1061 (K, holotype).

Distribution.-Brazil: Minas Gerais: Datas, Conceição do Mato Dentro.

\section{Barbacenia hilairei Goethart \& Henrard}

Barbacenia hilairei Goethart \& Henrard, Blumea, 2:351, 1937. L. B. Smith, Contr. U.S. Nat. Herb., 35:280, 1962.

Type.-Minas Gerais, Brazil, St.-Hilaire $B^{1} 635$ (P, holotype; B, photo 119).

Distribution.-Known only from the typecollection.

\section{Barbacenia oxytepala Goethart \& Henrard}

Barbacenia oxytepala Goethart \& Henrard, Blumea, 2:357, 1937.-L. B. Smith, Contr. U.S. Nat. Herb., 35:280, 1962.

TyPE.-Rare on wet rocks, Biribiri, Mun. Diamantina, Minas Gerais, Brazil, March 1892, Schwacke 8353 (B, holotype; B, photo 183).

Distribution.-Known only from the typecollection.

\section{Barbacenia grisea $\mathbf{L}$. B. Smith}

Barbacenia grisea L. B. Smith, Contr. U.S. Nat. Herb., 35:280, pl. 8, 1962.

Type-Ca. 49 miles from Diamantina, Minas Gerais, Brazil, $1140 \mathrm{~m}$ alt, 22 December 1959, $M a$ guire, Maguire of Pires 44745 (NY, holotype).

Distribution.-Known only from the typecollection. 
74. Barbacenia filamentifera L. B. Smith \& Ayensu, new species

\section{Plates 20, 35a,b}

A B. grisea L. B. Smith, cui verisimiliter affinis, foliis anguistioribus, tubo perianthii valde curvato dense brunneo-piloso differt.

Caudex not seen. Leaf-blades linear, filiformattenuate, $13 \mathrm{~cm}$ long, $7 \mathrm{~mm}$ wide, sulcate on both sides, laxly setose-ciliate, otherwise even and glabrous.

Scape $13 \mathrm{~cm}$ long, densely brown-pilose. Flower red (!Hatschbach \& Pelanda), densely brown-pilose. Ovary slenderly ellipsoid, indistinct, ca. $15 \mathrm{~mm}$ long. Perianth-tube (whole) slenderly subcylindric, strongly curved, $5 \mathrm{~cm}$ long, $7 \mathrm{~mm}$ wide. Tepals nearly uniform, narrowly lanceolate, acute and apiculate, $25 \mathrm{~mm}$ long. Anthers unknown; coronoid appendages (incomplete) suboblong, over $5 \mathrm{~mm}$ long. Style and stigmas unknown.

Leaf Anatomy (Hatschbach \& Pelanda 27955).Surface View: Hairs: none observed. Epidermis: cells on both surfaces 4- to 6-sided, but mostly rectangular; thin-walled. Stomata: tetracytic; $24 \times 18$ $\mu \mathrm{m}$; present in rows on both surfaces.

Transverse Section of Lamina: Dorsiventral; $V$-shaped with margins turned slightly downward. Both surfaces very gently undulating. Epidermis: cells on both surfaces rounded to dome-shaped; few conical; thin-walled. Cuticle: slightly thickened and smooth over entire surface. Stomata: present on both surfaces; stomata flush with epidermal surface; small substomatal chamber present. Mesophyll: 3 layers of small palisade cells followed by 4 or 5 layers of large, rounded, thin-walled, translucent cells. Subjacent to these occur 4 or 5 layers of smaller, rounded cells; some translucent. Large, translucent cells above midvein. Vascular bundles: 29; commissural bundles observed. One large vessel in each bundle. Two phloem units present in flanges of thick Y-shaped abaxial girder which extends to abaxial epidermis. Thick, inverted $Y$-shaped adaxial girder present; extending to adaxial epidermis and laterally along epidermis. Bundle sheath completely surrounding each vascular bundle. Crystals: druses present in many mesophyll cells. Tannins: present in mesophyll and epidermis.

TYPE.-On rock outcrops by Route BR 259, Mun. Datas, Minas Gerais, Brazil, $1400 \mathrm{~m}$ alt, 14
November 1971, Hatschbach \& Pelanda 27955 (US, holotype; MBM, isotype).

Distribution.-Known only from the typecollection.

Note: It is hoped that the above unsatisfactory description can be improved by further collections since the type-locality is so well defined.

\section{Barbacenia pallida L. B. Smith \& Ayensu, new species}

Plates 21, 35c,d

A B. flamentifera L. B. Smith \& Ayensu, cui affinis, foliorum laminis aequaliter attenuatis vix filamentiferis scapum superantibus, floribus albis, tubo perianthii recto laxe piloso differt.

Caudex $20 \mathrm{~cm}$ high (!Irwin. "Herb to ca. $20 \mathrm{~cm}$. tall."), over $12 \mathrm{~mm}$ thick including leaf-bases. Leaves 3-ranked, sulcate on both sides; sheaths slightly exposed at apex; blades linear, evenly attenuate, $22 \mathrm{~cm}$ long, $5 \mathrm{~mm}$ wide at base, ciliate with erect or suberect setae, otherwise glabrous.

Scapes solitary, $15 \mathrm{~cm}$ long above the leaf-sheaths, laxly pale-pilose. Flowers white (!Irwin et al.), laxly pale-pilose. Ovary slenderly ellipsoid, slightly wider than the upper perianth-tube, ca. $20 \mathrm{~mm}$ long. Perianth-tube (whole) 45-60 mm long, slenderly cylindric above the ovary, 4-5 $\mathrm{mm}$ wide. Tepals nearly uniform, narrowly oblong, 25-30 mm long, the inner nearly glabrous. Anthers erect, $13 \mathrm{~mm}$ long, attached near their base and near the apex of the appendage; coronoid appendages subtriangular, obtuse, unlobed, $12 \mathrm{~mm}$ long. Style exceeding the anthers, ellipsoid-enlarged at apex with 3 oblong apically confluent stigmas.

Leaf ANatomy (Irwin et al. 22608).-Surface View: Hairs: few tufts of hairs on edge of lamina. Epidermis: cells on adaxial surface square to rectangular; walls slightly thickened. Abaxial cells usually square to rectangular; few with more sides; walls slightly thickened. Stomata: paracytic and tetracytic; $30 \times 18 \mu \mathrm{m}$; present on abaxial surface in rows.

Transverse Section of Lamina: Dorsiventral; widely V-shaped. Both surfaces only very gently undulating. Epidermis: cells rounded to domeshaped; walls slightly thickened; outer tangential walls thicker. Cuticle: thin and smooth over entire surface. Stomata: present on abaxial surface only; 
outer ledges present; stomata flush with epidermal surface; small substomatal chamber present. Mesophyll: 4 layers of small palisade cells followed by 3 layers of large, rounded, thin-walled cells, mostly translucent. Below this occur 3 or 4 layers of compactly arranged rounded cells. Translucent cells occur above midvein. Vascular bundles: 27; commissural bundles observed. One or two large vessels present in each bundle, mostly one. Two phloem units lying laterally in flanges of $Y$-shaped abaxial girder. Inverted $Y$-shaped adaxial girder present on each bundle. Girders extend to epidermis. Bundle sheath completely surrounding each vascular bundle. Crystals: druses in all of mesophyll except translucent cells. Tannins: present.

TYPE.-Rocky cerrado, ca. $15 \mathrm{~km}$ northeast of Diamantina on road to Mendanha, Minas Gerais, Brazil, $1300 \mathrm{~m}$ alt, 26 January 1969, Irwin, Reis dos Santos, Souza \& Fonseca 22608 (NY, holotype; US, photo).

Distribution.-Known only from the typecollection.

Note: The flower of $B$. pallida strongly resembles that of $B$. gentianoides Goethart \& Henrard, but its coronoid appendages are free and unlobed.

\section{Barbacenia salmonea L. B. Smith \& Ayensu, new species}

Plates 22, 35e,f

A B. pallida L. B. Smith \& Ayensu, cui affinis, tepalis antherisque recurvatis, floribus salmoneis, appendicibus coronoideis multo minoribus differt.

Caudex (incomplete) over $4 \mathrm{~cm}$ long, $15 \mathrm{~mm}$ thick including leaf-sheaths. Leaves obscurely 3ranked; sheaths covered; blades linear, evenly attenuate, $12 \mathrm{~cm}$ long, $4 \mathrm{~mm}$ wide, appearing resinous-spotted beneath when dry, laxly setoseciliate, otherwise glabrous.

Scape single, $9 \mathrm{~cm}$ long above the leaf-sheaths, laxly pale-pilose. Flowers salmon (!Sazima, Sazima \& Menezes), very laxly pale-pilose. Ovary slenderly turbinate, ca. $20 \mathrm{~mm}$ long, barely distinct. Perianthtube (whole) slenderly cylindric, $55 \mathrm{~mm}$ long, $4 \mathrm{~mm}$ wide. Tepals recurving, nearly uniform, oblong, rounded and apiculate, $17 \mathrm{~mm}$ long, $4 \mathrm{~mm}$ wide. Anthers recurving, $11 \mathrm{~mm}$ long, apiculate; coronoid appendices subelliptic with 2 short subacute lobes, $3 \mathrm{~mm}$ long. Style ellipsoid at apex with 3 broad apically confluent stigmas.
Leaf Anatomy. (Sazima, Sazima \& Menezes 239). -Surface View: Hairs: none observed. Epidermis: cells square to rectangular on both surfaces; few irregular among stomata; walls thickened. Stomata: tetracytic; $24 \times 18 \mu \mathrm{m}$; present in rows on abaxial surface.

Transverse Section of Lamina: Dorsiventral; widely V-shaped with extreme margin tips turned downward. Both surfaces slightly undulating. Epidermis: rounded to dome-shaped on both surfaces; walls thickened. One layer of rounded parenchyma cells subjacent to adaxial epidermis. Larger cells above midvein. Cuticle: thin and smooth on entire surface. Stomata: present on abaxial surface only; stomata flush with epidermal surface; small substomatal chamber present. Mesophyll: 3 layers of small palisade cells followed by 4 layers of large, rounded, thin-walled cells. Below this occur 3 or 4 layers of small, compactly arranged, rounded cells. Some mesophyll cells appear translucent. Vascular bundles: 27; commissural bundles observed. One large vessel present in each bundle. Two phloem units lying laterally in flanges of $Y$-shaped abaxial girder. Slightly slender inverted $Y$-shaped adaxial girder present on each bundle. Bundle sheath completely surrounding each vascular bundle. Crystals: present in most of mesophyll. Tannins: present.

TYPE.-On rocks by brook, high ground, km 126 on the road from Lagoa Santa to Conceição do Mato Dentro, Serra do Cipó, Minas Gerais, Brazil, 23 July 1972, Sazima, Sazima \& Menezes 239 (US, holotype; $\mathrm{SPF}$, isotype).

Distribution.-Known only from the typecollection.

\section{Barbacenia glabra Goethart \& Henrard}

Barbacenia glabra Goethart \& Henrard, Blumea, 2:348, fig. H, 1937.-L. B. Smith, Contr. U.S. Nat. Herb., 35:281, 1962.

Trpe.-Minas Gerais, Brazil, St.-Hilaire $C^{\boldsymbol{1}} 352$ (P, holotype; B photo 137).

Distribution.-Known only from the typecollection.

78. Barbacenia nuda L. B. Smith \& Ayensu, new species Plates 23, 36a, $b$

A B. glabra Goethart \& Henrard, cui affinis, foliis 
latioribus quam scapo brevioribus, tepalis obtusis, appendicibus coronoideis apice valde dilatatis cum lobis suborbicularibus differt.

Caudex (incomplete) over $2 \mathrm{~cm}$ long, completely covered by leaves. Leaf-blades linear, attenuate, 8 cm long, $8 \mathrm{~mm}$ wide, sulcate on both sides, serrulate on margins and keel especially toward apex, otherwise smooth and glabrous.

Scapes 2, $9 \mathrm{~cm}$ long, glabrous. Flower dull red when dry, glabrous. Ovary slenderly fusiform, indistinct, ca. $20 \mathrm{~mm}$ long. Perianth-tube (whole) slenderly cylindric, $45 \mathrm{~mm}$ long, $4 \mathrm{~mm}$ wide. Tepals $20 \mathrm{~mm}$ long, obtuse, the outer lanceolate and $4 \mathrm{~mm}$ wide, the inner narrowly ovate and $6 \mathrm{~mm}$ wide. Anthers $7 \mathrm{~mm}$ long; coronoid appendages spatulate with 2 suborbicular lobes, $6 \mathrm{~mm}$ long. Style ellipsoid at apex with 3 broad apically confluent stigmas.

Leaf Anatomy (Semir \& Menezes 282).-Surface View: Hairs: none observed. Epidermis: cells on both surfaces square to rectangular; thin-walled. Stomata: tetracytic; $24 \times 18 \mu \mathrm{m}$; present in rows on both surfaces.

Transverse Section of Lamina: Dorsiventral; deeply V-shaped. Both surfaces quite smooth. Epidermis: cells on both surfaces rounded to domeshaped; thin-walled. Cuticle: thick and ridged on entire surface. Stomata: present on both surfaces; stomata flush with epidermal surface; substomatal chamber present. Mesophyll: 4 or 5 layers of small palisade cells followed by 4 or 5 layers of rounded, thin-walled cells. Vascular bundles: 59; commissural bundles observed. One large vessel present in each bundle. Two phloem units lying laterally in flanges of Y-shaped abaxial girder. Inverted Y-shaped adaxial girder present on each bundle. Girders extending to epidermis on both sides. Each vascular bundle completely surrounded by a conspicuous bundle sheath. Crystals: none observed. Tannins: present, especially in epidermis.

TyPE.-Near brook and waterfall, $\mathrm{km} 126$ of the road from Lagoa Santa to Conceição do Mato Dentro, Serra do Cipó, Minas Gerais, Brazil, 2 November 1972, (Semir \&) Menezes 282 (US, holotype; SPF, isotype).

Distribution.-Known only from the typecollection.

\section{Barbacenia tomentosa Martius}

Barbacenia tomentosa Martius, Nov. Gen. \& Sp., 1:18, pl. 11,
1823.-Seubert in Martius, Fl. Bras., 3(1):69, 1847.-L. B. Smith, Contr. U.S. Nat. Herb., 35:281, 1962.-Ayensu, Smithsonian Contr. Bot,, 15:67, pl. 7c,d, 1974.

Aylthonia tomentosa (Martius) Menezes, Ciência e Cultura, 23(3):422, 1971.

TYPE.-On rocks of micaceous granular schist, between São João del Rei and Villa Rica, (Ouro Preto), and in Diamantina, Minas Gerais, Brazil, Martius s $n$ (M, holotype $\mathrm{n} \mathrm{v}$ ). In the absence of a specimen well typified by the original description and illustration.

Distribution.-Brazil: Minas Gerais: on rocks, lower slopes of Pico de Itacolumí, $3 \mathrm{~km}$ south of Ouro Preto, $1650 \mathrm{~m}$ alt, 1 February 1971, Irwin, Harley \& Omishi 29559 (NY, UB, US).

\section{Barbacenia cyananthera L. B. Smith \& Ayensu, new species}

Plates 24, 36c,d

A B. tomentosa Martius, cui affinis, foliorum laminis glabris, tepalis subduplo majoribus, appendicibus coronoideis valde majoribus differt.

Caudex very short. Leaves subrosulate; sheaths covered; blades linear, evenly attenuate, $12 \mathrm{~cm}$ long, $6 \mathrm{~mm}$ wide, sulcate on both sides, setose-ciliate, otherwise even and glabrous.

Scapes solitary, 4-5 cm long above the leafsheaths, laxly pale-pubescent. Flowers scarlet (IIrwin et al.), laxly pale-pubescent. Ovary turbinate, indistinct, ca. $12 \mathrm{~mm}$ long. Perianth-tube (whole) slenderly cylindric, $30 \mathrm{~mm}$ long, $5 \mathrm{~mm}$ wide. Tepals uniform, oblong, obtuse, $20 \mathrm{~mm}$ long. Anthers ca. $6 \mathrm{~mm}$ long, attached near their base to near apex of appendage; coronoid appendage subtriangular, acutely bilobed, ca. $6 \mathrm{~mm}$ long. Style much exceeding stamens, ellipsoid-enlarged at apex and almost wholly covered by the confluent stigmas.

LeAF АNATOMY (Irwin, Maxwell \& Wasshausen 19834).- Surface View: Hairs: few tufts observed. Epidermis: cells on both surfaces 4- to 6-sided; thinwalled. Stomata: tetracytic; $24 \times 18 \mu \mathrm{m}$; present in rows on both surfaces.

Transverse Section of Lamina: Dorsiventral; very widely V-shaped. Few tufts of hairs present on both surfaces and at extreme margins. Epidermis: cells on both surfaces small; rounded to dome-shaped; few conical; thin-walled. Cuticle: thin and smooth on most of surface; thicker above and/or below few 
bundles. Stomata: present on both surfaces; stomata flush with epidermal surface; small substomatal chamber present. Mesophyll: 3 or 4 layers of small palisade cells followed by 3 or 4 layers of large, rounded, translucent cells. Below this occur 4 or 5 layers of small, rounded, compactly arranged spongy tissue. Vascular bundles: 19; commissural bundles observed. One or two large vessels present in each bundle, mostly one. Two phloem units lying laterally in flanges of thick, Y-shaped abaxial girder which extends to the abaxial epidermis. Relatively thin, inverted $\mathrm{Y}$-shaped adaxial girder present on each bundle, extending to adaxial epidermis. Each vascular bundle completely surrounded by a bundle sheath. Crystals: few present in mesophyll. Tannins: present.

Typk.-Extensive mats on rocks, steep slopes, Pico de Itabirito, ca. $50 \mathrm{~km}$ southeast of Belo Horizonte, Minas Gerais, Brazil, $1750 \mathrm{~m}$ alt, 11 February 1968, Irwin, Maxwell \& Wasshausen 19834 (NY, holotype; UB; US, isotypes).

Distribution.-Known only from the typecollection.

\section{Barbacenia schidigera Lemaire}

Barbacenia schidigera Lemaire, Jard. F1., 2, pl. 198, 1852.L. B. Smith, Contr. U.S. Nat. Herb., 35:281, 1962.

TYPE.-On rocks above a cascade, peak of Itacolumí, Minas Gerais, Brazil, 1849, Libon s $n$ (?), cultivated November 1851, De Jonghe s $n$ (BR(?) $\mathrm{n} v$ ). In the absence of any specimen the species is well typified by the original description and illustration.

Distribution.-Known only from the typecollection.

\section{Barbacenia graciliflora L. B. Smith}

Barbacenia graciliflora L. B. Smith, Contr. U.S. Nat. Herb., 35:281, pl. 10: figs. 27, 28, 1962.

TYPE.-On rocks at edge of stream, sandstone area, Serra de Monjolo, $18 \mathrm{~km}$ north of Sêrro, Mun. Sêrro, Minas Gerais, Brazil, 5 May 1945, Williams \& Assis 6816 (GH, holotype).

Distribution.-Known only from the typecollection.

\section{Barbacenia pulverulenta L. B. Smith \& Ayensu, new species}

Plates 25, 36e, $f$

A B. ensifolia Martius ex Schultes f., cui verisimiliter affinis, foliis scapum multo superantibus, perianthii glandulis minimis pallidis differt.

Caudex over $6 \mathrm{~cm}$ long, completely covered by reflexed leaves. Leaf-sheaths wholly covered; blades linear, filiform-attenuate, $13 \mathrm{~cm}$ long, $5 \mathrm{~mm}$ wide, sulcate on both sides, grayish green from a dense coat of vitreous glands ca. $0.05 \mathrm{~mm}$ long.

Scape $4 \mathrm{~cm}$ long, microscopically pale-glandular. Flower vestite like scape, orange (!Menezes). Ovary slenderly turbinate, ca. $25 \mathrm{~mm}$ long, barely distinct. Perianth-tube (whole) slenderly subcylindric, $55 \mathrm{~mm}$ long, $3 \mathrm{~mm}$ wide. Tepals oblong, contracted toward base, rounded and apiculate at apex, $15 \mathrm{~mm}$ long, $2.5 \mathrm{~mm}$ wide. Anthers not seen; coronoid appendages (incomplete) subtriangular, $5 \mathrm{~mm}$ long without lobes. Style exceeding the tepals, the apex ellipsoid and completely covered by the stigmas.

Leaf Anatomy (Menezes 245).-Surface View: Hairs: small hairs present on abaxial surface. Epidermis: cells on both surfaces square to rectangular; walls slightly thickened. Stomata: paracytic and tetracytic; $18 \times 15 \mu \mathrm{m}$; present in abaxial furrows only.

Transverse Section of Lamina: Dorsiventral; almost horizontal. Adaxial surface ridged; abaxial surface furrowed about $1 / 2$ thickness of blade. Small hairs present on abaxial surface. Epidermis: cells rounded to dome-shaped; walls slightly thickened; cells larger above midvein. Cuticle: thick and ridged on both surfaces. Stomata: present in abaxial furrows only; stomata raised above the surface of epidermis and protected by two large, protruding subsidiary cells on either side; fairly large substomatal chamber present. Mesophyll: in area not above furrows occur one or two layers of slightly elongated palisade cells followed by 7 layers of rounded, compactly arranged cells. Above the abaxial furrows are 2 or 3 layers of thick-walled cells followed by 3 layers of rounded, compactly arranged cells. Two layers of cells above midvein elongated and thick-walled. Vascular bundles: 23; commissural bundles observed. One or two large vessels present in each bundle, mostly one. Two phloem units lying laterally in flanges of very short 
Y-shaped abaxial girder; girder extends laterally along the abaxial epidermis with the tips curving adaxially. Adaxial girder is T-shaped becoming wider near the epidermis and extending laterally along it. Lateral extensions of adaxial girder are continuous with thick-walled cells above the furrows. No adaxial girder on midvein. Bundle sheath completely surrounding each vascular bundle. Crystals: none observed. Tannins: many present.

Note: This species is characterized by the extensive development of abaxial sclerenchyma girder and the distinctly shaped furrows.

Type.-Near kaolin mine, $\mathrm{km} \mathrm{114}$, to the left (west) of the road from Lagoa Santa to Conceição do Mato Dentro, Serra do Cipó, Minas Gerais, Brazil, 24 July 1972, Menezes 245 (US, holotype; SPF, isotype).

Distribution.-Known only from the typecollection.

\section{Barbacenia ensifolia Martius ex Schultes f.}

Barbacenia ensifolia Martius ex Schultes f. in Roemer \& Schultes, Syst., 7:287, 1826.-Seubert in Martius, Fl. Bras., 3(1):71, 1847.-L. B. Smith, Contr. U.S. Nat. Herb., 35:282, 1962.

Type.-Serra de Lapa (Serra do Cipó), Minas Gerais, Brazil, Martius s $n$ (M, holotype; F, photo 18990).

Distribution.-Known only from the typecollection.

\section{Barbacenia glaziovii Goethart \& Henrard}

Barbacenia glaziovii Goethart \& Henrard, Blumea, 2:348, 1937.-L. B. Smith, Contr. U.S. Nat. Herb., 35:282, 1962.

TYPE.-Among rocks, Biribiri, Mun. Diamantina, Minas Gerais, Brazil, 23 March 1892, Glaziou 19930 (L, holotype; B, photo s n, 134).

Distribution.-Brazil: Minas Gerais: in sandy soil of brookbank, campo zone, Biribiri, 15 November 1971, Hatschbach \& Pelanda 27978 (MBM, US).

\section{Barbacenia conicostigma Goethart \& Henrard}

Barbacenia conicostigma Goethart \& Henrard, Blumea, 2:341, 1937.-L. B. Smith, Contr. U.S. Nat. Herb., 35:282, 1962.Ayensu, Smithsonian Contr. Bot., 15:63, fig. 1g,h, pl. 23a,b, $46 \mathrm{e}, 1974$.
TyPE.-On rocks of high peak, Serra da Lapa (Serra do Cipó), Minas Gerais, Brazil, 1824, Riedel $s n$ (LE, holotype; B, photo 115).

Distribution.-Brazil: Minas Gerais: Serra do Cipó, Datas, Gouvêa, Jaboticatubas.

\section{Barbacenia cuspidata Goethart \& Henrard}

Barbacenia cuspidata Goethart \& Henrard, Blumea, 2:342, fig. C, 1937.-L. B. Smith, Contr. U.S. Nat. Herb., 35:282, 1962.

Aylthonia cuspidata (Goethart \& Henrard) Menezes, Ciência e Cultura, 23(3):[421], 1971.

TyPE.-Minas Gerais(?), Brazil, Glaziou 19927 e p (P, holotype; $\mathrm{B}$, photo 135$)$.

Distribution.-Known only from the typecollection.

\section{Barbacenia albiflora L. B. Smith}

Barbacenia albiflora L. B. Smith, Contr. U.S. Nat. Herb., $35: 292$, pl. 12: figs. $69,70,1962$.

TYPE.-On sandstone, Serra Grão Mogul, north base of mountain, Minas Gerais, Brazil, 600-700 $\mathrm{m}$ alt, Maguire, Magalhães \& Maguire 49213 (US, holotype; NY, isotype).

Distribution.-Known only from the typecollection.

89. Barbacenia bibiriensis L. B. Smith \& Ayensu, new species

\section{Plates 26, 37a,b}

A B. albiflora L. B. Smith, cui affinis, floribus viridibus, tepalis erectis differt.

Caudex unknown. Leaf-blades very narrowly triangular, filiform-attenuate, $28 \mathrm{~cm}$ long, $9 \mathrm{~mm}$ wide, laxly vestite with minute white pustulate-based gland-tipped hairs, ciliate with similar hairs up to $5 \mathrm{~mm}$ long.

Scapes (complete) to $38 \mathrm{~cm}$ long, laxly vestite with minute pale stipitate glands and much larger black ones. Flowers glandular like the scapes but more laxly so, green outside, white inside (!Hatschbach). Ovary ellipsoid distinct, $15 \mathrm{~mm}$ long. Perianth-tube above ovary slenderly subcylindric flaring slightly at apex, $32 \mathrm{~mm}$ long, $3 \mathrm{~mm}$ wide at base. Tepals narrowly ovate, obtuse, $20 \mathrm{~mm}$ long, the inner broader and nearly 
glabrous. Anthers $11 \mathrm{~mm}$ long, attached submedianly to the appendage and extending below it; coronoid appendage suboblong, $5 \mathrm{~mm}$ long including the 2 acute lobes. Style exceeded by the stamens, its apex subconical with 3 linear apically confluent stigmas.

LEAF ANATOMY (Hatschbach 30190).- Surface View: Hairs: multicellular, tufts of hairs present on both surfaces. Epidermis: cells on both surfaces square to rectangular; thin-walled. Stomata: tetracytic; $21 \times 18 \mu \mathrm{m}$; present on both surfaces.

Transverse Section of Lamina: Dorsiventral; widely V-shaped. Multicellular tufts of hairs present on both surfaces. Surface slightly undulating. Epidermis: cells on both surfaces rounded to domeshaped; few conical; thin-walled. Cuticle: slightly thickened and smooth over entire surface. Stomata: present on both surfaces; substomatal chamber present; stomata flush with epidermal surface. Mesophyll: 1 or 2 layers of slightly elongated palisade cells followed by 4 or 5 layers of rounded, closely packed cells; few with thickened walls. Rounded, translucent cells above midvein. Vascular bundles: 33; commissural bundles observed. One or two large vessels present in each bundle; mostly one. Two phloem units lying laterally in flanges of short Y-shaped abaxial girder which extends to abaxial epidermis. Short, inverted, $Y$-shaped adaxial girder present on each bundle and extends to adaxial epidermis. Bundle sheath surrounding each vascular bundle. Crystals: druses and styloids present in most of mesophyll. Tannins: large quantity in mesophyll especially toward midrib.

TYPE.-On rock outcrops, Biribiri, Mun. Diamantina, Minas Gerais, Brazil, 11 August 1972, Hatschbach 30190 (US, holotype; MBM, isotype).

Distribution.-Known only from the typecollection.

Note: Name derived from "bi" (twice) and "biri."

\section{Barbacenia longiflora Martius}

Barbacenia longiflora Martius, Nov. Gen. \& Sp., 1:19, pl. 12, 1823.-Seubert in Martius, Fl. Bras., 3(1):70, 1847.-L. B. Smith, Contr. U.S. Nat. Herb., 35:282, 1962.-Ayensu, Smithsonian Contr. Bot., 15:64, fig. 24d-f, pl. 41f-h, 1974.

Type.-On grassy banks, Tejuco (Diamantina), Martius s n (M, holotype; F, photo 18995).
Distribution.-Brazil: Minas Gerais: Diamantina, São João da Chapada.

\section{Barbacenia gardneri Seubert}

Barbacenia gardneri Seubert in Martius, Fl. Bras., 3(1):70, pl. 8: fig. 5, 1847.-L. B. Smith, Contr. U.S. Nat. Herb., 35:282, 1962.-Ayensu, Smithsonian Contr. Bot., 15:64, pls. 7a,b, $47 \mathrm{a}, 1974$.

TYPE.-Minas Gerais, Brazil, Gardner 5217 (M, holotype(?) n v; W, isotype; F, photo 30021).

Distribution.-Known only from the typecollection.

\section{Barbacenia curviflora Goethart \& Henrard}

Barbacenia curviflora Goethart \& Henrard, Blumea, 2:342, 1937.-L. B. Smith, Contr. U.S. Nat. Herb., 35:282, 1962.

TYPE,-On rocky ground, "Rio dos Pedros" (Rio das Pedras), Valu, Minas Gerais, Brazil, 2 May [1892], Glaziou 19923 (B, holotype; B, photo 123).

Distribution.--Known only from the typecollection.

\section{Barbacenia fragrans Goethart \& Henrard}

Barbacenia fragrans Goethart \& Henrard, Blumea, 2:345, 1937.-L. B. Smith, Contr. U.S. Nat. Herb., 35:282, 1962.Ayensu, Smithsonian Contr. Bot., 15:63, figs. 2d, 24a-c, pls. $9 \mathrm{a}, 22 \mathrm{a}, 1974$.

TYPE.-On dry rocks, sometimes in moist cracks in rocks, Caldas, Minas Gerais, Brazil, 1 February 1876, Mosén 4444 (S, holotype).

Distribution.-Brazil: Minas Gerais: Caldas. São Paulo: São João da Boa Vista.

\section{Barbacenia tricolor Martius}

Barbacenia tricolor Martius, Nov. Gen. \& Sp., 1:18, pl. 10, 1823.-Seubert in Martius, F1. Bras., 3(1):70, 1847.-L. B. Smith, Contr. U.S. Nat. Herb., 35:282, 1962.

Aylthonia tricolor (Martius) Menezes, Ciência e Cultura, 23(3):422, 1971 .

TYPE.-In ferruginous and micaceous mountains, Minas Gerais, Brazil, Martius s n (M, holotype(?) $\mathrm{n}$ v).

Distribution.-Brazil: Minas Gerais: São Tomé das Letras, $21^{\circ} 43^{\prime} \mathrm{S}, 44^{\circ} 59^{\prime} \mathrm{W}$, recent collection near Martius' route and possible topotype (Menezes 115). 


\section{Barbacenia blanchetii Goethart \& Henrard}

Barbacenia blanchetii Goethart \& Henrard, Blumea, 2:339, 1937.-L. B. Smith, Contr. U.S. Nat. Herb., 35:282, 1962.

Type.-Bahia, Brazil, Blanchet 3278 (B, holotype; $\mathrm{W}$, isotype; $\mathrm{F}$, photo 30020 ).

Distribution.-Brazil: Bahia: "Igreja Vilha," Blanchet 3273 (G, !Henrard). Jacobina, Blanchet 2537 (G, !Henrard). "Ponço d'Areia" [Pouço d'Areia], Blanchet 3870 (G, P, !Henrard). In fissures in sandstone above $100 \mathrm{~m}$ falls of Rio Ferro Doide, ca. $18 \mathrm{~km}$ east of Morro do Chapéu, $300 \mathrm{~m}$ alt, 20 February 1971, Irwin, Harley of Smith 30691 (NY, UB, US), near Jacobina and possibly topotypic. Andarai, Mucugê.

\section{Barbacenia mollis Goethart \& Henrard}

Barbacenia mollis Goethart \& Henrard, Blumea, 2:356, 1937. -L. B. Smith, Contr. U.S. Nat. Herb., 35:282, 1962.

\section{6a. Barbacenia mollis var. mollis}

Leaf-blades to $12 \mathrm{~cm}$ long, $12 \mathrm{~mm}$ wide.

Type.-Minas Gerais, Brazil, St.-Hilaire C 962 (P, holotype, $\mathrm{n}$ v).

Distribution.-Known only from the typecollection.

\section{6b. Barbacenia mollis var. microphylla L. B. Smith}

Barbacenia mollis Goethart \& Henrard var. microphylla L. B. Smith, Contr. U.S. Nat. Herb., 35:282, 1962.

Leaf-blades to $8 \mathrm{~cm}$ long, $4 \mathrm{~mm}$ wide. Flower red (!Williams \& Assis).

Type.-On iron ore slope, Serra da Mutuca, beyond Barreiro, Mun. Nova Lima, Minas Gerais, Brazil, $1400 \mathrm{~m}$ alt, 15 April 1945, Williams \& Assis 6641 (GH, holotype).

Distribution.-Known only from the typecollection.

\section{Barbacenia culta L. B. Smith \& Ayensu, new species}

Plates 27, 37c,d

A B. mollis Goethart \& Henrard, cui verisimiliter affinis, floribus subduplo majoribus laxissime vestitis differt.

Caudex to $10 \mathrm{~cm}$ long, covered by old leaves. Leaf-sheaths covered; blades linear, filiformattenuate, $22 \mathrm{~cm}$ long, $16 \mathrm{~mm}$ wide, exceeding the flowers, sulcate on both sides with the furrows much broader than the nerves, laxly and minutely whitepubescent, ciliate with larger hairs with dark swollen but scarcely glandular apices.

Scapes $1-3$, to $8 \mathrm{~cm}$ long above the leaf-sheaths, laxly pubescent. Flowers laxly vestite with dark stipitate glands, dull red when dry. Ovary elliptic, distinct, ca. $12 \mathrm{~mm}$ long. Perianth-tube above ovary funnel-form and then cylindric, $50 \mathrm{~mm}$ long, 10 $\mathrm{mm}$ wide. Tepals nearly uniform, elliptic, $15 \mathrm{~mm}$ long. Anthers not seen; coronoid appendages semiorbicular with 2 short obtuse lobes, $2.5 \mathrm{~mm}$ long. Style scarcely enlarged at apex; stigmas apical, elongate.

Leaf Anatomy (Menezes 1).-Surface View: Hairs: fairly long multicellular hairs present on both surfaces. Epidermis: cells on both surfaces square to rectangular; thin-walled. Stomata: tetracytic, $24 \times 15 \mu \mathrm{m}$; present on both surfaces; more numerous on abaxial surface.

Transverse Section of Lamina: Dorsiventral; widely V-shaped with extreme margins turned slightly downward. Both surfaces undulating. Fairly long multicellular hairs present on both surfaces. Epidermis: cells on both surfaces small; rounded to dome-shaped; few conical; thin-walled. Cuticle: thin and smooth over entire surface. Stomata: present on both surfaces; stomata flush with epidermal surface; fairly large substomatal chamber present. Mesophyll: 3 layers of small, thin-walled palisade cells followed by 6 or 7 layers of rounded, loosely arranged spongy tissue. Cells fairly large and translucent above midvein. Vascular bundles: 59; commissural bundles observed. One large vessel present in most bundles. Two phloem units lying laterally in flanges of deep V- or Y- shaped abaxial girder. Deep inverted $Y$-shaped adaxial girder present on each bundle. Girders extend to epidermis. No adaxial cap on midvein. Each vascular bundle completely surrounded by a bundle sheath. Crystals: druses present in most mesophyll cells. Tannins: present.

TyPE.-Without locality, cultivated in the Jardim Botânico do Rio de Janeiro, Brazil, 13 November 
1965, Menezes 1 (US, holotype; SPF, isotype).

Distribution.-Unknown.

\section{Barbacenia leucopoda L. B. Smith}

Barbacenia leucopoda L. B. Smith, Contr. U.S. Nat. Herb., 35:282, pl. 10: figs. 29, 30, 1962.

Aylthonia leucopoda (L. B. Smith) Menezes, Ciência e Cultura, 23(3):[421], 1971.

Type.-In campo, Serra do Cipó, Mun. [Conceição do] Mato Dentro, Minas Gerais, Brazil, 15 January 1951, Macedo 2999 (UC, holotype).

Distribution.-Known only from the typecollection.

99. Barbacenia sordida L. B. Smith \& Ayensu, new species

Plates 28, 37e,f

A $B$. blackii L. B. Smith, cui parum affinis, scapis floribusque valde viscosis, perianthii tubo tepalisque angustioribus differt.

Caudex short, few-branched (!Irwin et al.), covered by old leaves. Leaves sulcate on both sides, viscous; sheaths covered; blades linear-lanceolate, filiform-attenuate, constricted toward base, $32 \mathrm{~cm}$ long, $20 \mathrm{~mm}$ wide, densely vestite with fine white hairs ca. $0.5 \mathrm{~mm}$ long and scattered hairs $4-5 \mathrm{~mm}$ long, the furrows as wide or wider than the nerves when dry.

Scapes 3, about equaling the leaves, vestite like the leaves but the hairs mostly gland-tipped and a larger proportion of the long ones. Flowers viscous, vestite like the scapes, pinkish cream (IIrwin et al.). Ovary ellipsoid, distinct, $20 \mathrm{~mm}$ long, much enlarged in fruit. Perianth-tube above ovary cylindric, $25 \mathrm{~mm}$ long, $3 \mathrm{~mm}$ wide. Tepals nearly uniform, narrowly lanceolate, $20 \mathrm{~mm}$ long. Anthers ca. 12 $\mathrm{mm}$ long; coronoid appendages suboblong, $9 \mathrm{~mm}$ long, bidentate. Style exceeded by the stamens, slightly conical-thickened at apex with 3 linear confluent stigmas.

LeAF ANATomy (Irwin et al. 23673).-Surface View: Hairs: multicellular and tufts of hairs present on both surfaces. Epidermis: cells on both surfaces square to rectangular; thin-walled. Stomata: tetracytic; $21 \times 15 \mu \mathrm{m}$; present on both surfaces.

Transverse Section of Lamina: Dorsiventral; very widely V-shaped. Adaxial surface undulating; abaxial surface with small furrows. Multicellular hairs and tufts of hairs present on both surfaces. Epidermis: cells on both surfaces rounded to domeshaped; few oval; thin-walled. Cuticle: thin and smooth over entire surface. Stomata: present on both surfaces; stomata flush with epidermal surface; substomatal chamber present. Mesophyll: 3 or 4 layers of compactly arranged, rounded cells followed by 1 or 2 layers of large, translucent cells. Below this occur 3 or 4 layers of compactly arranged, rounded cells. Cells larger and translucent above midvein. Vascular bundles: 29; commissural bundles observed. One or two large vessels present in each bundle, mostly one. Two phloem units lying laterally in flanges of short Y-shaped abaxial girder. Inverted $Y$-shaped adaxial girder present on each bundle. Bundle sheath completely surrounding each vascular bundle; few sheath cells quite large. Crystals: druses present in mesophyll. Tannins: present.

TYPE.-Sandstone precipices and adjacent cerrado, ca. $18 \mathrm{~km}$ west of Grão Mogul, Minas Gerais, Brazil, $950 \mathrm{~m}$ alt, 21 February 1969, Irwin, Reis dos Santos, Souza \& Fonseca 23673 (US, holotype; NY; $\mathrm{UB}$, isotypes).

Distribution.-Brazil: Minas Gerais: On brown sand in cerrado, type-locality, 18 February 1969, Irwin et al. 23533 (NY, UB, US). This latter has somewhat smaller leaves than the type but somewhat differently shaped coronoid appendages and a style that exceeds the stamens. It is possible that this species has heterostylous flowers.

\section{Barbacenia blackii L. B. Smith}

Barbacenia blackii L. B. Smith, Contr. U.S. Nat. Herb., 35:283, pl. 10: figs. 31, 32, 1962.-Ayensu, Smithsonian Contr. Bot., 15:61, pls. 9c,d, 48c, 1974.

Aylthonia blackii (L. B. Smith) Menezes, Ciência e Cultura, $23(3):[421], 1971$.

Type.-Serra do Cipó, Minas Gerais, Brazil, 13 January 1951, Pires \& Black 2734 (US, holotype).

Distrıbutıon.-Brazil: Minas Gerais: Conceição do Mato Dentro.

\section{Barbacenia rubra L. B. Smith}

Barbacenia rubra L. B. Smith, Contr. U.S. Nat. Herb., 35:283, pl. 10: figs. $33,34,1962$. 
Aylthonia rubra (L. B. Smith) Menezes, Ciência e Cultura, 23(3):[421], 1971.

TyPe.-On rocky slopes, Serra da Mutuca, Mun. Nova Lima, Minas Gerais, Brazil, February 1945, Williams $5432 a$ ( $\mathrm{GH}$, holotype).

Distribution.-Known only from the typecollection.

\section{Barbacenia damaziana Beauverd}

Barbacenia damaziana Beauverd, Bull. Herb. Boiss. II, 5:1078, 1905.-L. B. Smith, Contr. U.S. Nat. Herb., 35:284, 1962.

Aylthonia damaziana (Beauverd) Menezes, Ciência e Cultura, 23(3):[421], 1971.

Type.-On rocks, plateau of Itacolumí, 23 July 1905, Damazio 1778 (G, holotype (?) n v).

Distribution.-Known only from the typecollection.

\section{Barbacenia bahiana L. B. Smith}

Barbacenia bahiana L. B. Smith, Contr. U.S. Nat. Herb., $35: 284,1962$.
Aylthonia bahiana (L. B. Smith) Menezes, Ciência e Cultura, $23(3):[421], 1971$.

Type--Vicinity of Machado Portelo, Bahia, Brazil, 19-23 June 1915, Rose \& Russell 19924 (US, holotype).

Distribution.-Known only from the typecollection.

\section{Barbaceniopsis L. B. Smith}

Barbaceniopsis L. B. Smith, Contr. U.S. Nat. Herb., 35:270, 1962.-Ayensu, Am. Journ. Bot., 55:399, 1968.-Noher, Kurtziana, 5:293, fig. 1, 1969.

Plants short-caulescent, simple or branched, dioecious. Leaf-blades long-persistent.

Flowers functionally unisexual. Perianth forming a tube above the ovary. Tepals subequal. Stamens 6; filaments highly fused with the tepals; anthers dorsifixed, appearing sessile; coronoid appendages lacking. Stigmas vertical.

LeAF ANATOMY.-Blades about equally furrowed on the sides, the bundle sheaths close to the epidermis on both sides.

TYPE-SPECIES.-Vellozia boliviensis Baker.

\section{Key to the Species of Barbaceniopsis}

1. Leaf-blades even, cinereous beneath; all tepals merely acute.

2. Flowers lilac-violet, the male ca. $40 \mathrm{~mm}$ long, the female ca. $45 \mathrm{~mm}$ long; inner tepals glabrous; ovary trichomes imbricate, $1.5-2 \mathrm{~mm}$ long; fruit ca. $35 \mathrm{~mm}$ long. Southern

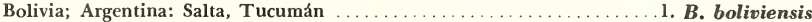

2. Flowers yellowish, the male ca. $35 \mathrm{~mm}$ long, the female ca. $40 \mathrm{~mm}$ long; inner tepals partially pubescent; ovary trichomes not imbricate, $0.6-0.9 \mathrm{~mm}$ long; fruit ca. $15 \mathrm{~mm}$

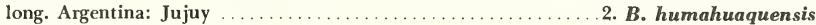

1. Leaf-blades carinate, green beneath, $3.5 \mathrm{~mm}$ wide; outer tepals long-aristate. Southern Peru

3. B. vargasiana

\section{Barbaceniopsis boliviensis (Baker) L. B. Smith}

Barbaceniopsis boliviensis (Baker) L. B. Smith, Contr. U.S. Nat. Herb., 35:271, 1962.-Ayensu, Am. Journ. Bot., 55:400, 1968.-Noher, Kurtziana, 5:293, fig. 1, 1969.

Vellozia boliviensis Baker, Mem. Torrey Bot. Club, 6:126, 1896.

Barbacenia boliviensis (Baker) Hauman, Anal. Mus. Hist. Nat. Buenos Aires, 29:429, 1917.

Barbacenia castilloni Hauman, Anal. Mus. Hist. Nat. Buenos Aires, 29:426, pl. 4, 1917 [type: El Bañado, Quebrada de las Cañas, valley of Calchaquí, Dept. Tafí, Prov. Tucumán, Argentina, $2140 \mathrm{~m}$ alt, February 1913, Castillon 3245 (LIL 13.154 type)].

Xerophyta boliviensis (Baker) Menezes, Ciência e Cultura,
23(3):[421], 1971; Bol. Zool. e Biol. Mar. ser. 2, 30:718, 719 figs. 115-118, 1973 .

TYPE.-Near Cochabamba, Dept. Cochabamba, Bolivia, 1891, Bang 1134 (K, holotype; US, isotype).

Distribution.-Southern Bolivia, Argentina: Salta, Tucumán.

\section{Barbaceniopsis humahuaquensis Noher}

Barbaceniopsis humahuaquensis Noher, Kurtziana, 7:262, 1973.

Type.-Between León and Yala, Dept. Capital, 
Prov. Jujuy, Argentina, 12 December 1972, Subils \& Noher 1666 (CORD, staminate plant).

Distribution.-Argentina: Jujuy.

\section{Barbaceniopsis vargasiana (L. B. Smith)} L. B. Smith

Barbaceniopsis vargasiana (L. B. Smith) L. B. Smith, Contr. U.S. Nat. Herb., 35:271, 1962.-Ayensu, Am. Journ. Bot., 55:401, 1968.

Barbacenia vargasiana L. B. Smith, Bol. Soc. Peru Bot., 1:13, figs. 1, 2, 1948.-C. Vargas C., Biota 3, 28:353, 1961.

Xerophyta vargasiana (L. B. Smith) Menezes, Ciência e Cultura, 23(3):422, 1971.

Type.-Anta, between Sisal and Cunyac, Prov. Anta, Dept. Cuzco, Peru, $2100 \mathrm{~m}$ alt, 18 January 1945, C. Vargas C. 4883 (GH, holotype; US, isotype).

Distribution.-Peru: Apurimac, Cuzco.

\section{Subfamily VELLOZIOIDEAE Menezes}

Vellozioideae Menezes, Ciência e Cultura, 23(3):[421], 1971 [emend. L. B. Smith \& Ayensu].

Stigmata horizontalia vel paulo reflexa suborbicularia. Stamina raro 6 plerumque plura.

Floral appendages when present always ventral to the stamens. Stamens 6 or usually more; filaments always evident and cylindrical, anthers typically basifixed; pollen in tetrads. Stigmas horizontal to somewhat reflexed, always apical and apically confluent, suborbicular.

Adaxial epidermis not in contact with the bundle sheath.

Note: Original description emended to include species with 6 stamens and horizontal stigmas that otherwise accord with the characters cited by Menezes.

\section{Nanuza L. B. Smith \& Ayensu, new genus}

Vellozia sensu Martius, Nov. Gen. \& Sp., 1:16, pl. 9, 1823 [in part, not as to type].

Xerophyta sensu Sprengel, Syst. Veg. 4: Cur. Post. 137, 1827 [in part, not as to type].

Caulescens; caudice foliorum vaginis vetustis omnino obtecto itaque trigono; foliis novellis termi- nalibus, laminis latis, plicatis, demum regulariter deciduis.

Flores perfectos; perianthii tubo epigyno nullo, tepalis subaequalibus; staminibus 6, filamentis teretibus; antheris basifixis auriculatisque, pollinis granis discretis; stigmatibus subhorizontalibus orbicularibus apice cohaerentibus.

Folii lamina secta: latere adaxiali quam abaxiali profundiore excisa, epidermide adaxiali fasciculi vaginam haud contigua.

Type-Species.-Vellozia plicata Martius.

The authors take pleasure in dedicating this new genus to Doctora Nanuza Luiza de Menezes, whose research is both a challenge and a great help to her colleagues.

\section{Nanuza plicata (Martius) L. B. Smith \&c Ayensu, new combination}

Figure 1

Vellozia plicata Martius, Nov. Gen. \& Sp., 1:16, pl. 9, 1823.Seubert in Martius, Fl. Bras., 3(1):74, 1847.-L. B. Smith, Contr. U.S. Nat. Herb., 35:259, 1962.-Ayensu, Smithsonian Contr. Bot., 15:35, fig. 4f, pl. 44a, 1974.

Xerophyta plicata (Martius) Sprengel, Syst. Veg. 4: Cur. Post. 137, 1827.-Baker, Journ. Bot., 13:236, 1875.-Menezes, Bol. Zool. \& Biol. Mar. ser. 2, 30:718, figs. 82-113, 1973.

Vellozia triquetra Pohl, P1. Bras., 1:129, 1827.-Seubert in Martius, Fl. Bras., 3(1):74, 1847.-L. B. Smith, Contr. U.S. Nat. Herb., 35:285, 1962.-Ayensu, Smithsonian Contr. Bot., 15:42, pl. 44f, 1974 [type: granitic soil, between Inhumaes and Quartel do Teixeira, Minas Gerais, Brazil, [September 1820], Pohl s n (W, lost, sterile)].

Xerophyta triquetra (Pohl) Baker, Journ. Bot., 13:236, 1875.

TYPE.-Fields derived from micaceous schist, Serra de Monte Santo (ca. $10^{\circ} 26^{\prime} \mathrm{S}, 39^{\circ} 20^{\prime} \mathrm{W}$ ), Bahia, Brazil, $450 \mathrm{~m}$ (1500 ft), April-May [1819], Martius s $n$ (M, holotype (?) $\mathrm{n}$ v).

Distribution.-Brazil: Bahia: Rocky slope near Milagres, s d, Menezes 210 (SPF, US). On limestone outcrop $6 \mathrm{~km}$ south of Cocos, $520 \mathrm{~m}$ alt, 16 March 1972, Anderson, Stieber \& Kirkbride 37020 (NY, UB, US). Espírito Santo: (and Minas Gerais) Nanuque and Serra dos Aymores, 10 November 1953, Duarte 3635 \& Gomes 420 (RB, US). Morro de Agá, Piuma, 25 July 1965, Menezes, Oliveira Filho \& Oliveira 4 (SPF, US); idem, 8 August 1969, Menezes \& Sazima 54 \& 55 (SPF, US): idem, 8 September 1971, Cabral \& Oliveira Filho s $n$ (SPF, US). Minas Gerais: Teofilo Otoni, Magalhães 14099 (NY). 


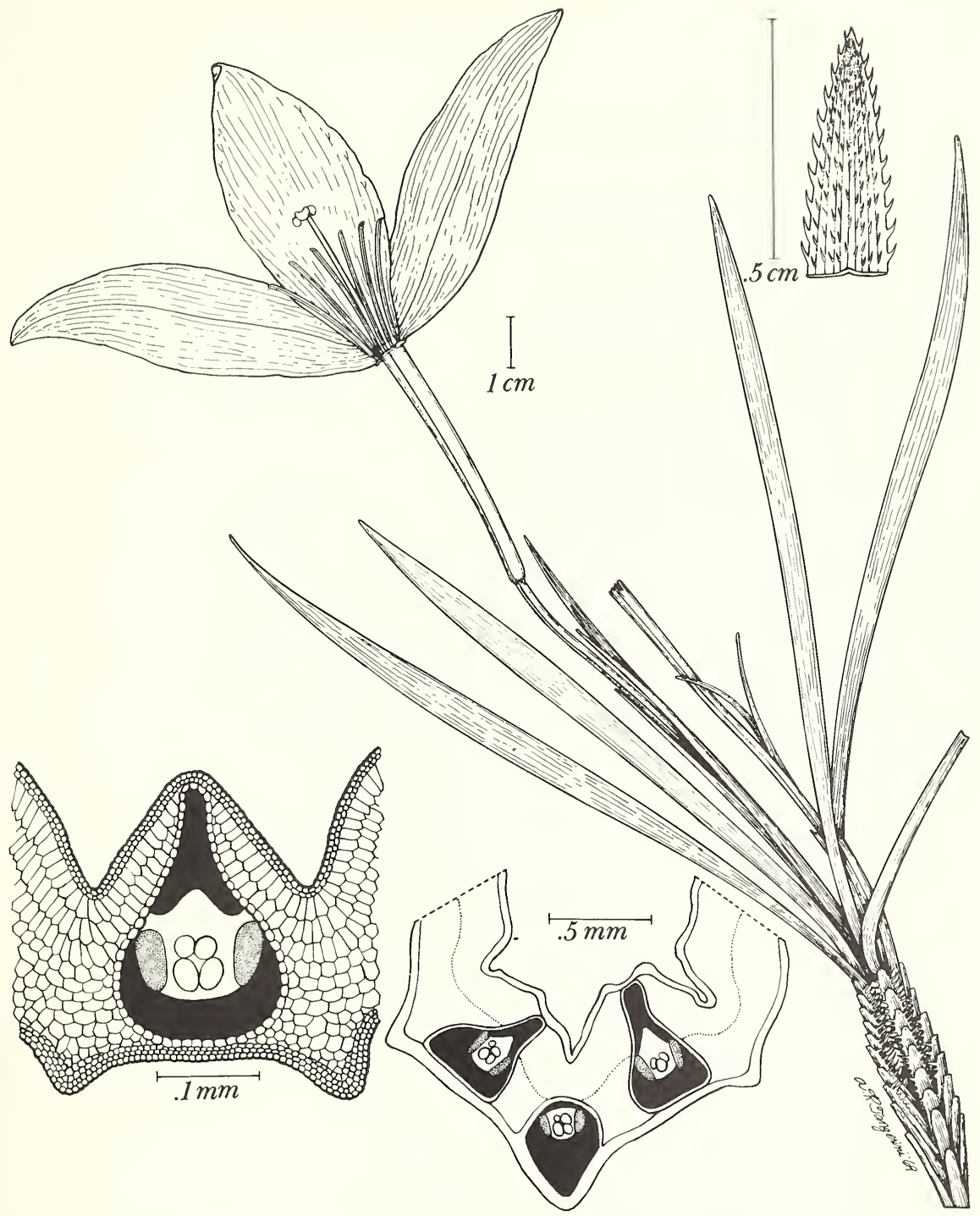

Figure 1.-Nanuza plicata (Martius) L. B. Smith \& Ayensu, new combination (Menezes \& Sazima 55). 


\section{Vellozia Vandelli}

Vellozia Vandelli, F1. Lusit. \& Brasil. Spec., 32, pl. 2, 1788.Seubert in Martius, Fl. Bras., 3(1):73, 1847.-L. B. Smith, Contr. U.S. Nat. Herb., 35:253, 1962.-Ayensu, Smithsonian Contr. Bot., 15:10, 1974.-L. B. Smith, Taxon, 24(4):474, 1975.

Campderia A. Richard, Bull. Soc. Philom., 79, 1822 [based on C. langsdorfi A. Richard and C. tubiflora A. Richard].

Radia A. Richard, in Kunth, Syn. Pl. Aequin., 1:300, 1822 [type: $R$. tubiflora A. Richard].

Xerophyta sensu Martius ex Schultes f. in Roemer \& Schultes, Syst., 7:290, 1826.- Sprengel, Syst. Veg., 4: Cur. Post. 137, 1827.-Baker, Journ. Bot., 13: 236, 1875.-Menezes, Ciência e Cultura, 23(3):422, 1971 [in part, as to New World species, not as to type].

Plant from small stature to over $4 \mathrm{~m}$ high. Caudex mostly few to many-branched, terete including the old leaf-bases. Leaf-blades reflexing and persistent or deciduous along a regular transverse line, not plicate, even or nearly so on the adaxial side.

Perianth-tube from equaling the ovary to several times as long. Stamens 6 or more numerous and in 6 flat bundles or phalanges; filaments terete and filamentous; anthers basifixed; pollen in tetrads. Ovary from depressed at apex to forming a distinct epigynous tube distinguishable from a perianthtube by its firmer texture. Style elongate; stigmas horizontal or somewhat reflexed, suborbicular, confluent at apex.

Adaxial epidermis not in contact with the bundle sheath.

TYPE-SPECIES.-Vellozia glabra Mikan.

\section{Key to the Sections and Subkeys of Vellozia}

1. Tepals free or if adherent then for 5-30 $\mathrm{mm}$ with their margins distinct and separating with age; filaments free.

2. Leaf-blades subterete (conduplicate with each side semiterete), 1-2 mm wide, 12-25 mm long, strict, mostly regularly deciduous; furrows absent or very small, vascular bundles 6-12; caudex slender and mostly branched (ericoid); stamens 6 (Vellozia section Xerophyta Seubert in part; Xerophyta auctt. in part) ........ Section Xerophytoides, new section

2. Leaf-blades distinctly flattened, more than $2 \mathrm{~mm}$ wide or more than $25 \mathrm{~mm}$ long or usually both; furrows usually strong on abaxial side, vascular bundles mostly more than 12; caudex mostly stout; stamens mostly more than $6 \ldots \ldots \ldots \ldots 2$. Section Vellozia

3. Ovary or capsule glabrous or laxly vestite and visible between the trichomes.

4. Trichomes acute and nonglandular or the ovary and scape glabrous .... Subkey I

4. Trichomes glandular $\ldots \ldots \ldots \ldots \ldots \ldots \ldots \ldots \ldots \ldots \ldots \ldots \ldots \ldots \ldots \ldots \ldots \ldots$ Subkey II

3. Ovary or capsule completely covered by its trichomes.

5. Trichomes acute or truncate, nonglandular $\ldots \ldots \ldots \ldots \ldots \ldots \ldots$ Subkey III

5. Trichomes glandular $\ldots \ldots \ldots \ldots \ldots \ldots \ldots \ldots \ldots \ldots \ldots \ldots \ldots \ldots \ldots \ldots \ldots \ldots \ldots \ldots \ldots \ldots$ Subkey IV

1. Tepals forming a long slender tube $35-80 \mathrm{~mm}$ long above the ovary with their margins indistinguishable, circumscissile in age (except $V$. caput-ardeae); filaments adnate or agglutinated to the tube but partly distinguishable .......... Section Radia, new status

\section{Key to the Species of Vellozia}

\section{Section Xerophytoides}

(Brazil: Minas Gerais)

1. Leaf-blades ciliate; sheaths pubescent; ovary obconic, glabrous; spongy tissue making up nearly all of mesophyll $\ldots \ldots \ldots \ldots \ldots \ldots \ldots \ldots \ldots \ldots \ldots \ldots \ldots \ldots \ldots \ldots \ldots \ldots \ldots \ldots \ldots \ldots \ldots$

1. Leaf-blades serrulate or entire; some palisade tissue evident on adaxial side of mesophyll.

2. Ovary glabrous, obconic; palisade tissue grading into spongy tissue ..2. V. tragacantha

2. Ovary vestite.

3. Leaf-blades entire, glutinous; palisade tissue distinct $\ldots \ldots \ldots \ldots \ldots \ldots$. V. scoparia

3. Leaf-blades serrulate; palisade tissue indistinct.

4. Ovary globose, its glands generally and laxly distributed; leaf-blades soon decidu-

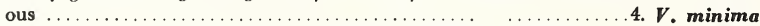

4. Ovary obovoid, its glands in lines; leaf-blades tardily deciduous . . . 5. V. virgata 


\section{Section Vellozia}

\section{SUBKeY I}

1. Scapes covered by the leaf-sheaths. Brazil: Minas Gerais.

2. Ovary without ribs or with flat intervals between them; leaf-blades subacute to retuse.

3. Leaf-blades densely long-ciliate at base, to $10 \mathrm{~mm}$ wide; furrows papillate . 6. V. ciliata

3. Leaf-blades serrulate throughout.

4. Leaf-blades to $3.5 \mathrm{~cm}$ long, strongly retuse; furrows smooth . . . . 7. V. taxifolia

4. Leaf-blades $9-20 \mathrm{~cm}$ long, subobtuse or subacute or obscurely retuse

8. V. cryptantha

2. Ovary covered with numerous rounded ribs; leaf-blades $4 \mathrm{~cm}$ long, $3.5 \mathrm{~mm}$ wide, abruptly acute and cuspidate

1. Scapes exserted above the leaf-sheaths.

5. Ovary covered with numerous rounded ribs; leaf-blades retuse, to $6 \mathrm{~cm}$ long; sclerenchyma beneath abaxial epidermis almost continuous. Brazil: Minas Gerais

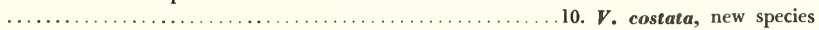

5. Ovary without ribs or with flat intervals between them.

6. Ovary completely glabrous or rarely very sparsely and obscurely acute-verrucose $(V$. tenella); scapes glabrous.

7. Ovary alate-trigonous; capsule $5 \mathrm{~cm}$ long; tepals clawed, $60-105 \mathrm{~mm}$ long; leaf cells above furrows larger and thicker walled than the rest of the mesophyll. Brazil:

Minas Gerais

11. V. alata

7. Ovary not at all alate.

8. Ovary broad, subglobose or broadly obconic; leaf-sheaths narrow; caudex slender, simple, short. Brazil: Minas Gerais.

9. Stamens 6 , unappendaged.

10. Leaf-blades attenuate to acute; caudex distinct; abaxial furrows only slight undulations $\ldots \ldots \ldots \ldots \ldots \ldots \ldots \ldots \ldots \ldots \ldots \ldots \ldots$. V. sellovii

10. Leaf-blades rounded-retuse; caudex very short . . . . . . 13. V. laevis

9. Stamens more than 6 .

11. Stamens subequal, unappendaged ............. V. macedonis

11. Stamens very unequal, appendaged at base; abaxial furrows about $1 / 2$ thickness of leaf-blade .................... tenella

8. Ovary distinctly longer than wide, mostly trigonous.

12. Apices of the old leaf-sheaths much exposed, persistently smooth, lustrous and brown; blades attenuate; hairs on abaxial furrows; tepals to $70 \mathrm{~mm}$ long; stamens ca. 18. Brazil: Minas Gerais ............. V. glabra

12. Apices of the old leaf-sheaths very little exposed, soon rough and dull.

13. Leaf-blades subacute to retuse. Brazil: Minas Gerais.

14. Flowers much exceeded by the leaves.

15. Flowers erect, largely covered by the leaves ....17. V. pusilla

15. Flowers decurved; leaf: sclerenchyma cells subjacent to adaxial epidermis

18. $V$. incurvata

14. Flowers erect, not exceeded by the leaves; leaf sclerenchyma cells separated from adaxial epidermis; stamens ca. 30 or more. Brazil: Minas Gerais ....................41. V. compacta 13. Leaf-blades attenuate.

16. Ovary strongly trigonous; tepals elliptic; stamens ca. 30 or more; leaf furrows with small hairs. Bolivia, Brazil: Minas Gerais, Goiás,

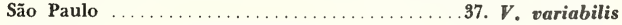

16. Ovary terete; tepals oblanceolate; stamens 40-50; leaf furrows glabrous.

17. Caudex 2-5 cm long; leaf-blades $15 \mathrm{~cm}$ long; abaxial girder sclerenchyma tapered at base; tepals $15 \mathrm{~mm}$ long. Brazil: Minas Gerais ................ 19. V. brachypoda, new species

17. Caudex 1-4 m long; leaf-blades $45 \mathrm{~cm}$ long; abaxial girder sclerenchyma spreading laterally at base; tepals $70 \mathrm{~mm}$ long. Brazil: Bahia ............20. V. sincorana, new species

6. Ovary partially vestite with acute nonglandular trichomes. 
18. Trichomes confined to the base and angles of the ovary.

19. Leaf-blades attenuate. Brazil: Minas Gerais, Goiás, Mato Grosso

21. V. verruculosa

19. Leaf-blades subacute to retuse. Brazil: Minas Gerais.

20. Old leaf-sheaths mostly entire; leaves exceeding the flowers

$\ldots \ldots \ldots \ldots \ldots \ldots \ldots \ldots \ldots \ldots \ldots \ldots \ldots \ldots \ldots \ldots \ldots \ldots \ldots \ldots$

20. Old leaf-sheaths splitting into coarse fibers.

21. Tepals $30 \mathrm{~mm}$ long; leaves exceeding the flowers ....22. V. fibrosa

21. Tepals $50 \mathrm{~mm}$ long; flowers exceeding the leaves ....23. $V$. crispata

18. Trichomes evenly distributed over the ovary.

22. Tepals 40-60 mm long; caudex mostly simple.

23. Tepals spatulate or oblanceolate with a claw, 50-60 mm long; caudex elongate; leaf furrows papillate.

24. Ovary triquetrous; tube above it very short; adaxial leaf surface slightly ridged. Brazil: Bahia ...........

24. Ovary terete; tube above almost as long as ovary; adaxial leaf surface very slightly undulating. Brazil: Minas Gerais .............. $\ldots \ldots \ldots \ldots \ldots \ldots \ldots \ldots \ldots \ldots \ldots \ldots \ldots \ldots \ldots$. V. hatschbachii, new species

23. Tepals elliptic or lanceolate without claw, 40-50 mm long; caudex simple, short. Brazil: Minas Gerais.

25. Ovary ovoid or ovoid-globose; leaf furrows smooth ..26. V asperula

25. Ovary oblong-ellipsoid $\ldots \ldots \ldots \ldots \ldots \ldots \ldots \ldots \ldots \ldots$ 27. V. bradei

22. Tepals 10-30 mm long; caudex mostly branched. Brazil: Minas Gerais.

26. Leaf-sheaths splitting into coiled fibers; margins of the blades serrulate; 1-3 layers of parenchyma cells subjacent to adaxial epidermis.

27. Tepals $20 \mathrm{~mm}$ long; leaf-blades $3-4 \mathrm{~mm}$ wide; palisade cells changing abruptly to spongy tissue $\ldots \ldots \ldots \ldots \ldots \ldots \ldots \ldots \ldots 28 . \boldsymbol{V}$. ornata

27. Tepals $10 \mathrm{~mm}$ long; leaf-blades $4-7 \mathrm{~mm}$ wide; palisade cells grading into spongy tissue $\ldots \ldots \ldots \ldots \ldots \ldots \ldots \ldots \ldots \ldots 29 . \quad$ V. granulata

26. Leaf-sheaths remaining entire.

28. Blades entire, not obviously bicarinate above, to $18 \mathrm{~cm}$ long and 12 mm wide; adaxial surface undulating in section ....30. $\boldsymbol{V}$. piresiana

28. Blades finely serrulate, often bicarinate above.

29. Branch apices including old leaf-sheaths 3-6 $\mathrm{mm}$ thick; blades 2-5 cm long; adaxial furrows even; scapes much shorter than the leaves and decurved after anthesis ........60. V. declinans

29. Branch apices including old leaf-sheaths $15-25 \mathrm{~mm}$ thick; blades to $9 \mathrm{~cm}$ long.

30. Sheaths exposed and lustrous-castaneous at apex; blades tardily deciduous; adaxial furrows papillate ............

31. V. castanea, new species

30. Sheaths almost wholly covered, soon dull and weathered; blades quickly deciduous.

31. Ovary stoutly ellipsoid

32. V. ramosissima

31. Ovary triquetrous with flat sides

33. V. bicarinata, new species

\section{SUBKeY II}

1. Ovary unevenly vestite with trichomes at base or apex or in vertical lines.

2. Leaf-blades long-attenuate or caudate-acuminate, narrowly triangular.

3. Ovary broadly winged; leaf: furrows papillate; abaxial sclerenchyma layer next to epidermis. Brazil: Minas Gerais ..............

3. Ovary not more than angled.

4. Blades $3 \mathrm{~mm}$ wide but to $18 \mathrm{~cm}$ long; leaf: furrows smooth, no abaxial sclerenchyma layer; caudex short, mostly simple; ovary glands sessile or subsessile. Brazil: Minas

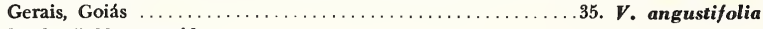
4. Blades 5-18 $\mathrm{mm}$ wide. 
5. Ovary triquetrous; leaf: large translucent cells in adaxial layer of mesophyll with radial extensions to furrows and bundles.

6. Glands of ovary slenderly stipitate. Brazil: Minas Gerais .36. V. caruncularis

6. Glands of ovary sessile or subsessile. Bolivia, Brazil: Minas Gerais, Goiás,

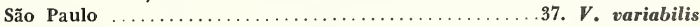

5. Ovary terete or subterete or 3-lobed with subterete carpels. Brazil: Minas Gerais.

7. Glands of the ovary slenderly stipitate; abaxial sclerenchyma layer lacking

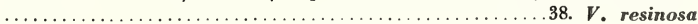

7. Glands of the ovary sessile or subsessile; abaxial sclerenchyma layer next to epidermis.

8. Blades $6 \mathrm{~mm}$ wide; scape continuous ........ 39 . teres, new species

8. Blades $12 \mathrm{~mm}$ wide; scape articulate near apex

40. V. torquata, new species

2. Leaf-blades subacute to retuse, sublinear. Brazil: Minas Gerais.

9. Blades erect, soon deciduous; furrows smooth; ovary subterete.

10. Caudex tall, stout, branched; blades $7 \mathrm{~mm}$ or wider; tepals suberect at anthesis 41. $V$. compacta

10. Caudex very short, simple; blades $2.5 \mathrm{~mm}$ wide; tepals reflexed at anthesis ......

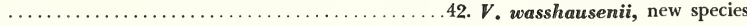

9. Blades soon reflexed, persistent, $3-8 \mathrm{~mm}$ wide.

11. Ovary glands slenderly stipitate; ovary strongly trigonous.

12. Blades glutinous; caudex long, slender, branching

43. V. fruticosa

12. Blades dry; caudex short, simple or fasciculate-branching; leaf: furrows papil-

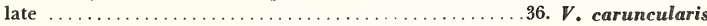

11. Ovary glands sessile or subsessile; leaf: furrows papillate.

13. Ovary tricostate; scapes solitary; leaf-blades $5.5 \mathrm{~cm}$ long, $4.5 \mathrm{~mm}$ wide..... $\ldots \ldots \ldots \ldots \ldots \ldots \ldots \ldots \ldots \ldots \ldots \ldots \ldots \ldots \ldots \ldots \ldots \ldots \ldots \ldots \ldots \ldots$. patens, new species

13. Ovary terete; scapes several; leaf-blades $12(-18) \mathrm{cm}$ long, $12 \mathrm{~mm}$ wide $\ldots . .$. $\ldots \ldots \ldots \ldots \ldots \ldots \ldots \ldots \ldots \ldots \ldots \ldots \ldots \ldots \ldots \ldots \ldots \ldots$. modesta, new species

1. Ovary evenly and laxly vestite.

14. Leaf-blades long-attenuate, very narrowly triangular or acute and caudate. Brazil: Minas Gerais.

15. Ovary trichomes $3 \mathrm{~mm}$ long, slender; sheaths dark brown, lustrous; blades reflexed, persistent; large translucent cells in adaxial layer of mesophyll with radial extensions to furrows and bundles; furrows papillate; scape short, decurved . 46. V. breviscapa

15. Ovary trichomes much less than $3 \mathrm{~mm}$ long.

16. Blades pilose- or setose-ciliate.

17. Ovary oblong ....................

17. Ovary globose to obovoid.

18. Ovary broad at apex, broadly truncate; leaf: large translucent cells radial above vascular bundles and furrows ....48. $V$. barbaceniifolia

18. Ovary contracted at apex.

19. Capsule $15 \mathrm{~mm}$ long, the carpels terete ....... 49. Viannae

19. Capsule $30 \mathrm{~mm}$ long, the carpels subalate; large translucent cells radial above vascular bundles and furrows

50. V. subalata, new species

16. Blades not more than serrulate.

20. Blades $10-15 \mathrm{~mm}$ wide, reflexed, persistent; large translucent cells in adaxial layer of mesophyll with radial extensions to furrows and bundles, furrows papillate.

21. Ovary oblong, strongly angled; leaf: sclerenchyma layer next to abaxial epidermis . ........................ V. intermedia

21. Ovary ellipsoid, not angled; leaf: no sclerenchyma layer next to abaxial spidermis .................. 5 . tomeana, new species

20. Blades only about $3 \mathrm{~mm}$ wide.

22. Sheaths densely imbricate; blades reflexed, persistent $\ldots \ldots \ldots \ldots \ldots \ldots \ldots \ldots \ldots \ldots \ldots \ldots \ldots . \ldots \ldots$. graomogulensis

22. Sheaths laxly imbricate; blades divergent, then deciduous; large translucent cells radial above vascular bundles only; furrows papillate .... 
14. Leaf-blades subacute to retuse.

23. Sheaths densely imbricate, their apices very little exposed. Brazil: Minas Gerais.

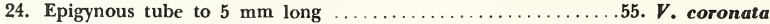

24. Epigynous tube very short or none $\ldots \ldots \ldots \ldots \ldots \ldots \ldots \ldots \ldots$ V. brevifolia

23. Sheaths laxly imbricate, their apices much exposed.

25. Leaf-blades pilose throughout, $1.5 \mathrm{~mm}$ wide; adaxial surface distinctly ridged, furrows small, large translucent cells above vascular bundles. Brazil: Minas

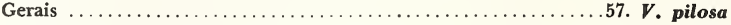

25. Leaf-blades incompletely if at all vestite.

26. Sheaths ciliate throughout with stiff spreading setae. Brazil: Minas Gerais

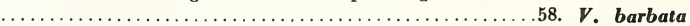

26. Sheaths entire or with a few teeth toward apex.

27. Blades contorted, subdensely setose. Brazil: Minas Gerais

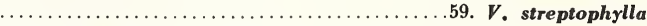

27. Blades straight.

28. Scape very short, decurved; large translucent palisade cells above vascular bundles and furrows. Brazil: Minas Gerais $\ldots \ldots \ldots \ldots$

28. Scape elongate, erect or nearly so.

60. V. declinans

29. Blades reflexed, persistent; caudex slender. Brazil: Minas Gerais $\ldots \ldots \ldots \ldots \ldots \ldots \ldots \ldots \ldots \ldots \ldots \ldots \ldots \ldots \ldots \ldots \ldots \ldots .6 \ldots \ldots$. marcescens

29. Blades erect, soon deciduous.

30. Caudex stout; blades to $30 \mathrm{~cm}$ long. Brazil: Minas Gerais $\ldots \ldots \ldots \ldots \ldots \ldots \ldots \ldots \ldots \ldots \ldots \ldots \ldots \ldots \ldots \ldots \ldots \ldots$. . spiralis

30. Caudex slender; blades much smaller; large translucent palisade cells above vascular bundles and furrows; furrows papillate.

31. Ovary strongly constricted at apex to the distinct epigynous tube; tepals broad, suberect at anthesis. Brazil: Minas Gerais ........63. V. epidendroides

31. Ovary scarcely constricted at apex, the epigynous tube almost none.

32. Glands of the ovary slenderly stipitate; tepals narrow, reflexed at anthesis. Brazil: Minas Gerais 64. V. leptopetala

32. Glands of the ovary subsessile; tepals broad, suberect at anthesis. Brazil: Espírito Santo, Rio de Janeiro (state), Minas Gerais ...65. V. variegata

\section{SUBKEY III}

1. Leaf-blades finely attenuate.

2. Scape glabrous or at most slightly scabrous at summit, contrasting sharply with the densely vestite ovary; leaf: large translucent cells radial above the bundles only; much adaxial and abaxial sclerenchyma.

3. Trichomes of the ovary stout, apiculate or truncate at anthesis and after; tepals ca. 8-14 cm long; leaf-sheaths remaining entire.

4. Trichomes prismatic, broadly truncate. Brazil: Bahia, Goiás, Mato Grosso

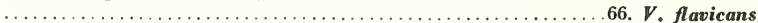

4. Trichomes ovoid, apiculate or minutely truncate. Brazil: Goiás, Mato Grosso ....

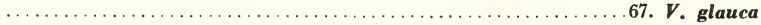

3. Trichomes of the ovary slender, subulate or laterally flattened at least at anthesis.

5. Leaf-sheaths remaining entire.

6. Sheaths barely exposed; caudex $12-20 \mathrm{~mm}$ thick. Brazil: Minas Gerais, Goiás

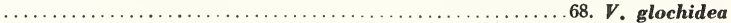

6. Sheaths much exposed; caudex 8-12 mm thick. Brazil: Minas Gerais

69. V. nuda, new species

5. Leaf-sheaths rapidly splitting into fibers. Brazil: Minas Gerais. 
7. Blades glabrous beneath except the keel, bicarinate above; ovary trichomes subulate; tepals white to blue ............... $70 . \quad$. crassicaulis

7. Blades villous beneath; ovary trichomes much flattened, obtuse; tepals yellow

71. V. sulphurea

2. Scape strongly vestite on the upper third or half, not contrasting with the ovary.

8. Leaf-blades 12-15 mm wide; tepals 50-120 mm long; scape muricate toward apex.

9. Blades densely and finely white-tomentose beneath, setose-ciliate; translucent cells

above furrows, no adaxial or abaxial sclerenchyma. Brazil: Minas Gerais

72. V. nivea, new species

9. Blades glabrous, serrulate.

10. Scape $7 \mathrm{~cm}$ long; ovary trichomes abruptly attenuate; leaf: large translucent cells above furrows, bundles and midvein; much adaxial and abaxial sclerenchyma. Brazil: Goiás, Mato Grosso ...............73. V. seubertiana

10. Scape to $40 \mathrm{~cm}$ long; ovary trichomes sharply truncate. Brazil: Bahia

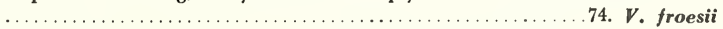

8. Leaf-blades $4-11 \mathrm{~mm}$ wide; scape $2-5 \mathrm{~cm}$ long above the leaf-sheaths.

11. Tepals $20 \mathrm{~mm}$ long; blades $4 \mathrm{~mm}$ wide, setose-ciliate; translucent cells radial above furrows and bundles, furrows papillate. Brazil: Goiás ....75. V. hypoxoides

11. Tepals $35-80 \mathrm{~mm}$ long; blades much more than $4 \mathrm{~mm}$ wide.

12. Sheaths remaining entire; tepals ca. 40-60 mm long.

13. Ovary trichomes truncate. Brazil: Bahia ........ 76. V. blanchetiana

13. Ovary trichomes fine-subulate. Brazil: Pará ...........77. V. bulbosa

12. Sheaths rapidly splitting into fibers.

14. Ovary trichomes fine, linear, flattened, spreading; leaf: translucent cells radial above both bundles and furrows. Brazil: Minas Gerais . . . . . . .

78. V. crinita

14. Ovary trichomes subulate, erect and closely appressed; leaf: translucent cells radial above bundles only.

15. Tepals $80 \mathrm{~mm}$ long. Brazil: Maranhão, Goiás .....79. V. swallenii

15. Tepals $35-40 \mathrm{~mm}$ long. Brazil: Goiás ........... 80. Vumila

1. Leaf-blades broadly subacute, obtuse or emarginate.

16. Scape glabrous, contrasting sharply with the densely vestite ovary; tepals to $90 \mathrm{~mm}$ long; sheaths much exposed, remaining entire.

17. Tepals $90 \mathrm{~mm}$ long, purple; blades glabrous; ovary trichomes simple, subulate, erect. Brazil: Minas Gerais ......................... pulchra

17. Tepals $50-70 \mathrm{~mm}$ long; blades vestite at least when young; translucent cells radial above bundles only.

18. Ovary trichomes simple, subulate or short-bidentate; blade trichomes simple, filamentous. Brazil: Bahia .............. 82. V. bahiana, new species

18. Ovary trichomes with forked recurving apices; blade trichomes plumose-lacerate. Brazil: Pernambuco ........................ 8 . cinerascens

16. Scape strongly vestite toward apex.

19. Trichomes of scape and ovary filamentous; leaf: translucent cells radial above bundles only. Brazil: Bahia $\ldots \ldots \ldots \ldots \ldots \ldots \ldots \ldots \ldots \ldots \ldots \ldots \ldots \ldots \ldots$. V. dasypus

19. Trichomes of scape and ovary much coarser.

20. Scape to $8 \mathrm{~cm}$ long above the leaf-sheaths; caudex ca. $15 \mathrm{~mm}$ thick; leaf: translucent cells above both bundles and furrows. Brazil: Minas Gerais

85. V. scabrosa, new species

20. Scape almost wholly covered by the leaf-sheaths. Brazil: Goiás.

21. Caudex ca. $2 \mathrm{~mm}$ thick; ovary trichomes simple, acute; scape terete; leaf: abaxial median section much extended on both sides ......86. V. exilis

21. Caudex to $15 \mathrm{~mm}$ thick; ovary trichomes in part bicuspidate; scape trigonous; leaf: abaxial median section not notably extended laterally

87. V. grisea

\section{SUBKEY IV}

1. Leaf-blades glabrous or nearly so, more or less serrulate.

2. Ovary distinctly broader than high, hemispheric; leaf: minor vascular bundles present above furrows. Brazil: Bahia. 
3. Caudex ca. $10 \mathrm{~mm}$ thick; leaf-sheaths covered

88. $V$. hemisphaerica

3. Caudex 3-4 mm thick, elongate, branched; sheaths exposed toward apex

89. V. burle-marxii, new species

2. Ovary at least as high as wide. Brazil: Minas Gerais.

4. Leaf-blades obtuse to retuse, bicarinate above

5. Sheaths much exposed; stems slender; blades erect, soon deciduous; translucent cells radial above both bundles and furrows.

6. Tepals reflexed at anthesis; filaments very dark; leaf: sclerenchyma layer next to baxial epidermis $\ldots \ldots \ldots \ldots \ldots \ldots \ldots \ldots \ldots \ldots \ldots \ldots \ldots . \ldots \ldots$. leptopetala

6. Tepals divergent at anthesis; filaments pale; leaf: no sclerenchyma layer next to epidermis ......................... $90 . \ldots$. lilacina, new species

5. Sheaths very little exposed; stems stout; blades reflexed, persistent.

7. Blades 8-12 $\mathrm{mm}$ wide, their margins densely pectinate-serrulate in several rows; translucent cells radial above both bundles and furrows $\ldots \ldots \ldots \ldots \ldots \ldots$

45. $V$. modesta, new species

7. Blades $3.5 \mathrm{~mm}$ wide, margins laxly serrulate in a single row ...91. V. arenicola

4. Leaf-blades finely attenuate.

8. Blades flat, over $10 \mathrm{~mm}$ wide; translucent cells above both bundles and furrows.

9. Ovary glands stipitate, dark; blades reflexed, persistent; sheaths almost wholly covered; furrows papillate $\ldots \ldots \ldots \ldots \ldots \ldots \ldots \ldots .92 . \boldsymbol{V}$. stipitata, new species

9. Ovary glands subsessile, pale; blades erect, deciduous; sheaths much exposed

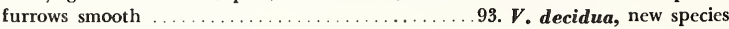

8. Blades revolute, $1.5-8 \mathrm{~mm}$ wide.

10. Tepals without claws.

11. Blades serrulate throughout, $6 \mathrm{~mm}$ wide; translucent cells above both bundles and furrows, furrows papillate

94. $V$. echinata

11. Blades serrulate only near apex, $1.5 \mathrm{~mm}$ wide; translucent cells above bundles only, furrows smooth

95. $V$. nanuzae, new species

10. Tepals with claws ca. $10 \mathrm{~mm}$ long adherent in a tube; leaf: no translucent cells above furrows.

12. Caudex very short, mat-forming; sheaths almost wholly covered; abaxial sclerenchyma at corners of furrows; tepals violet, $50 \mathrm{~mm}$ long. Brazil: Minas Gerais

96. $V$. geotegens, new species

12. Caudex to $1.5 \mathrm{~m}$ high, solitary; sheaths apically much exposed; no abaxial sclerenchyma; tepals white, $70-80 \mathbf{m m}$ long. Brazil: Rio de Janeiro (city and state) $\ldots \ldots \ldots \ldots \ldots \ldots \ldots \ldots \ldots \ldots \ldots \ldots \ldots \ldots \ldots . \ldots \ldots$. candida

1. Leaf-blades vestite, at least beneath, entire or toward apex glandular, but never serrulate; translucent cells above both bundles and furrows. Brazil: Minas Gerais.

13. Blades vestite beneath with straight, divergent to spreading hairs, the nerves visible.

14. Caudex to $2 \mathrm{~m}$ high, branched; blades vestite beneath with erect hairs, narrowly triangular, attenuate; abaxial sclerenchyma a solid layer; ovary higher than wide, its

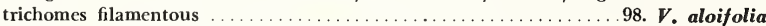

14. Caudex very short, simple, the plants essentially cespitose; blades vestite on both sides with spreading hairs, sublinear, acute; abaxial sclerenchyma scant and scattered; ovary globose or depressed-globose, its trichomes setiform ......99. V. metzgerae

13. Blades tomentose beneath with minute, crisp, tangled hairs; furrows papillate.

15. Tepals to $60 \mathrm{~mm}$ long; blades sparsely tomentose beneath, the nerves visible ......

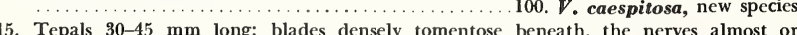
wholly invisible

101. V. glandulifera

\section{Section Radia}

1. Flowers appearing sessile, the scape covered by the leaf-sheaths or blade bases. Brazil: Minas Gerais

1. Flowers obviously scapose.

2. Leaf-blades wholly glabrous or vestite only on the margins and midrib or above the base.

3. Blade trichomes consisting of flat wholly sessile plates and only on the ventral surface at the base; translucent cells above both bundles and furrows, furrows papillate. 
Brazil: Minas Gerais

103. V. caput-ardeae, new species

3. Blade trichomes terete or if flat not sessile.

4. Ovary glands slenderly stipitate.

5. Scapes $3 \mathrm{~cm}$ long above the leaf-sheaths; leaf-blades $10 \mathrm{~mm}$ wide. Brazil: Minas Gerais.

104. V. maguirei

5. Scapes 9-14 cm long above the leaf-sheaths; leaf-blades $6-8 \mathrm{~mm}$ wide, glandularpunctate above, sparsely long-ciliate.

6. Branches including leaf-sheaths $16 \mathrm{~mm}$ thick; leaf-blades $6-8 \mathrm{~mm}$ wide, flat; scapes to $14 \mathrm{~cm}$ long above the leaf-sheaths; tepals reddish; stamens 12 . Colombia: Vaupés

105. V. maudeana

6. Branches including leaf-sheaths $8 \mathrm{~mm}$ thick; leaf-blades $6 \mathrm{~mm}$ wide, involute; translucent cells above both bundles and furrows; scape to $9 \mathrm{~cm}$ long. Brazil: Rio Branco 106. V. uleana

4. Ovary glands subsessile or very shortly and broadly stipitate; perianth-tube 70-80 mm long; leaf-blades appressed-short-ciliate at base; translucent cells above both bundles and furrows. Brazil: Goiás.

7. Flowers exceeding the leaves; leaf-sheaths spotted with dark brown; lateral

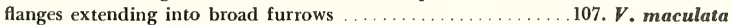

7. Flowers not exceeding the leaves; leaf-sheaths not spotted; no lateral flanges in very narrow furrows $\ldots \ldots \ldots \ldots \ldots \ldots \ldots \ldots \ldots \ldots \ldots \ldots \ldots \ldots \ldots$ 108. machrisiana

2. Leaf-blades generally and evenly vestite beneath, at least toward base.

8. Blades about equally vestite on both sides, but the trichomes of the upper side sometimes smaller.

9. Leaf-trichomes spreading, elongate; perianth-tube $50-65 \mathrm{~mm}$ long.

10. Leaf-sheaths broad, lanate. Brazil: Goiás, Distrito Federal ... 109. V. lanata

10. Leaf-sheaths narrow, glabrous. Brazil: Minas Gerais .......110. V. hirsuta

9. Leaf-trichomes erect.

11. Perianth-tube $70-80 \mathrm{~mm}$ long; leaf-blades to $17 \mathrm{~mm}$ wide; translucent cells above both bundles and furrows. Brazil: Goiás ..........111. V. dawsonii

11. Perianth-tube $45-55 \mathrm{~mm}$ long; leaf-blades not over $11 \mathrm{~mm}$ wide.

12. Scape $15 \mathrm{~cm}$ long; leaf-blades $8 \mathrm{~mm}$ wide; perianth-tube $45 \mathrm{~mm}$ long. Brazil: Goiás ...........................112. Velutinosa

12. Scape $8 \mathrm{~cm}$ long; leaf-blades $10-11 \mathrm{~mm}$ wide; perianth-tube $50-55 \mathrm{~mm}$ long. Colombia: Vaupés, Meta ...............113. V.phantasmagorica

8. Blades glabrous above or much more laxly vestite than beneath.

13. Stems long and slender, 6-10 mm thick including the leaf-sheaths; leaf-sheaths erect, remaining entire; blade: adaxial side ridged. Brazil: Goiás .114. V. annulata

13. Stems short or stout, to $\mathbf{4 0} \mathrm{mm}$ thick.

14. Mature leaf-blades not over $14 \mathrm{~cm}$ long; stems very short and stout. Brazil:

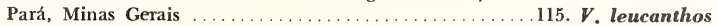

14. Mature leaf-blades much more than $14 \mathrm{~cm}$ long; stems elongate.

15. Scapes $2-7 \mathrm{~cm}$ long; trichomes of the ovary slender or tapered to the gland tip.

16. Mature leaf-blades to $25 \mathrm{~cm}$ long.

17. Scapes 4-6 cm long; perianth-tube to $50 \mathrm{~mm}$ long. Panama

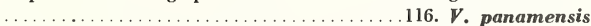

17. Scapes $2 \mathrm{~cm}$ long; perianth-tube to $65 \mathrm{~mm}$ long; leaf: no translucent cells above furrows. Brazil: Minas Gerais ............

117. $V$. riedeliana

16. Mature leaf-blades to $66 \mathrm{~cm}$ long; translucent cells above both bundles and furrows; scapes to $7 \mathrm{~cm}$ long; perianth-tube $50-55 \mathrm{~mm}$ long. Colombia: Vaupés ..................118. V. dumitiana

15. Scapes $9-18 \mathrm{~cm}$ long.

18. Perianth-tube $35-40 \mathrm{~mm}$ long; leaf: translucent cells above both bundles and furrows.

19. Leaf-blades to $20 \mathrm{~mm}$ wide. Colombia: Vaupés, Meta $\ldots \ldots \ldots \ldots \ldots \ldots \ldots \ldots \ldots \ldots \ldots \ldots \ldots \ldots \ldots \ldots \ldots \ldots$ 119. V. lithophila

19. Leaf-blades to $6 \mathrm{~mm}$ wide. Colombia: Meta

120. V. macarenensis

18. Perianth-tube $45-80 \mathrm{~mm}$ long. 
20. Scapes 9-11 cm long. Brazil: São Paulo ...121. V. rhynchocarpa

20. Scapes 12-18 cm long; leaf: translucent cells above both bundles and furrows. Guiana Highland of Venezuela and Guyana, Brazil: Goiás

122. V. tubiflora

1. Section Xerophytoides L. B. Smith \& Ayensu, new section

Foliorum lamina conduplicata ita teres, tepala libera, haud unguiculata, stamina 6 .

Leaf-blades subterete (conduplicate with each side semiterete); furrows absent or very small. Tepals free, not clawed. Stamens 6.

TYPE-SPECIEs.-Vellozia abietina Martius.

\section{Vellozia abietina Martius}

Vellozia abietina Martius, Nov. Gen. \& Sp., 1:14, pl. 6, 1823.Seubert in Martius, Fl. Bras., 3(1):74, 1847.-L. B. Smith, Contr. U.S. Nat. Herb., 35:259, 1962.-Ayensu, Proc. Biol. Soc. Washington, 85:471, fig. 1, 1972; Smithsonian Contr. Bot., 15:10, fig. 5a-b, pl. 24a-b, 1974.

Xerophyta abietina (Martius) Sprengel, Syst. Veg. 4: Cur. Post. 137, 1827.-Baker, Journ. Bot., 13:236, 1875.

TyPe.-Bare rocky summits, Itambé do Mato Dentro and Diamantina, Minas Gerais, Brazil, 900$1200 \mathrm{~m}$ alt, Martius s $n$ (M, holotype; F, photo 18967).

Distribution.-Brazil: Minas Gerais: Datas, Diamantina, Gouveia, Itambé, Serro.

\section{Vellozia tragacantha (Martius ex Schultes f.) Martius ex Seubert}

Figure 2

Vellozia tragacantha (Martius ex Schultes f.) Martius ex Seubert in Martius, Fl. Bras., 3(1):75, 1847.-L. B. Smith, Contr. U.S. Nat. Herb., 35:259, 1962.-Ayensu, Smithsonian Contr. Bot., 15:42, fig. 6a-b, 1974.

Xerophyta tragacantha Martius ex Schultes f. in Roemer \& Schultes Syst., 7:290, 1826.-Baker, Journ. Bot., 13:236, 1875.

TyPe.-Villa de Rio das Contas [Itacaré], Bahia, Brazil. [Oct 1818], Martius s $n$ (M, holotype; F, photo 18998).

Distribution.-Brazil: Bahia: Itacaré. Minas Gerais: Barão de Cocais, Curvelo, Gouveia.

\section{Vellozia scoparia Goethart \& Henrard}

\section{Figure 3}

Vellozia scoparia Goethart \& Henrard, Blumea, 2:380, 1937.L. B. Smith, Contr. U.S. Nat. Herb., 35:262, 1962.

Leaf Anatomy (L. B. Smith, Ayensu \& Hatschbach 16006).-Surface View: Hairs: few tufts present on abaxial surface. Epidermis: adaxial cells 4- to 7-sided; thin-walled. Abaxial cells mostly rectangular; thin-walled. Stomata: tetracytic, $21 \times 12 \mu \mathrm{m}$; present mainly on adaxial surface, arranged in linear rows.

Transverse Section of Lamina: Dorsiventral; V-shaped with deep median adaxial groove. Abaxial surface undulating, adaxial side highly ridged. Epidermis: cells on both surfaces rounded; thin-walled. Cuticle: thickened on both surfaces; thicker on abaxial; smooth. Stomata: present on adaxial surface only; associated with fairly large substomatal chamber. Mesophyll: 3 or 4 layers of compactly arranged palisade cells followed by 9 or 10 layers of closely packed, rounded spongy tissue. Vascular bundles: 8; commissural bundles observed. One to three large vessels present in each vascular bundle. Two phloem units lying lateral to xylem in short flanges of short, thick abaxial girder. Adaxial cap present on each vascular bundle. Bundle sheath completely surrounding each vascular bundle. No abaxial sclerenchyma strands or layers observed. Crystals: none observed. Tannins: present in adaxial epidermal cells and in bundle sheath cells; few in abaxial epidermal cells.

Note: The -presence of stomata on the adaxial surface with distinct substomatal chamber is very characteristic of this species.

TYPE.-In campo, Tombador near Diamantina, Minas Gerais, Brazil, 6 April 1892, Glaziou 19939 (L, holotype; B, photo).

Distribution.-Brazil: Minas Gerais: Forming mats in brown sandy soil, near Sopa, Guinda to São João da Chapada, 18 January 1959, Irwin 2489 (TEX, US). On rocks, $7 \mathrm{~km}$ west of Diamantina, 21 January 1972, L. B. Smith, Ayensu \& Hatschbach 

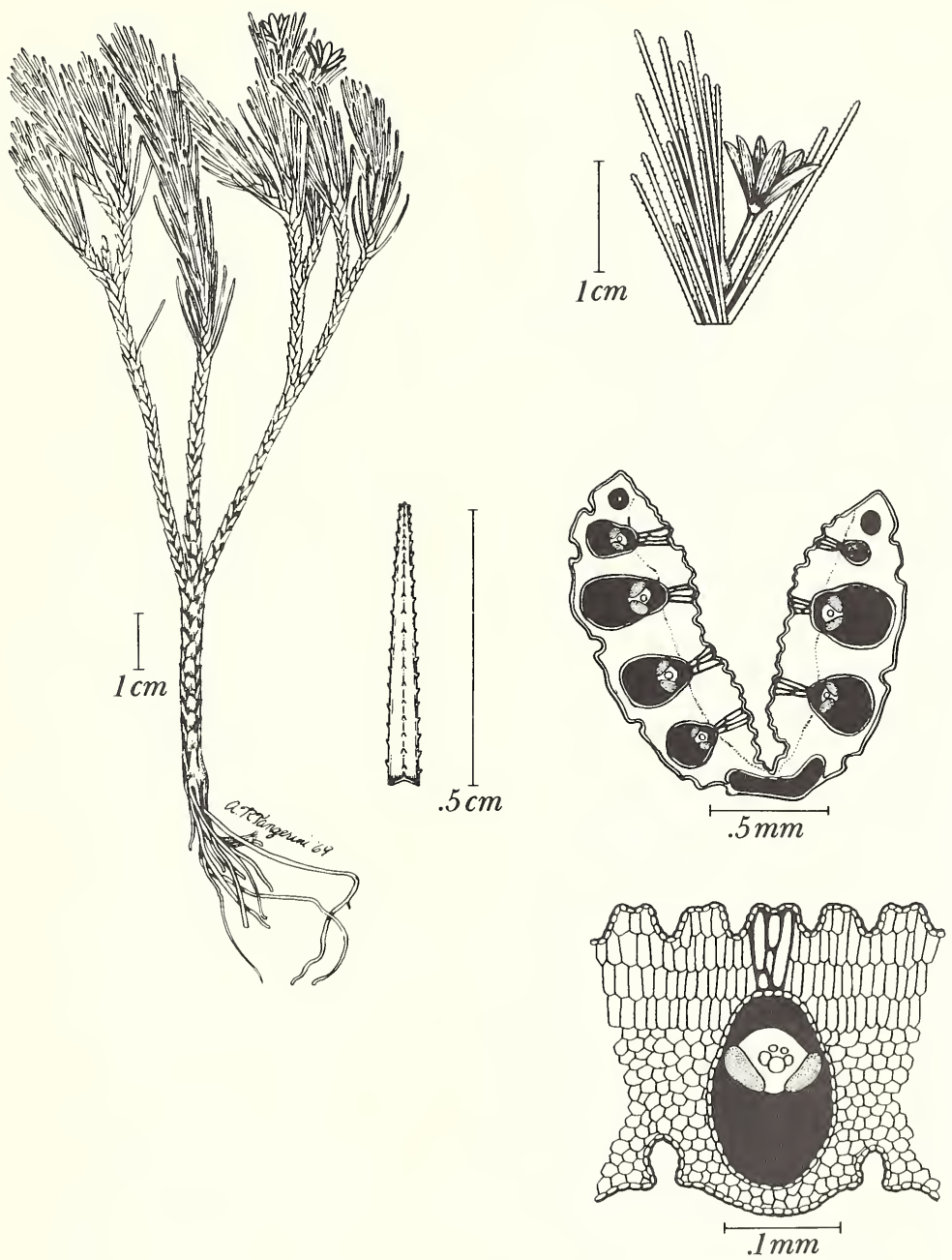

Figure 2.-Vellozia tragacantha (Martius ex Schultes f.) Martius ex Seubert (Claussen 13). 


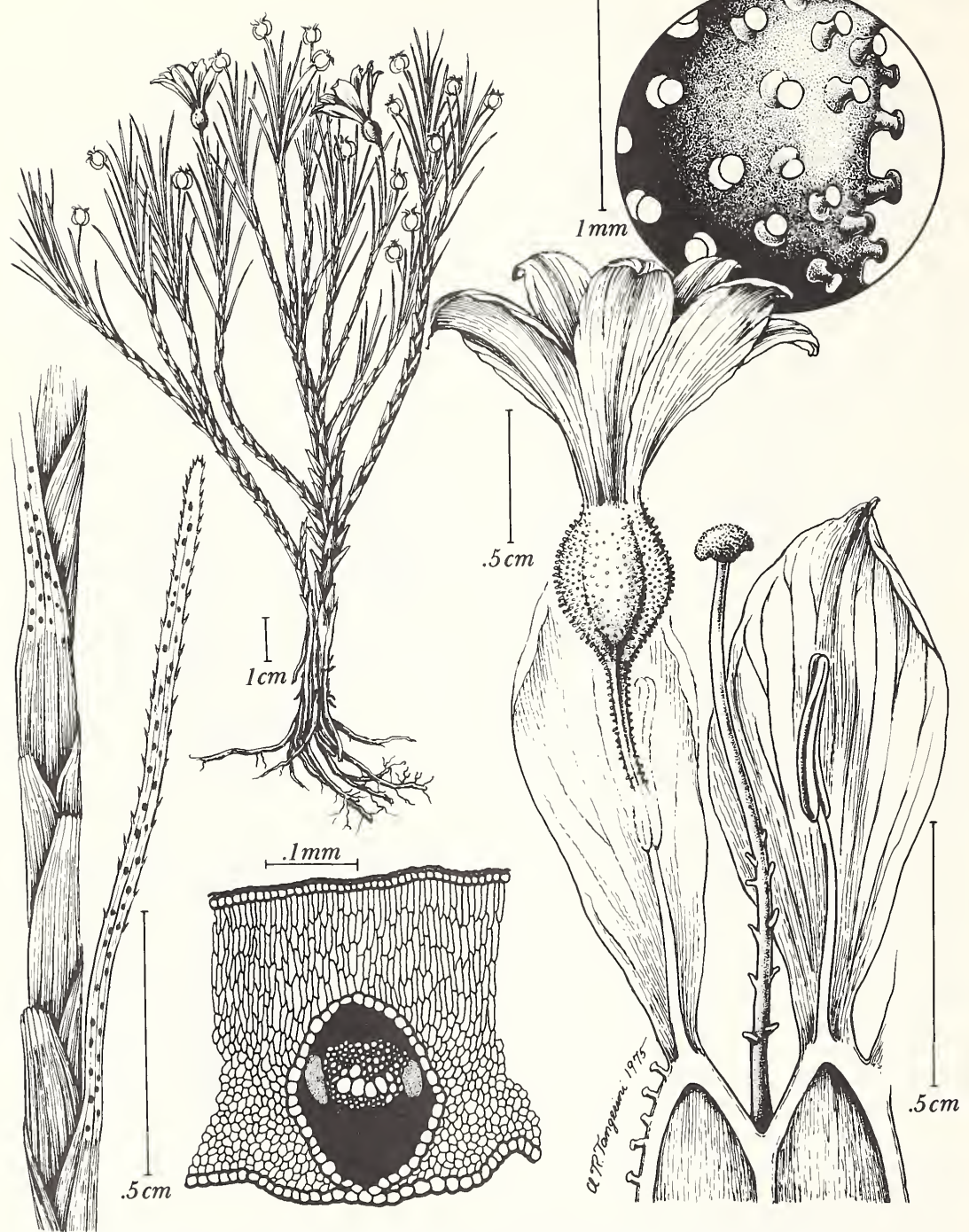

Figure 3.-Vellozia scoparia Goethart \& Henrard (L. B. Smith, Ayensu \& Hatschbach 16006, flower Irwin 2489). 
16006 (HB, US). Also from Jaboticatubas and São João de Chapada.

\section{Vellozia mimima Pohl}

Vellozia minima Pohl, Pl. Bras., 1:119, pl. 94, 1827.-Seubert in Martius, F1. Bras., 3(1):75, 1847.-L. B. Smith, Contr. U.S. Nat. Herb., 35:262, 1962.-Ayensu, Smithsonian Contr. Bot., 15:33, fig. 8d, pl. 15b, 1974.

Xerophyta minima (Pohl) Baker, Journ. Bot., 13:236, 1875. Vellozia pleurocarpa Goethart \& Henrard, Blumea, 2:369, 1937.-L. B. Smith, Contr. U.S. Nat. Herb., 35:262, 1962 [type: In campo, Biribiri, near Diamantina, Minas Gerais, Brazil, 25 March 1892, Glaziou 19941 (L, B photo)].

Type.-Dry mountains, Serra Itambé, Minas Gerais, Brazil, Pohl s $n$ (W, holotype lost; M, isotype; F, photo 18979).

Distribution.-Brazil: Minas Gerais: Diamantina, Jaboticatubas.

\section{Vellozia virgata Goethart \& Henrard}

Vellozia virgata Goethart \& Henrard, Blumea, 2:382, 1937.L. B. Smith, Contr. U.S. Nat. Herb., 35:261, 1962.-Ayensu, Smithsonian Contr. Bot., 15:45, fig. 3a-b, pl. 46d, 1974.

TYPE.-Rocky campo, Serra de São José (João?) del Rei, Minas Gerais, Brazil, 20 January 1889, Glaziou 17832 (L, holotype; B, photo).

Distribution.-Brazil: Minas Gerais: Pico Itabira do Campo, São João(?) del Rei.

\section{Section Vellozia}

Leaf blades more or less flattened; furrows almost always distinct on the abaxial side. Tepals, or at least their margins, free, usually without claws. Stamens rarely 6 , usually more than 6 .

Type-Species._Vellozia glabra Mikan.

\section{Subkey I}

\section{Vellozia ciliata L. B. Smith}

Vellozia ciliata L. B. Smith, Contr. U.S. Nat. Herb., 35:290, pl. 11: figs. 58, 59, 1962.-Ayensu, Smithsonian Contr. Bot., 15:15, pls. 5 c, $36 \mathrm{~b}, 49 \mathrm{a}-\mathrm{c}, 1974$.

TyPe.-On sandstone outcrop, ca. 3-5 km east of Serro, along road from Conceição to Diamantina, Minas Gerais, Brazil, 9 August 1960, Maguire,
Magalhães \& Maguire 49158 (US, holotype; NY, isotype).

Distribution.-Brazil: Minas Gerais: Diamantina, Serro.

\section{Vellozia taxifolia (Martius ex Schultes f.) Martius ex Seubert}

Vellozia taxifolia (Martius ex Schultes f.) Martius ex Seubert in Martius, F1. Bras., 3(1):75, 1847.-L. B. Smith, Contr. U.S. Nat. Herb., 35:262, 1962.-Ayensu, Smithsonian Contr. Bot., 15:39, fig. 8f, pl. 30a-c, 1974 .

Xerophyta taxifolia Martius ex Schultes f. in Roemer \&c Schultes, Syst., 7:291, 1826.-Baker, Journ. Bot., 13:236, 1875 .

TyPe.-Brazil, Martius s n (M, holotype; F, photo 18997).

Distrıbutıon.-Brazil: Minas Gerais: Conceição do Mato Dentro, Jaboticatubas.

\section{Vellozia cryptantha Seubert}

Vellozia cryptantha Seubert in Martius, F1. Bras., 3(1):80, 1847.-L. B. Smith, Contr. U.S. Nat. Herb., 35:263, 1962.

TyPE.-Minas Gerais, Brazil, Gardner 5229 (US, isotype).

Distribution.-Known only from the typecollection.

\section{Vellozia maxillarioides L. B. Smith}

Vellozia maxillarioides L. B. Smith, Contr. U.S. Nat. Herb., 35:285, pl. 11: fig. 37, 1962.

Type.-On sandstone, slopes and summit of Grão Mogul, Minas Gerais, Brazil, 900-1100 m alt, 17 August 1960, Maguire, Magalhães \& Maguire 49268 (US, holotype; NY, isotype).

Distribution.-Brazil: Minas Gerais: Grão Mogul.

10. Vellozia costata L. B. Smith \& Ayensu, new species

Figure 4

A $V$. incurvata Martius ex Schultes f., cui affinis, et a fere omnibus alteris, ovario glabro vel subglabro dense rotundato-costato differt.

Caudex to $5 \mathrm{dm}$ high, few-branched, the branches 


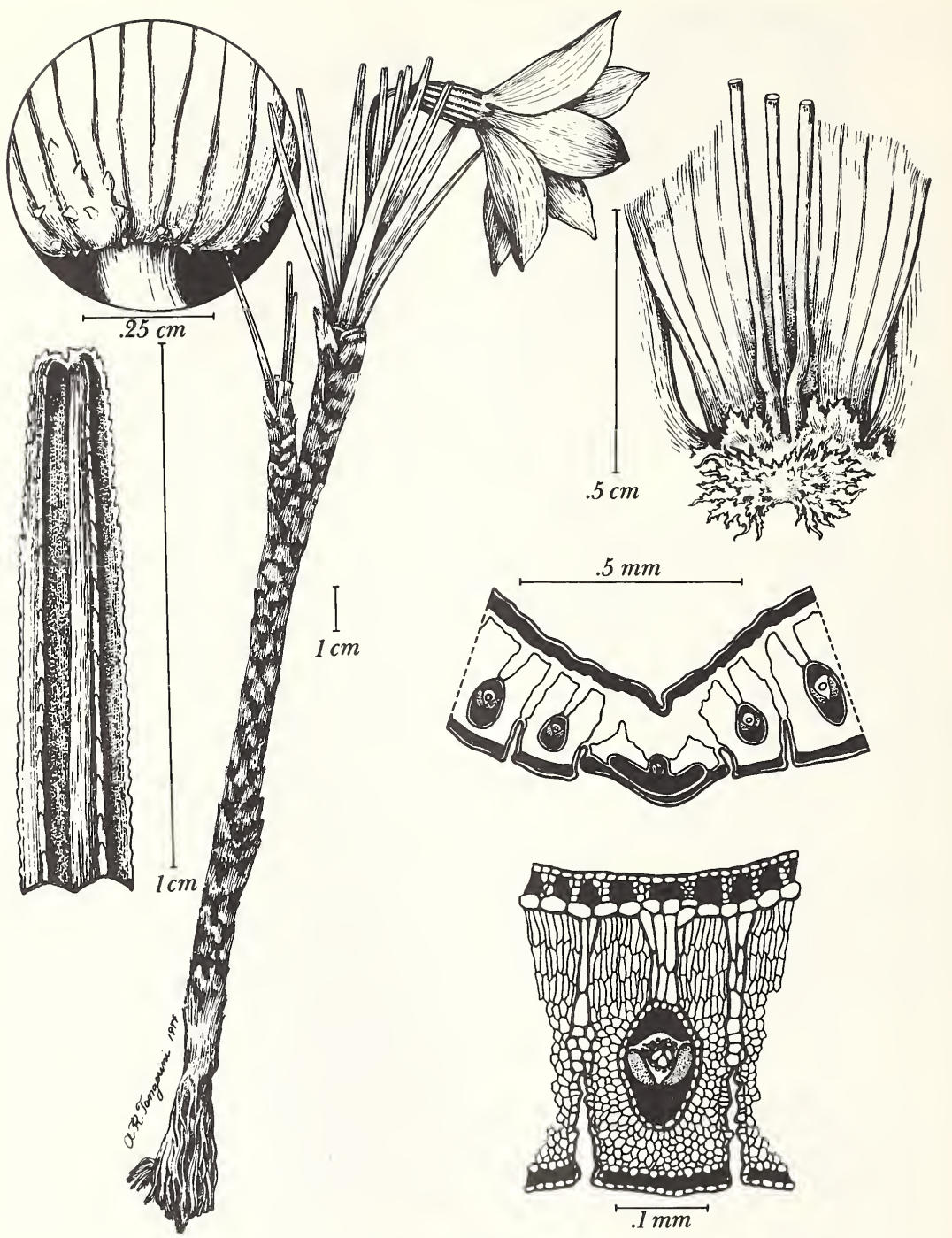

Figure 4.-Vellozia costata L. B. Smith \& Ayensu, new species (Irwin, Maxwell \& Wasshausen 20999). 
terete and 7-9 $\mathrm{mm}$ in diameter (including the old leaf-sheaths). Leaves densely 4- or 5-ranked; sheaths to $3 \mathrm{~cm}$ long, thin but remaining entire, densely nerved; blades about 10 in an apical fascicle, linear, retuse, to $6 \mathrm{~cm}$ long, $4.5 \mathrm{~mm}$ wide, densely nerved with the nerves prominent beneath, densely and finely serrate on the thickened margins and on the costa beneath, otherwise nearly even and glabrous.

Scapes solitary, mostly erect but decurved at apex, 3-6 cm long (above the leaf-sheaths), slightly over $1 \mathrm{~mm}$ in diameter, sulcate, glabrous. Ovary obovoid, $14 \mathrm{~mm}$ long, densely rounded-costate, smooth or very sparsely acicular at base. Tepals free, elliptic, $30 \mathrm{~mm}$ long, blue-violet (IIrwin). Stamens ca. 18, equal, the phalanges lacerate-appendaged; filaments $7 \mathrm{~mm}$ long, anthers linear, $10 \mathrm{~mm}$ long. Stigma peltate, broadly 3-lobed.

Leaf Anatomy.-Ayensu, Smithsonian Contr. Bot., 15:17, pl. 36c, 1974.

TYPE.-Rocky campo and outcrops, Serra do Espinhaço, ca. $17 \mathrm{~km}$ north of Serro on road (MG 2) to Diamantina, Minas Gerais, Brazil, $1200 \mathrm{~m}$ alt, 27 February 1968, Irwin, Maxwell of Wasshausen 20999 (US, holotype; NY, isotype).

Distribution.-Known only from the typecollection.

\section{Vellozia alata L. B. Smith}

Vellozia alata L. B. Smith, Contr. U.S. Nat. Herb., 35:260, pl. 1, 1962.-Ayensu, Smithsonian Contr. Bot., 15:10, fig. 5c-e, pl. 24c-e, 1974.

Tepals narrowly rhombic, clawed, acute, 60-105 $\mathrm{mm}$ long, lilac (IMenezes).

TyPE.-Some $31 / 2$ miles $(5.6 \mathrm{~km})$ from Hotel Chapeu do Sol, Serra do Cipó, Minas Gerais, Brazil, 19 December 1959, Maguire \& Murça Pires 44690 (US, holotype; NY, isotype).

Distribution.-Brazil: Minas Gerais: Jaboticatubas (Serra do Cipó).

\section{Vellozia sellovii Seubert}

\section{FigURE 5}

Vellozia sellovii Seubert in Martius, F1. Bras., 3(1):75, 1847.L. B. Smith, Contr. U.S. Nat. Herb., 35:259, 1962; Phytologia, 9:262, fig. 1, 2, 1963.-Ayensu, Smithsonian Contr. Bot., 15:38, pl. 46c, 1974.

Xerophyta sellovii (Seubert) Baker, Journ. Bot., 13:236, 1875 [as "Selloi"].
TyPe.-Without locality, Brazil, Sellow s $n$ (B, holotype; US, photo).

Distribution.-Brazil: Minas Gerais: Caeté, Serra da Piedade.

\section{Vellozia laevis L. B. Smith}

Vellozia laevis L. B. Smith, Phytologia, 8:507, figs. 1, 2, 1963.

TyPe.-Among rocks, Caraça, Minas Gerais, Brazil, 1824, Riedel s $n$ (K, holotype; US, photo).

Distribution.-Known only from the typecollection.

\section{Vellozia macedonis Woodson}

Vellozia macedonis Woodson, Ann. Mo. Bot. Gard., 37:398, 1950.

TyPE.-Campo of the "Canga", Saramenha, Ouro Preto, Minas Gerais, Brazil, 7 January 1950, Macedo 2072 (MO, holotype; US, isotype).

Distribution.-Brazil: Minas Gerais: Caraça, Ouro Preto.

\section{Vellozia tenella Martius ex Schultes $\mathrm{f}$.}

Vellozia tenella Martius ex Schultes f. in Roemer \& Schultes, Syst., 7:293, 1826.-L. B. Smith, Contr. U.S. Nat. Herb., 35:259, 1962.-Ayensu, Smithsonian Contr. Bot., 15:39, pl. 44d, 1974.

Vellozia graminea Pohl, Pl. Bras., 1:118, pl. 93, 1827--Seubert in Martius, Fl. Bras., 3(1):76, pl. 9, 1847 [type: dry mountains, Inficionado and Villa Rica (Ouro Preto), Minas Gerais, Brazil (W, holotype lost; M, isotype; F, photo 18976)].

TyPE.-Minas Gerais, Brazil, Ackermann $s n$, Claussen s $n$ (syntypes n. v.).

Distribution.-Brazil: Minas Gerais: Caraça, Serra do Itabirito, Ouro Preto.

\section{Vellozia glabra Mikan}

Vellozia glabra Mikan, Delect. Fl. \& Faun. Brasil. fasc. 2, 1820.-Sprengel, Syst. Veg., 3:338, 1826.-Schultes f. in Roemer \& Schultes, Syst., 7:293, 1826.-L. B. Smith, Contr. U.S. Nat. Herb., 35:260, 1962.-Ayensu, Smithsonian Contr. Bot., 15:24, pls. 24f-g, 25a, 1974.-L. B. Smith, Taxon, 24(4):474, 1975.

Vellozia capsulis scapisque glabris Vandelli, Fl. Lusit. \& Brasil. Spec., 33, pl. 2, 1788.

Vellozia phalocarpa Pohl, Pl. Brasil., 1:123, pl. 98, 1827.Seubert in Martius, Fl. Bras., 3(1):76, 1847 [type: in stands, 

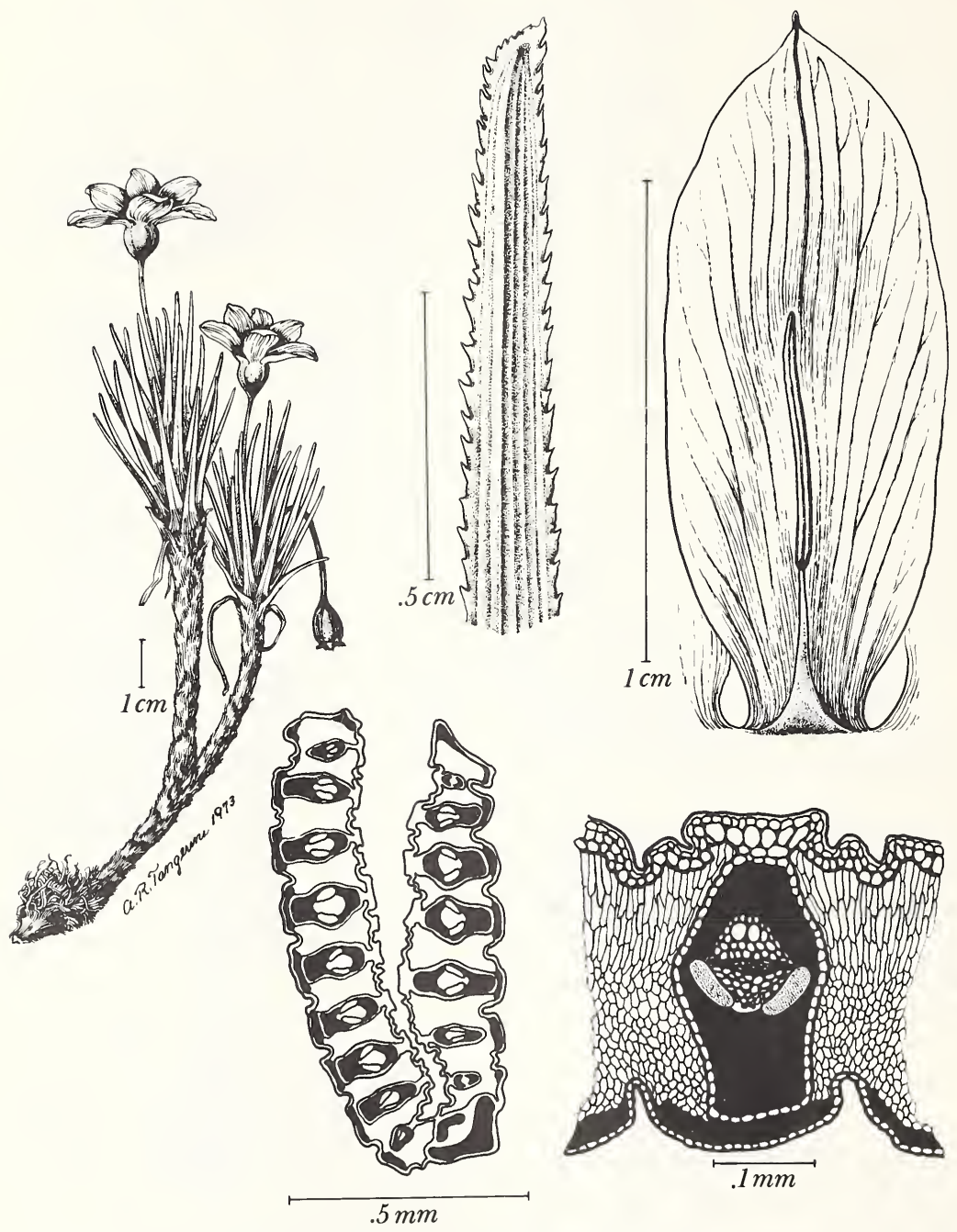

Figure 5.-Vellozia sellovii Seubert (Sellow $s$ ). 
dry mountains, Serra de São Felis, Goiás, Brazil, October 1819, Pohls $n$ (W n v)].

TYPE.-Description and illustration. No specimen known.

Distribution.-Brazil: Minas Gerais: Jaboticatubas.

\section{Vellozia pusilla Pohl}

Vellozia pusilla Pohl, Pl. Brasil., 1:122, pl. 97, 1827.-Seubert in Martius, F1. Bras., 3(1):76, 1847.-L. B. Smith, Contr. U.S. Nat. Herb., 35:259, 1962.

Type.-Sandy and gravelly summit of Pico de Itambé, Minas Gerais, Brazil, November 1820, Pohl $s n$ (W, holotype lost).

Distribution.-Brazil: Minas Gerais: Cerro do Cipó south of Conceição do Mato Dentro, Itambé do Mato Dentro.

\section{Vellozia incurvata Martius ex Schultes f.}

Vellozia incurvata Martius ex Schultes f. in Roemer \& Schultes, Syst., 7:293, 1826.-Seubert in Martius, Fl. Bras., 3(1):76, 1847.-L. B. Smith, Contr. U.S. Nat. Herb., 35:260, 1962.-Ayensu, Smithsonian Contr. Bot., 15:29, pl. 42f, 1974.

?Vellozia aloifolia sensu Gaudichaud, Atl. Bonite, pl. 127, 1852 [non Martius, 1823; based on Gaudichaud s $n$ (P n v)].

TyPE.-Minas Gerais, Brazil, Martius s $n$ (M, holotype; F, photo 18977).

Distribution.-Brazil: Minas Gerais: Serra de Ouro Branco, also Mun. Gouvêa, Lapinha, Presidente Juscelino.

\section{Vellozia brachypoda L. B. Smith \& Ayensu, new species}

Figure 6

A $V$. variabile Martius ex Schultes f., cui versimiliter affinis, ovario omnino tereti differt.

Caudex simple, 2-5 cm long, 20-25 $\mathrm{mm}$ in diameter including the persistent leaf-sheaths. Leaves about 8 in each season; sheaths very dense with almost no exposure, glabrous, soon dividing into coarse fibers; blades reflexing, persistent, linear, attenuate to a fine apex, ca. $15 \mathrm{~cm}$ long, $5 \mathrm{~mm}$ wide, flat or somewhat inrolled, glabrous except for the finely setose-serrulate margins.

Scape terminal, solitary, $15 \mathrm{~mm}$ long above the leaf-sheaths, terete, sulcate, glabrous. Ovary subcylindric, 8-10 $\mathrm{mm}$ long, glabrous or very obscurely stipitate-glandular; epigynous tube subcylindric, 4 $\mathrm{mm}$ high. Tepals free, oblanceolate, $15 \mathrm{~mm}$ long, violet (IIrwin), glabrous. Stamens ca. 40-50, imperfectly known. Stigmas horizontal, suborbicular.

Leaf Anatomy.-Ayensu, Smithsonian Contr. Bot., 15:13, pl. 42b, 1974.

TYPE.-Common on rock outcrops, bordering creek, ca. 15 km west of Grão Mogul, Minas Gerais, Brazil, $950 \mathrm{~m}$ alt, 19 February 1969, Irwin, Reis dos Santos, Souza \& Fonseca 23534 (US, holotype; NY, isotype).

Distribution.-Known only from the typelocality.

\section{Vellozia sincorana $\mathrm{L}$. B. Smith \& Ayensu, new species}

\section{Figure 7}

V. variabile Martius ex Schultes f. in systemate nostro proxima sed ovario tereti elongato, petalis magnis basi longe attenuatis, phalangis oblongis nudis differt.

Caudex 1-4 m high (!Ule). Leaf-blades probably persistent (because strongly curved at base), $45 \mathrm{~cm}$ long, $20 \mathrm{~mm}$ wide, very narrowly triangular, finely attenuate, flat, smooth and glabrous except for the laxly and obscurely serrulate margins.

Scape (only 1 known) at least $7 \mathrm{~cm}$ long, $3 \mathrm{~mm}$ in diameter, terete, nearly even, glabrous. Ovary slenderly fusiform, terete, $4 \mathrm{~cm}$ long, $8 \mathrm{~mm}$ in diameter, even, glabrous; epigynous tube subcylindric, $7 \mathrm{~mm}$ high. Tepals free, oblanceolate, broadly rounded and apiculate, $7 \mathrm{~cm}$ long. Stamens ca. 50, much shorter than the tepals; anthers long, scarcely broader than the filaments; phalanges oblong, unappendaged. Stigmas horizontal, suborbicular.

Leaf Anatomy (Ule 7098).-Surface View: Hairs: none observed. Epidermis: cells on both surfaces square to rectangular; thin-walled. Stomata: tetracytic, $18 \times 9 \mu \mathrm{m}$; most in abaxial furrows; few on abaxial surface.

Transverse Section of Lamina: Dorsiventral; widely V-shaped with deep and wide adaxial groove. Adaxial surface ridged; abaxial surface furrowed 1/4 thickness of blade. Epidermis: cells rounded to dome-shaped on both surfaces; few square on adaxial surface; thin-walled. Few adaxial 


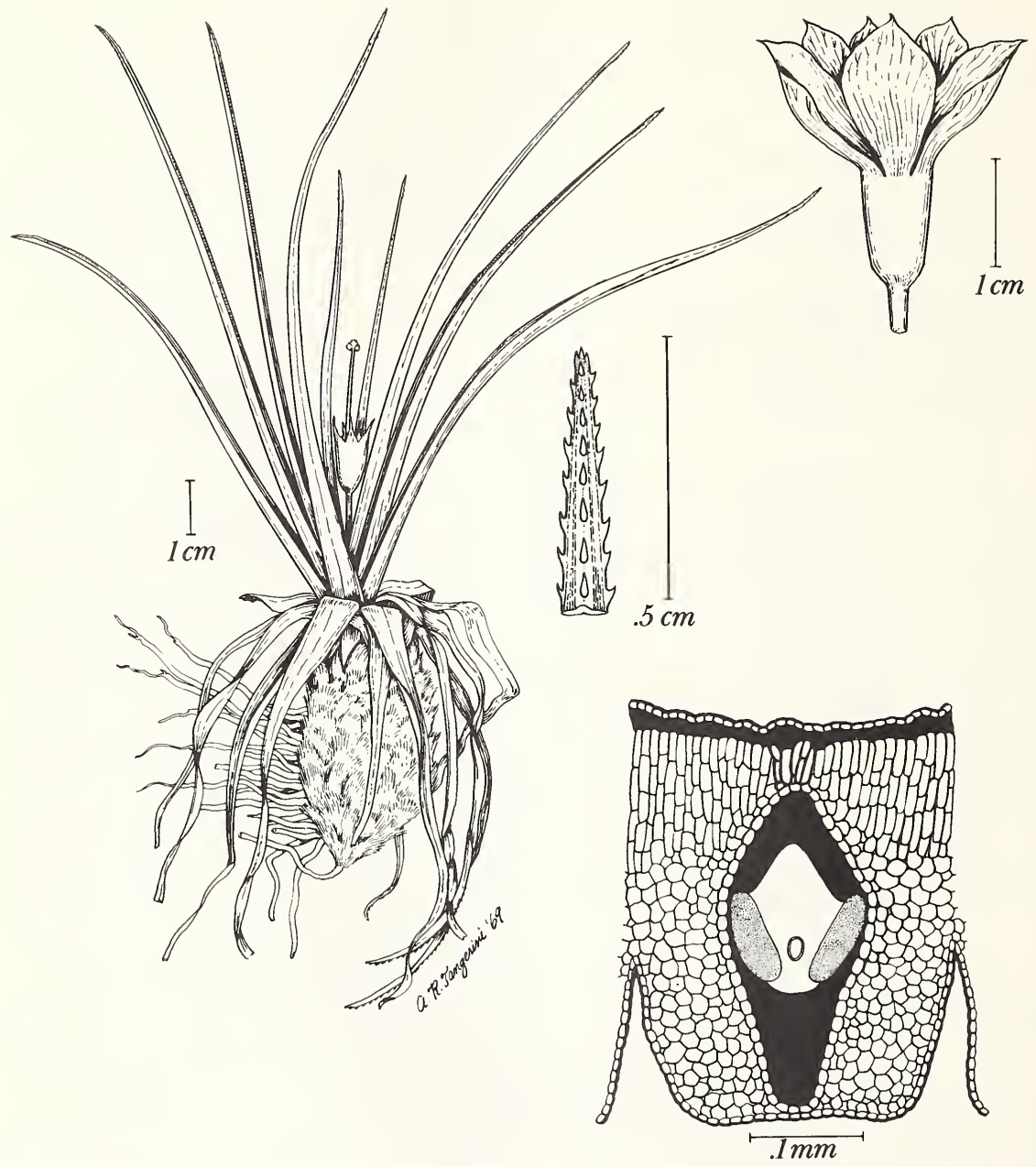

Figure 6.-Vellozia brachypoda L. B. Smith \& Ayensu, new species (Irwin, Reis dos Santos, Souza \& Fonseca 23534). 


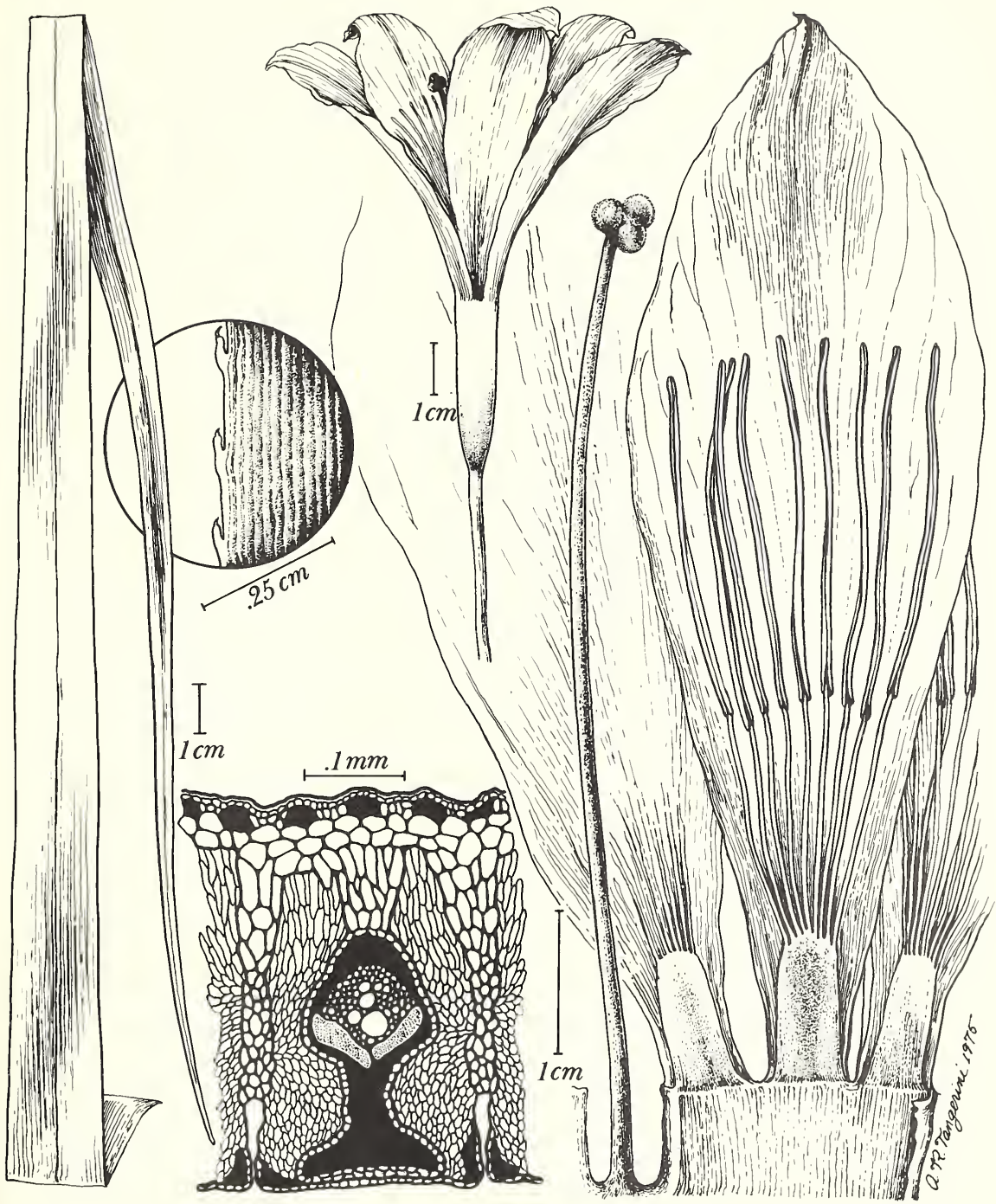

Figure 7.-Vellozia sincorana L. B. Smith \& Ayensu, new species (Ule 7098). 
cells replaced with sclerenchyma fibers. Subjacent to adaxial epidermis is a layer of thin-walled, rounded cells interspersed with sclerenchyma fiber strands; below this is 2 or 3 layers of large, thinwalled parenchyma cells. Subjacent to abaxial epidermis is a layer of thin-walled parenchyma cells. Cuticle: thickened and slightly ridged on both surfaces. Stomata: present in abaxial furrows; stomata flush with epidermal surface; substomatal chamber present. Mesophyll: 2 or 3 layers of palisade cells followed by compactly arranged spongy cells. Two or three layers of translucent cells radially arranged above vascular bundles and furrows. Sclerenchyma fiber bundles present at abaxial corners of furrows. Vascular bundles: 84; commissural bundles not observed. One-three large vessels present in each bundle, mostly one. Two phloem units lying laterally in flanges of abaxial girder; girder $Y$-shaped at top with sclerenchyma spreading laterally at bottom. Adaxial cap present on each bundle. Bundle sheath surrounding each bundle. Crystals: few present, especially in bundle sheath. Tannins: few present.

TYPE.-Serra do Sincorá, Bahia, Brazil. November 1906, Ule 7098 (L, holotype; US, photo).

Distribution.-Known only from the typecollection.

Note: Admittedly the position of Vellozia sincorana in our key does not express its true relationship. Probably it is much nearer $V$. punctulata Seubert and $V$. hatschbachii Smith \& Ayensu, new species.

\section{Vellozia verruculosa Martius ex Schultes $\mathrm{f}$.}

Vellozia verruculosa Martius ex Schultes f. in Roemer \& Schultes, Syst., 7:293, 1826.-Seubert in Martius, Fl. Bras., 3(1):77, 1847.-L. B. Smith, Contr. U.S. Nat. Herb., 35:260, 1962.-Ayensu, Smithsonian Contr. Bot., 15:44, pls. 19a-b, $26 a-c, 1974$.

TyPe.-Brazil, Goiás, Pohl s n (hb?, paratype); Mato Grosso, Manso s $n$ (hb?, paratype).

Distribution.-Brazil: Goiás: Corumba. Mato Grosso: without locality.

\section{Vellozia fibrosa Goethart \& Henrard}

\section{Figure 8}

Vellozia fibrosa Goethart \& Henrard, Blumea, 2:370, 1937.-
L. B. Smith, Contr. U.S. Nat. Herb., 35:260, 1962.-Ayensu, Smithsonian Contr. Bot., 15:23, pls. 17b, 26d-f, 1974.

TYPE.-Biribiri near Diamantina, Minas Gerais, Brazil, 30 March 1892, Glaziou 19936 (L, holotype; $\mathrm{B}$, photo; $\mathrm{P}$, isotype).

Distribution.-Brazil: Minas Gerais: Belo Horizonte(?), Diamantina.

\section{Vellozia crispata L. B. Smith}

Vellozia crispata L. B. Smith, Phytologia, 8:511, figs. 3-5, 1963.

TYPE.- “Environs de Rio de Janeiro et d'Ouro Preto" (most probably the latter), Minas Gerais, Brazil, 1883-84, Glaziou 15501 (K, holotype).

Distribution.-Known only from the typecollection.

\section{Vellozia punctulata Seubert}

Figure 9

Vellozia punctulata Seubert in Martius, F1. Bras., 3(1):82, 1847.-L. B. Smith, Contr. U.S. Nat. Herb., 35:264, 1962.Ayensu, Smithsonian Contr. Bot., 15:37, fig. 4a, pl. 44c, 1974.

TYPE.- "Mediterraneis" (probably the vicinity of São Salvador), Bahia, Brazil, Blanchet 2561 (G, holotype(?); F, photo 25079).

Distribution.-Known only from the typecollection.

\section{Vellozia hatschbachii L. B. Smith \& Ayensu, new species}

Figure 10

A $V$. punctulata Seubert, cui affinis, ovario tereti, tubo epigyno magno differt.

Caudex simple, erect, $1.2 \mathrm{~m}$ high, ca. $15 \mathrm{~mm}$ in diameter (without the leaf-sheaths). Leaves manyranked, very glutinous; sheaths very densely imbricate with almost no apical exposure; blades soon reflexed, long-persistent, very narrowly triangular, evenly long-attenuate, to $28 \mathrm{~cm}$ long (above the leaf-sheaths), $8 \mathrm{~mm}$ wide, flat, laxly serrulate, otherwise even and glabrous.

Scapes terminal, 1-3, erect, $3 \mathrm{~cm}$ long (above the leaf-sheaths), $1.2 \mathrm{~mm}$ in diameter, sulcate, glabrous. 


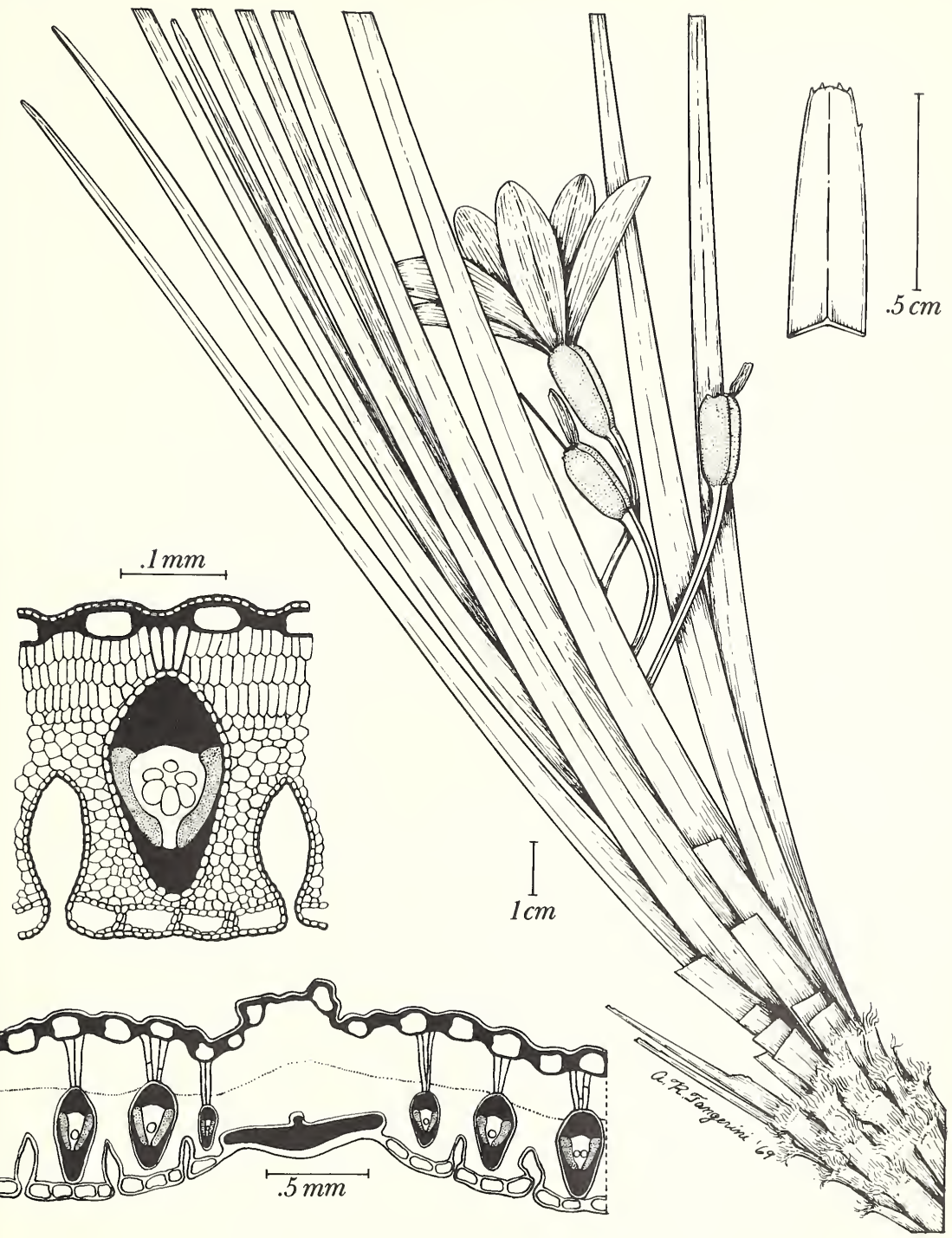

Figure 8.-Vellozia fibrosa Goethart \& Henrard (Glaziou 19936). 

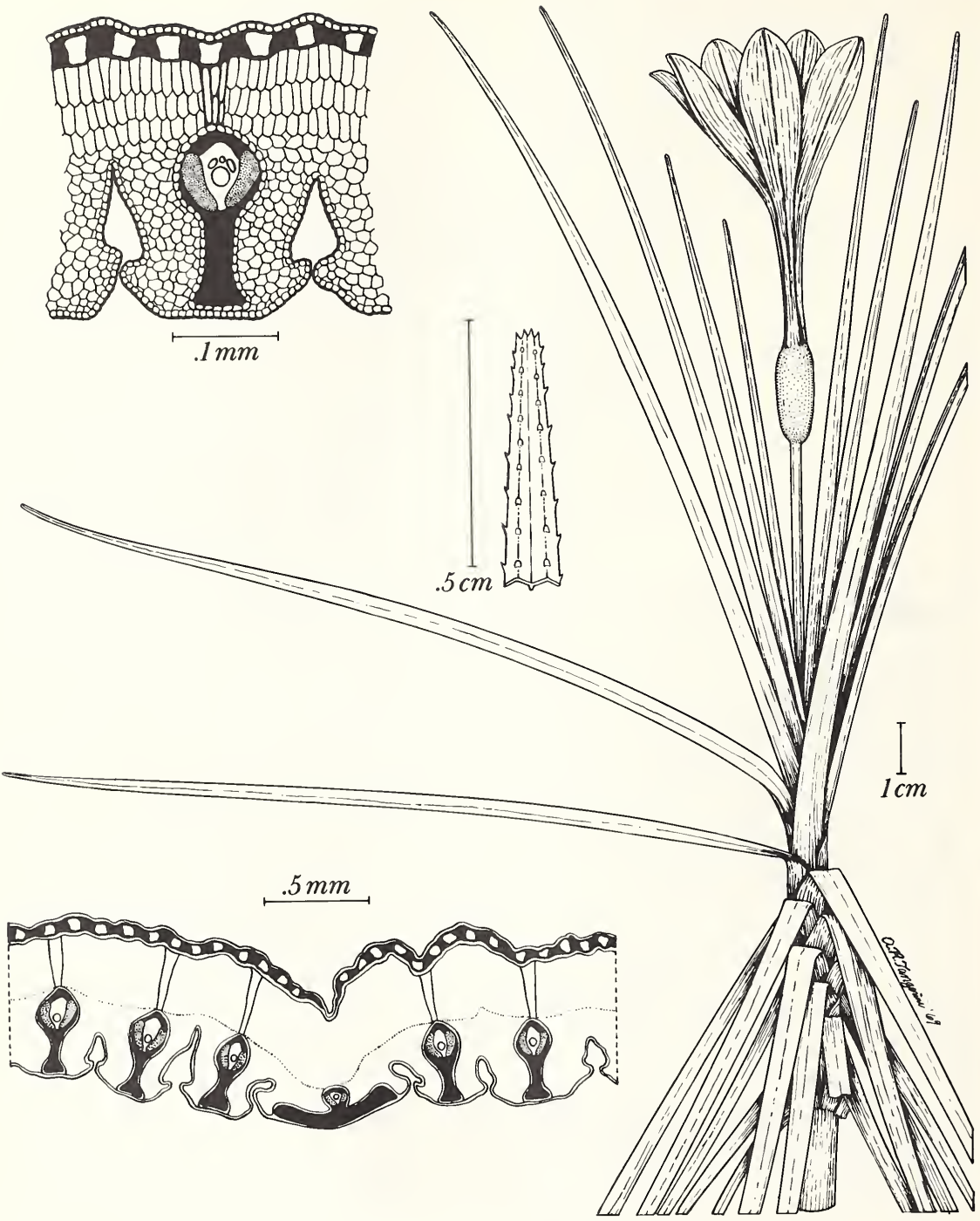

Figure 9.-Vellozia punctulata Seubert (Blanchet 2561). 


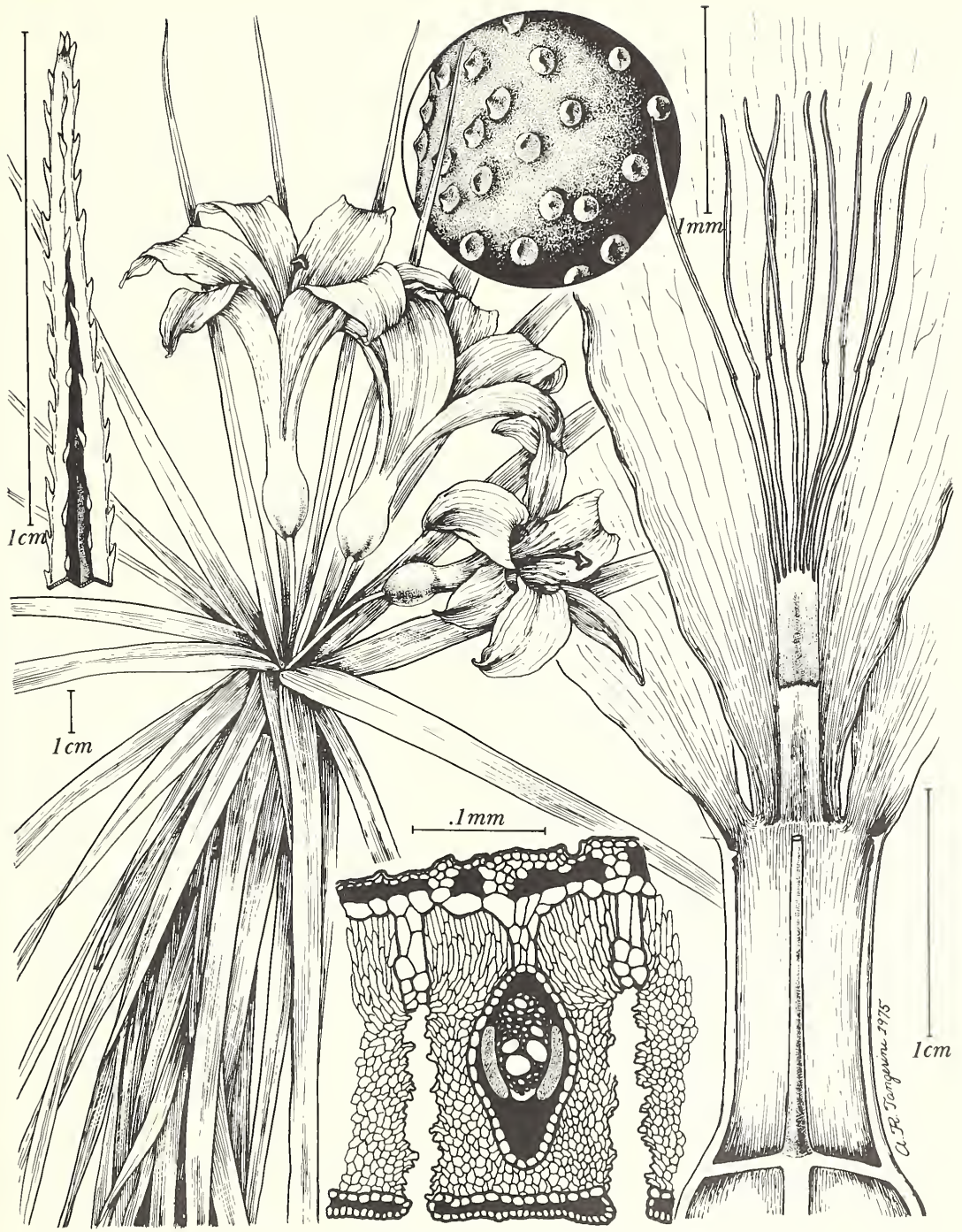

Figure 10.-Vellozia hatschbachii L. B. Smith \& Ayensu, new species (L. B. Smith, Ayensu \& Hatschbach 16002). 
Ovary broadly ellipsoid, $17 \mathrm{~mm}$ long, laxly scabrous, yellow; epigynous tube broadly subcylindric flaring at base, $8 \mathrm{~mm}$ long. Tepals spatulate, $6 \mathrm{~cm}$ long, white. Stamens ca. $36,4 \mathrm{~cm}$ long including the narrow unappendaged $25 \mathrm{~mm}$ long phalange. Style exceeding the stamens; stigmas terminal, orbicular, reflexed.

Leaf Anatomy.-Ayensu, Smithsonian Contr. Bot., 15:26, pls. 10a-b, 37a, 1974.

TYPE.-On rock dome, $33 \mathrm{~km}$ northeast of Diamantina, Mendanha, Mun. Cunha Magalhães, Minas Gerais, Brazil, 20 January 1972, L. B. Smith, Ayensu \& Hatschbach (separate no. 29009) 16002 (US, holotype; MBM, isotype).

Distribution.-Known only from the typecollection.

\section{Vellozia asperula Martius}

Vellozia asperula Martius, Nov. Gen. \& Sp., 1:15, pl. 8, 1823. -Seubert in Martius, Fl. Bras., 3(1):81, 1847.-L. B. Smith, Contr. U.S. Nat. Herb., 35:263, 1962.-Ayensu, Smithsonian Contr. Bot., 15:12, fig. 9d-f, 1974.

26a. Vellozia asperula var. asperula

Vellozia papillosa Pohl, Pl. Bras., 1:128, 1827 [type: sandy soil, Pico de Itambé, Minas Gerais, Brazil, Nov 1820, Pohl $s n$ (W, holotype lost)].

Leaf-blades $12-20 \mathrm{~cm}$ long, about equaling the flowers to somewhat exceeding them, $3-4 \mathrm{~mm}$ wide.

TyPE.-High rocky campo, Tres Barras (Rancho near Villa do Principe = Milho Verde?) and Tejuco (Diamantina), Minas Gerais, Brazil, $900 \mathrm{~m}$ alt, Martius s $n$ (M, holotype $\mathrm{n}$ v).

Distribution.-Brazil: Minas Gerais: Diamantina and vicinity, Itambé.

26b. Vellozia asperula var. filifolia $\mathrm{L}$. B. Smith

Vellozia asperula var. filifolia L. B. Smith, Contr. U.S. Nat. Herb., 35:263, 1962.

Leaf-blades over $30 \mathrm{~cm}$ long, much exceeding the flowers, only $1 \mathrm{~mm}$ wide.

TyPe.-Serra do Caraça, Minas Gerais, Brazil, 18 January 1921, Hoehne s $n$ (NY, holotype; SP no. 5025 , isotype).
Distribution.-Known only from the typecollection.

\section{Vellozia bradei Schulze-Menz ex Markgraf}

Vellozia bradei Schulze-Menz ex Markgraf, Notizblatt, 15:215, 1940.-L. B. Smith, Contr. U.S. Nat. Herb., 35:264, 1962.

TyPE.-On argillite, mountains east of Montes Claros, Serra Geral north of Grão Mogul, $1050 \mathrm{~m}$ alt, 12 November 1938, Markgraf, Brade o Mello Barreto 3512 (B, holotype; RB, photo).

Distribution.-Known only from the typecollection.

28. Vellozia ornata Martius ex Schultes f.

Vellozia ornata Martius ex Schultes f. in Roemer \& Schultes, Syst., 7:293, 1826.-Seubert in Martius, Fl. Bras., 3(1):80, 1847.-L. B. Smith, Contr. U.S. Nat. Herb., 35:264, 1962.Ayensu, Smithsonian Contr. Bot., 15:34, pl. 37f, 1974.

TyPe.-Minas Gerais, Brazil, Martius s $n$ (M, holotype; F, photo 18980).

Distribution.-Brazil: Minas Gerais: Cunha Magalhães, Diamantina, Gouveia.

\section{Vellozia granulata Goethart \& Henrard}

Figure 11

Vellozia granulata Goethart \& Henrard, Blumea, 2:373, 1937. -L. B. Smith, Contr. U.S. Nat. Herb., 35:264, 1962.Ayensu, Smithsonian Contr. Bot., 15:26, fig. 6c-e, 1974.

TYPE.-In campo, Diamantina, 14 April 1892, Glaziou 19934 (L, holotype; B, photo; P, isotype).

Distribution.-Brazil: Minas Gerais: Diamantina and vicinity.

\section{Vellozia piresiana L. B. Smith}

Vellozia piresiana L. B. Smith, Contr. U.S. Nat. Herb., 35:264, pl. 9: figs. 6, 7, 1962.-Ayensu, Smithsonian Contr. Bot., 15:35, pl. 43f, 1974.

Type.-Serra do Cipó at km 132, Minas Gerais, Brazil, 4 April 1951, Black \& Pires 51-12157 (INPA, holotype; US, photo).

Distribution.-Brazil: Minas Gerais: km 119 to 132 between Lagoa Santa and Conceição do Mato Dentro. 

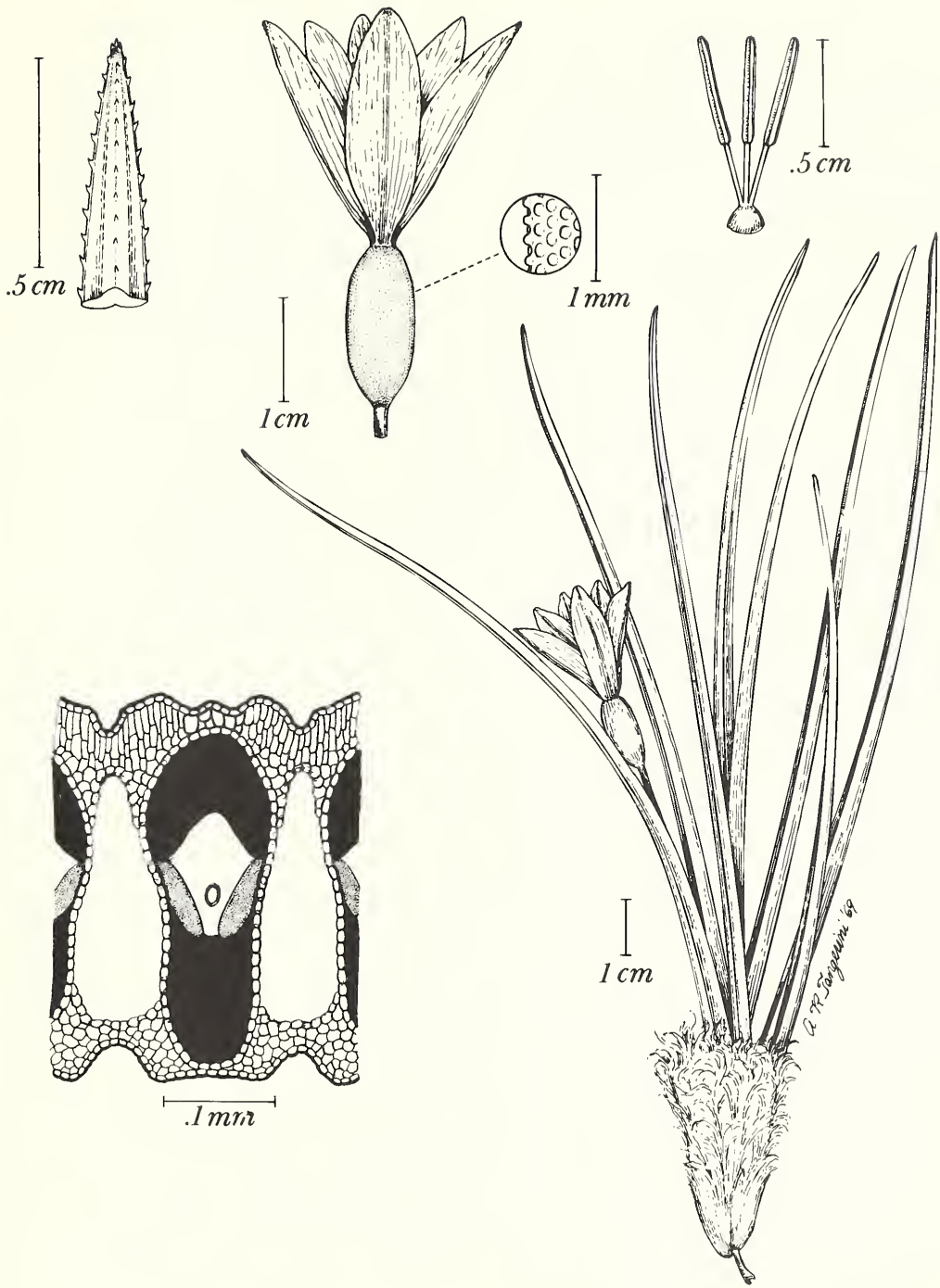

FiGURE 11.-Vellozia granulata Goethart \& Henrard (Glaziou 19934). 


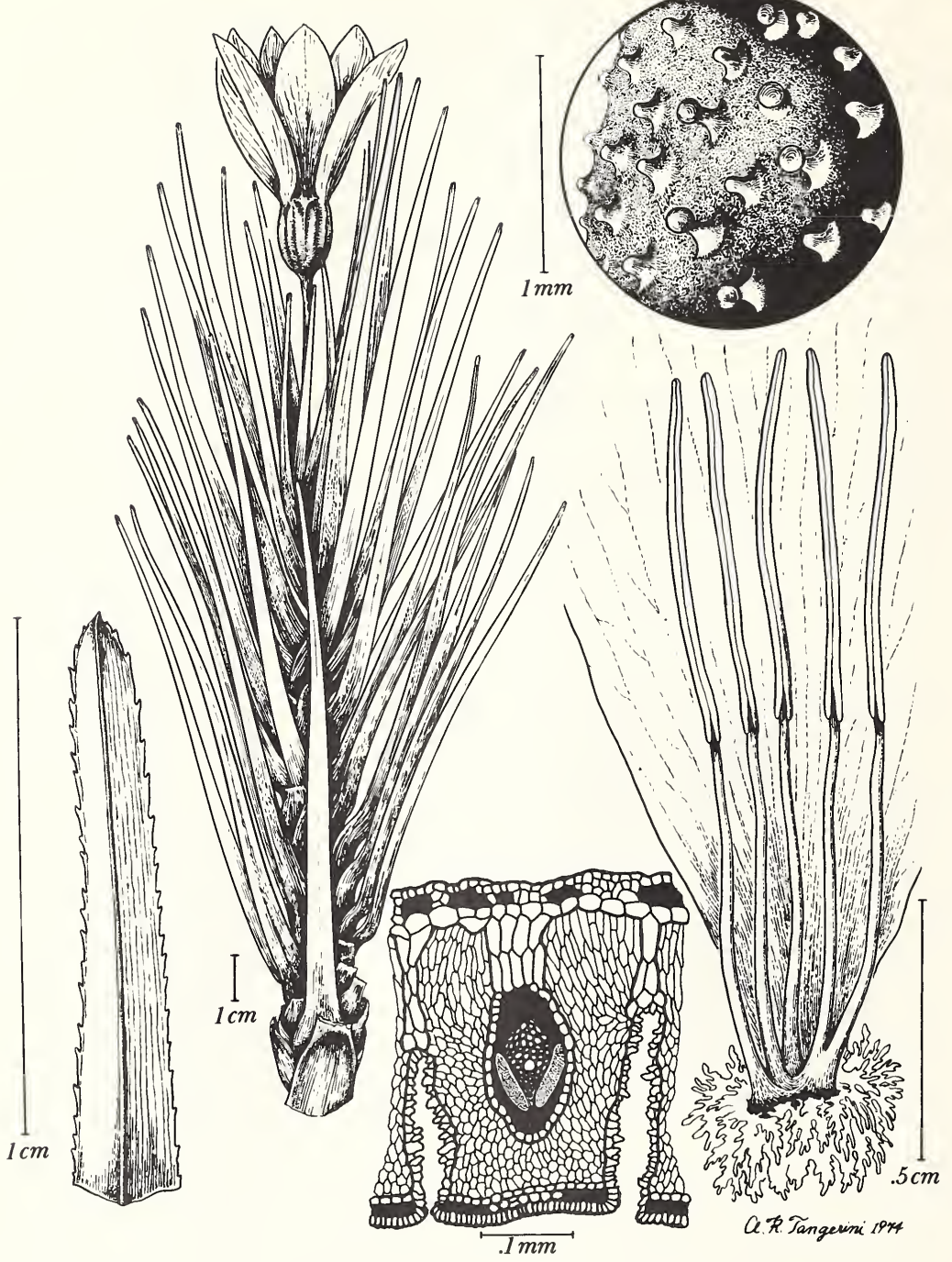

Figure 12.-Vellozia castanea L. B. Smith \& Ayensu, new species (Hoehne in SP 12495). 
31. Vellozia castanea L. B. Smith \& Ayensu, new species

Figure 12

A $V$. ramosissima L. B. Smith, cui affinis, foliorum vaginis parum exsertis et apice atro castaneis, laminis persistentibus erectisque differt.

Caudex (incompletely known) over $10 \mathrm{~cm}$ long, $15 \mathrm{~mm}$ in diameter including the leaf-sheaths. Leaves about 15-ranked; sheaths subdense with the dark castaneous sublustrous apices exposed for 5-10 $\mathrm{mm}$; blades persistent but erect, linear-triangular, obtuse, $13 \mathrm{~cm}$ long, $7 \mathrm{~mm}$ wide at base, revolute when dry, even and glabrous except for the obscurely serrulate margins.

Scape terminal, solitary, erect, much shorter than the leaves, laxly and minutely pale-glandular. Ovary ellipsoid, obtusely angled, $15 \mathrm{~mm}$ long, laxly and evenly vestite with minute pale stipitate glands, epigynous tube broad ca. $3 \mathrm{~mm}$ high. Tepals free, elliptic, $35 \mathrm{~mm}$ long. Stamens ca. 18, included; phalanges with lacerate appendages.

LeAf ANATOMY (Hoehne in SP 12495).-Surface View: Hairs: none observed. Epidermis: cells on both surfaces square to rectangular; thin-walled. Stomata: tetracytic; $18 \times 12 \mu \mathrm{m}$; present mostly in abaxial furrows; few on abaxial surface.

Transverse Section of Lamina: Dorsiventral; widely V-shaped with small median adaxial groove. Adaxial surface ridged; abaxial surface furrowed $1 / 2$ thickness of blade. Epidermis: cells on both surfaces rounded to dome-shaped; few conical; thin-walled. Subjacent to adaxial epidermis is 2 or 3 layers of thin-walled parenchyma heavily interspersed with sclerenchyma fiber bundles. Below this is a layer of large, thin-walled parenchyma cells. Subjacent to abaxial epidermis is 1 or 2 layers of thin-walled parenchyma interspersed with sclerenchyma fiber bundles; below is a layer of thin-walled parenchyma. Cuticle: slightly thickened and ridged on both surfaces. Stomata: mostly present in abaxial furrows; few on abaxial surface; stomata flush with epidermal surface; small substomatal chamber present; protected by few projections from walls of furrows. Mesophyll: 3 or 4 layers of palisade tissue followed by compactly arranged spongy cells. Three or four layers of translucent palisade radially arranged above vascular bundles, furrows and entire area above midvein. Sclerenchyma fibers in abaxial corners of furrows. Vascular bundles: 35; commis- sural bundles not observed. One to three large vessels present in each bundle. Two phloem units lying laterally in flanges of V-shaped, some U-shaped, abaxial girder. Adaxial cap present on each bundle; usually quite large. Bundle sheath completely surrounding each bundle. Crystals and tannins: none observed.

TyPE.-Caraça, Minas Gerais, Brazil, cultivated and flowered in São Paulo, 10 January 1924, Hoehne $s n$ (SP 12495, holotype).

Distribution.-Known only from the typecollection.

\section{Vellozia ramosissima L. B. Smith}

Vellozia ramosissima L. B. Smith, Contr. U.S. Nat. Herb., 35:263, pl. 9: figs. 4, 5, 1962.

Type--Serra do Cipó $21 / 2$ miles $(4 \mathrm{~km})$ from Hotel Chapeu de Sol, Jaboticatubas, Minas Gerais, Brazil, $1110 \mathrm{~m}$ alt, 19 December 1959, Maguire b Pires 44666 (US, holotype; NY, isotype).

Distribution.-Known only from the typecollection.

\section{Vellozia bicarinata L. B. Smith \& Ayensu, new species}

Figure 13

A $V$. ramosissima L. B. Smith, cui parum affinis, scapo erecto, foliis flores plus minusve superantibus differt.

Caudex to $2 \mathrm{~m}$ high (!Maguire \& Pires), apparently much branched, ultimate branches $2 \mathrm{~cm}$ thick including leaf-sheaths. Leaves ca. 12-ranked; sheaths densely imbricate with minimum exposure, soon dull and fragmented; blades erect, quickly deciduous, linear, retuse, to $75 \mathrm{~mm}$ long, 7-8 mm wide at base, somewhat revolute when dry, subdensely vestite on both sides with minute sessile glands, the margins minutely serrulate.

Scape terminal, solitary, erect, mostly shorter than the leaves, laxly and minutely scabrous. Ovary obovoid, $15 \mathrm{~mm}$ long, laxly and minutely scabrous; epigynous tube broadly funnelform, $5 \mathrm{~mm}$ high. Tepals elliptic; broadly rounded and apiculate, 3 cm long, purple, glabrous. Stamens ca. 18; phalange appendages coarsely lacerate; anthers ca. $1 \mathrm{~cm}$ long. Style $3 \mathrm{~cm}$ long; stigmas deflexed.

Leaf Anatomy (Maguire \& Pires 44639).-Sur- 


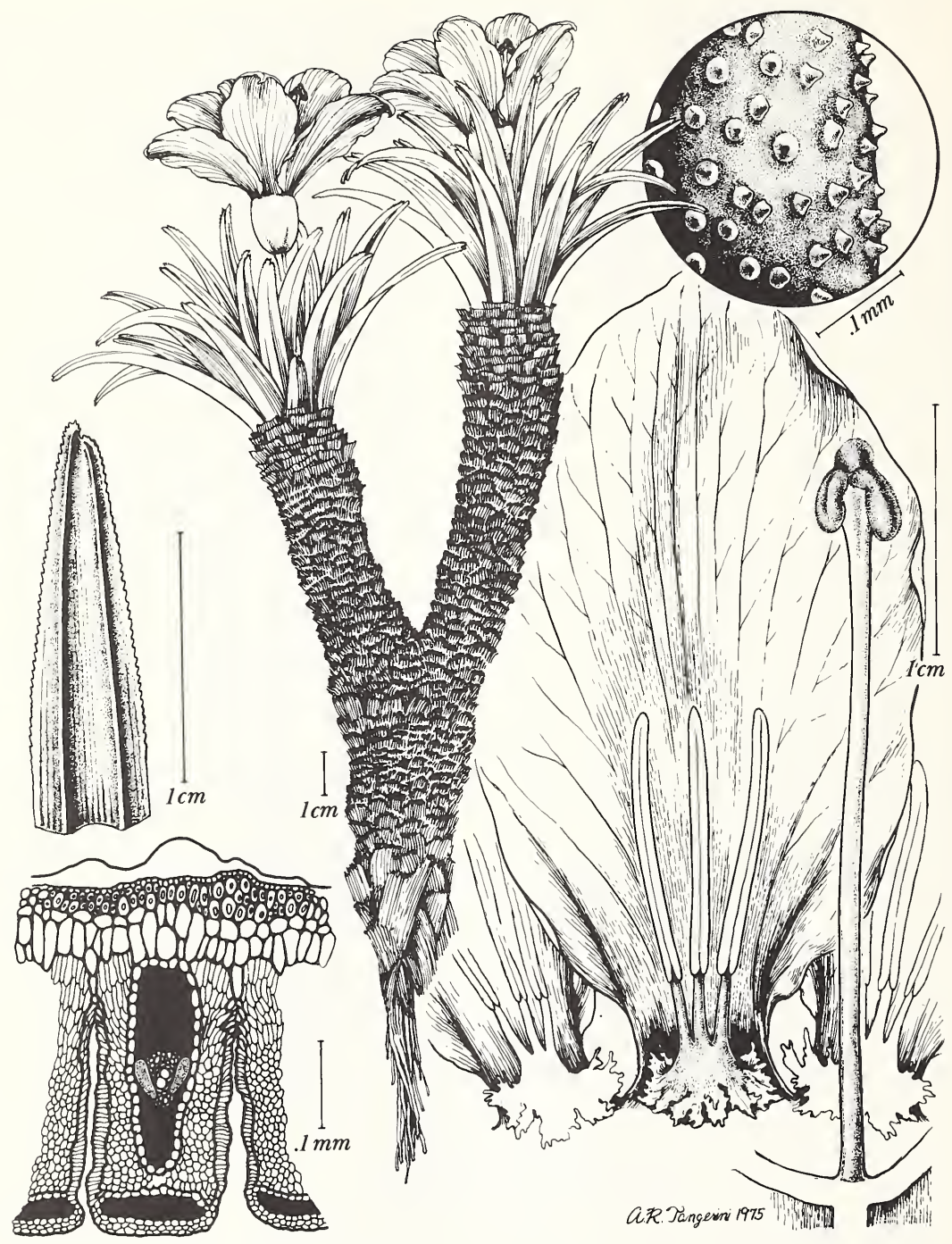

Figure 13.-Vellozia bicarinata L. B. Smith \& Ayensu, new species (Maguire \& Pires 44639). 
face View: Hairs: few tufts present on extreme margins. Epidermis: cells on both surfaces square to rectangular; walls slightly thickened. Stomata: paracytic and tetracytic, $18 \times 12 \mu \mathrm{m}$; present in abaxial furrows only.

Transverse Section of Lamina: Dorsiventral; widely V-shaped with small median adaxial groove. Adaxial surface ridged; abaxial surface furrowed about half to three-fourths thickness of blade. Epidermis: cells rounded to dome-shaped on both surfaces; walls slightly thickened. Subjacent to adaxial epidermis is a row of sclerenchyma fiber bundles interspersed with few thin-walled cells; below this is a layer of large, thin-walled cells. One or two layers of sclerenchyma fiber bundles subjacent to abaxial epidermis followed by a layer of rounded, thin-walled cells. Cuticle: very thick and ridged over entire surface; few conspicuous conical protrusions on adaxial cuticle. Stomata: present in abaxial furrows only; stomata flush with epidermal surface. Mesophyll: 2 or 3 layers of large, translucent palisade tissue followed by narrow, compactly arranged cells present on either side of each vascular bundle. This is followed by a compact spongy tissue. Vascular bundles: 35; commissural bundles few observed. One xylem vessel present in each bundle. Two phloem units lying laterally in flanges of short Y-shaped abaxial girder. Large adaxial cap present on each bundle. Bundle sheath completely surrounding each vascular bundle. Crystals: none observed. Tannins: few present.

Type.-Common on rocks, dominant, Serra de Cipó, $31 / 2$ miles $(5.6 \mathrm{~km})$ from Hotel Chapeu de Sol, Minas Gerais, Brazil, 3700 feet $(1110 \mathrm{~m})$ alt, 19 December 1959, Maguire of Pires 44639 (US, holotype; NY; RB, isotypes).

Distribution.-Brazil: Minas Gerais: Jaboticatubas: $6 \mathrm{~km}$ north of Palacio, October 1953, SegadasVianna \& Loredo Jr. no. Serra II-1085 (R, US).

\section{Subkey II}

34. Vellozia pterocarpa L. B. Smith \& Ayensu, new species

\section{FIGURE 14}

$\mathrm{Ab}$ omnibus speciebus adhuc cognitis ovario late tenuiterque alato, angulis glandulosis differt.

Caudex (incompletely known) over $12 \mathrm{~cm}$ high,
2-4 cm thick including old leaf-bases, simple(?). Leaves very densely imbricate; sheaths ca. $4 \mathrm{~cm}$ long; blades persistent, reflexed in age, lineartriangular, long-attenuate but minutely obtuse, to $19 \mathrm{~cm}$ long, $6 \mathrm{~mm}$ wide at base, laxly serrulate on the margins, otherwise even and glabrous.

Scapes 3, terminal, erect, 9-10 cm long, sulcate, very sparsely and minutely glandular. Ovary suborbicular, trigonous with broad thin glandularmargined wings on the angles, $14 \mathrm{~mm}$ long without the $7 \mathrm{~mm}$ long epigynous tube. Tepals lance-elliptic, $35 \mathrm{~mm}$ long, dark lilac (!Hatschbach), glabrous. Stamens about 24; phalange appendages papillate. Style exceeding the stamens; stigmas suborbicular, horizontal.

Leaf Anatomy (Hatschbach \& Ahumada 31705). -Surface View: Hairs: few, tufts of epidermal projections present on adaxial surface. Epidermis: cells square to rectangular on adaxial and abaxial surfaces. Stomata: paracytic, few tetracytic, $27 \times 15$ $\mu \mathrm{m}$; present in abaxial furrows.

Transverse Section of Lamina: Dorsiventral; almost horizontal with margins turned slightly downward. Adaxial surface ridged; abaxial surface furrowed 1/2 thickness of blade. Epidermis: cells on both surfaces small, rounded, and thick-walled. Cells fairly large and thin-walled above midvein. Subjacent to adaxial and abaxial epidermis is 1 or 2 layers of sclerenchyma cells followed by 1 layer of large, thin-walled parenchyma cells. Cuticle: slightly thickened and smooth over entire surface. Stomata: present in abaxial furrows only; flush with epidermal surface; small substomatal chamber present; stomata associated with projections from walls of furrows. Mesophyll: 4 layers of small, thin-walled palisade cells followed by 22 or 23 layers of equally small, closely packed, rounded cells. Two layers of large, translucent palisade cells above each bundle; large palisade cells plus 4 or 5 layers of small, spongy tissue abovie midvein. Vascular bundles: 25, commissural bundles not observed. One or two large vessels in each bundle. Two phloem units lying laterally in fairly long flanges of thin, Y-shaped abaxial girder. Small adaxial cap on each bundle. Bundles surrounded by distinct bundle sheath. Crystals: none observed. Tannins: few present.

TYPE.-Sandy campo, Guinda to Conceição da Mata, Diamantina, Minas Gerais, Brazil, 17 February 1973, Hatschbach \& Ahumada 31705 (US, holotype). 


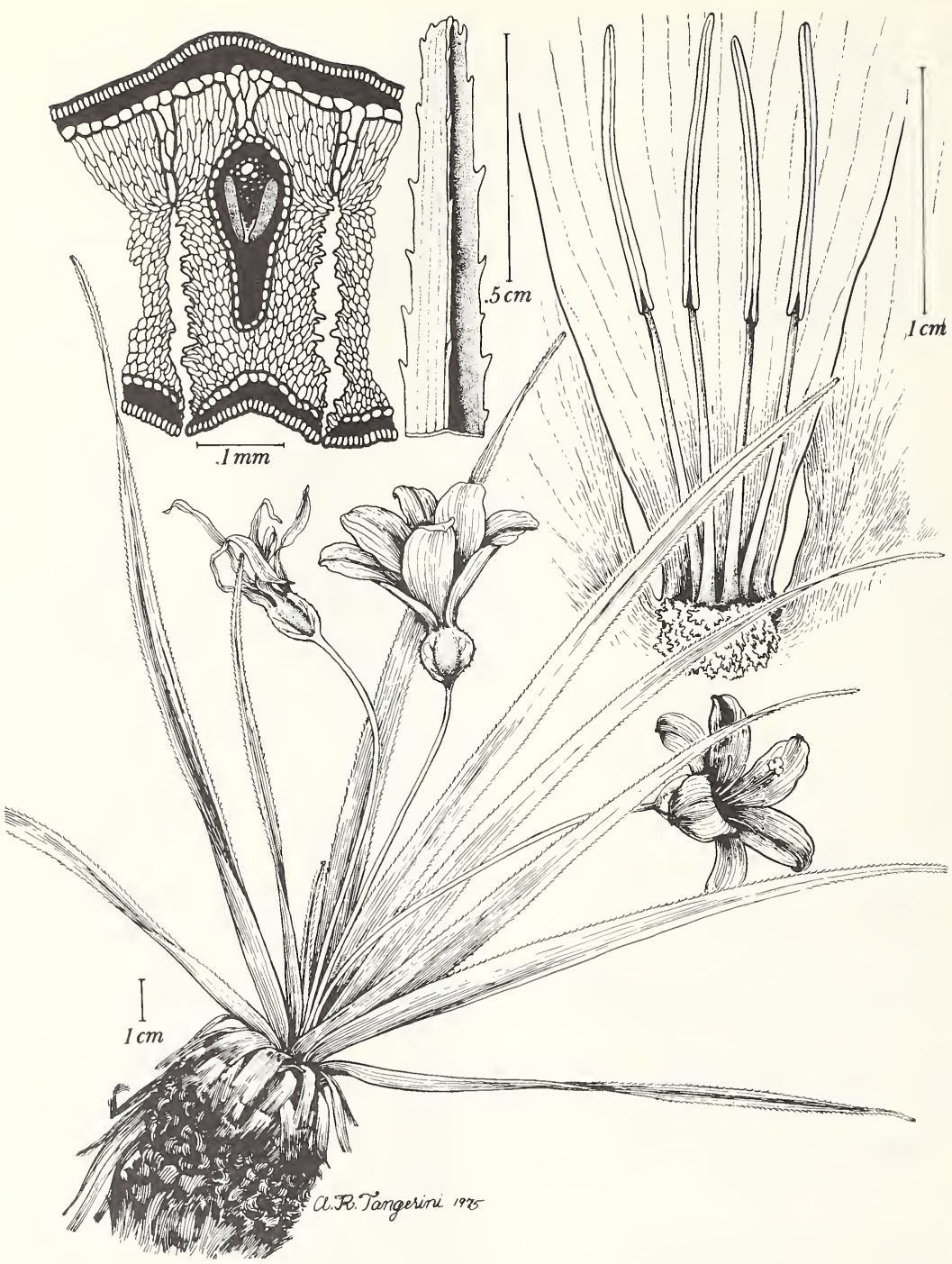

Figure 14.-Vellozia pterocarpa L. B. Smith \& Ayensu, new species (Hatschbach \& Ahumada 31705). 
Distribution.-Known only from the typecollection.

\section{Vellozia angustifolia Goethart \& Henrard}

Vellozia angustifolia Goethart \& Henrard, Blumea, 2:365, 1937.-L. B. Smith, Contr. U.S. Nat. Herb., 35:261, 1962.Ayensu, Smithsonian Contr. Bot., 15:12, fig. 7d-f, pl. 28a-e, 1974.

TyPE.-Among rocks, Morro Cubatão, near Guariroba, Goiás, Brazil, 11 April 1895, Glaziou 22213 (L, holotype (?); P, isotype; US, photo).

Distribution.-Brazil: Minas Gerais: Diamantina, Guinda. Goiás: Guariroba.

\section{Vellozia caruncularis Martius ex Seubert}

Vellozia caruncularis Martius ex Seubert in Martius, Fl. Bras., 3(1):78, pl. 8: fig. 1, 1847.-L. B. Smith, Contr. U.S. Nat. Herb., 35:267, 1962.-Ayensu, Smithsonian Contr. Bot., 15:15, fig. 14a-c, pls. 18b, 34b-d, 1974.

Vellozia droseroides L. B. Smith, Phytologia, 8:511, figs. 6, 7, 1963 [based on Riedel 1053 (K, holotype), Serra da Lapa (Cipó), Minas Gerais, Brazil, November 1824].

TyPE.-Minas Gerais, Brazil, Ackermann in Martius Exsicc. Herb. Flor. Brasil. 726 (M, holotype; $\mathrm{F}$, photo $18970 ; \mathrm{P}$, isotype).

Distribution.-Brazil: Minas Gerais: Contagem, Datas, Jaboticatubas, Sêrro.

\section{Vellozia variabilis Martius ex Schultes f.}

Vellozia variabilis Martius ex Schultes f. in Roemer \& Schultes, Syst., 7:293, 1826.-Seubert in Martius, Fl. Bras., 3(1):77, 1847.-L. B. Smith, Contr. U.S. Nat. Herb., 35:260, 1962.-Ayensu, Smithsonian Contr. Bot., 15:44, pls. 12a-b, $27 \mathrm{a}-\mathrm{f}, 1974$.

37a. Vellozia variabilis var. variabilis

?Vellozia alutacea Pohl, Pl. Bras., 1:130, 1827 [type: high rocky ground, Ouro Preto, Minas Gerais, Brazil, January 1821, Pohl s $n$ (W. holotype lost)].

?Vellozia crassirama Goethart \& Henrard, Blumea, 2:368, 1937 [type: Campo near Retiro, Rio Forto, Goiás, 12 February 1895, Glaziou 22210 (L)].

Vellozia wettsteinii Goethart \& Henrard Blumea, 2:383, 1937 [type: left bank of Rio Grande, Sacramento to Jaguara, São Paulo, Brazil, 1901, Wacket s $n$ in Exped. Wettstein d Schiffner (L holotype, US photo)].
Capsule tuberculate on the angles only to completely glabrous.

Type.-Without locality, Minas Gerais, Brazil, Martius s n (M, holotype; F, photo 18987).

Distribution.-Bolivia: Santa Cruz: Santiago de Chiquitos. Brazil: Minas Gerais: Cantoni, Datas, Diamantina, Gouveia, Grão Mogul, Serra do Itabirito, Veadeiros. Goiás: Cristalina, Goiás Velho, Luziania, Serra Dourada.

\section{7b. Vellozia variabilis var. tuberculata Seubert}

Vellozia variabilis var. tuberculata Seubert in Martius, Fl. Bras., 3(1):77, 1847.-L. B. Smith, Contr. U.S. Nat. Herb., $35: 260,1962$.

Capsules with a row of tubercules on each side as well as on the angles.

TyPE.-Without locality, Minas Gerais, Brazil, Martius s $n$ (M, $\mathrm{n}$ v).

Distribution.-Brazil: Minas Gerais: Caldas.

38. Vellozia resinosa Martius ex Schultes $f$.

FIGURE 15

Vellozia resinosa Martius, ex Schultes f. in Roemer \& Schultes, Syst., 7:293, 1826.-Seubert in Martius, F1. Bras., 3(1):80, 1847.-[emend.] L. B. Smith, Contr. U.S. Nat. Herb., 35:262, 1962.-Ayensu, Smithsonian Contr. Bot., 15:37, fig. 8a-c, pls. $30 \mathrm{~g}-\mathrm{h}, 31 \mathrm{a}, 1974$.

Vellozia irwinii L. B. Smith in Ayensu, Smithsonian Contr. Bot., 15:29, pl. 43a, 1974 [nomen.; based on Irwin, Maxwell ow Wasshausen 20998 (NY, US), rocky campo, $17 \mathrm{~km}$ north of Sêrro, Serra do Espinhaço, Minas Gerais, Brazil, $1200 \mathrm{~m}$ alt, 27 February 1968].

TyPE.-Without locality, Minas Gerais, Brazil, Martius s $n$ (M, holotype; F, photo 18982).

Distribution.-Brazil: Minas Gerais: Serra do Cipó, Sêrro.

39. Vellozia teres L. B. Smith \& Ayensu, new species

Figure 16

A V. variabile Martius ex Schultes f., cui affinis, tepalis minoribus, ovario haud trigono differt.

Caudex (imperfectly known) simple (?), $15 \mathrm{~cm}$ high (!Irwin), $1 \mathrm{~cm}$ thick including leaf-sheaths. Leaves at least 11 complete in the apical fascicle; sheaths densely imbricate (?), at least $3 \mathrm{~cm}$ long, 


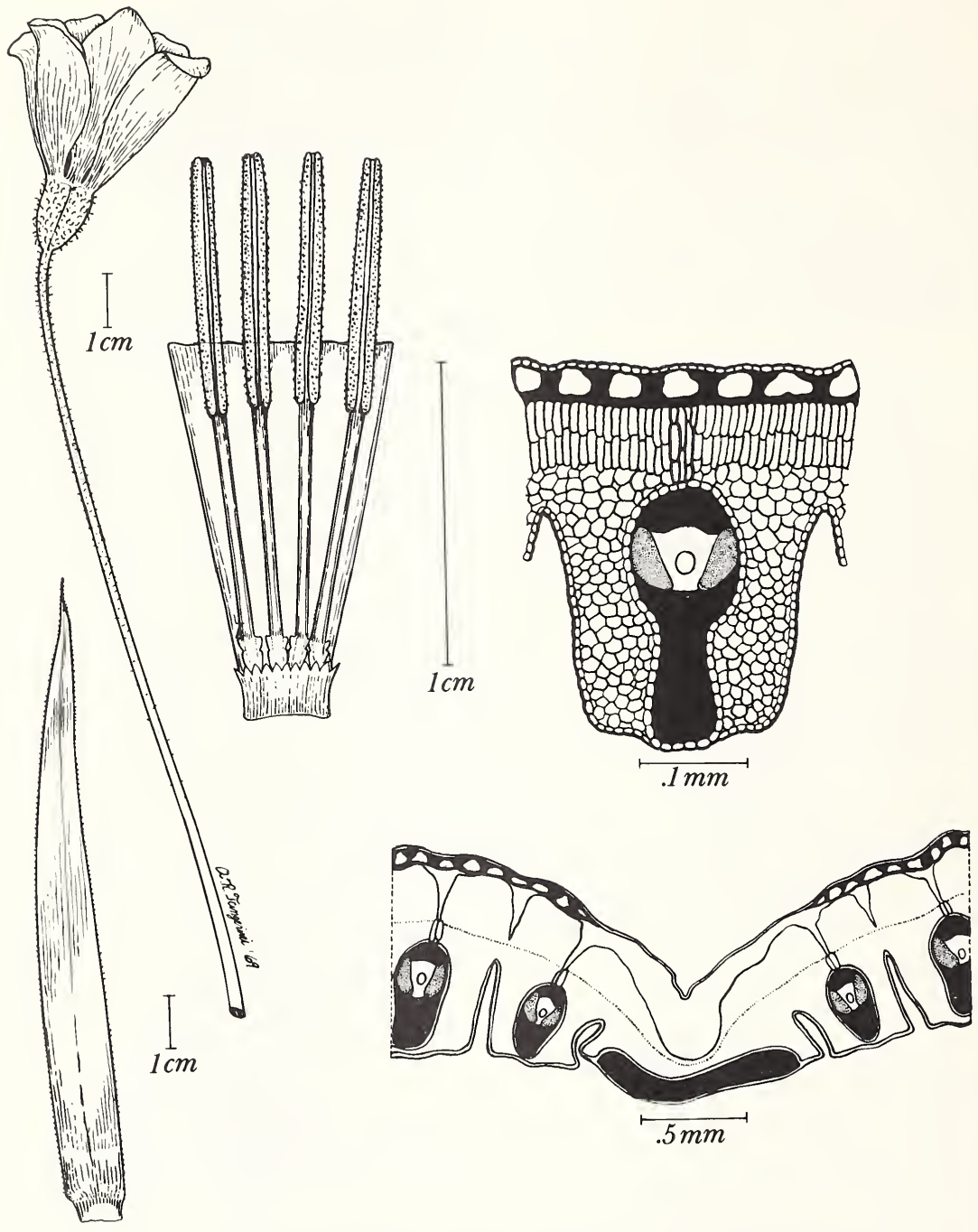

Figure 15.-Vellozia resinosa Martius ex Schultes f. (Irwin, Maxwell \& Wasshausen 20998). 


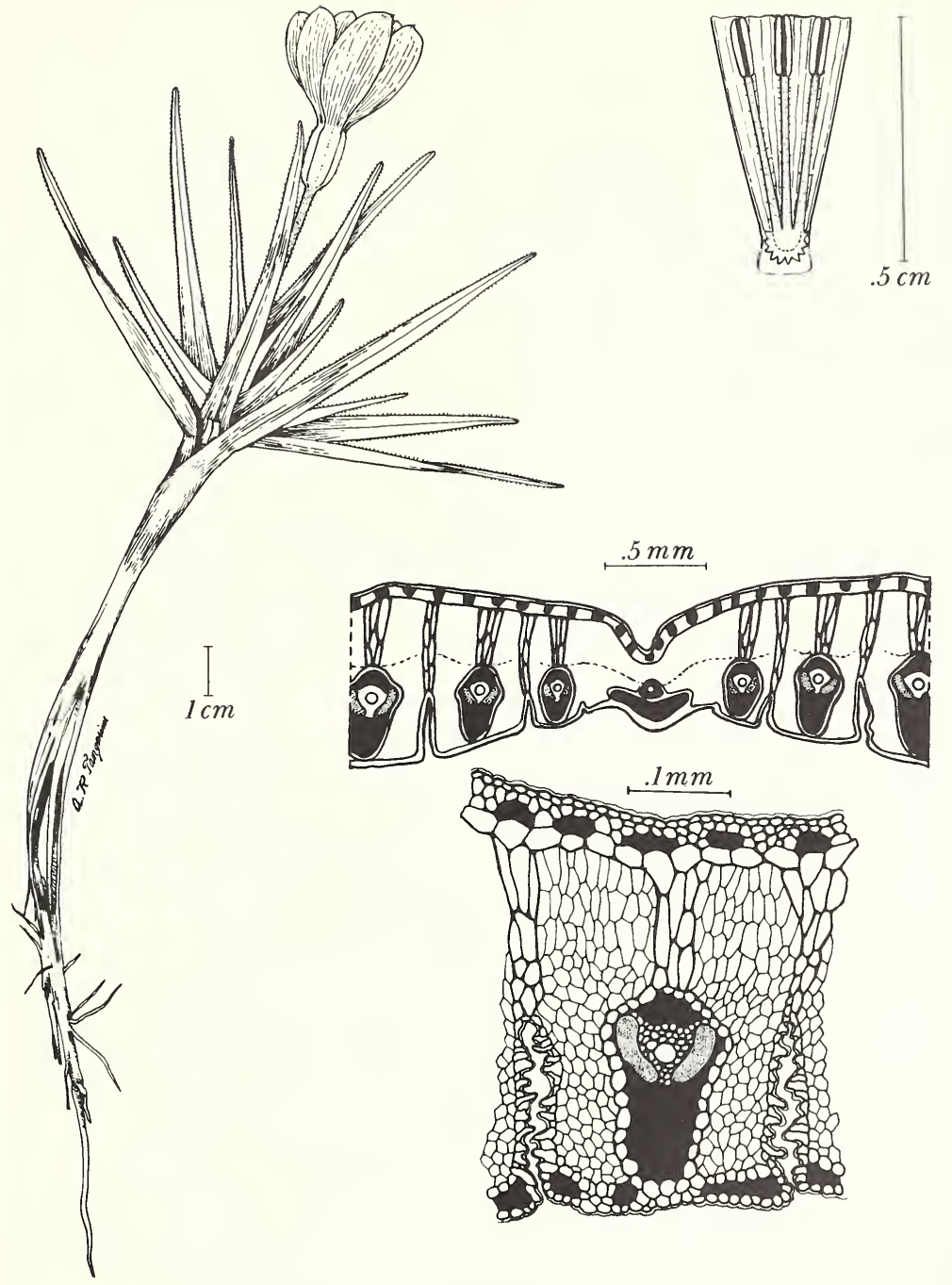

Figure 16.-Vellozia teres L. B. Smith \& Ayensu, new species (Irwin, Maxwell \& Wasshausen 21000). 
glutinous at apex, otherwise even and glabrous; blades very narrowly triangular, to $75 \mathrm{~mm}$ long, 6 $\mathrm{mm}$ wide at base, bicarinate above toward apex, densely and minutely serrulate on the margins and keels, otherwise even and glabrous.

Scape terminal, solitary, erect, shorter than the leaves, sulcate, minutely stipitate-glandular toward apex. Ovary ellipsoid, $13 \mathrm{~mm}$ long including the broad $2 \mathrm{~mm}$ long epigynous tube, sparsely sessileglandular on the centers of the carpels, otherwise even and lustrous. Tepals elliptic, broadly rounded and apiculate, $25 \mathrm{~mm}$ long, violet (!Irwin), glabrous. Stamens ca. 18-24, phalange-appendages coarsely dentate, anthers $9 \mathrm{~mm}$ long.

Leaf ANAтоmy.-Ayensu, Smithsonian Contr. Bot., 15:42, pl. 44e, 1974.

TYPE.--On outcrops, rocky campo, Serra do Espinhaço, ca. $17 \mathrm{~km}$ north of Sêrro on road (MG 2) to Diamantina, Minas Gerais, Brazil, $1200 \mathrm{~m}$ alt, 27 February 1968, Irwin, Maxwell \& Wasshausen 21000 (US, holotype; NY, isotype).

Distribution.-Known only from the typecollection. 40. Vellozia torquata L. B. Smith \& Ayensu,
new species

Figure 17

A $V$. variabile Martius ex Schultes f. et fere omnibus speciebus alteris scapis articulatis differt.

Caudex to ca. $1.5 \mathrm{~m}$ tall (!Irwin), branched. Leaves ca. 6-ranked, nonglutinous; sheaths subdense with considerable apical exposure; blades soon reflexed and long-persistent, linear-triangular, attenuate to a narrowly obtuse apex, to $13 \mathrm{~cm}$ long, $12 \mathrm{~mm}$ wide, faintly bicarinate above, nearly flat, laxly serrulate with suberect white spines, otherwise even and glabrous.

Scapes terminal, 3 (typically), $4 \mathrm{~cm}$ long (above the leaf-sheaths), articulate near apex, sulcate, laxly and minutely stipitate-glandular. Ovary ellipsoid, then contracted into a cylindrical epigynous tube, in all $12 \mathrm{~mm}$ long, subterete, laxly glandular in vertical lines. Tepals elliptic, $23 \mathrm{~mm}$ long. Stamens ca. 36, phalanges irregular, fimbriate-appendaged.

LeAF ANATomy (Irwin 27737).--Surface View: Hairs: none observed. Epidermis: cells on both surfaces square to rectangular; thin-walled. Stomata: tetracytic?, $21 \times 12 \mu \mathrm{m}$; present in abaxial furrows only.

Transverse Section of Lamina: Dorsiventral; widely V-shaped with margins turned slightly downward. Adaxial surface undulating; abaxial surface furrowed $1 / 2$ thickness of blade. Epidermis: adaxial and abaxial cells rounded to dome-shaped; few conical; thin-walled. Few abaxial cells replaced by sclerenchyma fiber bundles of 2 or 3 layers. This is followed by one layer of large, thin-walled parenchyma cells. Cuticle: slightly thickened and ridged on adaxial surface; thin and smooth on abaxial surface. Stomata: present in abaxial furrows only; flush with surface; fairly large substomatal chamber present. Stomata protected by projections from walls of furrows. Mesophyll: 3 or 4 layers of small palisade cells followed by compactly arranged spongy cells. Two or three layers of large, translucent cells radially arranged above vascular bundles and abaxial furrows. Vascular bundles: 43; commissural bundles not observed. Two or three large vessels present in each bundle. Two phloem units lying lateral to xylem in flanges of U- or Y-shaped abaxial girder. Small adaxial cap present. Bundle sheath completely surrounding each vascular bundle. Crystals: few in mesophyll. Tannins: few present.

TYPE.-Steep slopes with cut-over gallery forest and adjacent serrado with interspersed outcrops ca. $23 \mathrm{~km}$ east of Diamantina, Minas Gerais, Brazil, $900 \mathrm{~m}$ alt, 17 March 1970, Irwin et al. 27737 (US, holotype; NY, isotype).

Distribution.-Known only from the typecollection.

\section{Vellozia compacta Martius ex Schultes f.}

Vellozia compacta Martius ex Schultes f. in Roemer \& Schultes, Syst., 7:293, 1826.-Seubert in Martius, F1. Bras., 3(1):77, pl. 10: fig. 1, 1847.-L. B. Smith, Contr. U.S. Nat. Herb., 35:260, 1962.-Ayensu, Smithsonian Contr. Bot., 15:16, fig. 7a-c, pl. 25b-f, 1974.

Vellozia compacta var. obtusiflora Seubert in Martius, Fl. Bras., 3(1):77, 1847 [the typical variety; the illustration agrees with the description as to stamen-length but not as to tepal-form].

Vellozia compacta var. acutiflora Seubert in Martius, Fl. Bras., 3(1):77, 1847 [type: Minas Gerais, Brazil, Sellow $s \quad n$ (herb. ?)].

Vellozia ambigua Goethart \& Henrard, Blumea, 2:364, 1937 [type: without locality, Brazil, Sellow s $n$ (B)].

Vellozia martiana Goethart \& Henrard, Blumea, 2:376, 1937 


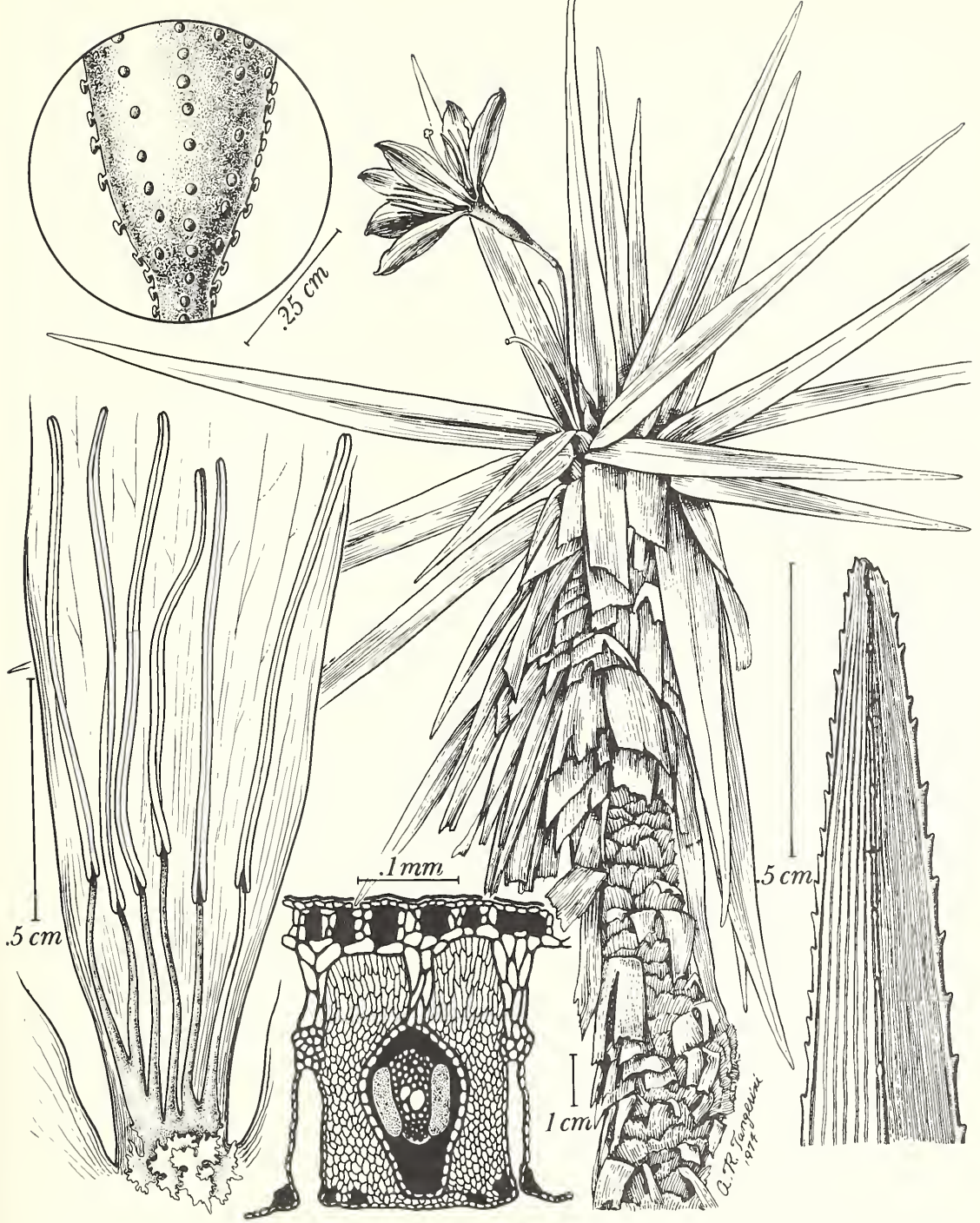

Figure 17.-Vellozia torquata L. B. Smith \& Ayensu, new species (Irwin 27737). 

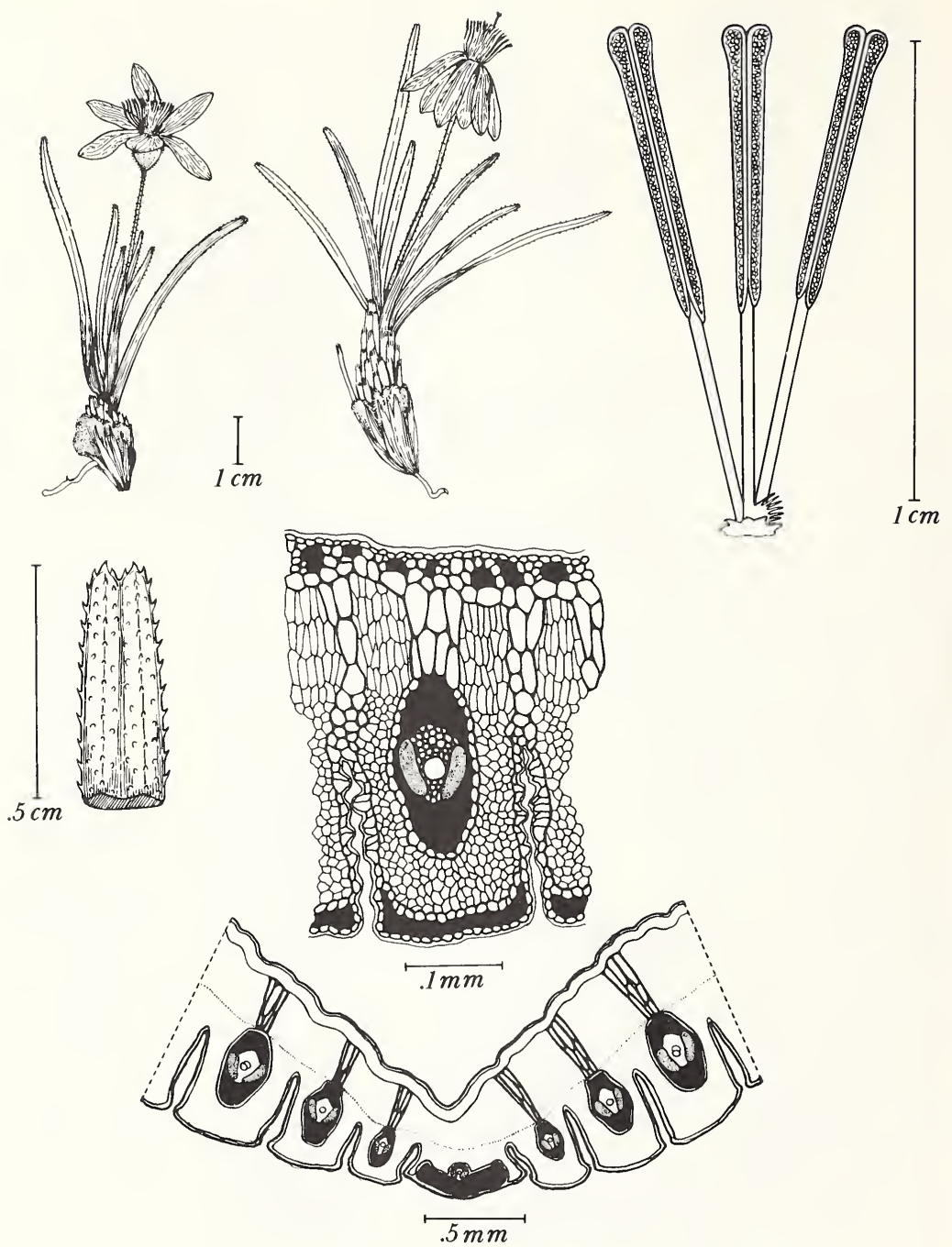

Figure 18.-Vellozia wasshausenii L. B. Smith \& Ayensu, new species (Irwin, Maxwell \& Wasshausen 20318). 
[type: without locality, Brazil, Martius, Herb. Fl. Bras. 725 (P, US photo). Possibly the same collection as the type of V. compacta].

Type-Without locality, Brazil, Martius $s$ n (herb.?).

Distribution.-Brazil: Minas Gerais: Datas, Pico de Itabira, Nova Lima, Serra da Piedade, São João da Chapada.

\section{Vellozia wasshausenii L. B. Smith \& Ayensu, new species}

\section{FIGURE 18}

A V. compacta Martius ex Schultes f., cui affinis, caudice brevi simplici, foliis floribusque minoribus, tepalis per anthesin reflexis differt.

Caudex simple, not more than $4 \mathrm{~cm}$ long and 1 $\mathrm{cm}$ thick including the old leaf-bases. Leaves fewranked with 5-8 in the apical fascicle; sheaths subdensely imbricate with up to $5 \mathrm{~mm}$ of apex exposed, glabrous, more or less lustrous at first, not splitting much with age; blades erect, soon deciduous, linear, retuse, $6 \mathrm{~cm}$ long, $3 \mathrm{~mm}$ wide at base, bicarinate above with the midnerve depressed, revolute, serrulate on margins, keels, and apex, otherwise even and glabrous.

Scape terminal, solitary, to $7 \mathrm{~cm}$ long, very slender, laxly stipitate-glandular. Ovary subglobose, $8 \mathrm{~mm}$ long, dark, laxly but rather evenly darkglandular. Tepals elliptic, obtuse, 15-20 mm long, light purple (!Irwin), free. Stamens 18, to $12 \mathrm{~mm}$ long; filaments dark purple; phalange-appendages mostly broad, crisped, undulate. Style exceeding the stamens; stigmas suborbicular, decurved.

Leaf Anatomy.-Ayensu, Smithsonian Contr. Bot., 15:45, pl. 45a, 1974.

TyPE.-On rock outcrop, Serra do Cipó, km 132 (about $153 \mathrm{~km}$ north of Belo Horizonte), Minas Gerais, Brazil, $1400 \mathrm{~m}, 16$ February 1963, Irwin, Maxwell \& Wasshausen 20318 (US, holotype; NY, isotype).

Distribution.-Known only from the typecollection.

\section{Vellozia fruticosa L. B. Smith}

Vellozia fruticosa L. B. Smith, Contr. U.S. Nat. Herb., 35:286, pl. 11: figs. $38,39,1962$.
TyPE.-On sandstone outcrop, ca, 3-5 km east of Sêrro, along road to Diamantina, Minas Gerais, Brazil, 9 August 1960, Maguire, Magalhães of Maguire 49130 (US, holotype; NY, isotype).

Distribution.-Known only from the typecollection.

\section{Vellozia patens L. B. Smith \& Ayensu, new species}

Figure 19

A $V$. caruncularis Martius ex Seubert, cui affinis, ovarii glandulis sessilibus vel subsessilibus differt.

Caudex few-branched, to $15 \mathrm{~cm}$ long, ca. $8 \mathrm{~mm}$ in diameter (including leaf-sheaths), forming dense clumps (!Irwin). Leaves few-ranked, glutinous; sheaths subdensely imbricate with ca. $4 \mathrm{~mm}$ exposed; blades soon reflexed, long-persistent, linear, attenuate to a narrowly obtuse apex, to $55 \mathrm{~mm}$ long, $4.5 \mathrm{~mm}$ wide, flat, laxly serrulate on margins and midnerve, otherwise even and glabrous.

Scapes solitary, erect or slightly curved, 15-30 $\mathrm{mm}$ long (above the leaf-sheaths), $1 \mathrm{~mm}$ in diameter, laxly glandular. Ovary ellipsoid, strongly trigonous, $15 \mathrm{~mm}$ high, bearing sessile or subsessile glands on the angles, the epigynous tube very short. Tepals withered, imperfectly known. Stamens ca. 18, ca. 6 mm long, phalanges unappendaged.

Leaf Anatomy (Irwin, Maxwell \& Wasshausen 20017).-Surface View: Hairs: few large tufts present on adaxial surface. Epidermis: cells square to rectangular on adaxial surface; rectangular on abaxial surface; thin-walled on both surfaces. Stomata: tetracytic, some paracytic, $21 \times 12 \mu \mathrm{m}$; present in rows along abaxial furrows.

Transverse Section of Lamina: Dorsiventral; widely $V$-shaped with small median adaxial groove and margins turned slightly downward. Adaxial surface ridged; abaxial furrowed $1 / 2$ thickness of blade. Epidermis: cells rounded to dome-shaped on both surfaces; few conical on abaxial surface, thinwalled. Subjacent to adaxial epidermis is 2 or 3 layers of thin-walled cells interspersed with rows of sclerenchyma fibers. This is followed by a layer of large, thin-walled parenchyma cells. No sclerenchyma above midvein. Cuticle: slightly thickened and smooth on adaxial surface; thin and smooth on abaxial surface. Stomata: present in abaxial furrows only; stomata above surface and associated 


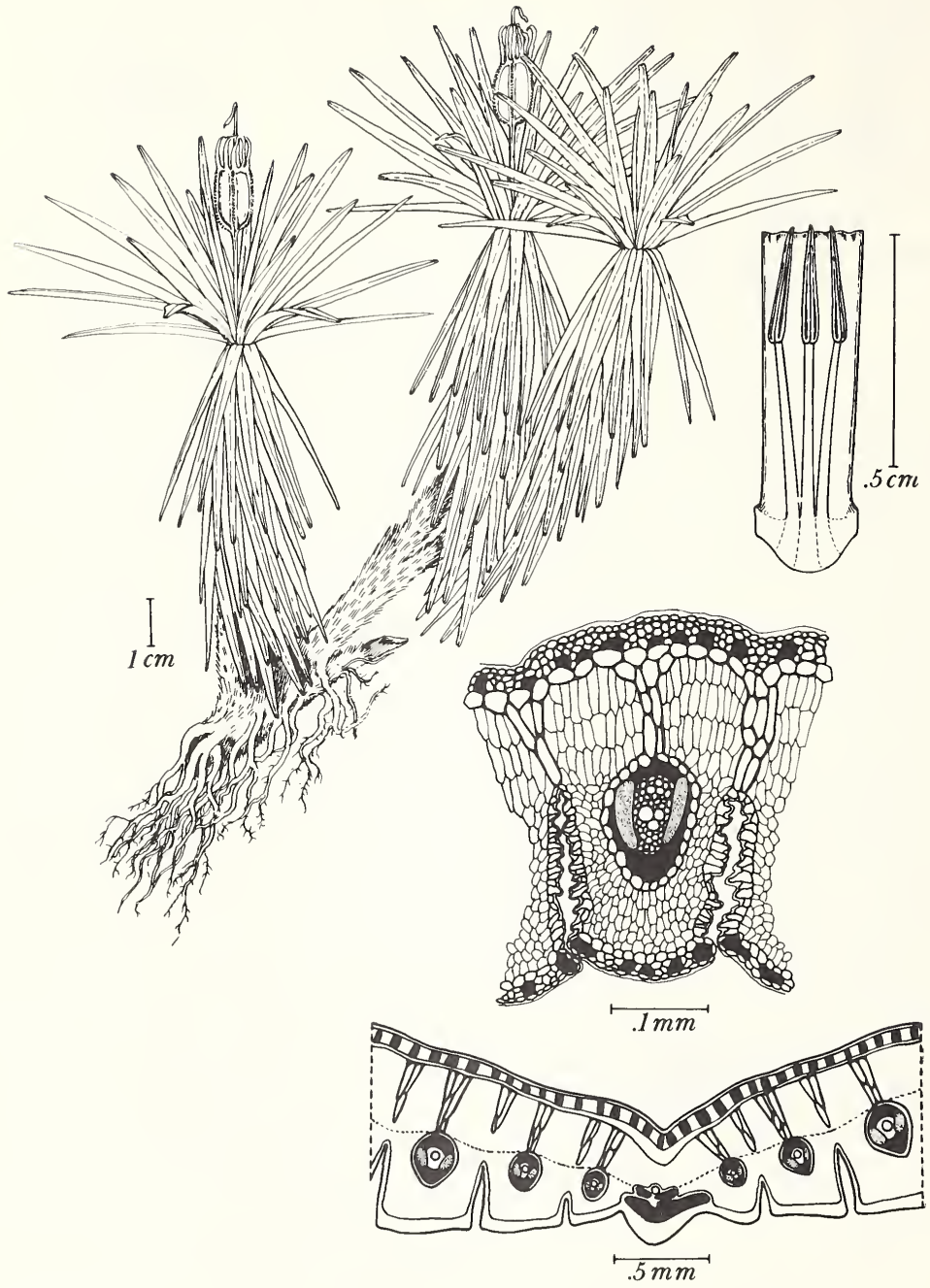

Figure 19.-Vellozia patens L. B. Smith \& Ayensu, new species (Irwin, Maxwell \& Wasshausen 20017). 
with projections along walls of furrows. Substomatal chamber present. Mesophyll: 3 layers of palisade tissue followed by closely packed spongy cells. Two or three layers of large, translucent palisade cells radially arranged above vascular bundles, furrows, and midvein. Vascular bundles: 24; commissural bundles not observed. One or two large vessels present in each bundle. Two phloem units lying laterally in flanges of $U$-shaped abaxial girder. Small adaxial cap present on each bundle. Sclerenchyma fibers present at abaxial corners of furrows and few interspersed with, and subjacent to, abaxial epidermis. Vascular bundles surrounded by bundle sheath. Crystals: none observed. Tannins: few present.

TYPE.-On rock outcrops in sandy campo, Serra do Cipó, ca. km 120 (ca. $145 \mathrm{~km}$ north of Belo Horizonte), Minas Gerais, Brazil, $1200 \mathrm{~m}$ alt, 14 February 1968, Irwin, Maxwell \& Wasshausen 20017 (US, holotype; NY, isotype).

Distribution.-Brazil: Minas Gerais: km 111112, road from Chapeu do Sol to Conceição do Mato Dentro, 4 July 1969, Menezes of Sazima 52 (SPF, US); km 138, 8 September 1972, Menezes 261 (SPF, US).

\section{Vellozia modesta L. B. Smith \& Ayensu, new species}

Figure 20

A $V$. carunculare Martius ex Seubert, cui affinis, foliis latioribus, ovario terete, glandulis subsessilibus differt.

Caudex, few-branched, $1.5 \mathrm{~m}$ high (including leaf-sheaths), 15 (-25) $\mathrm{mm}$ thick at apex. Leaves ca. 7 in the terminal erect fascicle; sheaths densely nerved, remaining entire; blades reflexing and persistent, linear obtuse or subacute, $12(-18) \mathrm{cm}$ long and $12 \mathrm{~mm}$ wide, dry, even and glabrous except for keels and margins, bicarinate and serrulate above toward apex, the margins at least in part densely serrulate with inconspicuous slender biserriate teeth.

Scapes solitary, erect, $3(-7) \mathrm{cm}$ long (above the leaf-sheaths), ca. $1 \mathrm{~mm}$ in diameter, sulcate, subdensely vestite with subsessile glands. Ovary slenderly obovoid, terete, $7(-9) \mathrm{mm}$ long, laxly to densely covered with dark subsessile glands. Tepals free, elliptic, apiculate, $2 \mathrm{~cm}$ long, more or less glandular on base and midnerve. Stamens ca. 30 $(-40)$, the phalanges lacerate-appendaged.

Leaf Anatomy.-Ayensu, Smithsonian Contr. Bot., 15:33, pl. 43d, 1974.

TYPE.-On rock outcrop, $20 \mathrm{~km}$ north of Diamantina, Mun. Diamantina, Minas Gerais, Brazil, alt. 1300 m, 20 January 1972, Smith, Ayensu b Hatschbach (separate no. 29001) 15997 (holotype US, isotype MBM).

Distribution.-Brazil: Minas Gerais: moist sandy soil, ca. km 115, Serra do Cipó, 9 March 1969, Joly \& Menezes 20 (SPF).

Note: The paratype specimen differs from the type in certain measurements which have been added to the description in parentheses. Also its leaves are more prominently serrulate than those of the type.

\section{Vellozia breviscapa Martius ex Schultes f.}

Figure 21

Vellozia breviscapa Martius ex Schultes f. in Roemer \& Schultes, Syst., 7:293, 1826.-Seubert in Martius, Fl. Bras., 3(1):79, 1847.-L. B. Smith, Contr. U.S. Nat. Herb., 35:261, 1962.

Vellozia lappa L. B. Smith \& Ayensu in Ayensu, Smithsonian Contr. Bot., 15:30, p1. 37d, 1974 [nomen.; based on L. B. Smith, Ayensu \& Hatschbach 15988 (US), Guinda, Diamantina, Minas Gerais, Brazil, 19 January 1972].

Type-Without locality, Minas Gerais, Brazil, Martius s $n$ (M, holotype; F, photo 18969).

Distribution.-Brazil: Minas Gerais: Diamantina.

\section{Vellozia fimbriata Goethart \& Henrard}

Vellozia fimbriata Goethart \& Henrard, Blumea, 2:371, 1937. L. B. Smith, Contr. U.S. Nat. Herb., 35:261, 1962.

Type.-Serra da Lapa (Cipó), Minas Gerais, Brazil, Langsdorff s $n$ (LE, holotype; n. v.).

Distribution.-Known only from the type-collection.

\section{Vellozia barbaceniifolia Seubert}

Figure 22

Vellozia barbaceniifolia Seubert in Martius, Fl. Bras., 3(1):79, 1847.-L. B. Smith, Contr. U.S. Nat. Herb., 35:261, 1962. 


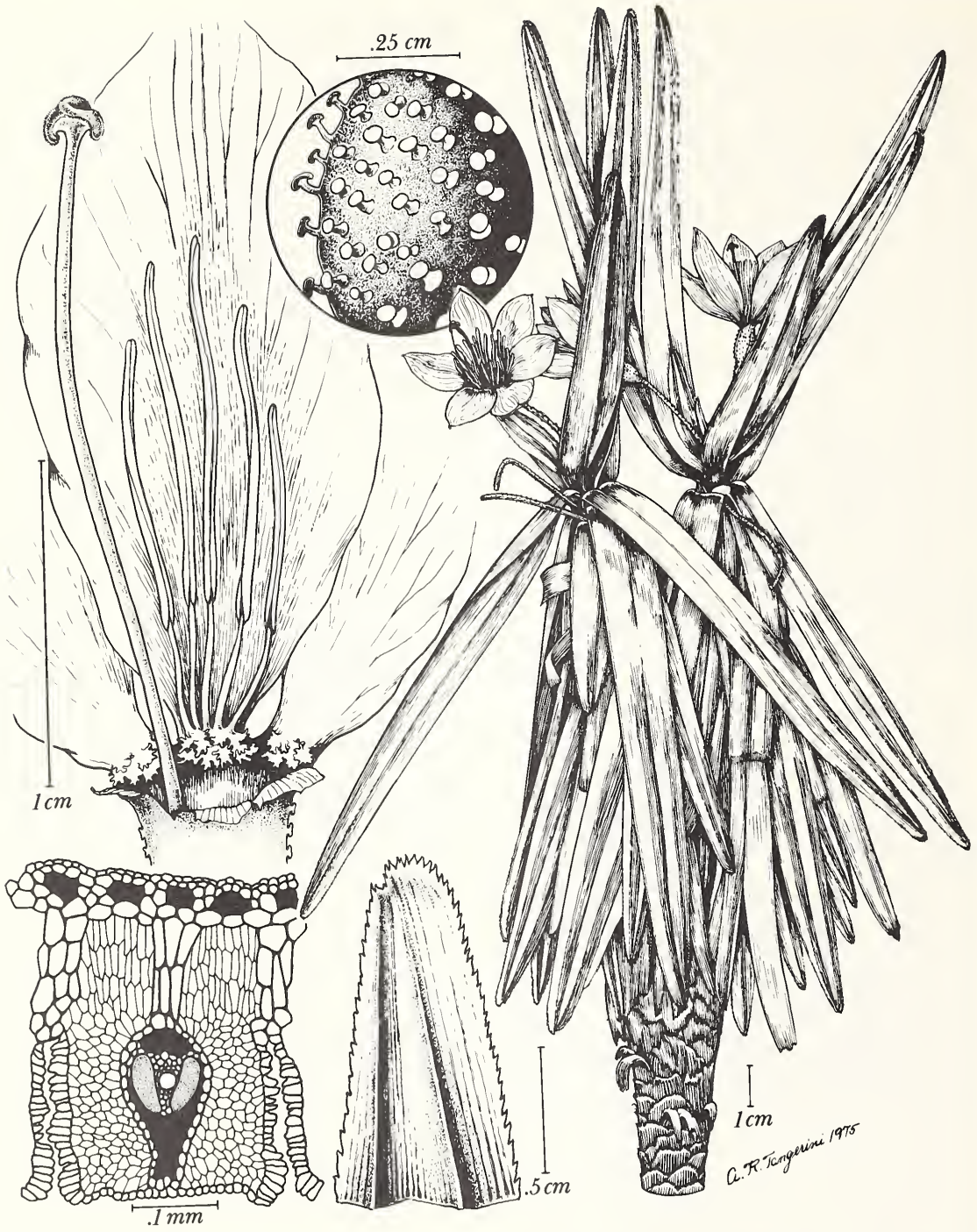

Figure 20.-Vellozia modesta L. B. Smith \& Ayensu, new species (L. B. Smith, Ayensu \& Hatschbach 15997). 


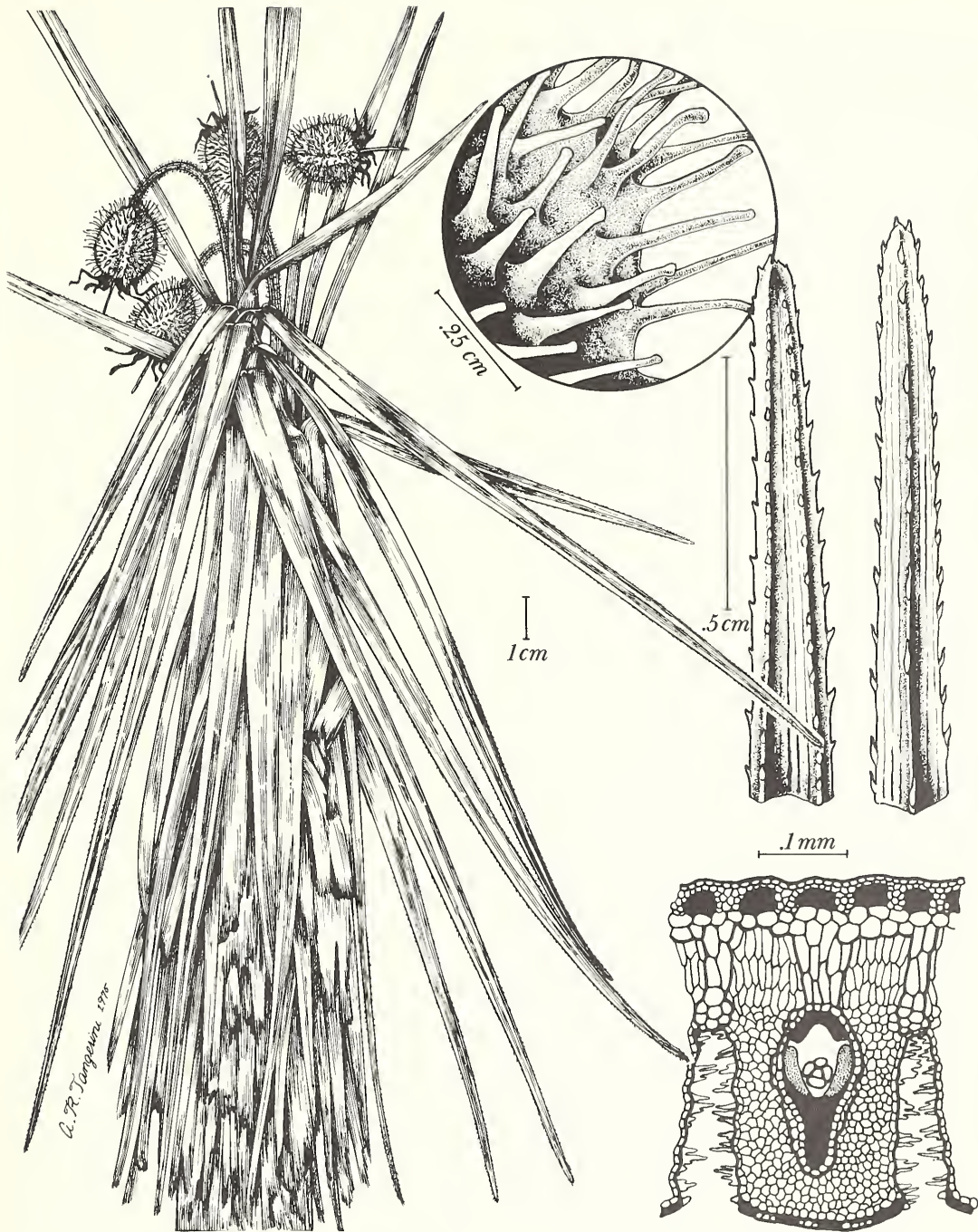

Figure 21.-Vellozia breviscapa Martius ex Schultes f. (L. B. Smith, Ayensu \& Hatschbach 15988). 


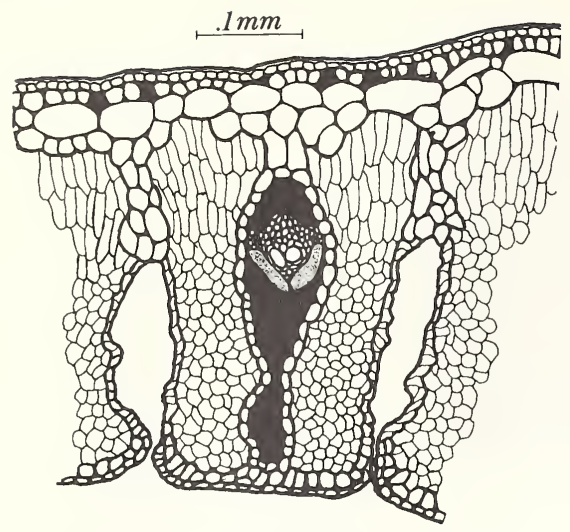

Figure 22.-Vellozia barbaceniifolia Seubert (Sellow $s$ ).

LeAF ANATomy (Sellow $s \quad n)$.-Surface View: Hairs: few tufts observed on adaxial surface. Epidermis: cells on both surfaces square to rectangular; thin-walled. Stomata: tetracytic, $15 \times 12 \mu \mathrm{m}$; most present in abaxial furrows; few on abaxial surface.

Transverse Section of Lamina: Dorsiventral; V-shaped with small median adaxial groove and margins curved down. Adaxial surface slightly undulating; abaxial furrowed $1 / 2$ thickness of blade. Epidermis: adaxial and abaxial cells rounded to dome-shaped; outer tangential wall slightly thickened; sclerenchyma fibers replacing some epidermal cells on both surfaces. Subjacent to adaxial epidermis is a layer of slightly thickened cells interspersed with sclerenchyma fibers; below is a layer of large parenchyma cells. Subjacent to abaxial epidermis is a layer of large parenchyma cells. Cuticle: thin and smooth on both surfaces. Stomata: mostly present in abaxial furrows; few on abaxial surface; stomata flush with surface; small projections observed in furrowed walls; small substomatal chamber present. Mesophyll: 3 or 4 layers of palisade cells followed by compactly arranged spongy mesophyll; 3 or 4 layers of translucent palisade cells radially arranged above vascular bundles, furrows, and midvein. Vascular bundles: 30; commissural bundles observed. One or two large vessels present in each bundle, mostly one.
Two phloem units lying laterally in flanges of thin abaxial girder. Adaxial cap present on each bundle. Bundle sheath completely surrounding each bundle. Crystals and tannins: few present.

TyPE.-Without locality, Brazil, Sellow $s \quad n$ (B, holotype; US, photo).

Distribution.-Known only from the typecollection.

\section{Vellozia viannae L. B. Smith}

Vellozia viannae L. B. Smith, Contr. U.S. Nat. Herb., 35:261, pl. 9: fig. 1, 1962.

TYPE.-Some $6 \mathrm{~km}$ north of Palacio, Serra do Cipó, Jaboticatubas, Minas Gerais, Brazil, October 1953, Segadas-Vianna do Lorêdo Jr. no. Serra-II, 1103 (US, holotype; R, isotype).

Distribution.-Known only from the typecollection.

\section{Vellozia subalata L. B. Smith \& Ayensu, new species}

\section{Figure 23}

A $V$. viannae L. B. Smith, cui parum affinis, capsula subduplo majore, carpellis subalatis differt.

Caudex ("like that of $V$. alata") tall and stout, but branched. Leaves (detached) with blades sharply curved at base and so probably reflexed and persistent, very narrowly triangular, caudate-attenuate, over $7 \mathrm{dm}$ long, $3 \mathrm{~cm}$ wide at base, flat, dull when dry, margins pectinate-serrate with divergent slender spines $4 \mathrm{~mm}$ long, elsewhere even and glabrous.

Scapes curved when dry, 13-15 cm long in all, sulcate, laxly and finely glandular. Ovary subglobose, obtusely trigonous, $27 \mathrm{~mm}$ long including the epigynous tube, laxly and finely stipitateglandular. Tepals withered, unknown. Stamens largely unknown but apparently at least 3 per tepal; phalange tall and narrow, unappendaged.

Leaf Anatomy (Menezes 193).-Surface View: Hairs: tufts present on both surfaces. Epidermis: cells on both surfaces square to rectangular; thinwalled. Stomata: tetracytic, $21 \times 12 \mu \mathrm{m}$; mostly in abaxial furrows; few on both surfaces.

Transverse Section of Lamina: Dorsiventral; 


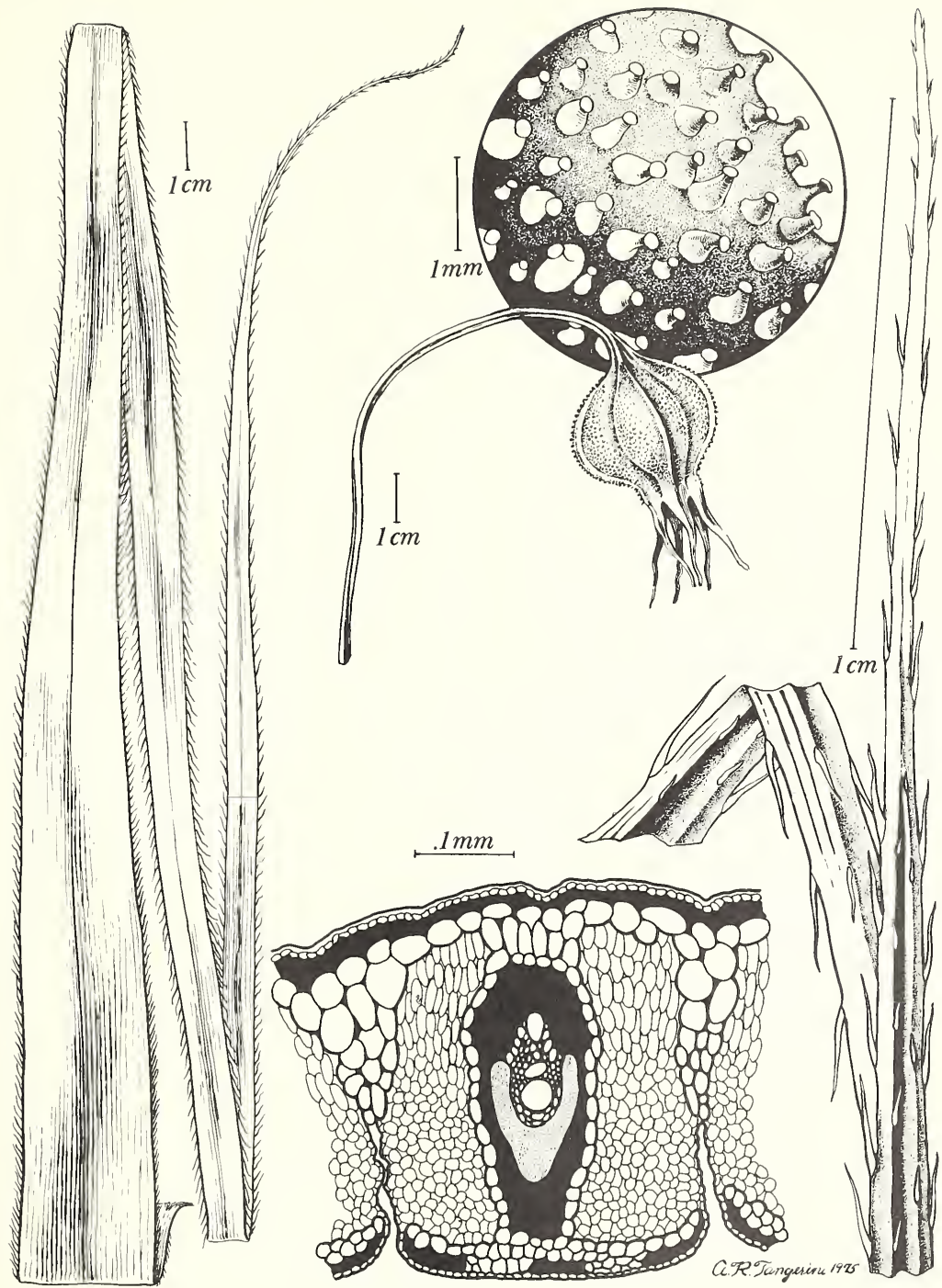

Figure 23.-Vellozia subalata L. B. Smith \& Ayensu, new species (Menezes 193). 
widely V-shaped. Adaxial surface slightly ridged; abaxial surface furrowed $1 / 3$ thickness of blade. Epidermis: adaxial cells rounded to dome-shaped; few conical, especially in area above midvein; thinwalled. Subjacent to adaxial epidermis is a layer of small, rounded, thin-walled cells interspersed with sclerenchyma fibers; below this is a single layer of large, thin-walled parenchyma cells. Abaxial epidermis consists of small, rounded, thin-walled cells, most of which have been replaced by sclerenchyma fibers. Subjacent is 1 or 2 layers of thin-walled parenchyma cells. Cuticle: thickened and slightly ridged over entire surface. Stomata: present in abaxial furrows only; stomata flush with surface and with a small substomatal chamber, accompanied by few projections from walls of furrows. Mesophyll: 3 or 4 layers of small, palisade tissue followed by compactly arranged spongy tissue. Two or three layers of large, translucent palisade cells radially arranged above vascular bundles and furrows. Many translucent cells above midvein. Vascular bundles: 19; commissural bundles observed. One or two large vessels present in each bundle, mainly one. Two phloem units lying laterally in flanges of short, Y-shaped abaxial girder. Adaxial cap present on each bundle. Vascular bundles surrounded with bundle sheath. Crystals: none observed. Tannins: few present.

Type- - Km 10-18 of the road between Chapeu do Sol and the dam on the Rio Cipó, Minas Gerais, Brazil, 11 December 1971, Menezes 193 (SP, holotype; US, photo).

Distribution.-Known only from the typecollection.

\section{Vellozia intermedia Seubert}

Figure 24

Vellozia intermedia Seubert in Martius, Fl. Bras., 3(1):78, 1847.-L. B. Smith, Contr. U.S. Nat. Herb., 35:261, 1962.Ayensu, Smithsonian Contr. Bot., 15:29, pl. 28f-g, 1974.

Trpe.-Without locality, Brazil, Sellow s $n$ (B, holotype; US, photo).

Distribution.-Cunha Magalhães, north of Diamantina, Minas Gerais, Brazil.

\section{Vellozia tomeana L. B. Smith \& Ayensu, new species}

Figure 25

A $V$. intermedia Seubert, cui affinis, ovario ellipsoideo haud angulato differt.

Caudex stout (only extreme apex known). Leaves many-ranked, glutinous; sheaths apparently very dense with little or no apical exposure, $45 \mathrm{~mm}$ long, yellow, even and glabrous; blades reflexing and presumably persistent, very narrowly triangular, finely attenuate, $35 \mathrm{~cm}$ long, $12 \mathrm{~mm}$ wide, flat, erect-serrulate, otherwise even and glabrous.

Scapes solitary, slightly curved, $10 \mathrm{~cm}$ long (above the leaf-sheaths), over $2 \mathrm{~mm}$ in diameter, scarcely sulcate, laxly and finely stipitate-glandular. Ovary ellipsoid, terete, $12 \mathrm{~mm}$ long, laxly and evenly vestite with fine black subsessile glands; epigynous tube very short. Tepals elliptic, $35 \mathrm{~mm}$ long, blue (!Hatschbach). Stamens ca. 50; phalanges with small entire appendages.

LeAf ANATOMy (Hatschbach \& Ahumada 31259). -Surface View: Hairs: few large tufts present on both surfaces. Epidermis: cells square to rectangular on both surfaces; thin-walled. Stomata: tetracytic, $21 \times 12 \mu \mathrm{m}$; present in abaxial furrows only.

Transverse Section of Lamina: Dorsiventral; very widely V-shaped with extreme margins turned slightly downward. Adaxial surface slightly ridged; abaxial surface furrowed $1 / 2$ to $3 / 4$ thickness of blade. Epidermis: adaxial cells rounded to domeshaped; few conical, especially above midvein; thinwalled; interspersed with sclerenchyma fibers. Subjacent to adaxial epidermis is 1 or 2 layers of sclerenchyma fiber bundles interspersed with thinwalled cells. This is followed by a layer of fairly large, thin-walled parenchyma cells. Abaxial cells rounded to dome-shaped; few conical; thin-walled. Cuticle: slightly thickened and ridged on adaxial and abaxial surfaces. Stomata: present in abaxial furrows only; stomata raised above surface; small substomatal chamber present. Stomata associated with projections from walls of furrows. Mesophyll: 2 or 3 layers of palisade tissue followed by compactly arranged spongy cells. Two or three layers of large translucent cells radially arranged above vascular bundles, abaxial furrows and midvein. Vascular bundles: 35; commissural bundles not observed. One or two large vessels in each vascular 


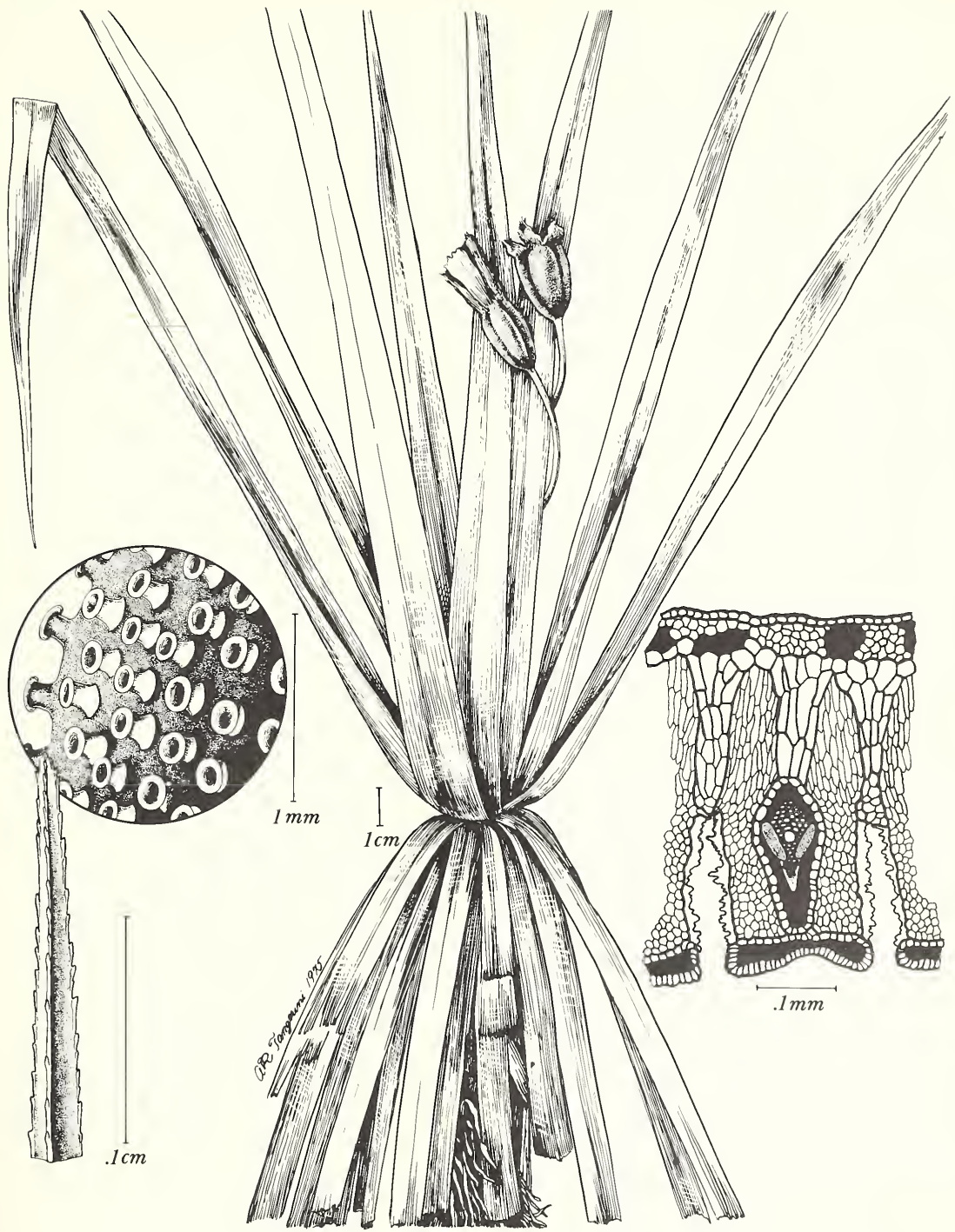

Figure 24.-Vellozia intermedia Seubert (Sellow $s n$ ). 


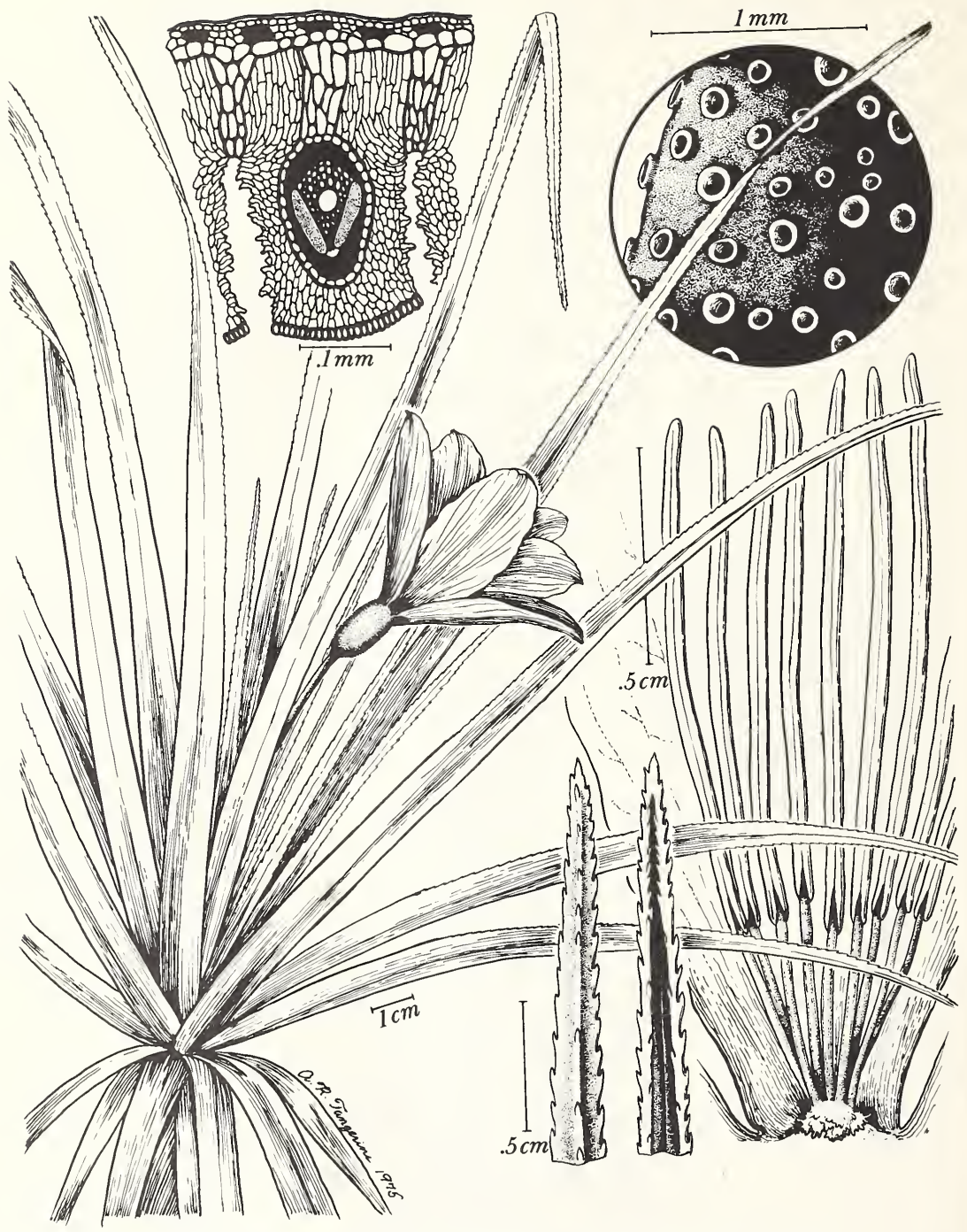

Figure 25.-Vellozia tomeana L. B. Smith \& Ayensu, new species (Hatschbach \& Ahumada 31259). 
bundle, mostly one. Two phloem units lying laterally in flanges of $\mathrm{Y}$-shaped abaxial girder. Adaxial cap present on each bundle. Bundle sheath completely surrounding each vascular bundle. Crystals: none observed. Tannins: few present.

TYPE.-Rock outcrops on hillside, São Tomé das Letras, Minas Gerais, Brazil, 5 February 1973, Hatschbach of Ahumada 31259 (US, holotype; MBM, isotype).

\section{Vellozia grao-mogulensis L. B. Smith}

Vellozia grao-mogulensis L. B. Smith, Contr. U.S. Nat. Herb., 35:286, pl. 11: figs. $46-48,1962$.

TyPe.-On sandstone, Serra Grão Mogul, north base of mountain, Minas Gerais, Brazil, 600-700 $\mathrm{m}$ alt, 16 August 1960, Maguire, Magalhães \& Maguire 49218 (US, holotype; NY, isotype).

Distribution.-Grão Mogul, Minas Gerais, Brazil.

\section{Vellozia squalida Martius ex Schultes $\mathrm{f}$.}

Vellozia squalida Martius ex Schultes f. in Roemer \& Schultes, Syst., 7:292, 1826.-Seubert in Martius, Fl. Bras., 3(1):83, 1847.-L. B. Smith, Contr. U.S. Nat. Herb., 35:261, 1962.Ayensu, Smithsonian Contr. Bot., 15:38, pl. 29a-c, 1974.

Type.-Without locality, Minas Gerais, Brazil, Martius s $n$ (M, holotype; F, photo 18983).

Distribution.-Santa Barbara and Conceição in the Serra do Cipó, Minas Gerais, Brazil.

\section{Vellozia coronata L. B. Smith}

Vellozia coronata L. B. Smith, Contr. U.S. Nat. Herb., 35:288, pl. 11: figs. 52-54, 1962.

TYPE.-On sandstone outcrop, ca. $20 \mathrm{~km}$ from Conceição on the road to Diamantina, Minas Gerais, Brazil, 9 August 1960, Maguire, Magalhães \& Maguire 49114 (US, holotype; NY, isotype).

Distribution.-Brazil: Minas Gerais: Conceição do Mato Dentro, Gouveia.

\section{Vellozia brevifolia Seubert}

Vellozia brevifolia Seubert in Martius, Fl. Bras., 3(1):84, pl. 10: fig. 2, 1847.-L. B. Smith, Contr. U.S. Nat. Herb., 35: $262,1962$. 56a. Vellozia brevifolia var. brevifolia

Figure 26

Vellozia brevifolia Seubert var. brevifolia.-L. B. Smith, Phytologia, 8:512, 1963.

Leaf-blades ca. $3 \mathrm{~cm}$ long, $5 \mathrm{~mm}$ wide.

Leaf Anatomy (Sellow $s \quad n$ ).-Surface View: Hairs: few tufts present on both surfaces. Epidermis: cells square to rectangular on both surfaces; thin-walled. Stomata: paracytic, $21 \times 9 \mu \mathrm{m}$; mostly in abaxial furrows; few on abaxial surface.

Transverse Section of Lamina: Dorsiventral; widely V-shaped with median adaxial groove. Adaxial surface slightly ridged; abaxial furrowed $1 / 2$ thickness of blade. Epidermis: cells rounded to dome-shaped; few conical; thin-walled. Subjacent to adaxial epidermis is 4-layered thin-walled cells interspersed with sclerenchyma strands. Below this is a layer of larger, thin-walled parenchyma cells. Subjacent to abaxial epidermis is a layer of thinwalled cells interspersed with few fibers. Sclerenchyma fiber bundles present in abaxial corners of furrows. Cuticle: thin and smooth on both surfaces. Stomata: mostly present in abaxial furrows; few on abaxial surface; flush with epidermis and surrounded by projections from furrow walls; sub-

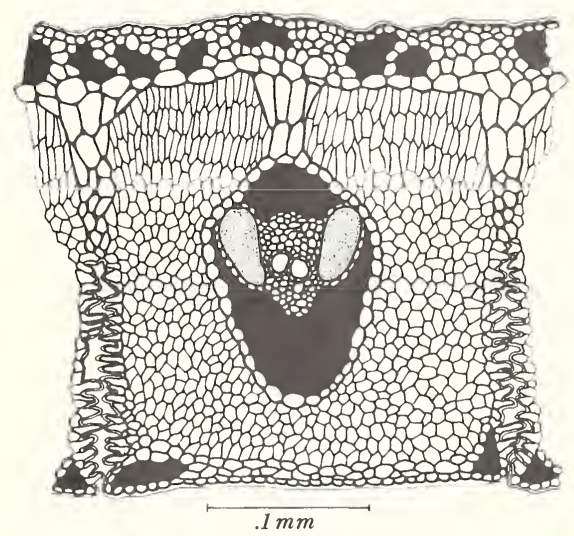

Figure 26.-Vellozia brevifolia var. brevifolia (Sellow $s$ ). 
stomatal chamber present. Mesophyll: 3 layers of palisade tissue grading into compactly arranged spongy cells. Two or three layers of large, translucent palisade cells radially arranged above vascular bundles, furrows, and midvein. Vascular bundles: 20; commissural bundles not observed. One or two large vessels present in each vascular bundle. Two phloem units lying laterally in flanges of thick, V- or Y-shaped abaxial girder. Adaxial cap present on each vascular bundle. Each vascular bundle surrounded by a bundle sheath. Crystals: none observed. Tannins: few present.

Type--Without locality, Brazil, Sellow s $n$ (B, holotype; US, photo).

Distribution.-Brazil: Minas Gerais: Lagoa Santa to Conceição do Mato Dentro and Jaboticatubas in the Serra do Cipó.

56b. Vellozia brevifolia var. angustior L. B. Smith

Vellozia brevifolia var. angustior L. B. Smith, Phytologia 8:512, figs. $12,13,1963$.

Type--Serra da Lapa (Cipó), Minas Gerais, Brazil, November 1824, Riedel s $n$ (K, holotype; US, photo).

Distribution.-Known only from the typecollection.

\section{Vellozia pilosa Goethart \& Henrard}

FIGURE 27

Vellozia pilosa Goethart \& Henrard, Blumea, 2:377, 1937.L. B. Smith, Contr. U.S. Nat. Herb., 35:262, 1962.-Ayensu, Smithsonian Contr. Bot., 15:35, pl. 43e, 1974.

Type.-On rocks, Diamantina, Minas Gerais, Brazil, 16 April 1892, Glaziou 19933 (L, holotype; $\mathrm{B}$, photo; $\mathbf{P}$, isotype).

Distribution.-Known only from the typecollection.

\section{Vellozia barbata Goethart \& Henrard}

Vellozia barbata Goethart \& Henrard, Blumea, 2:366, 1937.L. B. Smith, Contr. U.S. Nat. Herb., 35:262, 1962.

TYPE.-Rocky campo, Diamantina, Minas Gerais, Brazil, 11 April 1892, Glaziou 19942 (L, holotype; B, photo).
Distribution.-Known only from the typecollection.

\section{Vellozia streptophylla L. B. Smith}

Vellozia streptophylla L. B. Smith, Contr. U.S. Nat. Herb., 35:262, pl. 2, pl. 9: figs. 2, 3, 1962.

TYPE.-Vicinity of Diamantina, Minas Gerais, Brazil, 1840, Gardner 5233 (NY, holotype; US, photo; BM, isotype).

Distribution.-Known only from the typecollection.

\section{Vellozia declinans Goethart \& Henrard}

Vellozia declinans Goethart \& Henrard, Blumea, 2:369, 1937. -L. B. Smith, Contr. U.S. Nat. Herb., 35:262, 1962.Ayensu, Smithsonian Contr. Bot., 15:20, pl. 36d, 1974.

Vellozia leptopetala Goethart \& Henrard, Blumea, 2:374, 1937.-L. B. Smith, Contr. U.S. Nat. Herb., 35:262, 1962.Ayensu, Smithsonian Contr. Bot., 15:30, fig. 10d-f, pl. 30d-f, 1974 [in part, as to Gardner 5230, Maguire 49184, not as to type].

TyPE.-On rock, Serra da Lapa (Cipó), Minas Gerais, Brazil, November 1824, Langsdorff 1655 e. p. (LE, holotype; B, photo).

Distribution.-Brazil: Minas Gerais: Conceição do Mato Dentro, Diamantina, Gouvêia.

Note: This species might better be placed in subkey I as the trichomes are scarcely glandular, but the error was discovered too late to change the system.

\section{Vellozia marcescens L. B. Smith}

Vellozia marcescens L. B. Smith, Contr. U.S. Nat. Herb., $35: 289,1962$.

\section{1a. Vellozia marcescens var. marcescens}

Vellozia marcescens var. marcescens L. B. Smith, Contr. U.S. Nat. Herb., 35, pl. 11: figs. 55-57, 1962.

Caudex branched. Leaf-blades to $7 \mathrm{~cm}$ long, 7 $\mathrm{mm}$ wide. Scapes $15-20 \mathrm{~mm}$ long above the sheaths.

Type.-On sandstone, slopes and summit of Serra Grão Mogul, Minas Gerais, Brazil, 900-1100 m alt, 17 August 1960, Maguire, Magalhães \& Maguire 49259 (US, holotype; NY, isotype). 

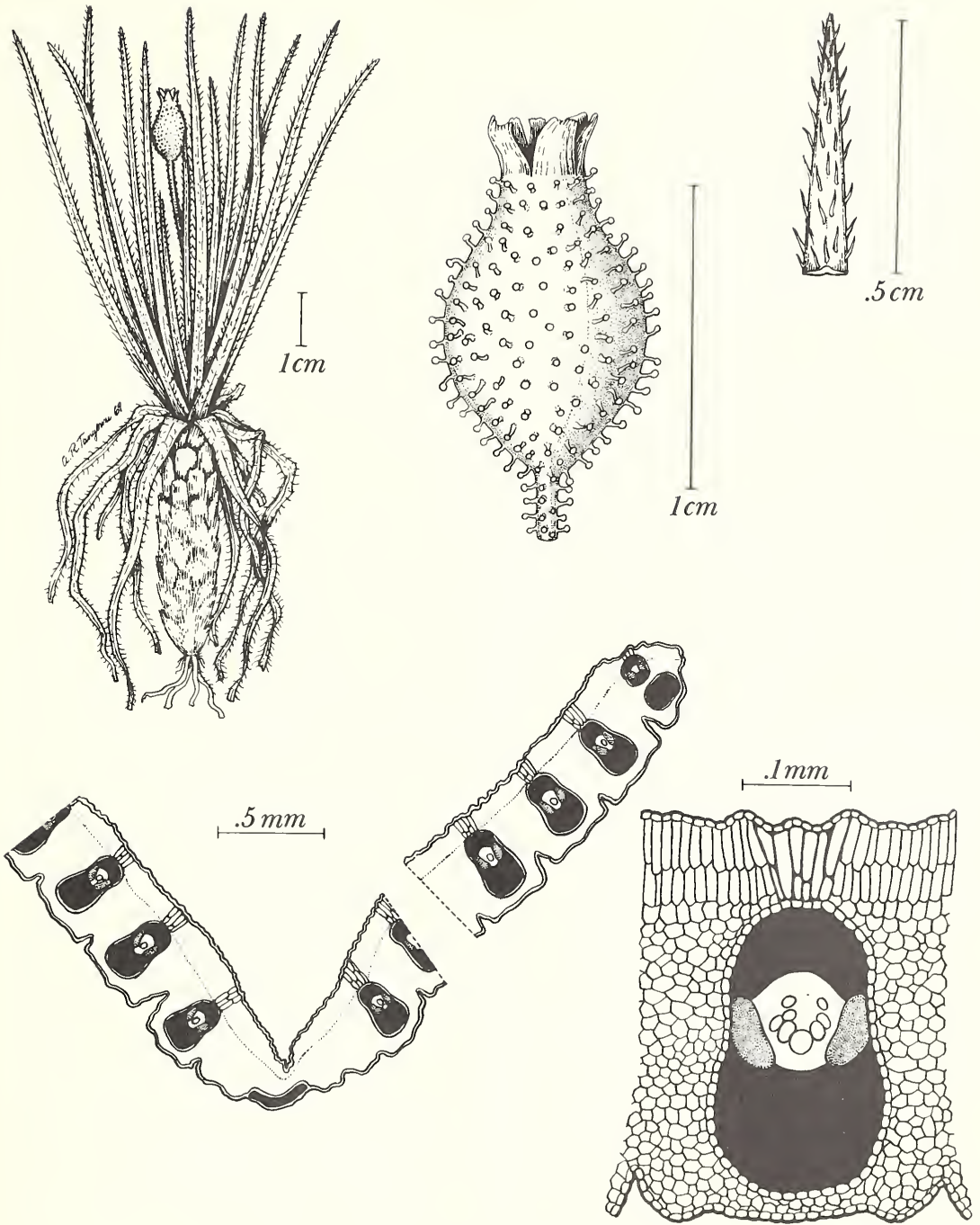

Figure 27.-Vellozia pilosa Goethart \& Henrard (Glaziou 19933). 
Distribution.-Brazil: Minas Gerais: Conceição do Mato Dentro and Grão Mogul.

\section{1b. Vellozia marcescens var. minor L. B. Smith}

Vellozia marcescens var. minor L. B. Smith, Contr. U.S. Nat. Herb., 35:289, 1962.

Caudex simple. Leaf blades smaller. Scapes larger.

TYPE.-On sandstone, Serra Grão Mogul, north base of mountain, Minas Gerais, Brazil, 600-700 m alt, 16 August 1960, Maguire, Magalhães \& Maguire 49219 (US, holotype; NY, isotype).

Distribution.-Known only from the typecollection.

\section{Vellozia spiralis $\mathrm{L}$. B. Smith}

Vellozia spiralis L. B. Smith, Contr. U.S. Nat. Herb., 35:287, pl. 11: figs. 49-51, 1962.

TYPE.-On sandstone, slopes and summit of Grão Mogul, Minas Gerais, Brazil, 900-1100 m alt, 17 August 1960, Maguire, Magalhães \& Maguire 49261 (US, holotype; NY, isotype).

Distribution.-Brazil: Minas Gerais: Barão de Cocais and Grão Mogul.

\section{Vellozia epidendroides Martius ex Schultes $\mathrm{f}$.}

Vellozia epidendroides Martius ex Schultes f. in Roemer \& Schultes, Syst., 7:292, 1826.--Seubert in Martius, Fl. Bras., 3(1):81, 1847.-L. B. Smith, Contr. U.S. Nat. Herb., 35:262, 1962.-Ayensu, Smithsonian Contr. Bot., 15:21, fig. 9a-c, pl. $31 \mathrm{~b}-\mathrm{d}, 1974$.

Vellozia gracilis Seubert in Martius, Fl. Bras., 3(1):81, pl. 9, 1847.-L. B. Smith, Contr. U.S. Nat. Herb., 35:267, 1962 [type: without locality, Minas Gerais, Brazil, Sellow $s \quad n$ (B, holotype)].

Type.-Without locality, Minas Gerais, Brazil, Martius s $n$ (M, holotype; F, photo 18973).

Distribution.-Brazil: Minas Gerais: Datas, Diamantina, Gouvêia, Jaboticatubas and Sêrro in the Serra do Cipó.

\section{Vellozia leptopetala Goethart \& Henrard}

Figure 28

Vellozia leptopetala Goethart \& Henrard, Blumea, 2:374, 1937.-L. B. Smith, Contr. U.S. Nat. Herb., 35:262, 1962. Vellozia epidendroides Martius ex Schultes f. var. major
L. B. Smith, Contr. U.S. Nat. Herb., 35:288, 1962 [type: on rock exposures, summit of Serra do Cipó, along road from Hotel Chapeu do Sol, km 120-140, Minas Gerais, Brazil, 1200-1300 $\mathrm{m}$ alt., 8 August 1960, Maguire, Magalhães \& Maguire 49098 (NY, US)].

Vellozia epidendroides Martius ex Schultes f. var. divaricata L. B. Smith, Contr. U.S. Nat. Herb., 35:289, 1962 [type: on rocks, sandstone terraces and ridges, summit of Serra do Cipó, km 112-128 along road from Hotel Chapeu do Sol, Minas Gerais, Brazil, 1200-1300 m alt., August 1960, Maguire, Magalhães \& Maguire 49064 (NY)].

Vellozia confusa L. B. Smith \& Ayensu [nomen.; based on Maguire, Magalhães \& Maguire 49098 and Hatschbach, Smith \& Ayensu 28800].

Leaf ANatomy (Hatschbach, Smith o Ayensu 28800; Maguire, Magalhães \& Maguire 49098).Surface View: Hairs: few tufts observed on both surfaces. Epidermis: cells on both surfaces square to rectangular, thin-walled. Stomata: tetracytic, $21 \times 15 \mu \mathrm{m}$; present in abaxial furrows only.

Transverse Section of Lamina: Dorsiventral; widely V-shaped with small median adaxial groove. Adaxial surface slightly undulating; abaxial surface furrowed $1 / 2$ thickness of blade. Epidermis: cells on both surfaces rounded to dome-shaped; few conical; thin-walled. Subjacent to adaxial epidermis is $\mathbf{2}$ or 3 layers of thin-walled, parenchyma cells interspersed with sclerenchyma fiber bundles. Below this is a layer of large, thin-walled, parenchyma cells. Subjacent to abaxial epidermis is a layer of sclerenchyma fibers interspersed with few thin-walled cells, followed by a layer of small thin-walled parenchyma. Cuticle: slightly thickened and ridged on both surfaces. Stomata: present in abaxial furrows; raised slightly above surface and associated with many projections from furrow walls; small substomatal chamber present. Mesophyll: 5 or 6 layers of small palisade cells followed by compactly arranged rounded cells. Large translucent palisade cells radially arranged above vascular bundles, furrows, and midvein. Vascular bundles: 27 (19 in Maguire 49098); commissural bundles not observed. One to three large vessels present in each bundle. Two phloem units lying laterally in flanges of U-shaped abaxial girder. Adaxial cap present on all vascular bundles. Each vascular bundle surrounded by a bundle sheath. Crystals: none observed. Tannins: few present.

Note: Although the leaf of Maguire 49098 is smaller than Hatschbach, Smith of Ayensu 28800, the specimens are identical in their anatomy. 


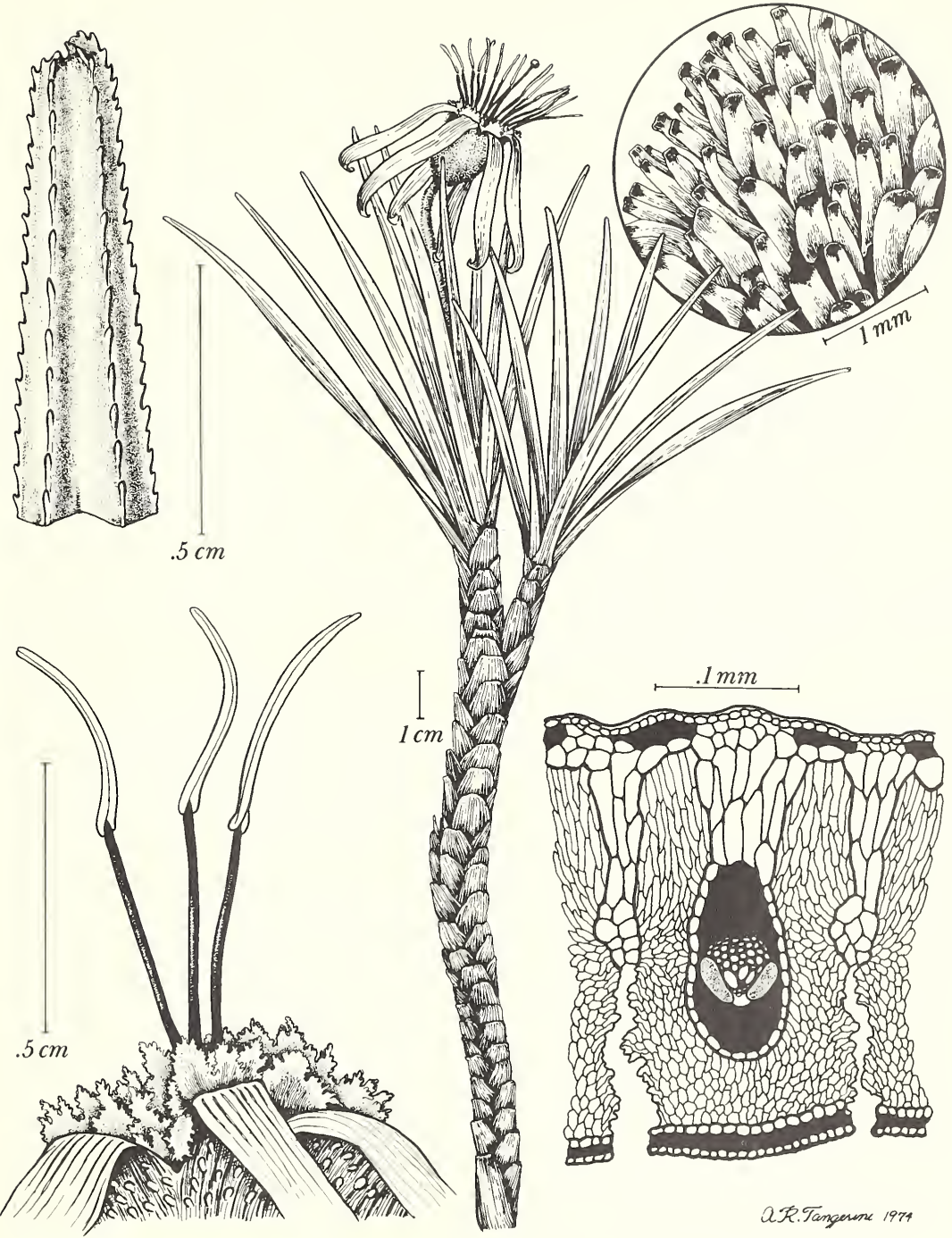

Figure 28.-Vellozia leptopetala Goethart \& Henrard (Menezes, L. B. Smith \& Ayensu 35). 

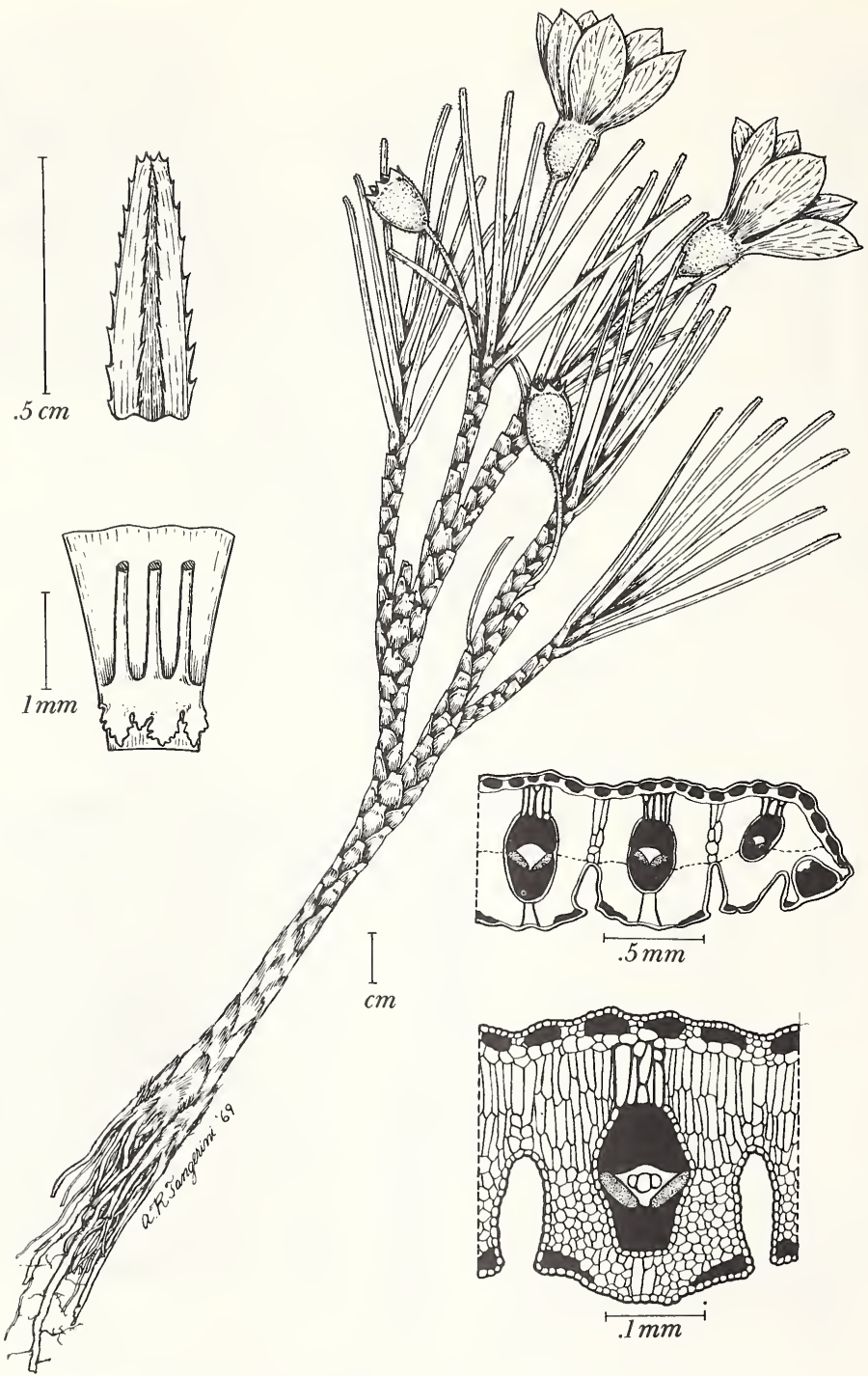

Figure 29,-Vellozia variegata Goethart \& Henrard (Santos Lima \& Brade 14288). 
TyPE.-Serra da Lapa (Gipó), Minas Gerais, Langsdorff s $n$ (L, holotype; B, photo).

Distribution.-Brazil: Minas Gerais: Summit of Serra do Cipó, Maguire, Magalhães \& Maguire 49098 (NY, US). On rock and sandy soil, km 35, Chapeu de Sol to Conceição do Mato Dentro, 4 July 1969, Menezes \& Sazima 35 (SPF, US). On rock, Fazenda Palmeira, Jaboticatubas, Serra do Cipó, 18 January 1972, Hatschbach, Smith \& Ayensu 28800 (MBM, US).

\section{Vellozia variegata Goethart \& Henrard}

\section{Figure 29}

Vellozia variegata Goethart \& Henrard, Blumea, 2:381, 1937. Vellozia magdalenae L. B. Smith \& Ayensu in Ayensu, Smithsonian Contr. Bot., 15:31, pls. 14a, 43b, 1974 [nomen.; based on Santos Lima \& Brade 13298 and 14288 (RB)].

TyPE.-Pedra do Conego, Nova Friburgo, Rio de Janeiro, Brazil, 23 June 1880, Glaziou 12221 (L, holotype; B, photo).

Distribution.-Brazil: Espírito Santo: Castelo, Rio de Janeiro: Nova Friburgo, Santa Maria Madalena. Minas Gerais: Carangola.

\section{Subkey III}

\section{Vellozia flavicans Martius ex Schultes f.}

Vellozia flavicans Martius ex Schultes f. in Roemer \& Schultes, Syst., 7:293, 1826.-Seubert in Martius, Fl. Bras., 3(1):78, 1847.-L. B. Smith, Contr. U.S. Nat. Herb., 35:264, 1962.Ayensu, Smithsonian Contr. Bot., 15:23, figs. 3k-1, 11a-c, pl. 3le-g, 1974.

Vellozia squamata Pohl, Pl. Bras., 1:124, pl. 99, 1827 [type: sandy and rocky summits, Serra Dourada and Engenho de São Izidro (Isidoro near Goiás?), Goiás, Brazil, March 1819, Pohl 1270 (M, holotype; F, photo 18984)].

TYPE.-Without locality, Minas Gerais, Brazil, Martius s $n$ (M, holotype; F, photo 18974).

Distribution.-Brazil: Bahia: Barreiras. Minas Gerais: Diamantina, Itambé(?). Goiás: Anápolis, Chapada da Contagem, Formosa, Veadeiros. Distrito Federal: Brasilia, Gama, Sobradinho. Mato Grosso: Rio Turvo, Rondonópolis.

\section{Vellozia glauca Pohl}

Vellozia glauca Pohl, Pl. Bras., 1:125, pl. 100, 1827.-Seubert in Martius, Fl. Bras., 3(1):79, 1847.-L. B. Smith, Contr.
U.S. Nat. Herb., 35:264, 1962.-Ayensu, Smithsonian Contr. Bot., 15:25, figs. 3e-f, $11 \mathrm{~d}-\mathrm{f}, 1974$.

Vellozia glauca var. genuina Seubert in Martius, Fl. Bras., $3(1): 79,1847$.

Vellozia hamosa Pohl ex Seubert in Martius, Fl. Bras., 3(1):79, 1847 [nomen].

TyPe.-Sandy summits, near Aldeia Carretao de Pedro Terceiro, Goiás, Brazil, Pohl s $n$ (M, holotype; $\mathrm{n} v$ ).

Distribution.-Brazil: Goiás: Alto de Paraíso, Caiaponia, Cristalina, Guará, Veadeiros.

\section{Vellozia glochidea Poh1}

Vellozia glochidea Pohl, Pl. Bras., 1:129, 1827.-Seubert in Martius, Fl. Bras., 3(1):79, 1847,-L. B. Smith, Contr. U.S. Nat. Herb., 35:264, 1962.-Ayensu, Smithsonian Contr. Bot., 15:25, fig. 12a-c, pls. 5a, 31h, 32a-b, 1974.

Vellozia gardneri Goethart \& Henrard, Blumea, 2:371, 1937 [type: Natividade, Goiás, Brazil, Gardner 4018 (P, US, W)].

TYPE.-Sandy slopes, Serra Dourada, Goiás, Brazil, Pohl s n (M, holotype; F, photo 18975).

Distribution.-Brazil: Pará: Serra dos Carajás, Marabá. Minas Gerais: São João da Chapada. Goiás: Corumbá de Goiás, Goiania, Luziania. Pirenópolis, Posse, Serra Dourada.

\section{Vellozia nuda L. B. Smith \& Ayensu, new species}

Figure 30

A V. glochidea Pohl, cui valde affinis, caudice graciliore, foliorum vaginis apice multo expositis differt.

Caudex simple or few-branched, $1 \mathrm{~m}$ high, 8-12 $\mathrm{mm}$ in diameter (including leaf-sheaths). Leaves few-ranked; sheaths subdensely imbricate with 2 cm or more of the apex exposed, even, glabrous, nonglutinous; blades linear, attenuate, $15-50 \mathrm{~cm}$ long, 4-9 $\mathrm{mm}$ wide, serrulate on the thick yellow margins, otherwise even and glabrous.

Scapes terminal, single, erect, 3-15 cm long (above the leaf-sheaths), terete, even, glabrous. Ovary cylindric, $1 \mathrm{~cm}$ long at anthesis (type) to $3 \mathrm{~cm}$ in fruit, covered with subulate trichomes. Tepals narrowly elliptic, $45 \mathrm{~mm}$ long, violet (!Hatschbach). Stamens ca. 18, ca. $25 \mathrm{~mm}$ long; phalanges unappendaged. Style exceeding the stamens; stigmas orbicular, terminal, spreading. 


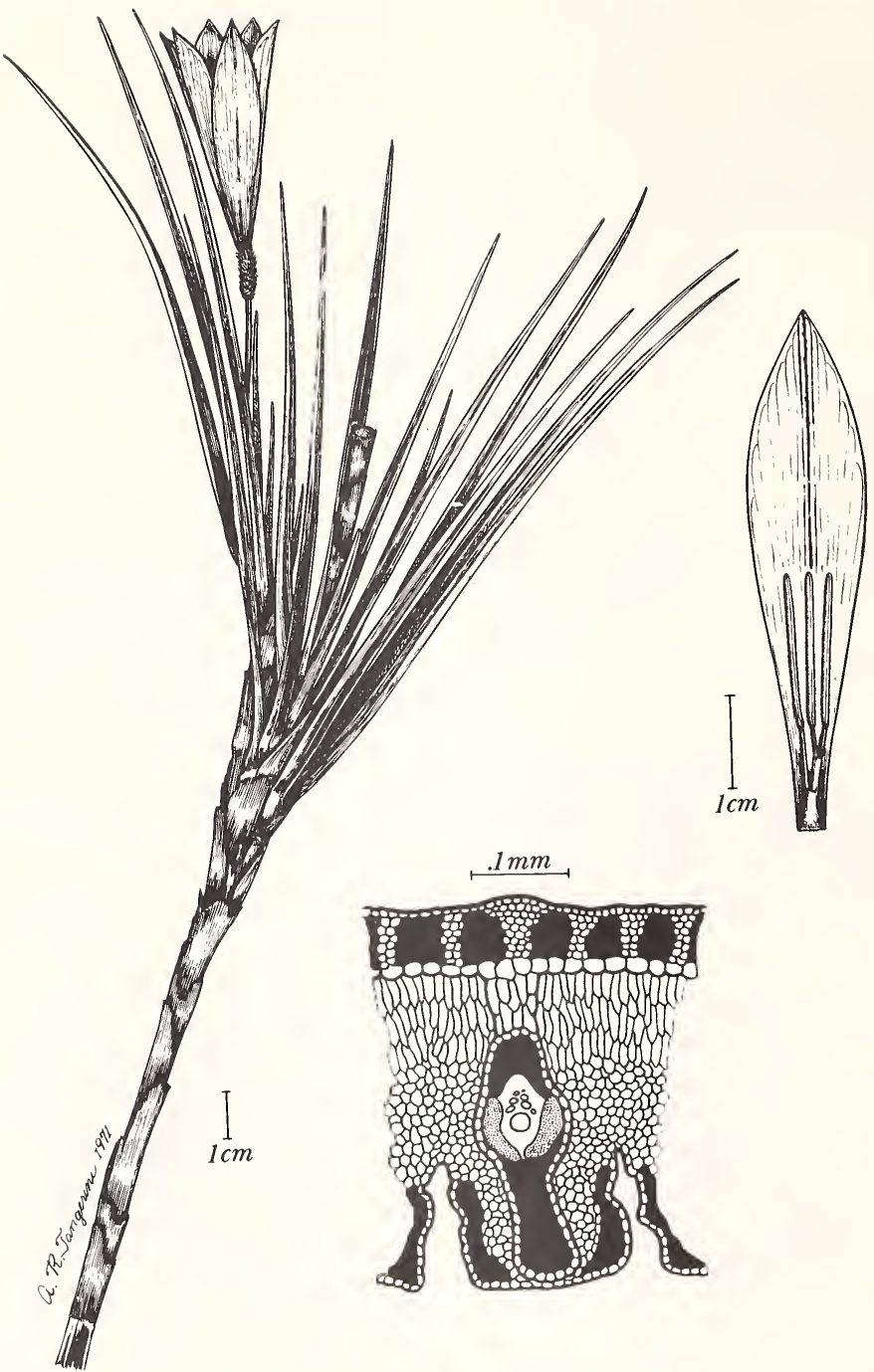

Figure 30.-Vellozia nuda L. B. Smith \& Ayensu, new species (Hatschbach \& Pelanda 28029). 
Leaf Anatomy.-Ayensu, Smithsonian Contr. Bot., 15:33, fig. 4c, pl. 37e, 1974.

TYPE.-Sandy soil at base of rock outcrop, Rio Jequitinhonha, Mun. Cunha Magalhães, Minas Gerais, Brazil, 16 November 1971, Hatschbach b Pelanda 28029 (US, holotype; MBM, isotype).

Distribution.-Brazil: Minas Gerais: L. B. Smith, Ayensu \& Hatschbach 16000 (US, isotype). Edge of stream, on rocks, $9 \mathrm{~km}$ by road southwest of Mendanha and the Rio "Jequiti" on the road to Diamantina, $1150 \mathrm{~m}$ alt, 14 April 1973, Anderson 8877 (NY, UB, US).

\section{Vellozia crassicaulis Martius ex Schultes f.}

Vellozia crassicaulis Martius ex Schultes f. in Roemer \& Schultes, Syst., 7:292, 1826.-L. B. Smith, Contr. U.S. Nat. Herb., 35:265, 1962.-Ayensu, Smithsonian Contr. Bot., 15:18, fig. 12d-f, pls. 11a-b, 32c-g, 33a, 1974.

Vellozia albiflora Pohl, Pl. Bras., 1:121, pl. 96, 1827.-Seubert in Martius, Fl. Bras., 3(1):82, 1847 [type: Arraial da Nossa Senhora de Penha, Minas Gerais, Brazil, Pohl s $n$ (M, holotype; F, photo 18968)].

Vellozia circinans Goethart \& Henrard, Blumea, 2:367, 1937. -L. B. Smith, Contr. U.S. Nat. Herb., 35:266, 1962 [type: among rocks, Serra de São João del Rei, Minas Gerais, Brazil, Glaziou 17291 (L, P)].

TyPE.-Without locality, Minas Gerais, Brazil, Clausen s $n$ (NY, isotype?).

Distribution.-Brazil: Minas Gerais: Barão de Cocais, Conceição de Mato Dentro, Diamantina, Gouvêa, Jaboticatubas, Mendanha, Sêrro. Goiás: Cristalina, Goiás Velho, Niquelândia, Pirenópolis, Rio Corumba.

\section{Vellozia sulphurea Pohl}

FIgURE 31

Vellozia sulphurea Pohl, Pl. Bras., 1:120, pl. 95, 1827.Seubert in Martius, Fl. Bras., 3(1):82, 1847.-L. B. Smith, Contr. U.S. Nat, Herb., 35:265, 1962.

Leaf Anatomy (Pohl s n).--Surface View: Hairs: few tufts present on both surfaces. Epidermis: cells on both surfaces square to rectangular; thin-walled. Stomata: tetracytic, $21 \times 15 \mu \mathrm{m}$; present in abaxial furrows only.

Transverse Section of Lamina: Dorsiventral; widely V-shaped with median adaxial groove and margins turned downward. Adaxial surface ridged;

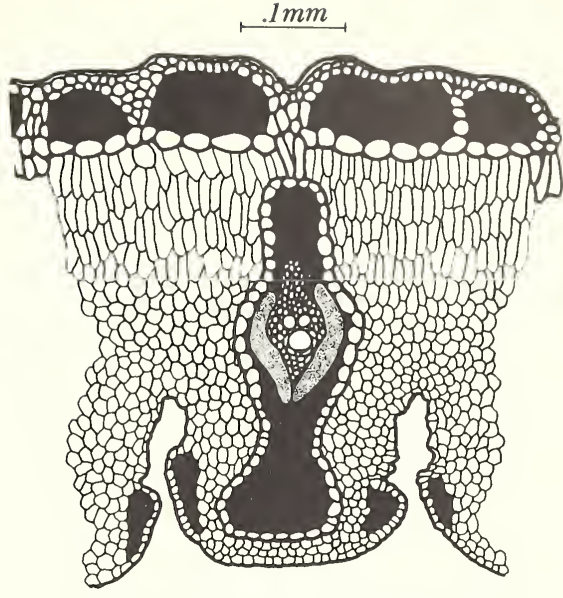

Figure 31.-Vellozia sulphurea Pohl (Pohls n).

abaxial furrowed $1 / 4$ to $1 / 3$ thickness of blade. Epidermis: cells square to rectangular on both surfaces; few crescent-shaped; thin-walled. Adaxial cells replaced with sclerenchyma fibers in many places. Subjacent to adaxial epidermis is 2 or 3 layers of thin-walled cells interspersed with large sclerenchyma fiber bundles of 3 or 4 layers. Below is a layer of large, thin-walled parenchyma cells. Cuticle: thick and slightly ridged on entire surface. Stomata: present in abaxial furrows only; flush with epidermal surface; small substomatal chamber present. Mesophyll: 3 or 4 layers of palisade cells followed by compactly arranged spongy tissue. One or two layers of large, translucent cells radially arranged above vascular bundles; 3 or 4 above midvein. Vascular bundles: 23; commissural bundles not observed. One or two large vessels present in each bundle, mostly one. Two phloem units lying laterally in flanges of thin, Y-shaped abaxial girder. Thin, partial adaxial girder present on each bundle. Bundle sheath completely surrounding each vascular bundle. Crystals: cuboidal, present in bundle sheath and in abaxial epidermal cells. Tannins: few present.

TYPE.-Dry rocky ground, near Arraial São João Baptista, Rio das Mortes, between Tapanhoacanga 


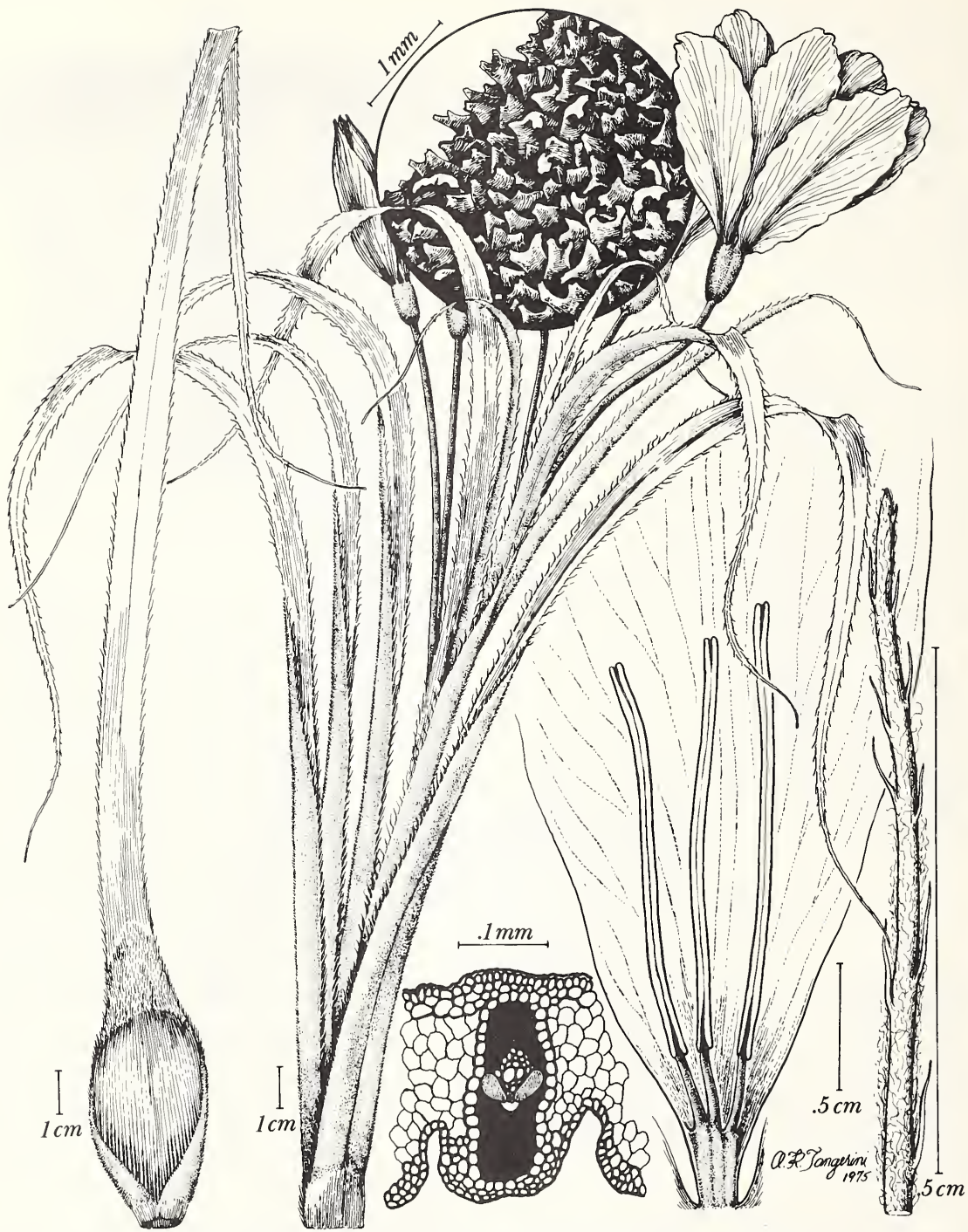

Figure 32.-Vellozia nivea L. B. Smith \& Ayensu, new species (Joly \& Semir 3167). 
and Padre Bento, Minas Gerais, Brazil, Pohl s $n$ (M, holotype; F, photo 18985 - leaves only).

Distribution.-Known only from the typecollection.

\section{Vellozia nivea L. B. Smith \& Ayensu, new species}

Figure 32

A $V$. seubertiana Goethart \& Henrard et $V$. froesii L. B. Smith, cuibus affinis, foliorum laminis subtus dense albo-tomentosis, ovario squamis erosis obtecto differt.

Caudex ca. $5 \mathrm{dm}$ high (!Joly), stout (judging by leaf-bases). Leaves with sheaths $5 \mathrm{~cm}$ long, yellow, densely white-tomentose outside toward apex, glabrous within; blades very narrowly triangular, filiform-attenuate, to $36 \mathrm{~cm}$ long, $15 \mathrm{~mm}$ wide at base, flat becoming inrolled on drying, densely white-tomentose beneath and at base above, margins pectinate-serrate with fine suberect $6 \mathrm{~mm}$ long trichomes.

Scapes terminal, 6, erect, over $20 \mathrm{~cm}$ long, minutely white-pubescent. Ovary ellipsoid, $13 \mathrm{~mm}$ long, subterete, covered with erect, erose whitish scales. Tepals elliptic, $55 \mathrm{~mm}$ long, violet, slightly lepidote at base and medianly. Stamens ca. 18, ca. $2 \mathrm{~cm}$ long, phalanges unappendaged. Style exceeding the stamens; stigmas terminal, orbicular spreading.

Leaf Anatomy (Joly \& Semir 3167).-Surface View: Hairs: single, conical hairs present on abaxial surface only. Epidermis: cells on both surfaces rectangular; thin-walled. Stomata: paracytic, some tetracytic, $27 \times 15 \mu \mathrm{m}$; present mainly in abaxial furrows; few on adaxial and abaxial surfaces.

Transverse Section of Lamina: Dorsiventral; very widely $\mathrm{V}$-shaped with almost entire margin turned down. Trichomes present on abaxial surface and in abaxial furrows. Adaxial surface almost smooth; abaxial surface furrowed $1 / 3$ to $1 / 2$ thickness of blade. Epidermis: cells on both surfaces rounded to dome-shaped; thin-walled. Subjacent to adaxial epidermis is 2 or 3 layers of rounded, thin-walled parenchyma cells. Cuticle: slightly thickened and smooth on both surfaces. Stomata: present on abaxial surface and in abaxial furrows; stomata flush with epidermal surface; substomatal chamber present. Mesophyll: very large, rounded, translucent cells present above furrows. There is little spongy mesophyll lateral to furrows. Most of mesophyll totally obliterated. Vascular bundles: 53; commissural bundles not observed. One or two large vessels present in each bundle. Two phloem units lying laterally in flanges of U-shaped abaxial girder. Adaxial cap present on each bundle. Each vascular bundle surrounded by a bundle sheath. Crystals: none observed. Tannins: few present.

Note: The adaxial sclerenchyma cap is highly extended thus approaching the sclerenchyma pattern found in Barbacenia. The extreme obliteration of the mesophyll is quite distinctive.

TYPE.-Sandy soil between rocks, km 142 of the route from Lagoa Santa to Conceição do Mato Dentro, Minas Gerais, Brazil, 21 August 1972, Joly \& Semir 3167 (US, holotype; SPF, isotype).

Distribution.-Known only from the typelocality.

\section{Vellozia seubertiana Goethart \& Henrard}

\section{FIGURE 33}

Vellozia seubertiana Goethart \& Henrard, Blumea, 2:380, 1937.-L. B. Smith, Contr. U.S. Nat. Herb., 35:265, 1962 [nom. nov., stat. nov.]

Vellozia glauca Pohl var. cujabensis Seubert in Martius, F1. Bras., 3(1):79, 1847.-L. B. Smith, Contr. U.S. Nat. Herb., $35: 264,1962$.

Leaf ANatomy (Anderson 6854; Harley \& Barroso 11339; Harley \& Lima 11500; Hatschbach 31959; Hatschbach o Koczicki 33161; Malme 1738-C; Manso in Lhotsky 90).-Surface View: Hairs: many, small tufts on adaxial surface of Anderson 6854, Harley \& Barroso 11339 and Harley \& Lima 11500; few large tufts on Hatschbach \& Koczicki 33161; none on adaxial of the rest. Abaxial surface of Anderson 6854 and Harley \& Barroso 11339 has many, small tufts; Harley \& Lima 11500 has none; Hatschbach 31959 and Hatschbach o Koczicki 33161 have few large tufts and Malme 1738-C and Manso in Lhotsky 90 have few small tufts of hairs. Epidermis: cells on both surfaces in all collections are square to rectangular with thin walls; few 6-sided cells present. Stomata: paracytic, some tetracytic, $23 \times 15 \mu \mathrm{m}$ on adaxial and $18 \times 15 \mu \mathrm{m}$ on abaxial; most adaxial stomata slightly larger than abaxial ones; all but Anderson 6854 and Harley of Barroso 11339 had adaxial 


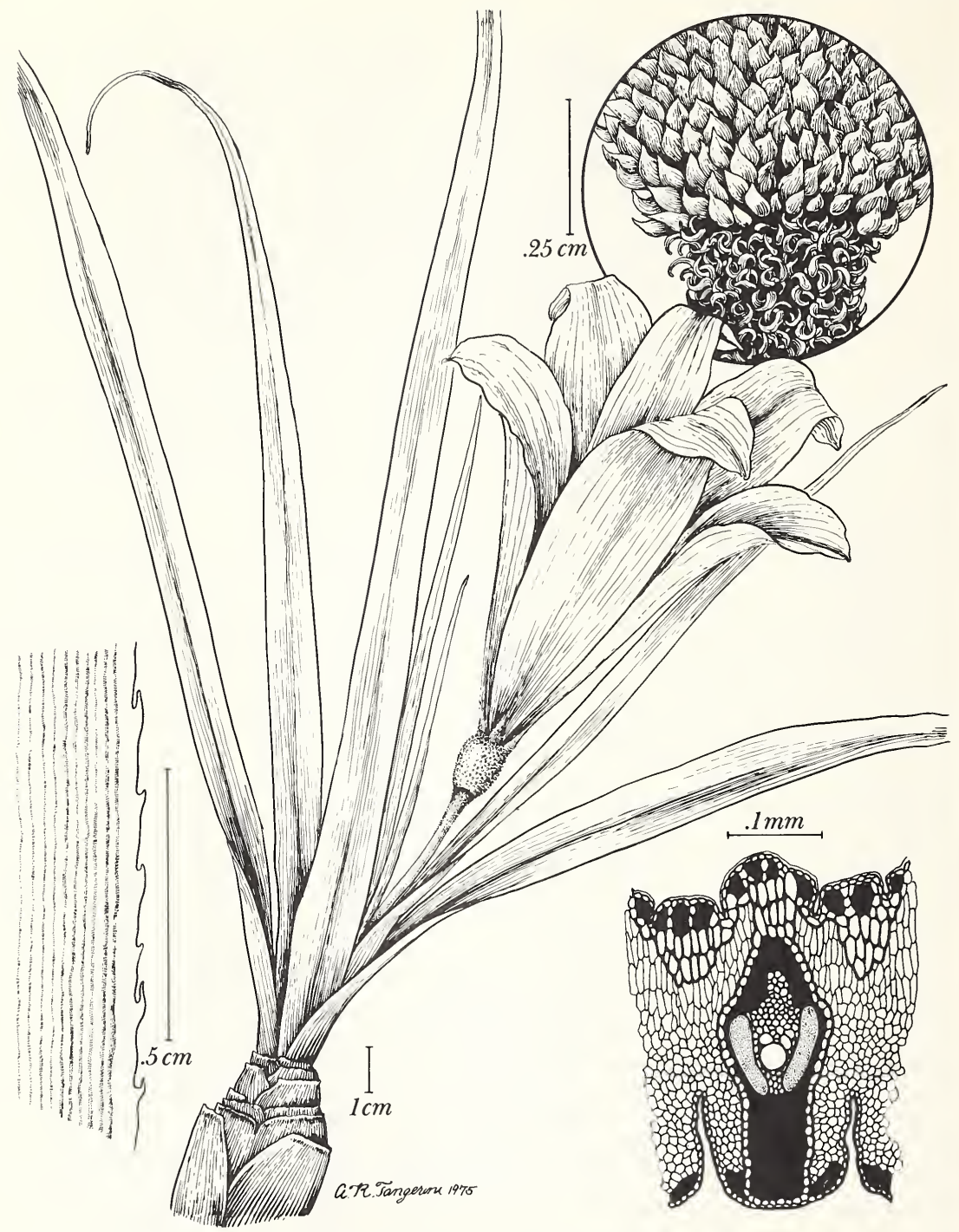

Figure 33.-Vellozia seubertiana Goethart \& Henrard (Manso in Lhotsky 90). 
stomata and all specimens had stomata in abaxial furrows; very few noticed on abaxial surface.

Transverse Section of Lamina: Dorsiventral; $A n$ derson 6854 and Harley of Barroso 11339 widely V-shaped; adaxial surface smooth; abaxial furrowed $1 / 4$ thickness of blade; rest are deeply V-shaped; adaxial surface slightly to very ridged; abaxial furrowed $1 / 4$ thickness of blade. Abaxial hairs mainly tufts, observed on all but Harley \& Lima 11500; adaxial tufts only observed on Harley \& Barroso 11339. Epidermis: adaxial cells rounded to square or dome-shaped on most; crescent-shaped on Harley \& Barroso 11339 and Hatschbach \& Koczicki 33161. Abaxial cells rounded to dome-shaped. All cells thin-walled. Subjacent to adaxial epidermis (except Harley o Lima 11500) is 4 or 5 layers of sclerenchyma fiber bundles interspersed with 2 or 3 rows of 3- or 4-layered parenchyma cells followed by one layer of distinct parenchyma cells. (Harley \& Lima 11500 has 1 or 2 layers of parenchyma interspersed with 1 or 2 layers of sclerenchyma fiber bundles; 1 layer of parenchyma subjacent to it.) These layers are broken up by mesophyll in Malme 1738-C and Manso in Lhotsky 90; continuous in rest. Subjacent to abaxial epidermis is a layer of sclerenchyma fiber bundles followed by another layer of parenchyma in all except Anderson 6854, which has 5 or 6 layers of sclerenchyma fiber bundles. Cuticle: adaxial cuticle thin and smooth to slightly ridged except in Anderson 6854 where it is thick. Abaxial cuticle thin and very ridged with conical projections in all but Malme 1738-C and Manso in Lhotsky 90, where it is smooth. Stomata: present on adaxial surface of all specimens except Anderson 6854 and Harley \& Barroso 11339; stomata present in abaxial furrows of all specimens. Stomata flush with surface with substomatal chamber present. Few papillae in Anderson 6854. Mesophyll: 4 or 5 layers of palisade tissue followed by compactly arranged spongy cells. One to four layers of translucent cells radially arranged above vascular bundles, furrows, and midvein. Sclerenchyma fiber bundles at abaxial corners of furrows. Vascular bundles: 15-45; commissural bundles observed. One to three large vessels present in each bundle, mostly one. Two phloem units lying laterally in flanges of Y-shaped abaxial girder. Adaxial cap present on all bundles. Bundle sheath completely surrounding all bundles. Crystals: present in mesophyll and bundle sheath of all ex- cept Hatschbach \& Koczicki 33161. Tannins: few present.

TyPE.-Cuiabá, Rio Cuiabá, Mato Grosso, Brazil, Manso in Lhotsky 90 (B, holotype; US, photo).

Distribution.-Brazil: Goiás: among sandstone rocks at summit of serra, Serra Dourada near Goiás, 18 December 1968, Harley \& Barroso 11339 (K). Woodland margin, Pirineus, 26 December 1968, Harley \& Lima 11500 (K). Rocky slope, $8 \mathrm{~km}$ by road west of Monte Alegre, 600-700 m, 11 March 1973, Anderson 6854 (NY, UB, US).

Mato Grosso: In cerrado, Buriti in Serra da Chapada, 27 June 1894, Malme 1738-C (S). Sandstone outcrops, Rio Verde between Campo Grande and Cuiabá, 15 May 1973, Hatschbach 31959 (MBM, US). Sandstone outcrops in cerrado, by route BR 163, Rio Verde, 13 November 1973, Hatschbach \& Kocziki 33161 (MBM, US).

\section{Vellozia froesii L. B. Smith}

Vellozia froesii L. B. Smith, Contr. U.S. Nat. Herb., 35:265, pl. 3, 1962.

Type.-Mucugê, Serra da Sincorá, Bahia, Brazil, February 1943, Fróes 19980 (IAN, holotype; NY, isotype).

Distribution.-Known only from the typecollection.

\section{Vellozia hypoxoides $\mathbf{L}$. B. Smith}

Vellozia hypoxoides L. B. Smith, Contr. U.S. Nat. Herb., 35: 265, pl. 9: fig. 8, 1962.-Ayensu, Smithsonian Contr. Bot., 15:28, pls. $37 \mathrm{c}, 50 \mathrm{a}, 1974$.

Type.-Serra dos Pirineus, Mun. Corumbá, Goiás, Brazil, 17 February 1956, Macedo 4330 (US, holotype; IAN, isotype).

Distribútion.-Known only from the typelocality.

\section{Vellozia blanchetiana L. B. Smith}

Vellozia blanchetiana L. B. Smith, Phytologia, 8:513, figs. 18, $19,1963$.

TyPE.-Serra Assuruá, near Rio São Francisco, Bahia, Brazil, 1828, Blanchet 2814 (K, holotype; S, US, isotypes). 


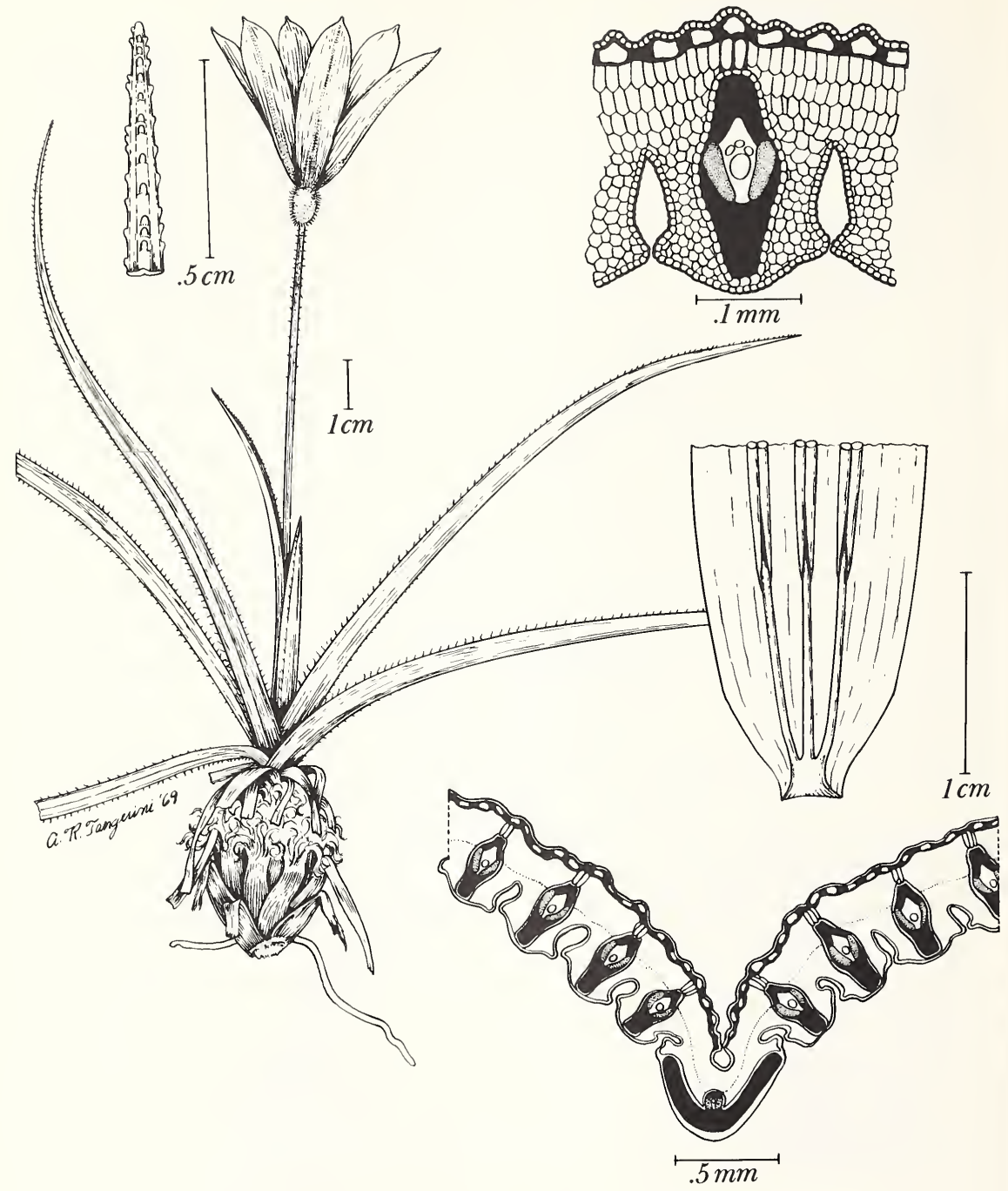

Figure 34.-Vellozia crinita Goethart \& Henrard (Glaziou 16388). 
Distribution.-Known only from the typecollection.

\section{Vellozia bulbosa L. B. Smith}

Vellozia bulbosa L. B. Smith, Contr. U.S. Nat. Herb., 35:266, pl. 4, 1962.

TyPE.-On rocks in savanna, Serra do Cachimbo, Pará, Brazil, $425 \mathrm{~m}$ alt., 17 December 1956, Pires, Black, Wurdack \& Silva 6423 (IAN, holotype).

Distribution.-Known only from the typecollection.

\section{Vellozia crinita Goethart \& Henrard}

\section{FIGURE 34}

Vellozia crinita Goethart \& Henrard, Blumea, 2:368, 1937.L. B. Smith, Contr. U.S. Nat. Herb., 35:267, 1962.-Ayensu, Smithsonian Contr. Bot., 15:18, pl. 42d, 1974.

TYPE.-On rocks, summit of São José (João?) d'El Rey, Minas Gerais, Brazil, 15 December 1886, Glaziou 16388 (L, holotype; P, isotype).

Distribution.-Known only from the typecollection.

\section{Vellozia swallenii L. B. Smith}

Vellozia swallenii L. B. Smith, Contr. U.S. Nat. Herb., 35: 265, pl. 9: figs. 9, 10, 1962.-Ayensu, Smithsonian Contr. Bot., 15:39, fig. 13a-b, pl. 33b-c, 1974 .

TYPE.-On chapada (dry brushy field), between Barra do Corda and Grajaú, Maranhão, Brazil, 1-5 March 1934, Swallen 3617 (US, holotype).

Distributıon.-Brazil: Maranhão: Barra do Corda to Grajaú. Goiás: Niquelândia, Serra Dourada, Vianópolis. Distrito Federal: Brasília.

\section{Vellozia pumila Goethart \& Henrard}

\section{FIGURE 35}

Vellozia pumila Goethart \& Henrard, Blumea, 2:378, 1937.L. B. Smith, Contr. U.S. Nat. Herb., 35:265, 1962.-Ayensu, Smithsonian Contr. Bot., 15:37, pls. 5d, 44b, 1974.

Vellozia glaziovii Goethart \& Henrard, Blumea, 2:372, 1937 [type: in campo, near Ponso, Serra dos Veadeiros, Goiás, Brazil, 6 January 1895, Glaziou 22214 (L, P, US, photo)].

TyPE.-Near cascade in campo, Ponte Alta,
Goiás, Brazil, 28 September 1894, Glaziou 22215 (L, holotype; B, photo; $\mathrm{P}$, isotype).

Distribution.-Brazil: Goiás: Corumbá de Goiás, Ponte Alta, Serra Dourada, Veadeiros. Distrito Federal: Gama.

\section{Vellozia pulchra L. B. Smith}

Vellozia pulchra L. B. Smith, Contr. U.S. Nat. Herb., 35:290, pl. 12: figs. 60-62, 1962.

Type-_Granite slopes, immediately west of Pedra Azul, Minas Gerais, Brazil, 21 August 1960, Maguire, Magalhães \& Maguire 49289 (US, holotype; NY, isotype).

Distribution.-Known only from the typecollection.

\section{Vellozia bahiana L. B. Smith \& Ayensu, new species}

Figure 36

A $V$. cinerascente Martius ex Schultes f., cui affinis, foliorum trichomatibus simplicibus filiformibusque, alteris ovarii simplicibus subulatisque differt.

Caudex largely unknown, apically $12 \mathrm{~mm}$ in diameter (including leaf-sheaths). Leaves fewranked, densely white-puberulent on all exposed surfaces, nonglutinous; sheaths subdensely imbricate with $15 \mathrm{~mm}$ of the apex exposed, $4 \mathrm{~cm}$ long, the midnerve contrasting, the line of abscission strongly arched; blades linear, attenuate to a narrowly obtuse apex, $28 \mathrm{~cm}$ long, $10 \mathrm{~mm}$ wide at base, the margins and midnerve laxly serrulate with erect trichomes slightly larger and stouter than the others.

Scapes (detached) terminal(?), 2(?), to $12 \mathrm{~cm}$ long, sulcate, glabrous. Ovary subglobose, $8 \mathrm{~mm}$ long, covered with contorted subulate acute or bidentate trichomes. Tepals elliptic, 5-6 cm long. Stamens ca. 18 , over $3 \mathrm{~cm}$ long; phalanges irregular, unappendaged. Style $4 \mathrm{~cm}$ long; stigmas terminal, orbicular, spreading.

Leaf Anatomy (Maia s $n$ in SP 110129).-Surface View: Hairs: tufts of hairs present on both surfaces; single hairs also present on abaxial, especially in furrows. Epidermis: cells on both surfaces square 


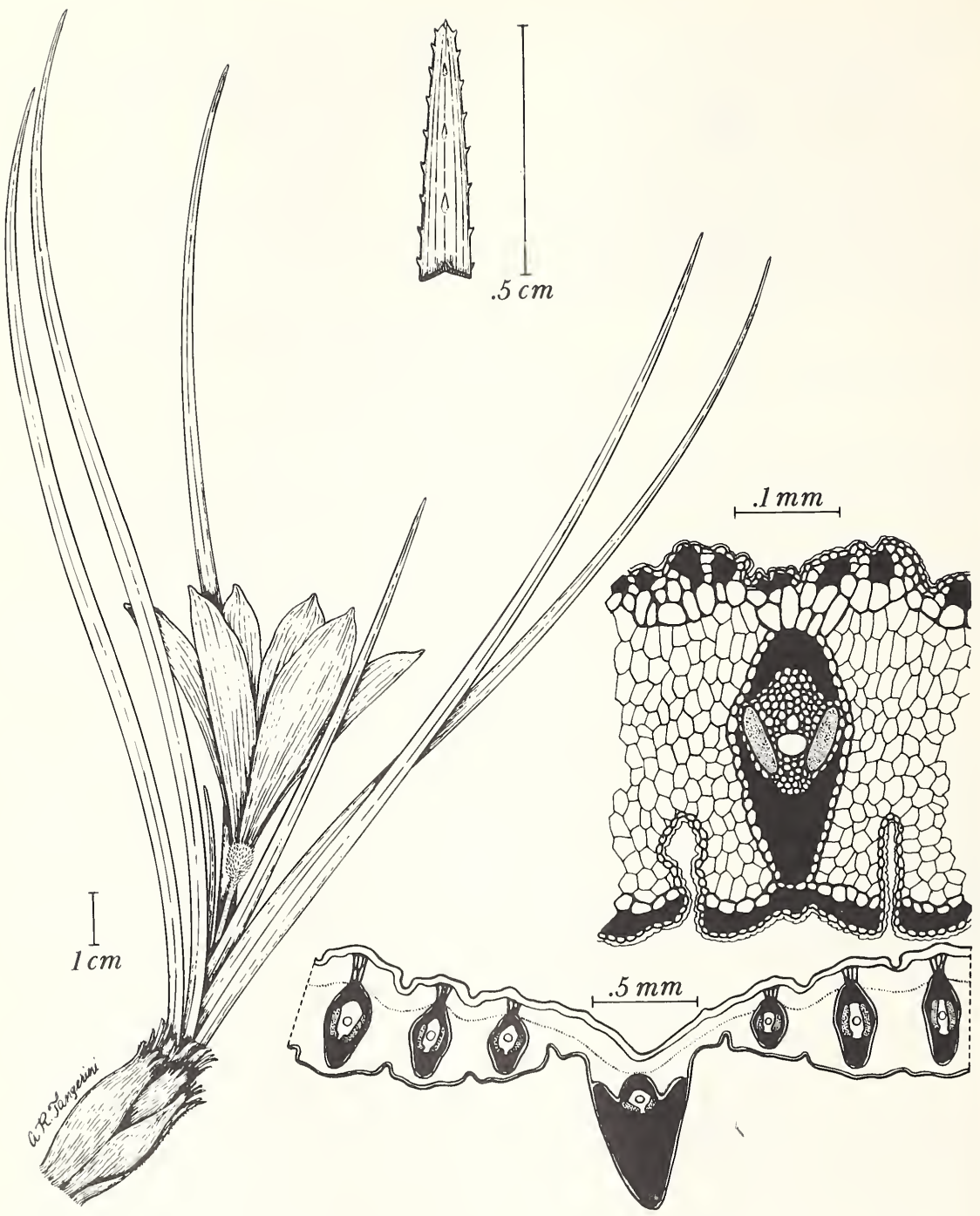

Figure 35.-Vellozia pumila Goethart \& Henrard (Glaziou 22215). 


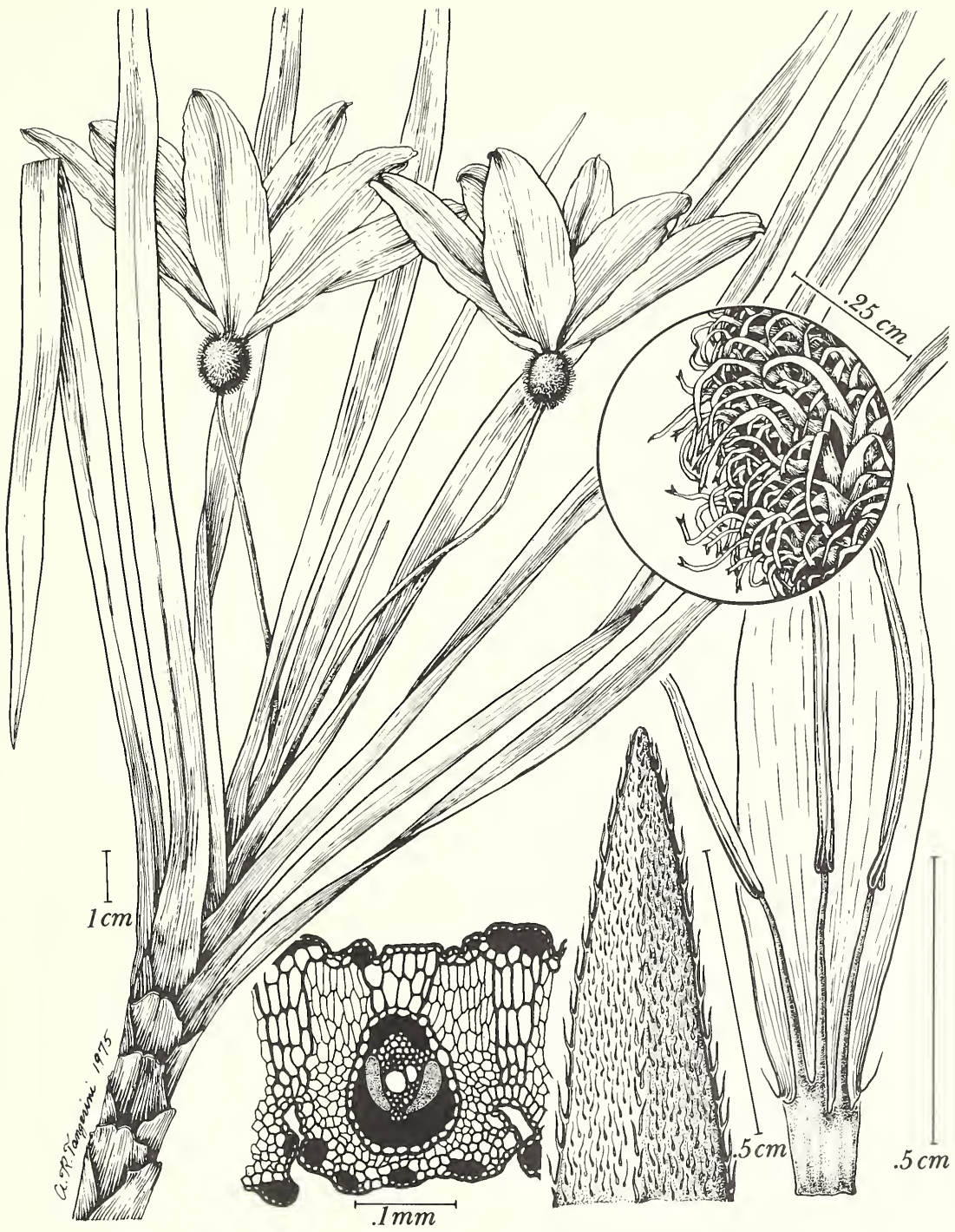

Figure 36.-Vellozia bahiana L. B. Smith \& Ayensu, new species (Maia in SP 110129). 


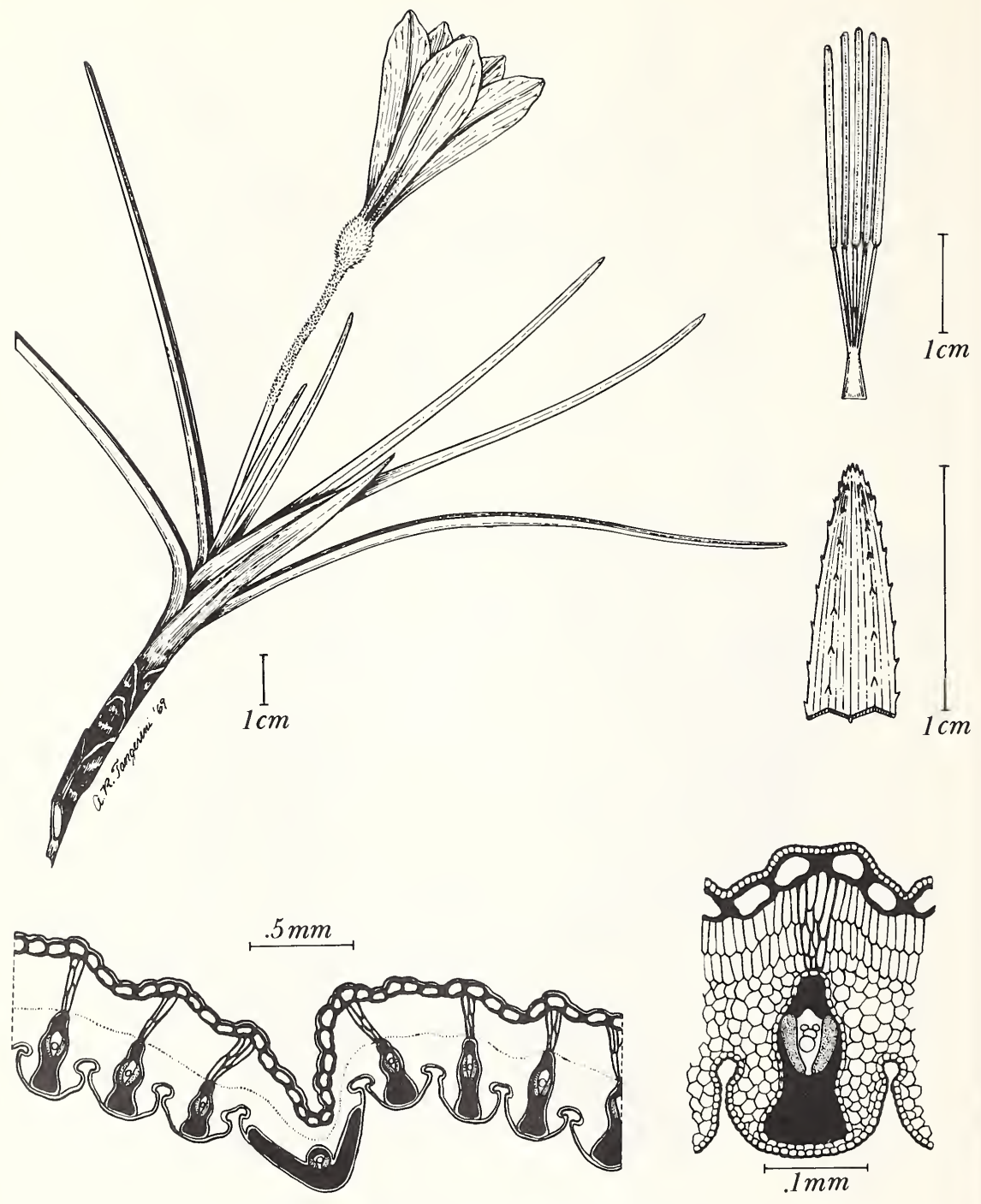

Figure 37.-Vellozia dasypus Seubert (Blanchet 3558). 
to rectangular; thin-walled. Stomata: tetracytic, $21 \times 18 \mu \mathrm{m}$; present in rows on adaxial surface and in abaxial furrows.

Transverse Section of Lamina: Dorsiventral; widely V-shaped; almost horizontal. Adaxial surface furrowed one-fourth thickness of blade. Hairs present on both surfaces. Epidermis: cells on both surfaces rounded to dome-shaped; few conical; thinwalled; interspersed with sclerenchyma strands. Subjacent to both epidermises is a layer of thinwalled parenchyma cells. Cuticle: thickened and ridged on adaxial; thicker and ridged on abaxial. Stomata: present on adaxial surface and in abaxial furrows; stomata flush with surface; substomatal chamber present. Mesophyll: 3 or 4 layers palisade tissue followed by compact spongy tissue; 1 or 2 layers translucent palisade radially arranged above vascular bundles, furrows, and midvein. Sclerenchyma fibers at abaxial corners of furrows. Vascular bundles: 35; commissural bundles observed; 1 or 2 large vessels present in each bundle, mostly one. Two phloem units lying laterally in flanges of $\mathrm{Y}$ - or U-shaped abaxial girder. Adaxial cap present on each bundle. Bundle sheath completely surrounding each bundle. Crystals: present in bundle sheath. Tannins: few present.

Note: This species is one of few in the family that exhibit adaxial stomata complexes.

Type-Without exact locality, Bahia, Brazil, W. Maia s $n$ (SP 110129, holotype; US, photo).

Distribution.-Unknown.

\section{Vellozia cinerascens (Martius ex Schultes f.) Martius ex Seubert}

Vellozia cinerascens (Martius ex Schultes f.) Martius ex Seubert in Martius, FI. Bras., 3(1):74, 1847,-L. B. Smith, Contr. U.S. Nat. Herb., 35:266, 1962.-Ayensu, Smithsonian Contr. Bot., 15:16, fig. 3c-d, pls. 4c-d, 42c, 1974.

Xerophyta cinerascens Martius ex Schultes f. in Roemer \& Schultes, Syst., 7:292, 1826.

TYPE.-Rocky fields near Fazenda Capoculo, [Pernambuco] and in grassy campo of Santa Isabella, [Piauí], Brazil, April 1819, Martius s n (M, holotype; F, photo 18971).

Distribution.-Piauí, Pernambuco: Bahia: Serra de Tinga, Sentocé (Sento Se), $9^{\circ} 38^{\prime} \mathrm{S}, 41^{\circ} 12^{\prime} \mathrm{W}$, Zehntner 323 (RB).

\section{Vellozia dasypus Seubert}

Figure 37

Vellozia dasypus Seubert in Martius, FI. Bras., 3(1):81, 1847.L. B. Smith, Contr. U.S. Nat. Herb., 35:266, 1962.-Ayensu, Smithsonian Contr. Bot., 15:20, fig. 13c-e, pls. 16a, 33d-h, 34a, 1974.

Type.-Jacobina, Bahia, Brazil, Blanchet 3558 (G, holotype; F, photo 25077).

Distribution.-Brazil: Sergipe: Serra de Itabaiana. Bahia: Maraú, Mucugê (Serra de Sincorá), São Salvador.

\section{Vellozia scabrosa L. B. Smith \& Ayensu, new species}

Figure 38

A $V$. exilis Goethart \& Henrard atque $V$. grisea Goethart \& Henrard, quibus affinis, caudice robusto, scapis elongatis differt.

Caudex branched, $2 \mathrm{~m}$ high (!Hatschbach), 12 $\mathrm{mm}$ thick at apex (including leaf-sheaths), appearing much thicker because of the short erect branches. Leaves ca. 10-ranked, glabrous; sheaths densely imbricate with apex slightly exposed; blades of uncertain duration because old leaves badly burned, linear, attenuate to an oblique obtuse apex, to $14 \mathrm{~cm}$ long, $8 \mathrm{~mm}$ wide at base, flat, the thickened margin very finely serrulate toward base, elsewhere entire.

Scapes terminal, 2-4, erect, to $8 \mathrm{~cm}$ long (above the leaf-sheaths), densely scabrous. Ovary obovoid, $11 \mathrm{~mm}$ long, densely scabrous; epigynous tube short. Tepals elliptic, $3 \mathrm{~cm}$ long, violet (!Hatschbach). Stamens ca. 18; phalanges lacerate-appendaged.

LEAF ANATOMY (Hatschbach 29970).-Surface View: Hairs: none observed. Epidermis: cells on both surfaces square to rectangular; thin-walled. Stomata: tetracytic, $18 \times 9 \mu \mathrm{m}$ on abaxial, slightly shorter and wider on adaxial; stomata in rows on adaxial surface and in abaxial furrows.

Transverse Section of Lamina: Dorsiventral; widely V-shaped with small median adaxial groove. Adaxial surface slightly ridged; abaxial furrowed $1 / 3$ to $1 / 2$ thickness of blade. Epidermis: cells on both surfaces square to rectangular and crescent-shaped; thin-walled. Subjacent to adaxial epidermis in some places are 1-3 layers of sclerenchyma fiber bundles; there is a layer of small, thin-walled parenchyma 


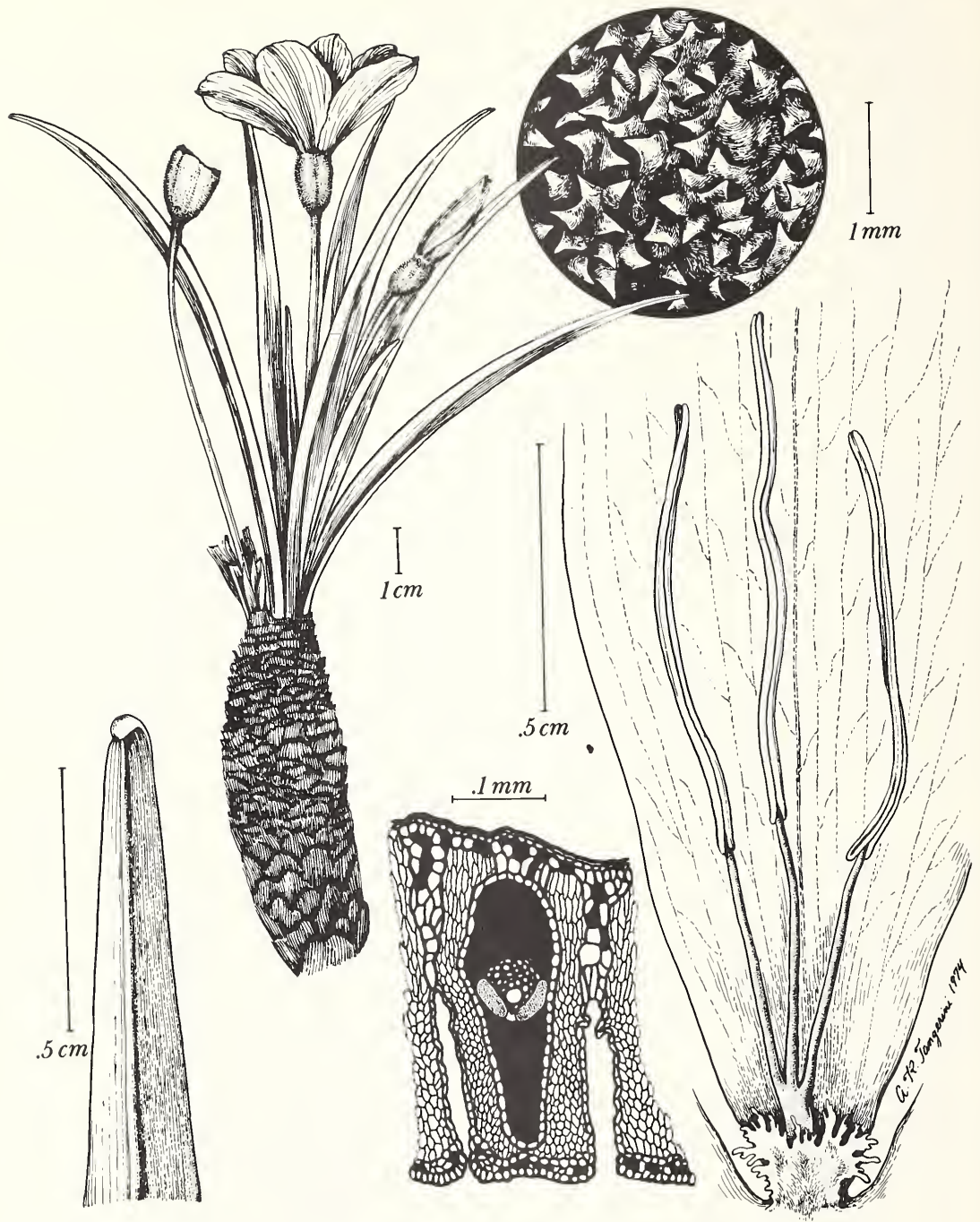

Figure 38.-Vellozia scabrosa L. B. Smith \& Ayensu, new species (Hatschbach 29970). 
below epidermis; sclerenchyma fiber bundles occur below this layer followed by another parenchyma layer subjacent to the bundles. Similar distribution of sclerenchyma and parenchyma occur on abaxial side, except that sclerenchyma is one layer thick. Cuticle: thick and slightly ridged on adaxial surface; thick and very ridged with cone-like projections on abaxial surface. Stomata: present on adaxial surface and in abaxial furrows; stomata flush with epidermal surface; small substomatal chamber present. Mesophyll: 3 or 4 layers of palisade tissue followed by compactly arranged cells. Three or four layers of large translucent cells radially arranged above furrows and tangentially on vascular bundles; mesophyll above midvein almost entirely translucent. Vascular bundles: 33; few commissural bundles observed. One or two large vessels in each bundle, mostly one. Two phloem units lying laterally in flanges of fairly thick, Y-shaped to deeply U-shaped abaxial girder. Large adaxial cap present on each bundle. Bundle sheath completely surrounding each vascular bundle. Crystals: none observed. Tannins: present.

TyPe.-Jaboticatubas: Rocky campo, Serra do Cipó, Minas Gerais, Brazil, 6 August 1972, Hatschbach 29970 (US, holotype; MBM, isotype).

Distribution.-Known only from the typecollection.

\section{Vellozia exilis Goethart \& Henrard}

\section{FIGURE 39}

Vellozia exilis Goethart \& Henrard, Blumea, 2:370, 1937.L. B. Smith, Contr. U.S. Nat. Herb., 35:267, 1962.-Ayensu, Smithsonian Contr. Bot., 15:23, fig. 14d-e, pl. 34e-f, 1974.

TYPE.-Between rocks, Chapadão dos Veadeiros, Goiás, Brazil, 9 January 1895, Glaziou 22220 (L, holotype; B, photo).

Distribution.-Known only from the typelocality, ca. $14^{\circ} \mathrm{S}, 47^{\circ} \mathrm{W}$.

\section{Vellozia grisea Goethart \& Henrard}

Figure 40

Vellozia grisea Goethart \& Henrard, Blumea, 2:373, 1937.L. B. Smith, Contr. U.S. Nat. Herb., 35:267, 1962.-Ayensu, Smithsonian Contr. Bot., 15:26, figs. 3i-j, 4b, pls. 16b, 42e, 50c, 1974.
Trpe.-Between Goiás, Goiás, and Cuiabá, Mato Grosso, Weddell 3006 (P, holotype; B, photo 23).

Distribution.-Known only from the typecollection.

\section{Subkey IV}

\section{Vellozia hemisphaerica Seubert}

Vellozia hemisphaerica Seubert in Martius, Fl. Bras., 3(1):80, pl. 9, 1847.-L. B. Smith, Contr. U.S. Nat. Herb., 35:261, 1962.-Ayensu, Smithsonian Contr. Bot., 15:28, fig. 8e, 1974 .

TYPE.- "Mediterraneis" (probably in the vicinity of São Salvador), Bahia, Brazil, Blanchet 2544 (G, $\mathrm{P}$, isotypes).

Distribution.-Known only from the typecollection.

89. Vellozia burle-marxii L. B. Smith \& Ayensu, new species

FIGURE 41

A $V$. hemisphaerica Seubert, cui valde affinis, caudice gracili, foliorum vaginis apice expositis differt.

Caudex decumbent, branched, over $20 \mathrm{~cm}$ long, 3-4 $\mathrm{mm}$ in diameter (including leaf-bases). Leaves 3-ranked, nonglutinous; sheaths subdensely imbricate with the apices exposed for $7 \mathrm{~mm}$, even, glabrous, whitish turning to brown with age; blades persistent, spreading from the first, contorted, linear, acute to a setiform apex, $5 \mathrm{~cm}$ long, $3 \mathrm{~mm}$ wide, complanate, laxly serrulate, otherwise even and glabrous.

Scapes solitary, becoming lateral, erect, $20 \mathrm{~cm}$ long, less than $1 \mathrm{~mm}$ in diameter, trigonous, finely and laxly stipitate-glandular. Ovary hemisphaeric, $5 \mathrm{~mm}$ in diameter, densely and finely stipitateglandular. Tepals elliptic, obtuse, $2 \mathrm{~cm}$ long, violet. Stamens 18, phalanges slenderly cylindric. Style exceeding the stamens; stigmas terminal, orbicular, spreading.

Leaf Anatomy.-Ayensu, Smithsonian Contr. Bot., 15:14, fig. 16a-c, 1974.

Type.-Pedra de Chapeu, Bahia, Brazil, cultivated 14 December 1968, Burle-Marx s $n$ (US 2537159, holotype). 

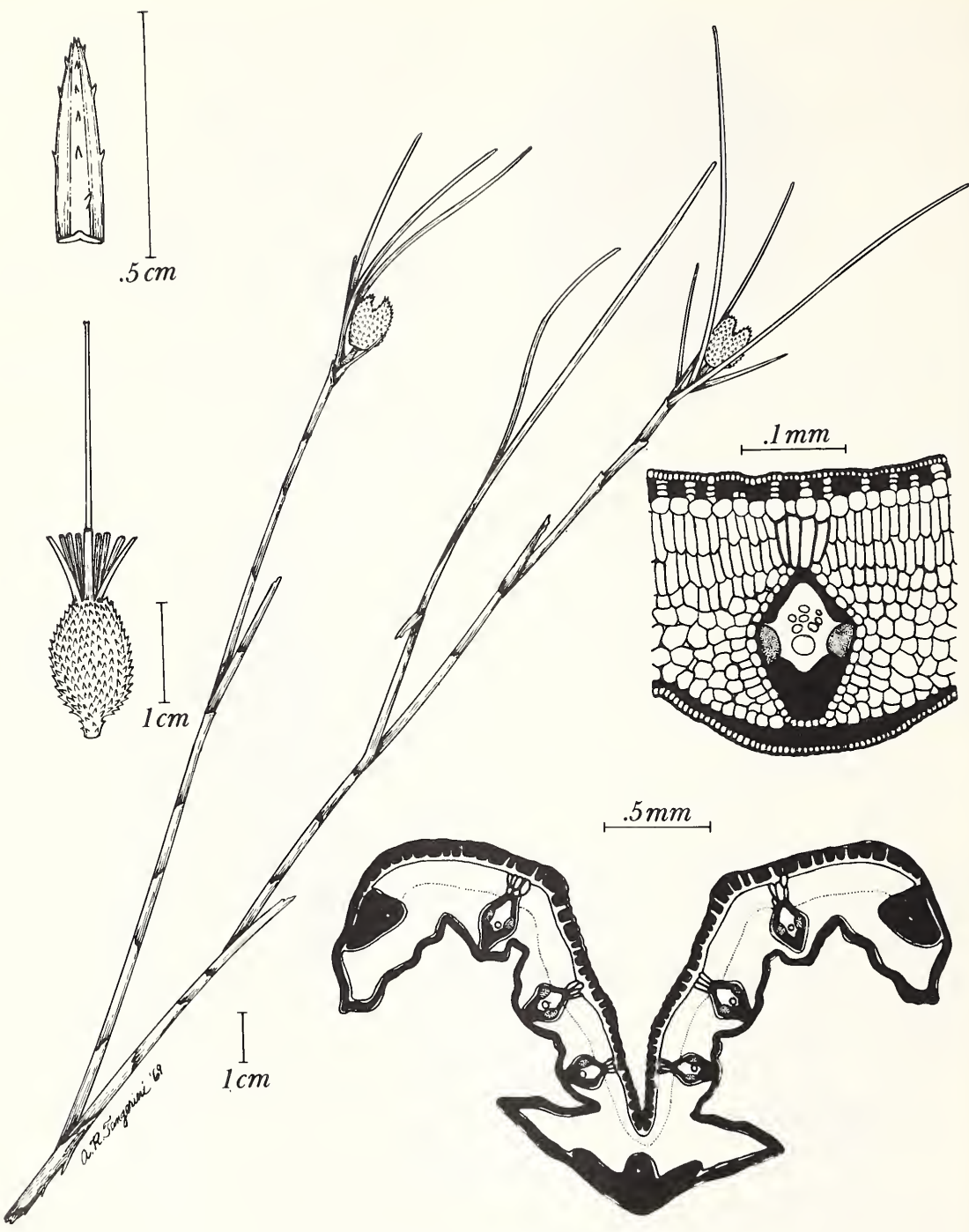

Figure 39.-Vellozia exilis Goethart \& Henrard (Irwin 12696). 


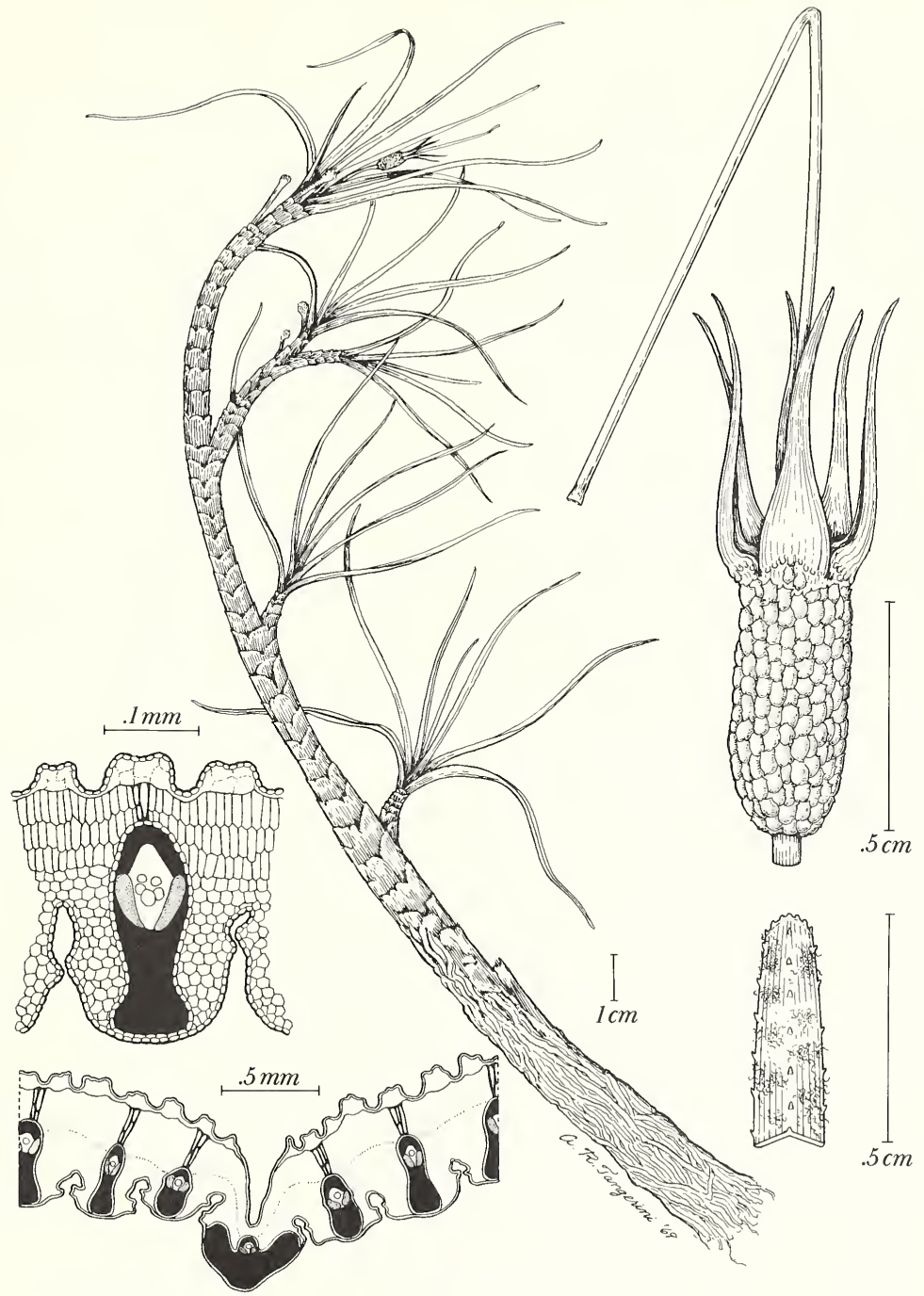

Figure 40.-Vellozia grisea Goethart \& Henrard (Weddell 3006). 


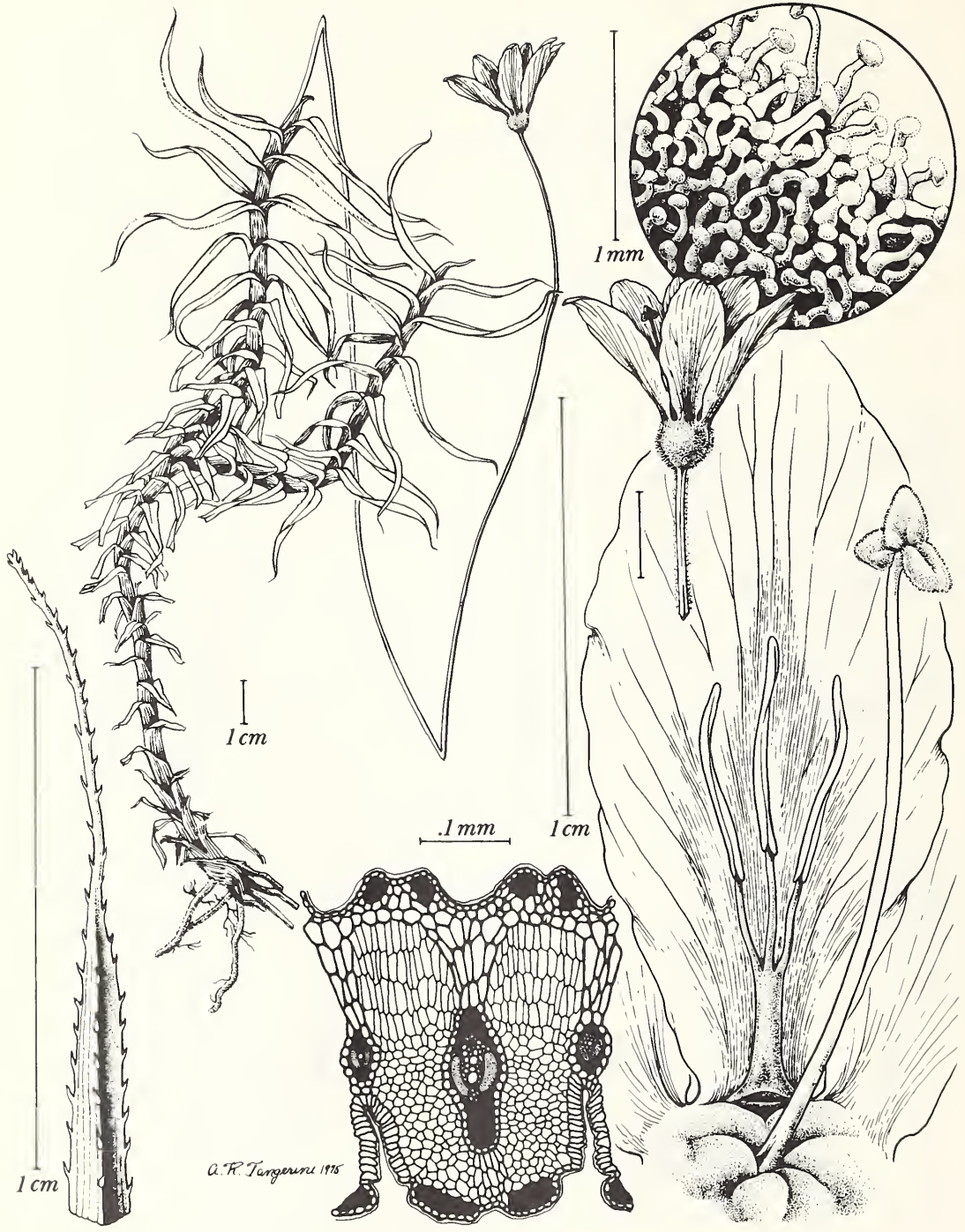

Figure 41.-Vellozia burle-marxii L. B. Smith \& Ayensu, new species (Burle-Marx $s$ ). 
Distribution.-Known only from the typecollection.

90. Vellozia lilacina L. B. Smith \& Ayensu, new species

Figure 42

A V. leptopetala Goethart \& Henrard cui affinis, tepalis divergentibus, filamentis pallidis differt.

Caudex erect, branching, $7 \mathrm{dm}$ high, $9 \mathrm{~mm}$ in diameter (including leaf-sheaths). Leaves 3-ranked; sheaths imbricate but with the apical $10 \mathrm{~mm}$ exposed, glabrous, brown; blades linear, obtuse or retuse, to $13 \mathrm{~cm}$ long, $7 \mathrm{~mm}$ wide, bicarinate toward apex, laxly serrulate on margins and keels.

Scapes becoming lateral, to $8 \mathrm{~cm}$ long (above the leaf-sheaths), $2 \mathrm{~mm}$ in diameter, laxly and finely stipitate-glandular. Ovary ellipsoid, $15 \mathrm{~mm}$ long, densely stipitate-glandular; epigynous tube short. Tepals elliptic, obtuse, $45 \mathrm{~mm}$ long, lilac (!Menezes). Stamens ca. 30; phalanges fimbriateappendaged. Style exceeding the stamens; stigmas terminal, orbicular, spreading.

Leaf ANAтомy (Menezes 238).-Surface View: Hairs: few large tufts present on both surfaces. Epidermis: cells square to rectangular on both surfaces; thin-walled. Stomata: paracytic, some tetracytic, $21 \times 15 \mu \mathrm{m}$; present in furrows and on abaxial surface.

Transverse Section of Lamina: Dorsiventral; flanges V-shaped. Adaxial surface slightly ridged; abaxial surface furrowed $1 / 4$ to $1 / 3$ thickness of blade. Epidermis: cells rounded to dome-shaped on both surfaces; few conical; thin-walled. Subjacent to adaxial epidermis is a layer of thin-walled cells interspersed with sclerenchyma fibers. No sclerenchyma above midvein. Below this is a layer of fairly large, thin-walled parenchyma cells. Cuticle: slightly thickened and smooth over entire surface. Stomata: present in abaxial furrows and on abaxial surface; stomata slightly above epidermal surface; small substomatal chamber present. Mesophyll: 1-3 layers of palisade cells followed by rounded, compactly arranged spongy mesophyll. Cells translucent above furrows. Strands of sclerenchyma fibers at abaxial corners of furrows. Vascular bundles: 2227; commissural bundles not observed. One or two vessels present in each bundle, mostly one. Two phloem units lying laterally in flanges of $\mathrm{U}$ - or
Y-shaped abaxial girder. Fairly large adaxial cap present on each bundle. Bundle sheath completely surrounding each bundle. Crystals: none observed. Tannins: few present.

TYPE.-Km 127 on the west side of the road from Lagoa Santa to Conceição do Mato Dentro, Serra do Cipó, 23 July 1972, Menezes 238 (US, holotype; $\mathrm{SPF}$, isotype).

Distribution.-Known only from the type-area.

\section{Vellozia arenicola L. B. Smith}

Vellozia arenicola L. B. Smith, Phytologia, 8:512, figs. 14, 15, 1963.

TyPE--In sand, Tijuco (Diamantina), Minas Gerais, Brazil, December 1824, Riedel s $n$ (K, holotype).

Distribution.-Known only from the typecollection.

92. Vellozia stipitata L. B. Smith \& Ayensu, new species

Figure 43

A V. echinata Goethart \& Henrard, cui affinis, foliorum laminis latioribus haud vel vix revolutis recurvatis, ovarii glandulis atris differt.

Caudex undoubtedly tall and stout, branched (!Semir \& Menezes). Leaves glabrous; sheaths $6 \mathrm{~cm}$ long, whitish; blades very narrowly triangular, longattenuate, $30 \mathrm{~cm}$ long, $17 \mathrm{~mm}$ wide, flat or nearly so, serrulate with slender suberect spines.

Scapes terminal, 4, $15 \mathrm{~cm}$ long (above the leafsheaths), $1.5 \mathrm{~mm}$ in diameter, subdensely stipitateglandular. Ovary ellipsoid, $14 \mathrm{~mm}$ long, covered with fine pale stipitate glands. Tepals elliptic, with the inner distinctly broader, $5 \mathrm{~cm}$ long, purple (!Semir \& Menezes). Stamens ca. 20; phalanges irregular, lacerate-appendaged.

Leaf Anatomy (Irwin 28419; Menezes 230; Semir of Menezes 284).-Surface View: Hairs: few tufts present on adaxial surface. Epidermis: cells on both surfaces square to rectangular; thin-walled. Stomata: paracytic, $24 \times 18 \mu \mathrm{m}$; present in abaxial furrows only.

Transverse Section of Lamina: Dorsiventral; widely V-shaped with margins turned down and small median adaxial groove. Adaxial surface 


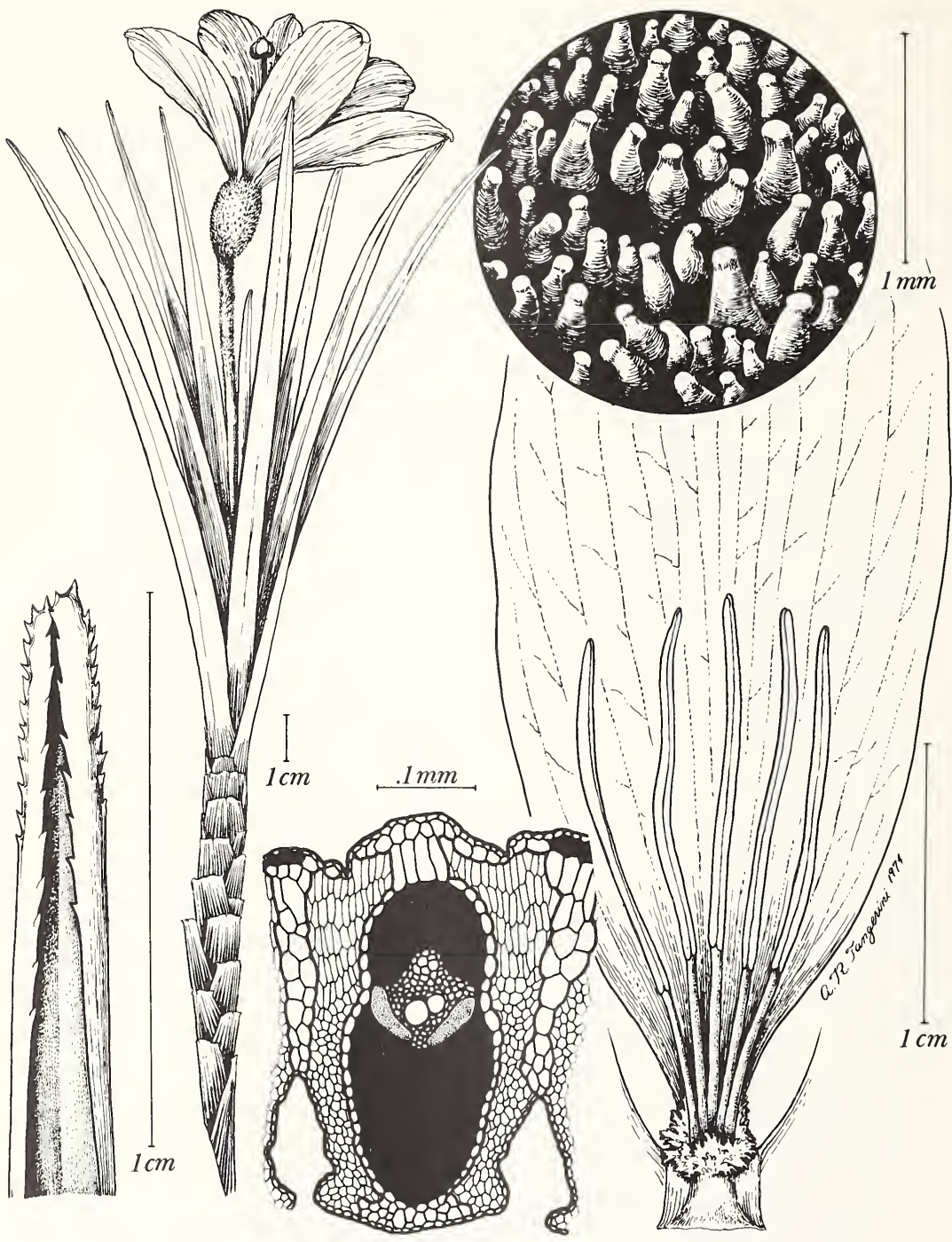

Figure 42.-Vellozia lilacina L. B. Smith \& Ayensu, new species (Menezes 238). 


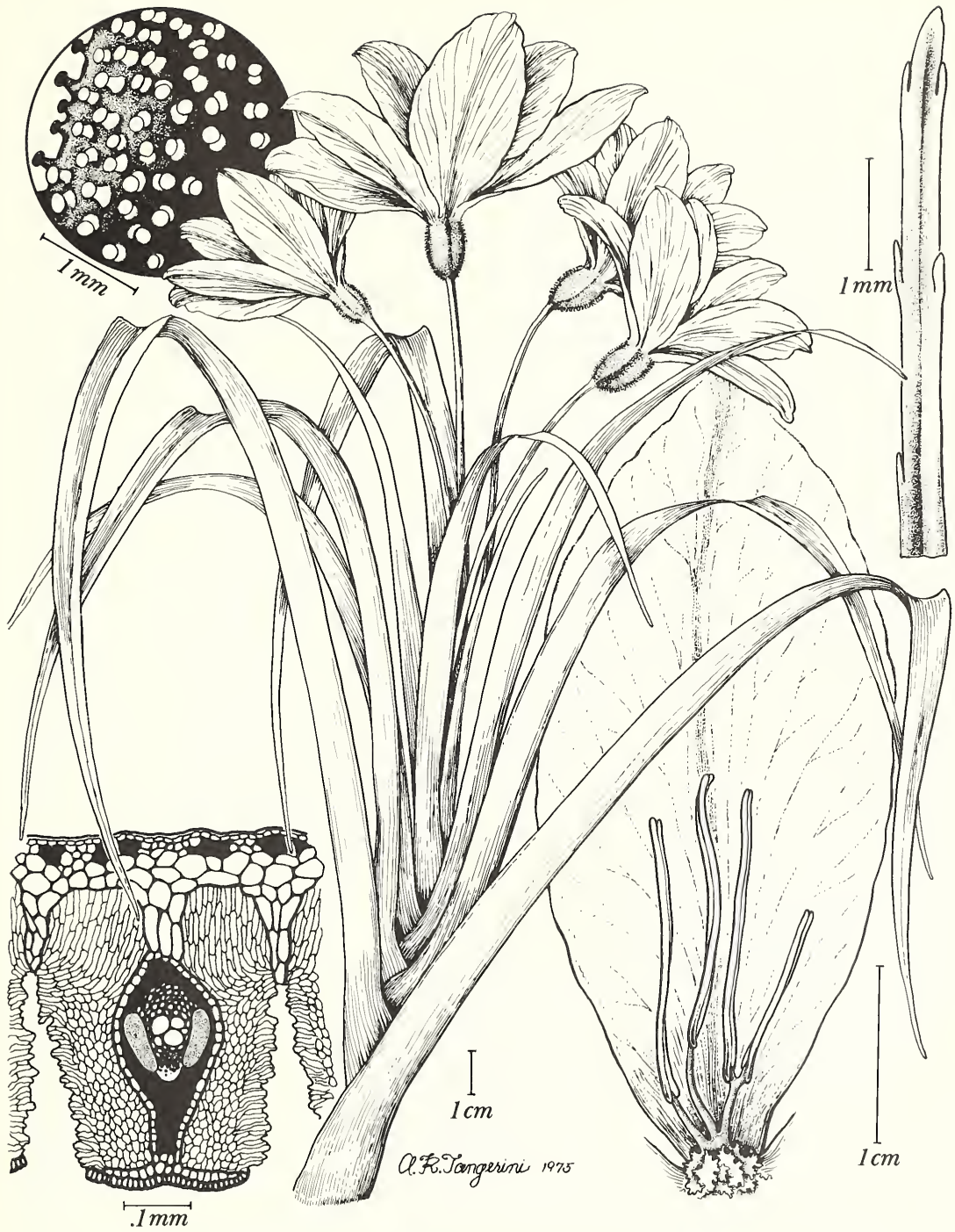

Figure 43.-Vellozia stipitata L. B. Smith \& Ayensu, new species (Semir \& Menezes 284). 
slightly ridged; abaxial surface furrowed $1 / 3$ to $2 / 3$ thickness of blade. Epidermis: cells on both surfaces rounded to dome-shaped; few conical, especially over midvein; thin-walled. Some adaxial epidermis replaced by sclerenchyma fibers in Menezes 230. Subjacent to adaxial epidermis is 1 or 2 layers of sclerenchyma fiber bundles interspersed with thin-walled cells. Below this is a layer of large, thin-walled parenchyma. Abaxial epidermis replaced by sclerenchyma fibers in Menezes 230 and Semir \& Menezes 284. Subjacent to abaxial epidermis in all sections, is a layer of thin-walled parenchyma cells. Cuticle: thin and smooth on abaxial surface; slightly thicker and ridged on adaxial. Stomata: present in abaxial furrows only; stomata flush with epidermal surface and have a small substomatal chamber. Stomata associated with large projections from walls of furrows. Mesophyll: 2-5 layers of palisade cells followed by compactly arranged, spongy tissue. One to three layers of large, translucent cells radially arranged above vascular bundles and furrows; large amount of cells above midvein. Sclerenchyma fibers usually present in abaxial corners of furrows. Vascular bundles: 21-35 (no count available for Semir \& Menezes 284); commissural bundles observed. One to three vessels present in each vascular bundle. Two phloem units lying laterally in flanges of Y-shaped abaxial girder. Adaxial cap present on each bundle. Bundle sheath completely surrounding each bundle. Crystals: none observed. Tannins: present.

Type.-Km 126 of the road from Lagoa Santa to Conceição do Mato Dentro, Serra do Cipó, Minas Gerais, Brazil, 3 November 1972, Semir \& Menezes 284 (SPF, holotype; US, photo).

Distribution.-Known only from the type-area.

\section{Vellozia decidua L. B. Smith \& Ayensu, new species}

Figure 44

A V. echinata Goethart \& Henrard, cui affinis, foliorum vaginis multo expositis, laminis latioribus, ovarii glandulis subsessilibus differt.

Caudex to $3 \mathrm{dm}$ high (IIrwin), $15 \mathrm{~mm}$ in diameter (including leaf-sheaths). Leaves many-ranked; sheaths subdensely imbricate and much exposed apically, splitting and curling with age; blades erect, deciduous, linear, attenuate, to $30 \mathrm{~cm}$ long,
$10 \mathrm{~mm}$ wide, flat, laxly serrulate, otherwise even and glabrous.

Scapes terminal, 3, more or less decurved in fruit, $7 \mathrm{~cm}$ long (above the leaf-sheaths), subdensely vestite with fine pale subsessile glands. Ovary (capsule) ellipsoid, $2 \mathrm{~cm}$ long, covered with pale subsessile glands; epigynous tube short. Stamens and style lacking.

Leaf ANatomy (Irwin et al. 22915).-Surface View: Hairs: few tufts present on both surfaces. Epidermis: cells square to rectangular on both surfaces; thin-walled. Stomata: paracytic, some tetracytic, $24 \times 15 \mu \mathrm{m}$; present in rows on adaxial surface and in abaxial furrows; few on abaxial surface.

Transverse Section of Lamina: Dorsiventral; Vshaped with median adaxial groove and 2 small grooves lateral to this. Adaxial surface undulating but ridged in places; abaxial furrowed $1 / 4$ thickness of blade; no furrows among first four bundles immediately lateral to midvein. Few tufts of hairs present on both surfaces. Epidermis: cells on both surfaces rounded to dome-shaped; few conical; thinwalled. Subjacent to adaxial epidermis is a layer of thin-walled parenchyma cells; few places have sclerenchyma fiber bundles. Subjacent to abaxial epidermis is a layer of thin-walled parenchyma cells. Cuticle: thin and smooth on adaxial surface; slightly thicker and ridged on abaxial surface. Stomata: present on adaxial and abaxial surfaces and in abaxial furrows; stomata flush with epidermal surface; small substomatal chamber present. Mesophyll: 3 or 4 layers of palisade cells followed by compactly arranged spongy tissue. Two to four layers of translucent mesophyll radially arranged above vascular bundles, furrows, and midvein. Sclerenchyma fiber bundles present at abaxial corners of furrows. Vascular bundles: 17; commissural bundles observed. One or two large vessels present in each vascular bundle, mostly one. Two phloem units lying laterally in flanges of thick, U-shaped abaxial girder. Adaxial cap present on each bundle. Bundles completely surrounded by a bundle sheath. Crystals: none observed. Tannins: few present.

Note: The shape of this species is very distinctive in that part of the lamina is furrowed and the free ends of the $\mathrm{V}$ are significantly enlarged.

TYPE.-Outcrops in cerrado and low gallery forest, ca. $25 \mathrm{~km}$ northeast of Diamantina on road to Mendanha, Minas Gerais, Brazil, $1200 \mathrm{~m}$ alt., 30 


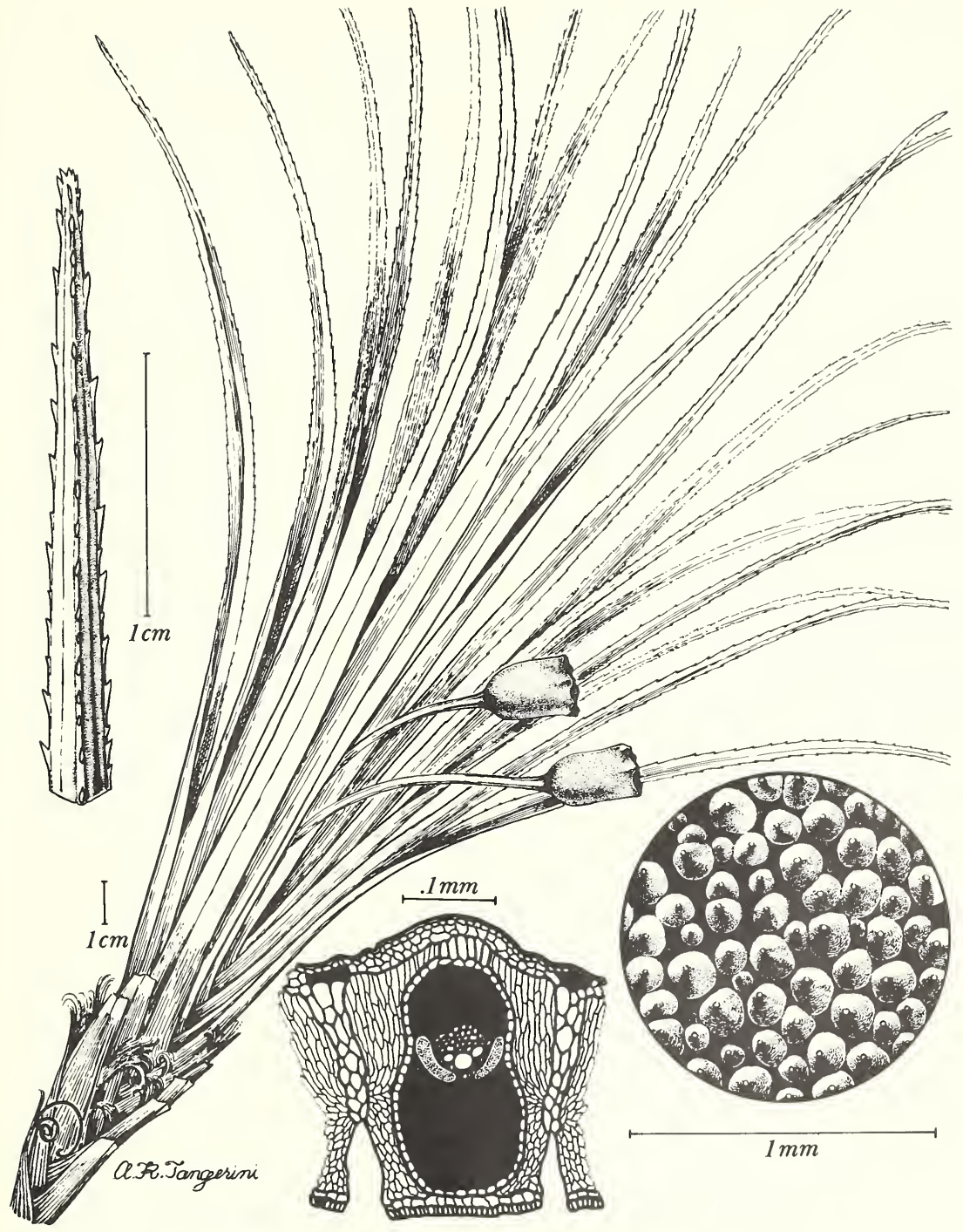

Frgure 44.-Vellozia decidua L. B. Smith \& Ayensu, new species (Irwin et al. 22915). 

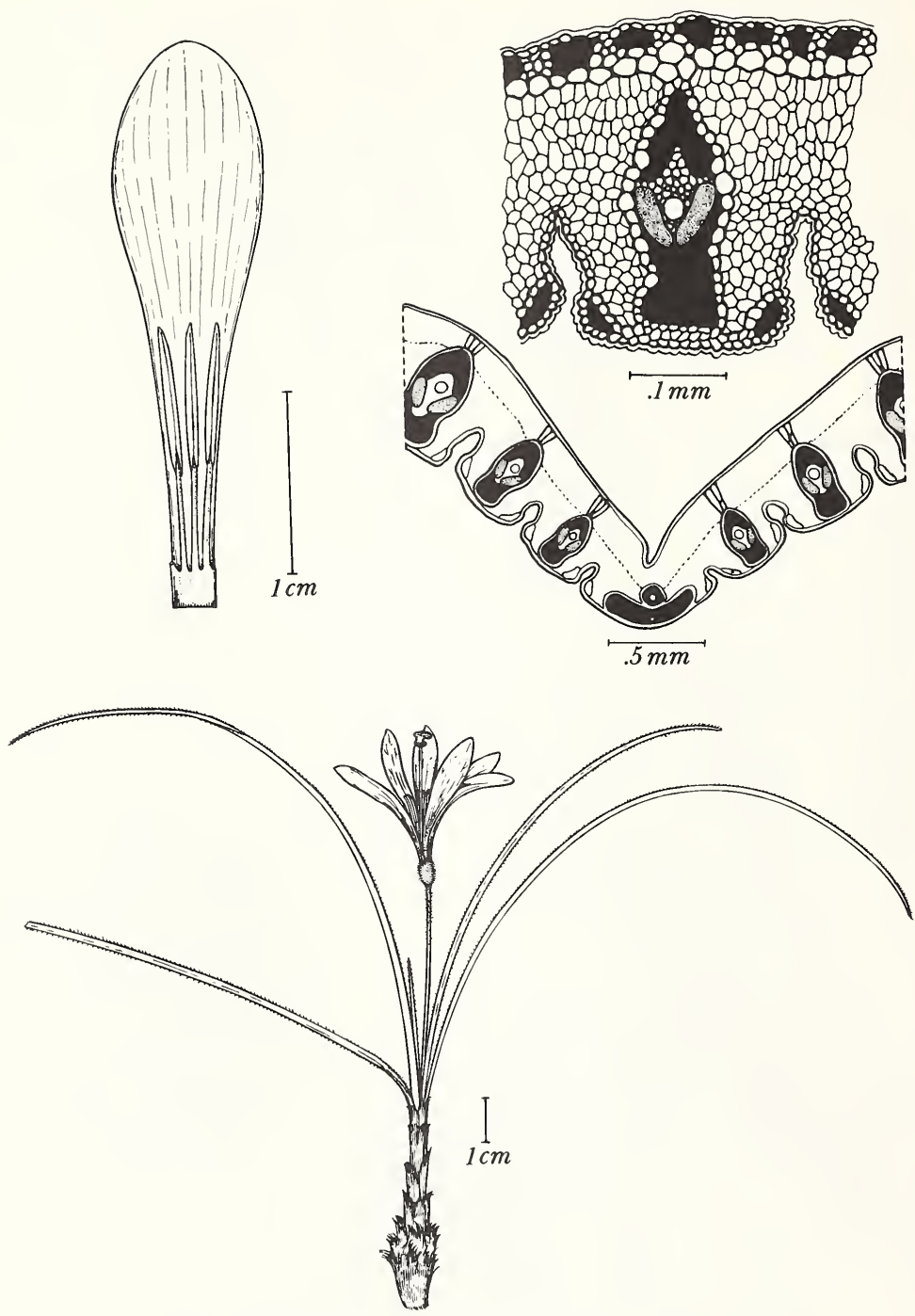

Figure 45.-Vellozia nanuzae L. B. Smith \& Ayensu, new species (Menezes 10). 
January 1969, Irwin et al. 22915 (US, holotype; NY, isotype).

Distribution.-Known only from the type-collection.

\section{Vellozia echinata Goethart \& Henrard}

Vellozia echinata Goethart \& Henrard, Blumea, 2:369, 1937.L. B. Smith, Contr. U.S. Nat. Herb., 35:261, 1962.-Ayensu, Smithsonian Contr. Bot., 15:21, pl. 36e, 1974.

TyPe.-Among rocks, Caraça, Minas Gerais, Brazil, 18 February 1884, Glaziou 15504 (L, holotype; $\mathrm{B}$, photo $15, \mathrm{~s} \mathrm{n}$; $\mathrm{P}$, isotype).

Distribution.--Known from the type-locality and from other collections without any locality.

\section{Vellozia nanuzae L. B. Smith \& Ayensu, new species}

\section{Figure 45}

A $V$. echinata Goethart \& Henrard, cui affinis, foliorum laminis angustissimis margine atroglandulosis solum ad apicem versus serrulatis differt.

Caudex to $8 \mathrm{dm}$ high, much branched, apically ca. $4 \mathrm{~mm}$ in diameter (including leaf-sheaths). Leaves 3-ranked, strongly odoriferous (!Menezes); sheaths subdensely imbricate, exposed apically for $1 \mathrm{~cm}$, soon splitting into fibers; blades early deciduous, linear, narrowly acute, $10-15 \mathrm{~cm}$ long (above the leaf-sheaths), $2 \mathrm{~mm}$ wide, revolute, the margin with dark sessile glands, serrulate only toward apex, otherwise even and glabrous.

Scapes 1 or 2, soon becoming lateral, 4-5 (-7) cm long (above the leaf-sheaths), finely pale-stipitateglandular. Ovary narrowly ellipsoid, 5-7 mm long, covered with slenderly stipitate pale glands; epigynous tube short. Tepals subspatulate, broadly rounded, $27 \mathrm{~mm}$ long, violet (!Menezes). Stamens ca. 18, ca. $13 \mathrm{~mm}$ long, phalanges unappendaged. Style much exceeding the stamens; stigmas terminal, orbicular, spreading.

LeAf АNAтомy (Menezes 10).-Surface View: None.

Transverse Section of Lamina: Dorsiventral; widely V-shaped. Adaxial surface slightly undulating; abaxial surface furrowed $1 / 3$ to $1 / 2$ thickness of leaf. Epidermis: cells on both surfaces rounded to dome-shaped; few conical; thin-walled. Adaxial cells interspersed with sclerenchyma fibers. Sub- jacent to adaxial epidermis are 2 layers rounded, thin-walled parenchyma cells interspersed with sclerenchyma fibers; below this is a layer of large, rounded, thin-walled cells. One layer of parenchyma subjacent to abaxial epidermis. Cuticle: thickened on both surfaces; relatively smooth on adaxial; very ridged on abaxial. Stomata: present in abaxial furrows; raised above epidermal surface; small substomatal chamber present. Mesophyll: 2 or 3 layers palisade cells followed by compact spongy tissue; cells translucent above midvein. Sclerenchyma fiber bundles present at corners of abaxial furrows. Vascular bundles; 13; commissural bundles not observed. One or two large vessels present in each bundle, mostly one. Two phloem units present in flanges of $\mathrm{Y}$-shaped abaxial girder. Adaxial cap present on each bundle. Each bundle completely surrounded by a bundle sheath. Crystals: observed in bundle sheath, especially on abaxial side. Tannins: few present.

Trpe.-Serra do Cipó, Minas Gerais, Brazil, 10 November 1968, Menezes 10 (US, holotype; SPF, isotype).

Distribution.-Brazil: Minas Gerais: Serra do Cipó, 19 October 1973, Magalhães Alves s $n$ (US). $\mathrm{Km} 127$ on right side of route from Lagoa Santa to Conceição do Mato Dentro, 8 October 1971, Menezes 157 (SPF, US). Same, km 114, 9 October 1971, Menezes 168 (SPF, US).

\section{Vellozia geotegens L. B. Smith \& Ayensu, new species}

\section{Figure 46}

A $V$. candida Mikan, cui verisimiliter affinis, caudice brevi, foliis densissime imbricatis, eorum laminis margine glandulosis haud serrulatis, tepalis violaceis differt.

Caudex very short, mat-forming (!Irwin). Leaves few in the terminal fascicle, glutinous at base (!Irwin); sheaths very densely imbricate with almost no apical exposure, dark castaneous, soon dividing into recurving fibers; blades becoming reflexed, persistent, very narrowly triangular, filiform-attenuate, to $32 \mathrm{~cm}$ long (above the leaf-sheaths), $8 \mathrm{~mm}$ wide at base, green with a dark castaneous spot at base.

Scapes terminal, 2 (or the caudex with very short branches), to $22 \mathrm{~cm}$ (flowering) or $30 \mathrm{~cm}$ long (fruiting), sulcate, glabrous. Ovary slenderly ellip- 
116

SMITHSONIAN CONTRIBUTIONS TO BOTANY

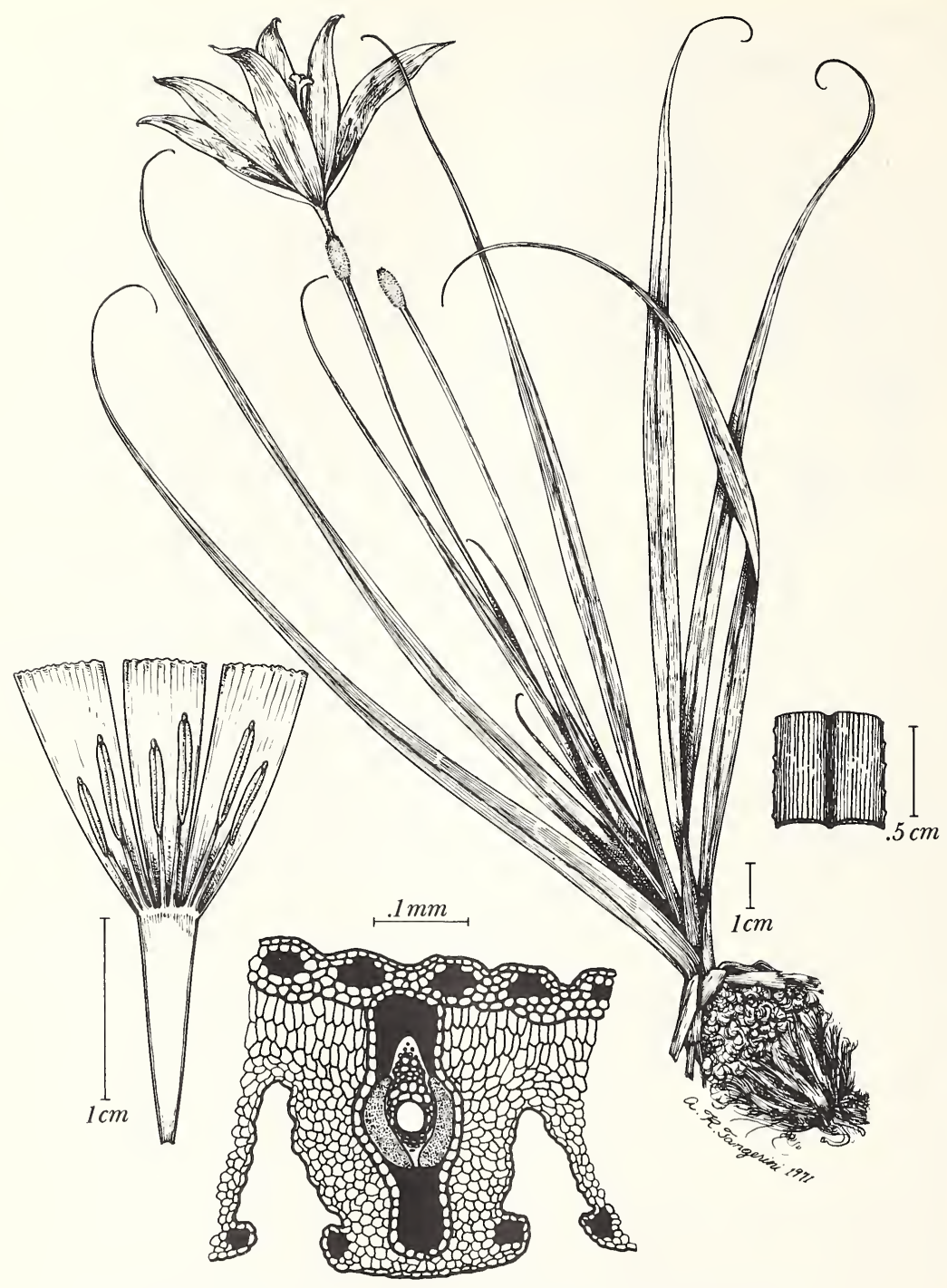

FigURE 46.-Vellozia geotegens L. B. Smith \& Ayensu, new species (Irwin et al. 22534). 
soid, $10 \mathrm{~mm}$ long, covered with fine tumid-stipitate glands; perianth tube short, deciduous. Tepals narrowly elliptic, $5 \mathrm{~cm}$ long including the $10 \mathrm{~mm}$ partially adherent claws, violet (!Irwin). Stamens 12 , largely adnate to the tepal-claws; anthers linear, $3 \mathrm{~mm}$ long, sagittate at base. Style exceeding the stamens; stigmas terminal, orbicular, spreading.

LeAF ANATomy (Irwin et al. 22534).-Surface View: Hairs: absent. Epidermis: adaxial and abaxial cells square to rectangular; thin-walled. Stomata: mostly paracytic, some tetracytic, $24 \times 15 \mu \mathrm{m}$; present only on abaxial surface.

Transverse Section of Lamina: Dorsiventral; V-shaped. Adaxial surface slightly undulating; abaxial surface furrowed about $1 / 2$ thickness of blade. Epidermis: cells on adaxial and abaxial surfaces are rounded and thin-walled; larger above the midvein. Subjacent to adaxial epidermis are 2 or 3 layers of rounded cells interspersed with fiber bundles; fiber strands also present at corners of furrows subjacent to abaxial epidermis. Cuticle: uniformly thickened on entire surface; thinner in median area of adaxial surface. Stomata: present in abaxial furrows only; slightly extending above epidermal surface; substomatal chamber not observed. Mesophyll: 3 or 4 layers of rectangular, palisadelike cells grading into compactly arranged spongy tissue. Vascular bundle: 25; few commissural bundles observed. Usually one large vessel in each bundle; rarely two. Two phloem units lying laterally in flanges of $Y$-shaped abaxial girder. Each bundle accompained by an adaxial cap. Bundle sheath completely surrounding each bundle. Crystals and tannins: none observed.

Trpe.-Outcrops by campo, near Datas, Minas Gerais, Brazil, $1300 \mathrm{~m}$ alt, 24 January 1969, Irwin et al. 22534 (US, holotype; NY, isotype).

Distrirution.-Known only from the typecollection.

\section{Vellozia candida Mikan}

Vellozia candida Mikan, Delect. Fl. \& Faun. Brazil, 2, pl. 1, 1820; text, 1825.-Sprengel, Syst. Veg., 3:338, 1826.-Seubert in Martius, Fl. Bras., 3(1):82, 1847. Hooker f., Bot. Mag., 91, pl. 5514, 1865.-L. B. Smith, Contr. U.S. Nat. Herb., 35:267, 1962.-Ayensu, Smithsonian Contr. Bot., 15:46, fig. 15a-c, pls. 18a, 34g-h, 35a, 50b, 1974.

Vellozia tertia Sprengel, Neu. Entd., 2:108, 1821 [type: no collection cited; evidently the species name refers to the third species known to Mikan and is thus invalid as an unnecessary renaming of a valid species].
Vellozia maritima Vellozo, Fl. Flum., 219, 1825.-Icon., 5, pl. 79, 1831 [type: Rocks, Island of Parati, Rio de Janeiro, Brazil; none extant now if there ever were any; recent collections from Parati show no difference from highmontane specimens].

TYPE.-Colonial on summit of the Corcovado and on rocks toward Boa Vista, city of Rio de Janeiro, Brazil. Mikan s $n$ (W, holotype, lost).

Distribution.-Brazil: Rio de Janeiro: Paratí, Rio de Janeiro.

\section{Vellozia aloifolia Martius}

Vellozia aloifolia Martius, Nov. Gen. \& Sp., 1:15, pl. 7, 1823.Seubert in Martius, F1. Bras., 3(1):78, 1847.-L. B. Smith, Contr. U.S. Nat. Herb., 35:261, 1962.-Ayensu, Smithsonian Contr. Bot., 15:12, fig. 10a-c, pl. 29d-f, 1974.

TYPE.-Rocky slope, Serra de Itambé da Villa do Principe (Sêrro), $1200 \mathrm{~m}$ alt, Minas Gerais, Brazil, June 1818, Martius 1363 (M, holotype; US, photo).

Distribution.-Brazil: Minas Gerais: Diamantina, Sêrro.

\section{Vellozia metzgerae $\mathbf{L}$. B. Smith}

Vellozia metzgerae L. B. Smith, Phytologia, 8:512, figs. 10, 11, 1963.

Type.-Serra da Lapa (Cipó), Minas Gerais, Brazil, November 1824, Riedel s $n$ (K, holotype).

Distribution.-Known only from the typecollection.

100. Vellozia caespitosa L. B. Smith \& Ayensu, new species

Figure 47

A $V$. glandulifera Goethart \& Henrard, cui affinis, foliorum laminis subtus laxe tomentosis nervis haud occultis, tepalis valde majoribus differt.

Caudex very short but branching and forming mats, apically $15 \mathrm{~mm}$ in diameter (including leafsheaths). Leaves about 10 in the terminal fascicle; sheaths subdensely imbricate exposing ca. $5 \mathrm{~mm}$ apically, dark castaneous, finely appressedtomentose, soon dissolving into coarse fibers; blades erect, soon deciduous, linear, acute, $16 \mathrm{~cm}$ long (above the leaf-sheaths), $10 \mathrm{~mm}$ wide, bicarinate and obscurely serrulate toward apex, revolute, gla- 


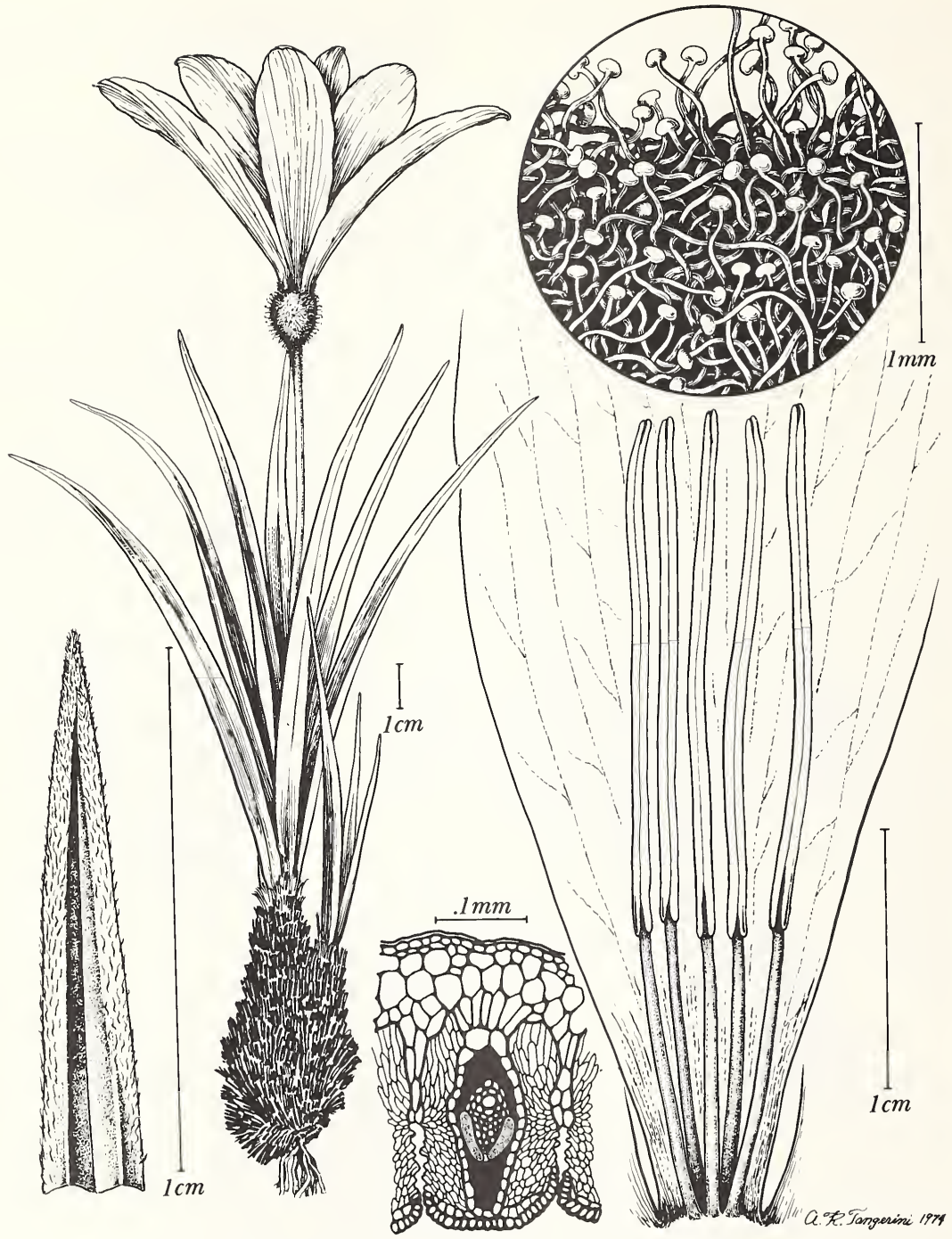

F1Gure 47.-Vellozia caespitosa L. B. Smith \& Ayensu, new species (Irwin et al. 22373). 
brous above, sparsely tomentose beneath leaving the nerves clearly visible.

Scapes terminal, solitary $12-16 \mathrm{~cm}$ long (above the leaf-sheaths), ca $2 \mathrm{~mm}$ in diameter, densely and finely stipitate-glandular. Ovary turbinate, $10 \mathrm{~mm}$ long, covered with small glands on long fine contorted pale stipes. Tepals elliptic, to $60 \mathrm{~mm}$ long, dark violet. Stamens ca. 18, $3 \mathrm{~cm}$ long; phalanges unappendaged. Style much exceeding stamens; stigmas terminal, orbicular, spreading.

LeAF ANATOMy (Irwin et al. 22373).-Surface View: Hairs: few small tufts present on adaxial; long, single hairs present on abaxial surface. Epidermis: cells square to rectangular on both surfaces; thin-walled. Stomata: paracytic, $21 \times 15 \mu \mathrm{m}$; present in abaxial furrows only.

Transverse Section of Lamina: Dorsiventral; deeply V-shaped with median adaxial groove and margins curved downward. Adaxial surface undulating; abaxial surface furrowed about $1 / 4$ thickness of blade. Hairs present on abaxial surface, and on extreme margins of adaxial surface. Epidermis: cells on both surfaces rounded to dome-shaped; thin-walled. Subjacent to adaxial epidermis is 2 or 3 layers of rounded, thin-walled parenchyma cells. There is a single layer of distinct parenchyma cells subjacent to abaxial epidermis. Cuticle: thick and smooth on adaxial surface; thin and smooth on abaxial surface. Stomata: present in abaxial furrows only; stomata flush with epidermal surface and associated with many small papillae in furrow; small substomatal chamber present. Mesophyll: 3 or 4 layers of palisade cells followed by compactly arranged rounded spongy cells. Palisade cells above vascular bundles and furrows often large and translucent. Vascular bundles: 31; commissural bundles observed. One or two large vessels present in each bundle, mainly one. Two phloem units lying laterally in flanges of $\mathrm{Y}$-shaped abaxial girder. Fairly large adaxial cap present on each bundle. Each bundle surrounded by a bundle sheath. Crystals: none observed. Tannins: few present.

TYPE--On rocks, cerrado on steep rocky slopes, ca. $20 \mathrm{~km}$ southwest of Diamantina, Minas Gerais, Brazil, $1300 \mathrm{~m}$ alt, 21 January 1969, Irwin et al. 22373 (US, holotype; NY, isotype).

Distribution.-Known only from the typecollection.

\section{Vellozia glandulifera Goethart \& Henrard}

Vellozia glandulifera Goethart \& Henrard, Blumea, 2:372, 1937.-Ayensu, Smithsonian Contr. Bot., 15:24, fig. 2c, pls. lc,d, 36f, 1974.

Vellozia bicolor L. B. Smith, Contr. U.S. Nat. Herb., 35:287, pl. 11: figs. 43-45, 1962.-Ayensu, Smithsonian Contr. Bot., 15:13, fig. 2a, pl. 42a, 1974 [type: on rocky sandy soil, summit of Serra do Cipó, road from Hotel Chapeu do Sol between $\mathrm{km} 111$ and 120, Minas Gerais, Brazil, $1200 \mathrm{~m}$ alt., 6 August 1960, Maguire, Magalhães or Maguire 49037 (NY, US)].

Vellozia mollis L. B. Smith, Contr. U.S. Nat. Herb., 35:287, pl. 11: figs. 46-48, 1962 [type: on sandstone outcrop, ca. 3-5 $\mathrm{km}$ east of Serra, along road from Conceição to Diamantina, Minas Gerais, Brazil, 9 August 1960, Maguire, Magalhães o Maguire 49156 (NY, US)].

Vellozia vestita L. B. Smith, Phytologia, 8:511, figs. 8, 9, 1963 [type: without locality, Minas Gerais, Brazil, 1892, Glaziou 19935 (K, type)].

Type.-Cachoeira, [Minas Gerais], Brazil, Langsdorff in herb. Fischer 1418 (LE, holotype; K, isotype?).

Distribution.-Brazil: Minas Gerais: Cachoeira, Diamantina, Jaboticatubas, Sêrro.

\section{Section Radia (A. Richard) L. B. Smith \&} Ayensu, new combination, new status

Radia A. Richard in Kunth, Syn. Pl. Aequin., 1:300, 1822.

Leaf-blades more or less flattened; furrows almost always distinct on the abaxial side. Tepals with their claws fused into a long slender tube. Stamens more than 6.

TYPE-SPEC1Es.-Radia tubiflora A. Richard.

\section{Vellozia markgrafii Schulze-Menz}

Vellozia markgrafii Schulze-Menz in Markgraf, Notizblatt, 15:216, 1940.-L. B. Smith, Contr. U.S. Nat. Herb., 35:267, 1962.

TyPE.-On argyllite, Serra de São Calixto, 13 $\mathrm{km}$ northeast of Hotel Flamingo, east of Montes Claros, Minas Gerais, Brazil, $100 \mathrm{~m}$ alt, 11 November 1938, Markgraf, Brade \& Mello Barreto 3373 (B? $\mathrm{n} v$ ).

Distributron.-Brazil: Minas Gerais: on outcrop in cerrado and wet rocky campo, $8 \mathrm{~km}$ west of Grão Mogul, $950 \mathrm{~m}$ alt, 16 February 1969, Irwin et al. 23377 (NY, UB, US), det. ex char. 


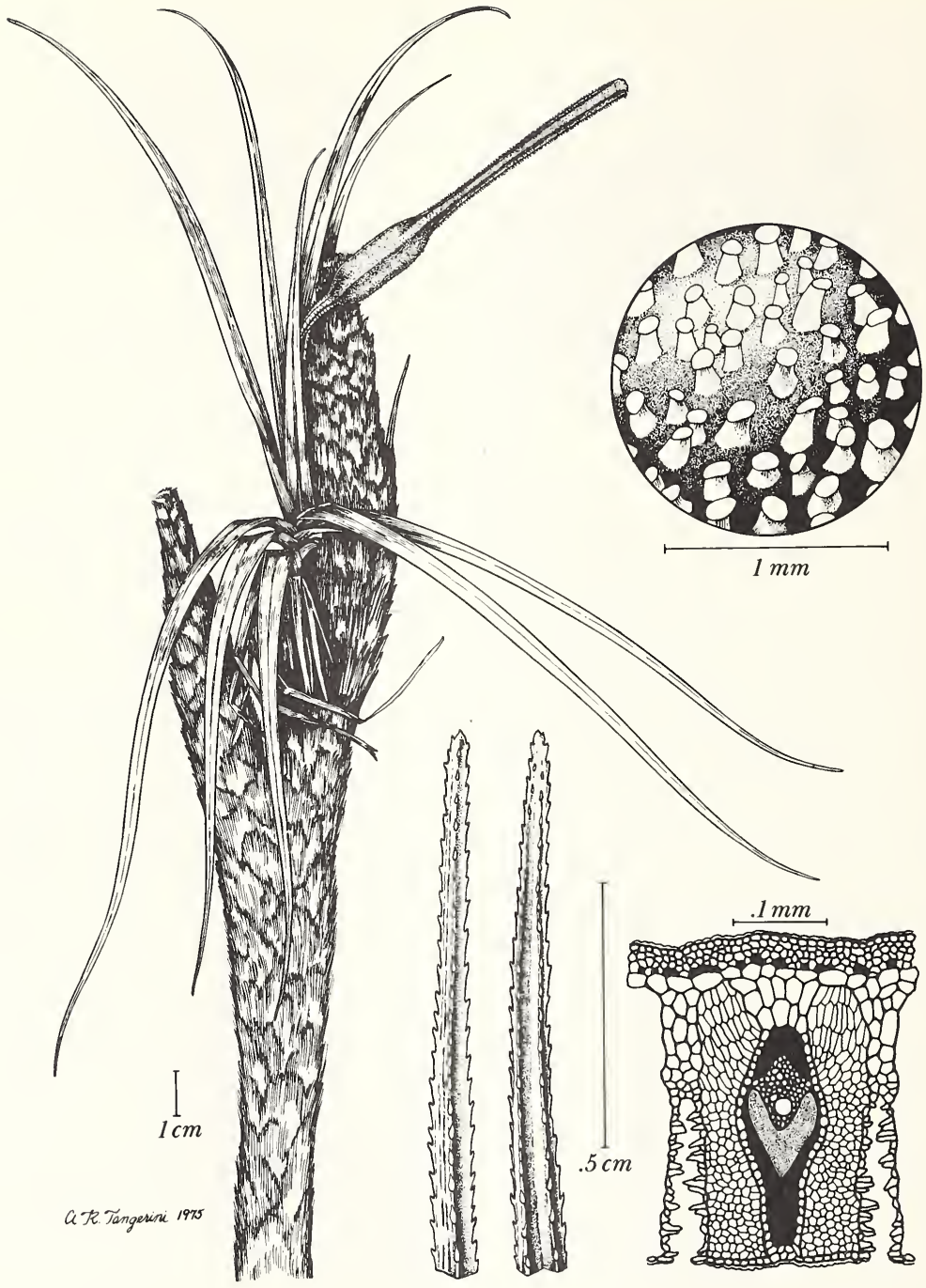

Figure 48.-Vellozia caput-ardeae L. B. Smith \& Ayensu, new species (L. B. Smith, Ayensu \& Hatschbach 15989). 
103. Vellozia caput-ardeae L. B. Smith \& Ayensu, new species

\section{FigURE 48}

$\mathrm{Ab}$ omnibus speciebus adhuc cognitis foliis supra glandulis pallidis confluentibus auctis, tubo perianthii elongato persistente differt.

Caudex erect, over $1 \mathrm{~m}$ high, branched, over 15 $\mathrm{mm}$ in diameter (including leaf-sheaths). Leaves few in the terminal fascicle, glutinous; sheaths subdensely imbricate and exposing only about $5 \mathrm{~mm}$ apically, erect and dividing into coarse fibers; blades becoming reflexed, persistent, very narrowly triangular, filiform-attenuate, to $21 \mathrm{~cm}$ long, $7 \mathrm{~mm}$ wide at base, ventrally at base bearing broad pale glands that are confluent above, the thick yellow margins closely serrulate.

Scapes terminal, solitary, curved, $8 \mathrm{~cm}$ long (above the leaf-sheaths), finely and subdensely stipitateglandular. Ovary ellipsoid, $25 \mathrm{~mm}$ long, finely and subdensely stipitate-glandular; perianth tube slenderly subcylindric, $7 \mathrm{~cm}$ long, persistent. Tepals and stamens unknown.

Leaf Anatomy.-Ayensu, Smithsonian Contr. Bot., 15:46, pl. 36a, 1974.

Type.-Rock dome, Guinda, Mun. Diamantina, Minas Gerais, Brazil, 19 January 1972, L. B. Smith, Ayensu \& Hatschbach 15989 (US, holotype).

Distribution.-Known only from the type-area.

\section{Vellozia maguirei L. B. Smith}

Vellozia maguirei L. B. Smith, Contr. U.S. Nat. Herb., 35:267, pl. 5,1962 .

Type.-Some $78 \mathrm{~km}$ (49 miles) from Diamantina, Minas Gerais, Brazil, $1140 \mathrm{~m}$ (3800 ft) alt, $22 \mathrm{De}-$ cember 1959, Maguire, Maguire \& Pires 44742 (US, holotype; NY, isotype).

Distribution.-Known only from the typecollection.

\section{Vellozia maudeana $\mathbf{R}$. E. Schultes}

Vellozia maudeana R. E. Schultes, Bot. Mus. Leafl. Harvard, 16:198, pls. 29, 30, 1954.-L. B. Smith, Contr. U.S. Nat. Herb., 35:269, 1962.

TyPE.-Quartzite savanna. Goo-rán-hoo-da, Mesa de Yambí, Río Karurú (tributary of Río Vaupés), Vaupés, Colombia, $1{ }^{\circ} 20^{\prime} \mathrm{N}, 71^{\circ} 20^{\prime} \mathrm{W}$, ca. $285 \mathrm{~m}$
(950 ft) alt, 15-16 April 1953, Schultes \& Cabrera 19120 (GH, holotype; US, isotype).

Distribution.-Known only from the typecollection.

106. Vellozia uleana L. B. Smith

FIGURE 49

Vellozia uleana L. B. Smith, Contr. U.S. Nat. Herb., 35:269, pl. 9: figs. 13, 14, 1962.

Leaf ANAтомy (Ule 8372).-Surface View: Hairs: few large tufts observed. Epidermis: cells on both surfaces square to rectangular; thin-walled. Stomata: tetracytic, $21 \times 12 \mu \mathrm{m}$; present in abaxial furrows only.

Transverse Section of Lamina: Dorsiventral; widely $\mathrm{V}$-shaped with small median adaxial groove and margins curved downward. Few tufts of hairs observed on both surfaces. Adaxial surface undulating; abaxial surface furrowed $1 / 2$ thickness of blade. Epidermis: adaxial cells square to rectangular; few crescent-shaped; thin-walled. Abaxial cells rounded to dome-shaped; thin-walled. Subjacent to adaxial epidermis is 1 or 2 layers of thin-walled parenchyma interspersed with 1 or 2 layers of sclerenchyma fiber bundles. Below this is a layer of large, thin-walled parenchyma cells. Subjacent to abaxial epidermis is a layer of thin-walled parenchyma cells. Cuticle: thick and ridged on both surfaces. Stomata: present in abaxial furrows only; flush with epidermal surface. Mesophyll: 3 or 4 layers of palisade cells followed by compactly arranged spongy mesophyll. Two or three layers of translucent cells radially arranged above vascular bundles, furrows, and midvein. One or two layers of sclerenchyma fiber bundles present at abaxial corners of furrows; partially replacing some abaxial epidermal cells. Vascular bundles: 27; commissural bundles not observed. One or two large vessels present in each bundle, mostly one. Two phloem units lying laterally in flanges of $\mathrm{Y}$-shaped abaxial girder. Adaxial cap present on each bundle. Bundle sheath completely surrounding each vascular bundle. Crystals: none observed. Tannins: present.

TyPE.-Rock faces of Serra de Uairary (?Mairarí), Surumú, Rio Branco, Brazil, 1000-1200 m alt, September 1909, Ule 8372 (MG, holotype; L; NY; US, isotypes). 


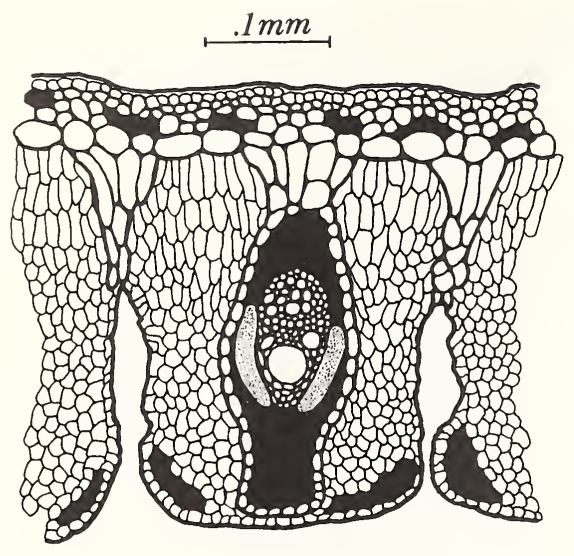

Figure 49.-Vellozia uleana L. B. Smith (Ule 8372).

Distribution.-Known only from the typecollection.

107. Vellozia maculata Goethart \& Henrard Figure 50

Vellozia maculata Goethart \& Henrard, Blumea, 2:375, 1937. L. B. Smith, Contr. U.S. Nat. Herb., 35:268, 1962.-Ayensu, Smithsonian Contr. Bot., 15:50, pls. 3c-d, 45f, 1974.

TYPE.-Cachoeiras da Vargem Grande da Serra da Balisa [Goiás], Brazil, 5 January 1895, Glaziou 22218-a (P, holotype; L, isotype; $\mathbf{B}$, photo 87,90 ).

Distribution.-Known only from the typecollection.

\section{Vellozia machrisiana L. B. Smith}

Vellozia machrisiana L. B. Smith, Contr. U.S. Nat. Herb., 35:268, pl. 9: figs. 11, 12, 1962.-Ayensu, Smithsonian Contr. Bot., 15:50, fig. 17d-f, pls. 4a--b, 35b-d, 1974.

TYPE.-On rocks in stream below falls (subject to running water), sandstone area $14 \mathrm{~km}$ south of Veadeiros, region of the Chapada dos Veadeiros,

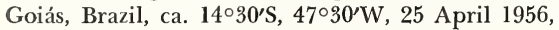
Dawson 14674 (US, holotype).

Distribution.-Known only from the typecollection.

\section{Vellozia lanata Pohl}

\section{Figure 51}

Vellozia lanata Pohl, Pl. Bras., 1:130, 1827.-Seubert in Martius, Fl. Bras., 3(1)83, 1847.-L. B. Smith, Contr. U.S. Nat. Herb., 35:284, 1962.-Ayensu, Smithsonian Contr. Bot., 15:49, pls. 3a-b, 17b, 45e, 1974.

Vellozia cana Goethart \& Henrard, Blumea, 2:367, 1937.Ayensu, Smithsonian Contr. Bot., 15:46, pl. 45b, 1974 [type: among rocks, Paranana, Goiás, Brazil, 12 February 1895, Glaziou 22219 (L, holotype; B, photo 92, s n)].

TYPE.-Dry rocky mountains, Caldas Novas, Goiás, Brazil, ca. $16^{\circ} 48^{\prime} \mathrm{S}$, Pohl s $n$ (M, holotype; F, photo 18978).

Distribution.-Brazil: Maranhão: Serra da Melicia, near Carolina, Rio Tocantins, 27 May 1950, Pires \& Black 2351 (IAN, US, sterile, identification not certain). Minas Gerais: Patrocinio. Goiás: Alto Paraizo (Chapada dos Veadeiros), Goiás Velho (Serra Dourada), Pirenópolis, Veadeiros. Distrito Federal: Gama.

\section{Vellozia hirsuta Goethart \& Henrard}

Vellozia hirsuta Goethart \& Henrard, Blumea, 2:374, 1937.-

L. B. Smith, Contr. U.S. Nat. Herb., 35:284, 1962.-Ayensu, Smithsonian Contr. Bot., 15:49, fig. 4d, pl. 37b, 1974.

TYPE.-Biri-biri, near Diamantina, Minas Gerais, Brazil, 24 March 1892, Glaziou 19924 (L, holotype; $\mathrm{B}$, photo), leaves only.

Distribution.-Brazil: Minas Gerais: rock outcrops, Mun. Gouvêa, Serra do Espinhaço, 16 November 1971, Hatschbach 28093 (MBM, US). Rock outcrops, Mun. Diamantina, $21 \mathrm{~km}$ north of Diamantina, 20 January 1972, L. B. Smith, Ayensu b Hatschbach (separate no. 28996) 15999 (MBM, US).

Note: The very nearly topotypic collections above show flowers typical of Section Radia and enable inclusion of the species in our key.

\section{Vellozia dawsonii L. B. Smith}

Vellozia dawsonii L. B. Smith, Contr. U.S. Nat. Herb., 35:270, pl. 7, 1962.-Ayensu, Smithsonian Contr. Herb., 15:48, fig. 3g-h, pls. la-b, 45c, 5la, 1974.

TYPE.-On sandstone outcrop, $7 \mathrm{~km}$ south of Veadeiros, region of the Chapada dos Veadeiros, Goiás, Brazil, ca. $14 \circ 30^{\prime} \mathrm{S}, 47^{\circ} 30^{\prime} \mathrm{W}, 24$ April 1956, Dawson 14580 (US, holotype).

Distribution.-Known only from the type-area. 


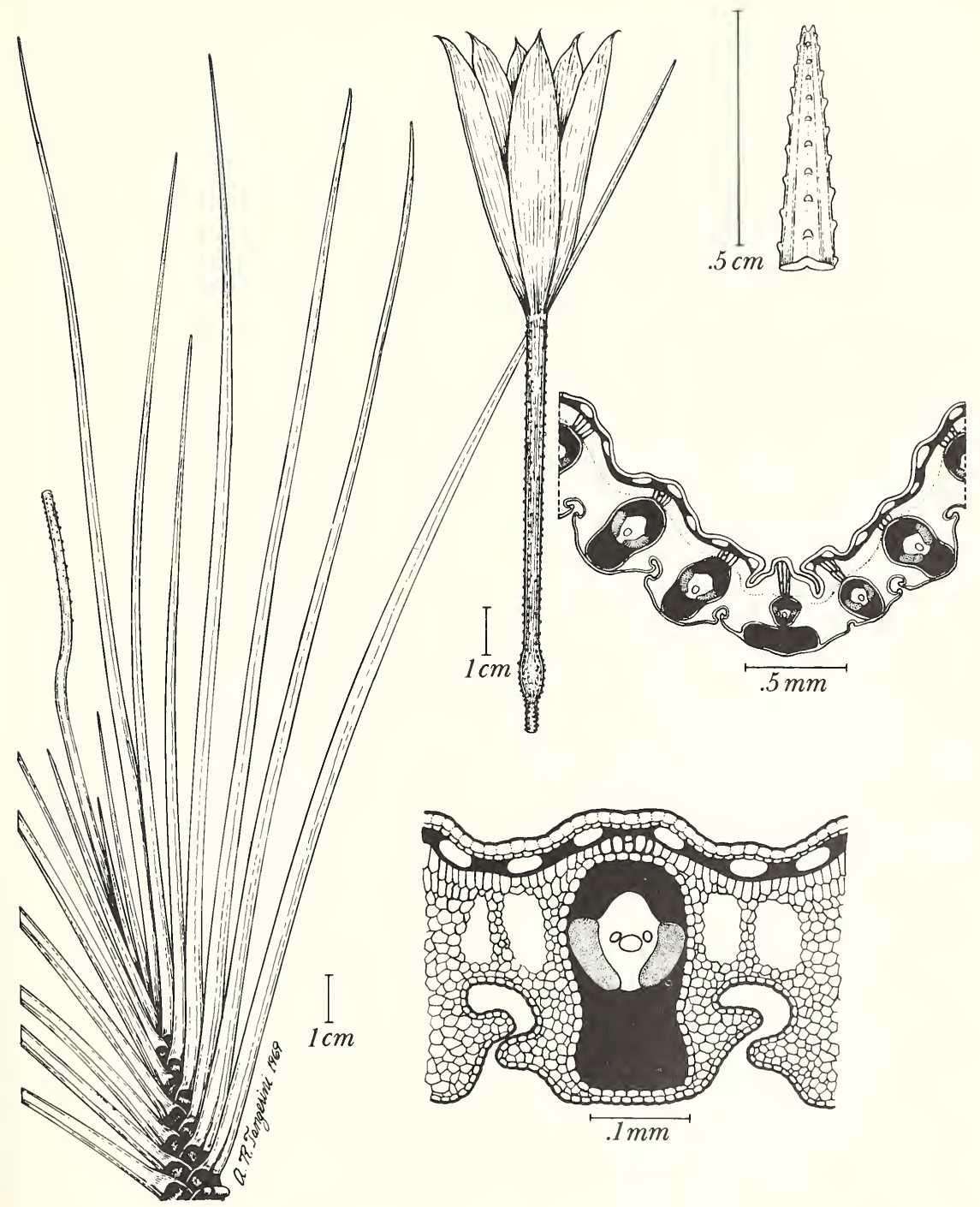

Figure 50.-Vellozia maculata Goethart \& Henrard (Glaziou 22218-a). 

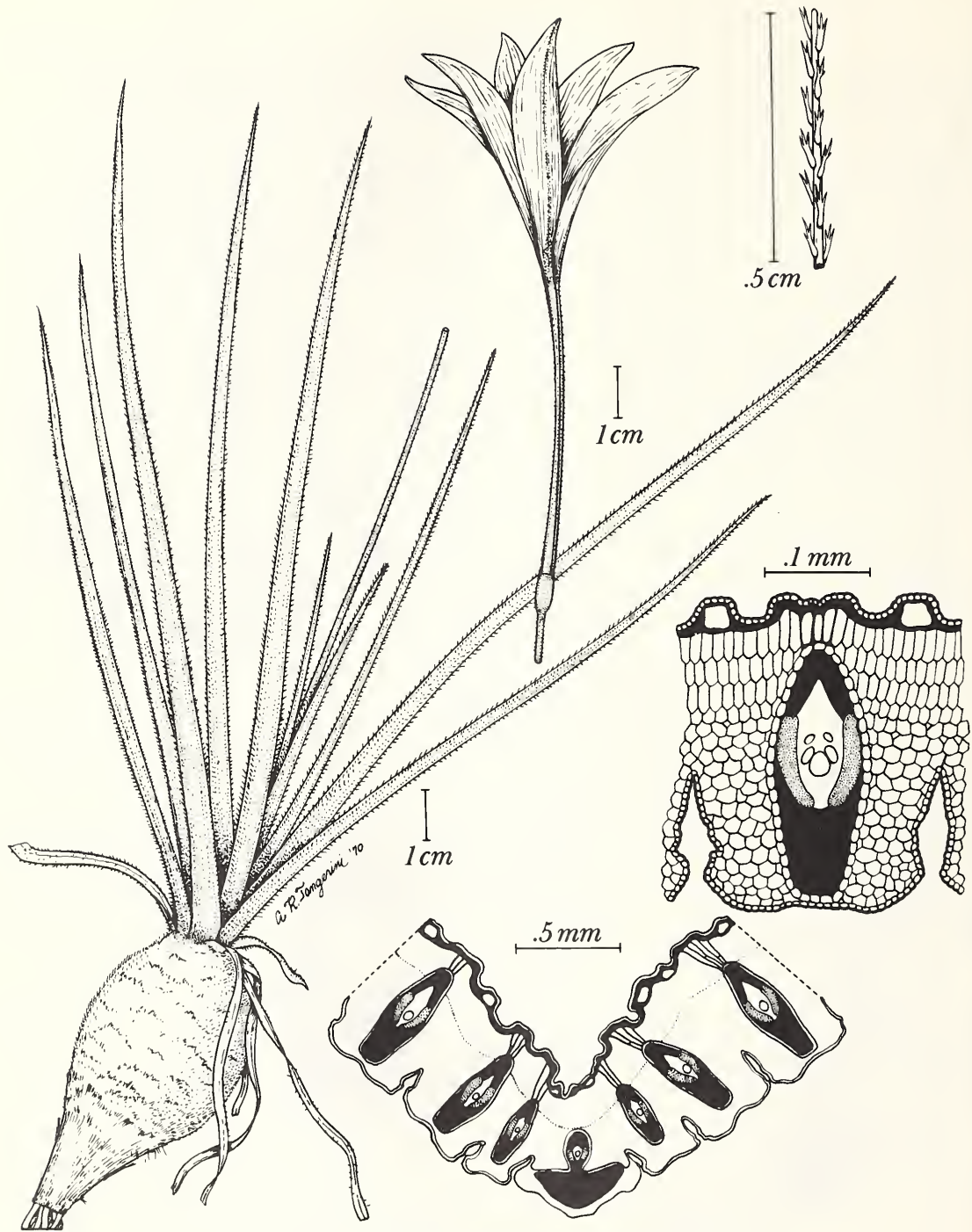

Figure 51.-Vellozia lanata Pohl (Vera Lúcia \& Graziela s $n$ ). 


\section{Vellozia velutinosa Goethart \& Henrard}

Vellozia velutinosa Goethart \& Henrard, Blumea, 2:382, 1937. -L. B. Smith, Contr. U.S. Nat. Herb., 35:269, 1962.

Type.-Cabeceira (headwaters) of Rio Santa Ana, Goiás, Brazil, 8 January 1895, Glaziou 22218 (L, holotype; B, photo 89).

Distribution.-Known only from the typecollection.

\section{Vellozia phantasmagorica $\mathbb{R}$. E. Schultes}

Vellozia phantasmagorica R. E. Schultes, Bot. Mus. Leaf. Harvard, 12:130, pls. 19, 20, 1946.-L. B. Smith, Contr. U.S. Nat. Herb., 35:269, 1962.

TYPE.-On sandstone, savanna, Mount Chiribiquete, Macaya River, upper Apaporis basin, Vaupés, Colombia, 390-630 $\mathrm{m}$ alt, 18 January 1944, R. E. Schultes 5741 (GH, holotype; COL, US, isotypes).

Distribution.-Colombia: Vaupés: Mount Chiribiquete. Meta: San José del Guaviare.

\section{Vellozia annulata Goethart \& Henrard}

\section{F1GURE 52}

Vellozia annulata Goethart \& Henrard, Blumea, 2:365, 1937. -L. B. Smith, Contr. U.S. Nat. Herb., 35:269, 1962.

Leaf Anatomy (Glaziou 22212).-Surface View: Hairs: tufts of hairs present on both surfaces. Epidermis: cells on both surfaces square to rectangular; thin-walled. Stomata: paracytic and tetracytic; $21 \times 18 \mu \mathrm{m}$; present on both surfaces and in abaxial furrows.

Transverse Section of Lamina: Dorsiventral; widely V-shaped. Adaxial surface ridged; abaxial surface furrowed about $1 / 2$ thickness of blade. Tufts of hairs present on both surfaces. Epidermis: cells on both surfaces rounded to dome-shaped; few conical; thin-walled. Few sclerenchyma fiber bundles present in layer subjacent to adaxial epidermis; almost a complete layer of sclerenchyma fibers in layer subjacent to abaxial epidermis. Cuticle: thick and ridged on entire surface. Stomata: present on both surfaces especially in abaxial furrows; stomata flush with epidermal surface; small substomatal chamber present. Mesophyll: 4 or 5 layers of palisade cells; many translucent, especially directly above vascular bundles and furrows; this is followed by 12 or 13 layers rounded, thin-walled, compactly arranged cells. Vascular bundles: 17; commissural bundles observed. One or two xylem vessels present in each bundle; mostly one. Two phloem units lying laterally in flanges of thick, Y-shaped abaxial girder. Adaxial cap present on each bundle. Bundle sheath completely surrounding each vascular bundle. Crystals: none observed. Tannins: present.

TYPE.-Among rocks, Cachoeira da Vargem Grande, Goiás, Brazil, 4 January 1895, Glaziou 22212 (L, holotype; B, photo 93, s n).

Distribution.-Brazil: Goiás: Cachoeira da Vargem Grande, Veadeiros.

\section{Vellozia leucanthos Goethart \& Henrard}

Vellozia leucanthos Goethart \& Henrard, Blumea, 2:375, 1937. -L. B. Smith, Contr. U.S. Nat. Herb., 35:268, 1962.

Vellozia cachimbensis L. B. Smith, Contr. U.S. Nat. Herb., 35:268, pl. 6, 1962.-Ayensu, Smithsonian Contr. Bot., 15: 45 , figs. $3 \mathrm{~m}-\mathrm{n}, 15 \mathrm{~d}-\mathrm{f}, 1974$ [type: on rocks, Serra do Cachimbo, Pará, Brazil, $425 \mathrm{~m}$ alt, 14 December 1956, Pires, Black, Wurdack \& Silva 6232 (IAN, holotype)].

TyPE.-In campo, Serra do Ificionado, near Caraça, Minas Gerais, Brazil, 10 July 1883, Glaziou 15674 (L, holotype; B, photo 88, s n).

Distribution.-Brazil: Pará: Serra do Cachimbo. Minas Gerais: Caraça.

\section{Vellozia panamensis Standley}

Vellozia panamensis Standley, Journ. Washington Acad. Sci., 15:457, 1925.-L. B. Smith, Contr. U.S. Nat. Herb., 35:269, 1962 .

TyPE.-In savannas, Cerro Vaca, eastern Chiriqui, Panama, 900-1136 m alt, 25-28 December 1911, H. Pittier 5352 (US, holotype).

Distribution.-Known only from the typecollection.

\section{Vellozia riedeliana Goethart \& Henrard}

Vellozia riedeliana Goethart \& Henrard, Blumea, 2:379, 1937. -L. B. Smith, Contr. U.S. Nat. Herb., 35:267, 1962.Ayensu, Smithsonian Contr. Bot., 15:51, fig. 4e, pl. 46b, 1974 .

TyPE.-Among rocks, Serra da Lapa (Cipó), Minas Gerais, Brazil, November 1824, Riedel 1051 (LE, holotype; L, isotype; $\mathrm{B}$, photo $86, \mathrm{~s} \mathrm{n}$ ).

Distribution.--Brazil: Minas Gerais: rock outcrops, Mun. Datas, $27 \mathrm{~km}$ west of Sêrro on route 


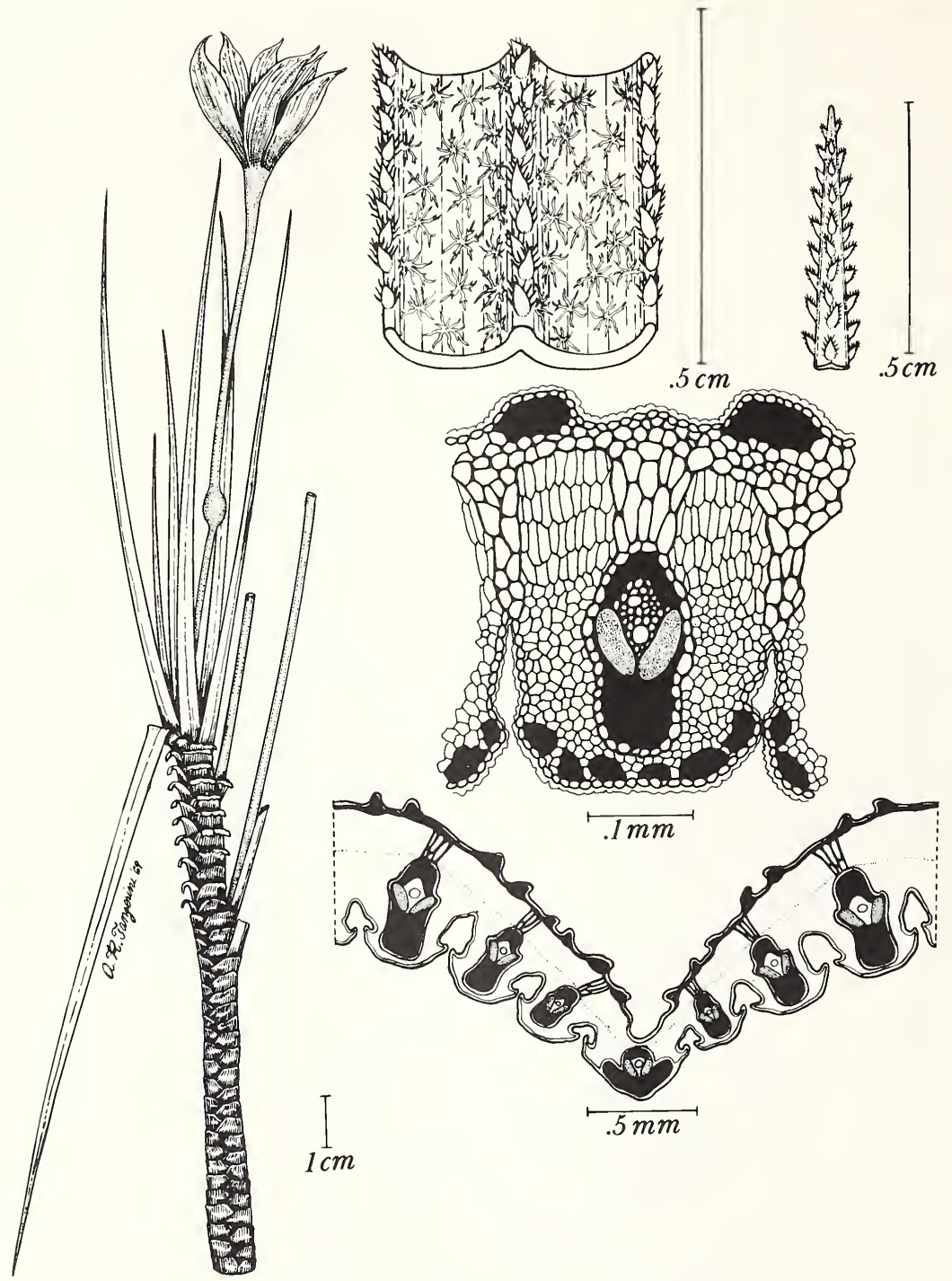

Figure 52.-Vellozia annulata Goethart \& Henrard (Glaziou 22212). 
MG-2, 19 January 1972, L. B. Smith, Ayensu \& Hatschbach (separate no. 28965) 15983 (MBM, US). The type-locality is so vague that this may well be topotypic.

\section{Vellozia dumitiana R. E. Schultes}

Vellozia dumitiana R. E. Schultes, Mutisia, I2:2, unnumbered pl. 1952.-L. B. Smith, Contr. U.S. Nat. Herb., 35: 269, 1962.-Ayensu, Smithsonian Contr. Bot., 15:48, pls. 2a-b, 45d, 1974 .

TyPE.-On sandstone, Cerro Isibukuri, Río Kananari (affluent of Río Apaporis), Vaupés, Colombia, ca. $700 \mathrm{~m}$ alt, 1 December 1951, R. E. Schultes \& Cabrera 14726 (GH, holotype; COL, isotype).

Distribution.-Known only from the typelocality.

\section{Vellozia lithophila $\mathrm{R}$. E. Schultes}

Vellozia lithophila R. E. Schultes, Fam. Velloz. Colomb. in Rev. Acad. Colomb. Cienc., 8(32):459, unnumbered pl., 1950.-L. B. Smith, Contr. U.S. Nat. Herb., 35:270, 1962.Ayensu, Smithsonian Contr. Bot., 15:49, figs. 2b, 16d-f, pl. 2c-d, 1974.

Type.-Mesa La Lindosa, 15-20 km south of San José del Guaviare, Vaupés, Colombia, 400-600 m alt, 13-15 December 1950, Idrobo \& Schultes 632 (GH, holotype; US photo).

Distribution.-Colombia: Meta: San José del Guaviare. Vaupés: Cerro Kañendá, $1^{\circ} 0^{\prime} \mathrm{N}, 70^{\circ} 5^{\prime} \mathrm{W}$; Río Paraná Pichuna, $1^{\circ} 10^{\prime} \mathrm{N}, 70^{\circ} 30^{\prime} \mathrm{W}$; Yapobodá $1{ }^{\circ} 20^{\prime} \mathrm{N}, 70^{\circ} 30^{\prime} \mathrm{W}$.

\section{Vellozia macarenensis Philipson}

Figure 53

Vellozia macarenensis Philipson in Schultes, Fam. Velloz. Colomb. in Rev. Acad. Colomb. Cienc., 8(32):461, unnumbered pl., 1952.-L. B. Smith, Contr. U.S. Nat. Herb., 35: $268,1962$.

Leaf ANatomy (Garcia-Barriga et al. 17032, Thomas et al. 1596, Philipson et al. 2320).-Surface View: Hairs: present in tufts on both surfaces; short hairs on adaxial; long on abaxial. Epidermis: cells square to rectangular; thin-walled. Stomata: paracytic, some tetracytic, $18 \times 12 \mu \mathrm{m}$; present in abaxial furrows only.

Transverse Section of Lamina: Dorsiventral;

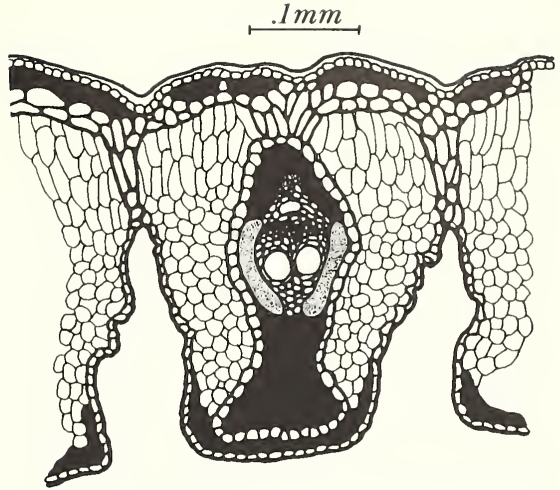

Figure 53.-Vellozia macarenensis Philipson (Philipson, Idrobo \& Jaramillo 2320).

widely V-shaped with margins curved downward and partially backward. Adaxial surface ridged; abaxial surface furrowed $1 / 2$ to $3 / 4$ thickness of blade. Hairs present on both surfaces. Epidermis: cells on both surfaces rounded to dome-shaped; thinwalled. Large amount of abaxial cells replaced by sclerenchyma fibers. Subjacent to adaxial epidermis is a layer of thin-walled cells interspersed with sclerenchyma fibers. This is followed by a layer of large, thin-walled cells. Cuticle: thick and smooth on adaxial surface; thinner and smooth on abaxial surface. Stomata: present in abaxial furrows only; stomata raised above surface and have a small substomatal chamber. Mesophyll: 3 or 4 layers of palisade cells followed by compactly arranged spongy mesophyll. Translucent palisade cells radially arranged above vascular bundles and furrows. Sclerenchyma fibers present on abaxial corners of furrows and extending partially into furrows. Vascular bundles: 15-27; commissural bundles not observed. One or two large vessels present in each bundle, mostly one. Two phloem units lying laterally in flanges of Y-shaped abaxial girder. Adaxial cap present on each bundle. Bundles surrounded by a bundle sheath. Crystals: none observed. Tannins: few present.

TYPE.-Open space on summit of knoll, Sierra de La Macarena, northern escarpment, Meta, Co- 
lombia, $900 \mathrm{~m}$ alt, l February 1950, Philipson, Idrobo \& Jaramillo 2320 (BM, holotype; COL, GH, isotypes).

Distribution.-Known only from the type-area.

\section{Vellozia rhynchocarpa Goethart \& Henrard}

Vellozia rhynchocarpa Goethart \& Henrard, Blumea, 2:378, 1937.-L. B. Smith, Contr. U.S. Nat. Herb., 35:269, 1962.

TyPE.-Dry rocky campo, near Mato Grosso between Cana Verde and Cajuru, $21^{\circ} 17^{\prime} \mathrm{S}, 47^{\circ} 20^{\prime} \mathrm{W}$, São Paulo, Brazil, February 1849, Regnell III-1241 (L, holotype; S, US, isotypes).

Distribution.-Known only from the typecollection.

\section{Vellozia tubiflora (A. Richard) Humboldt, Bonpland, \& Kunth}

Vellozia tubiflora (A. Richard) Humboldt, Bonpland, \& Kunth, Nov. Gen. \& Sp., 7:155, 1825.-L. B. Smith, Contr. U.S. Nat. Herb., 35:270, 1962.-Ayensu, Smithsonian Contr. Bot., 15:51, fig. 17a-c, pls. 35e-h, 38a-b, 1974.

Radia tubiflora A. Richard in Kunth, Syn. P1. Aeq., 1:300, 1822.

Barbacenia alexandrinae Schomburgk, Barbacenia alexandrinae, 13, unnumbered pl., 1845.-Schomburk ex Hooker, London Journ. Bot., 4:13, 1845 [type: on sandstone hills, Mount Roraima, Guyana, 900-1200 m (3-4,000 ft) alt, 1835-43, Robert H. Schomburgk (K, B, F, photo 10050)].

Barbacenia tubiflora (A. Richard) Jackson, Ind. Kew, 4:1269, 1895 [wrongly attributed to Bentham \& Hooker, Gen., $3: 740,1883$, who made the combination only inferentially and not validly].

?Vellozia macrosiphonia Taubert, Bot. Jahrb., 21:424, 1896 [type: Serra dos Pirineus, Serra Dourada and Serra dos Veadeiros, Goiás, Brazil, Ule 3142 (B, holotype n v); the size of flowers of $V$. tubiflora varies widely enough to include $V$. macrosiphonia, and $V$. tubiflora has been collected in Cristalina, Goiás, south of the stated area of $V$. macrosiphonia].

Vellozia alexandrinae (Schomburgk) Goethart \& Henrard, Blumea, 2:363, 1937.

?Vellozia duidae Steyermark, Fieldiana Bot., 28(1):157, 1951 [type: ridge top, Savana Hills, summit of Cerro Duida, Amazonas, Venezuela, 1025-1200 m alt., 2 September 1944, Steyermark 58270 (F, sterile)].

Type.-On granite rock, Rio Orinoco, Venezuela, May 1800, Humboldt \& Bonpland s $n$ (P, holotype $\mathrm{n}$ v).

Distribution.-Colombia: Vichada: Raudal Atures, Raudal San Borja, on Río Orinoco. Vene- zuela: Bolivár: Auyan-tepui, Cerro Moriche, Kavanayen, Luepa. Amazonas: Duida (Cerro), Puerto Ayacucho, Sanariapo, Yutaje (Cerro). Guyana: Essequibo: Karaurieng River, Partang River. Brazil: Goiás: Cristalina.

\section{Excluded and Doubtful Taxa}

\section{Barbacenia gracilis hortus ex Baker}

Barbacenia gracilis hortus ex Baker, Journ. Linn., 18:239, 1880 [nomen].

This horticultural name was published in the synonymy of Dasylirion acrotrichum Zuccarini, a liliaceous species of Mexico.

\section{Barbacenia uaipanensis Maguire}

Barbacenia uaipanensis Maguire, Mem. N. Y. Bot. Gard., 9:477, fig. 117, 1957.

This species belongs in the Bromeliaceae. See Ayensua uaipanensis (Maguire) L. B. Smith (Mem. N.Y. Bot. Gard., 18(2):29, fig. 5A,B, 1969, and Ayensu, Mem. N.Y. Bot. Gard., 18(2):291-298).

\section{Vellozia capsulis glabris, scapis hispidis Vandelli}

Vellozia capsulis glabris, scapis hispidis Vandelli, Fl. Lusit. \& Brasil. Spec., 32, pl. 2, 1788.

The above is interpreted as a polynomial because the words are separated from the description by a dash and the word for capsule is repeated; this would not occur if the final phrase were part of the description. The species was validated as Vellozia subscabra Mikan (Delect. Fl. \& Faun. Brasil., fasc. 2, 1820) and named again as Vellozia scabra Sprengel (Syst. 3:338, 1826). However, the identity of the species remains a mystery because in nearly 200 years of subsequent collecting in Minas Gerais there is yet to be found a Vellozia with a vestite scape and naked ovary, although Vandelli's other species is well known.

\section{Vellozia coerulescens hortus Belg. ex Gumbl.}

Vellozia coerulescens hortus Belg. ex Gumbl., Gard. Chron. for $1874,2: 623,657,1874$. 
According to Jackson (Index Kewensis 4:1173, 1895), this horticultural name is a synonym of Barbacenia purpurea Hooker.

\section{Vellozia leptophylla Seubert}

Vellozia leptophylla Seubert in Martius, F1. Bras., 3(1):84, 1847.

This species was based on a sterile collection by Sellow without any indication of locality.

\section{Vellozia macrantha Lemaire}

Vellozia macrantha Lemaire, Ill. Hortic., 12(misc.):41, 1865.

This species was placed in section Radia by L. B. Smith (1962:268), but under our new and narrower interpretation it can scarcely remain in that section and its placement elsewhere seems very doubtful.

\section{Vellozia scabra Sprengel}

Vellozia scabra Sprengel, Syst., 3:338, 1826.

Unidentifiable, see under Vellozia capsulis glabris, scapis hispidis.

\section{Vellozia squamata auctt. ex Steudel}

Vellozia squamata auctt. ex Steudel, Nom. Bot. ed. 2, 2:746, 1841 [nomen].
This name was published in the synonymy of Xerophyllum sabadilla, a Mexican liliaceous species of doubtful identity. It is scarcely more than evidence of a misdetermination.

\section{Vellozia subscabra Mikan}

Vellozia subscabra Mikan, Delect. F1. \& Faun. Brasil., fasc. $2,1820$.

See under Vellozia capsulis glabris, scapis hispidis.

\section{Vellozia tomentosa Pohl}

Vellozia tomentosa Pohl, Pl. Bras., 1:130, 1827.

This species was described from sterile material collected between the crossing of the Rio Jequitinhonha and Calumbí, Minas Gerais, Brazil. It may be possible to identify it yet by leaf anatomy, in which case it may well antedate a name now in use.

Vellozia uaipanensis (Maguire) L. B. Smith

Vellozia uaipanensis (Maguire) L. B. Smith, Contr. U.S. Nat. Herb., 35:267, 1962.

See under Barbacenia uaipanensis Maguire. 


\section{Literature Cited}

Ayensu, E. S.

1968. The Anatomy of Barbaceniopsis, a New Genus Recently Described in the Velloziaceae. American Journal of Botany, 55:399-405.

1972. Studies on Pollen Morphology in the Velloziaceae. Proceedings of the Biological Society of Washington, 85:469-472.

1973. Biological and Morphological Aspects of the Velloziaceae. Biotropica, 5(3):135-149.

1974. Leaf Anatomy and Systematics of New World Velloziaceae. Smithsonian Contributions to Botany, $15: 1-125$.

Ayensu, E. S., and Skvarla, J. J.

1974. Fine Structure of Velloziaceae Pollen. Bulletin of the Torrey Botanical Club, 101(5):250-266.

Carlquist, $\mathrm{S}$.

1969. Toward Acceptable Evolutionary Interpretations of Floral Anatomy. Phytomorphology, 19:332-362, figures 1-20.
Greves, Susie

1921. A Revision of the Old World Species of Vellozia. Journal of Botany, 59(706):273-284.

Maguire, B.

1969. Velloziaceae. Pages $32-41$ in B. Maguire, editor, Botany of the Guayana Highland. Memoirs of the New York Botanical Garden, 18(2):1-291.

Menezes, N. L. de

1971a. Traqueides de Transfusão no Gênero Vellozia Vand. Ciência e Cultura, 23(3):389-409.

1971b. New Taxa and New Combinations in Velloziaceae. Ciência e Cultura, 23(3)[421]-422.

Noher de Halac, R., and Cocucci, A. E.

1971. Sobre la Naturaleza de los "appendices petaloides" en Barbacenia purpurea (Velloziaceae). Kurtziana, 6:265-269.

Smith, L. B.

1962. A Synopsis of the American Velloziaceae. Contributions from the United States National Herbarium, $35: 251-292$. 
Plates 



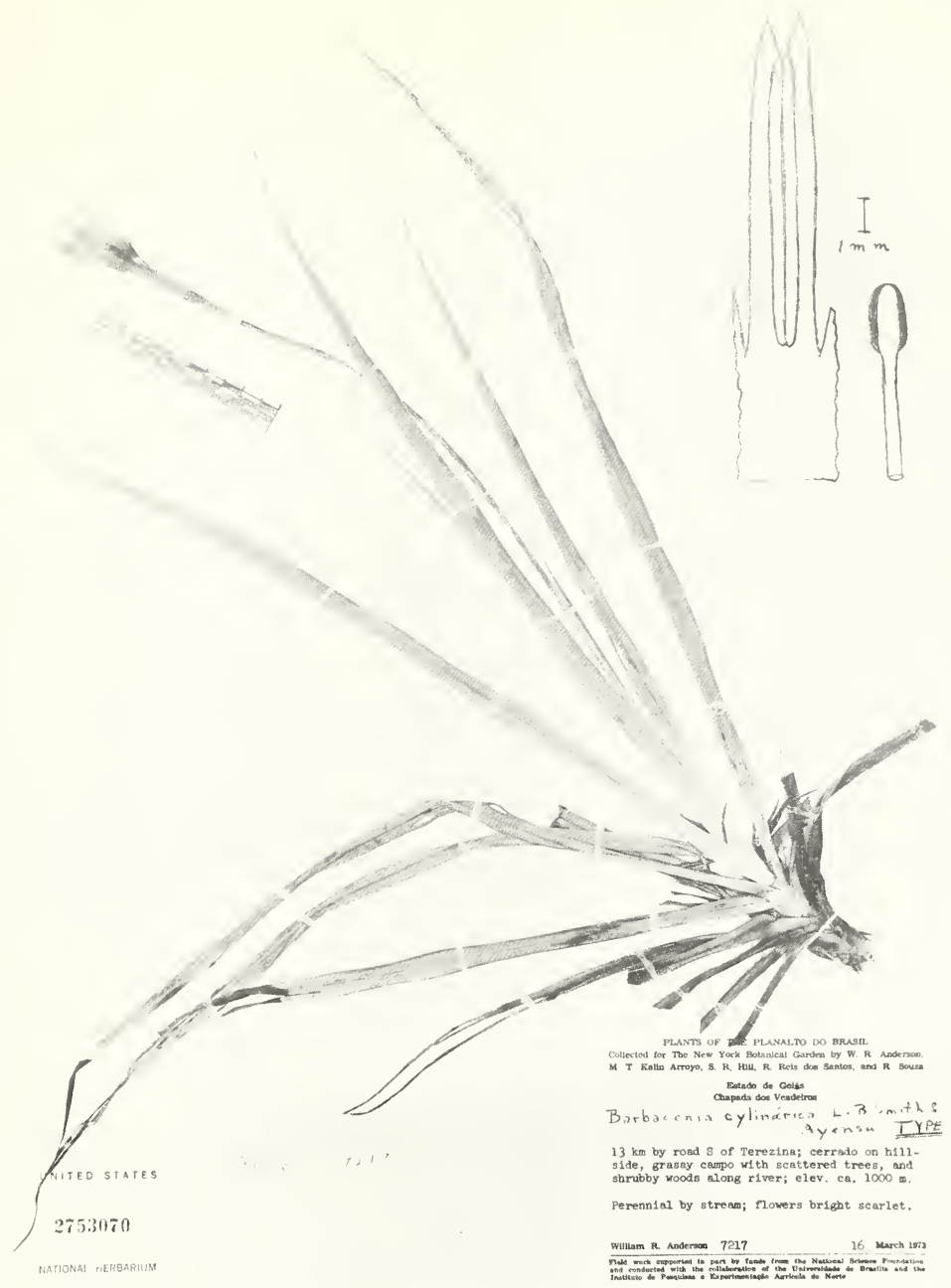

Plate 1.-Barbacenia cylindrica L. B. Smith \& Ayensu, new species (Anderson 7217). 


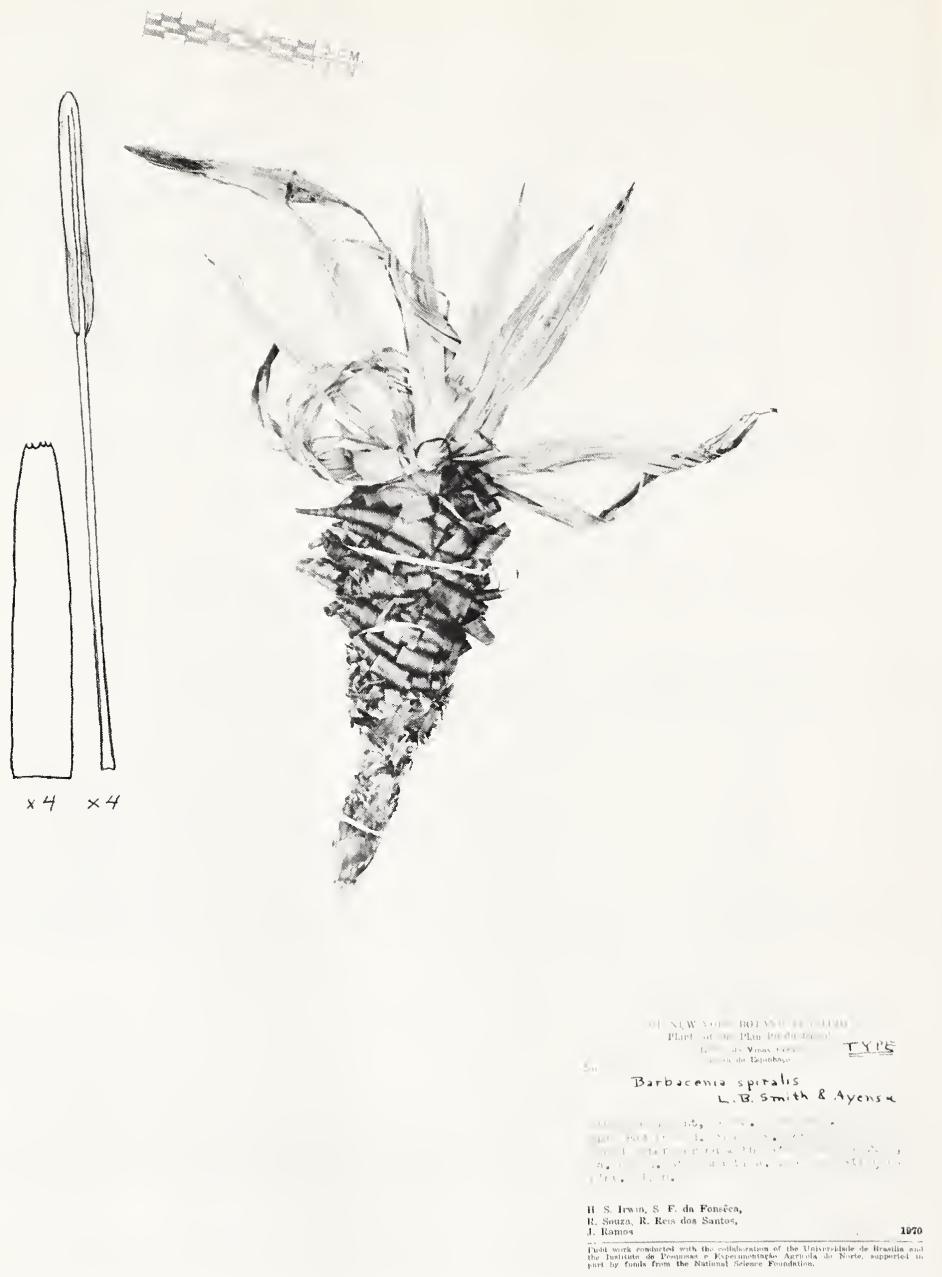

Plate 2.-Barbacenia spiralis L. B. Smith \& Ayensu, new species (Irwin et al. 27642). 


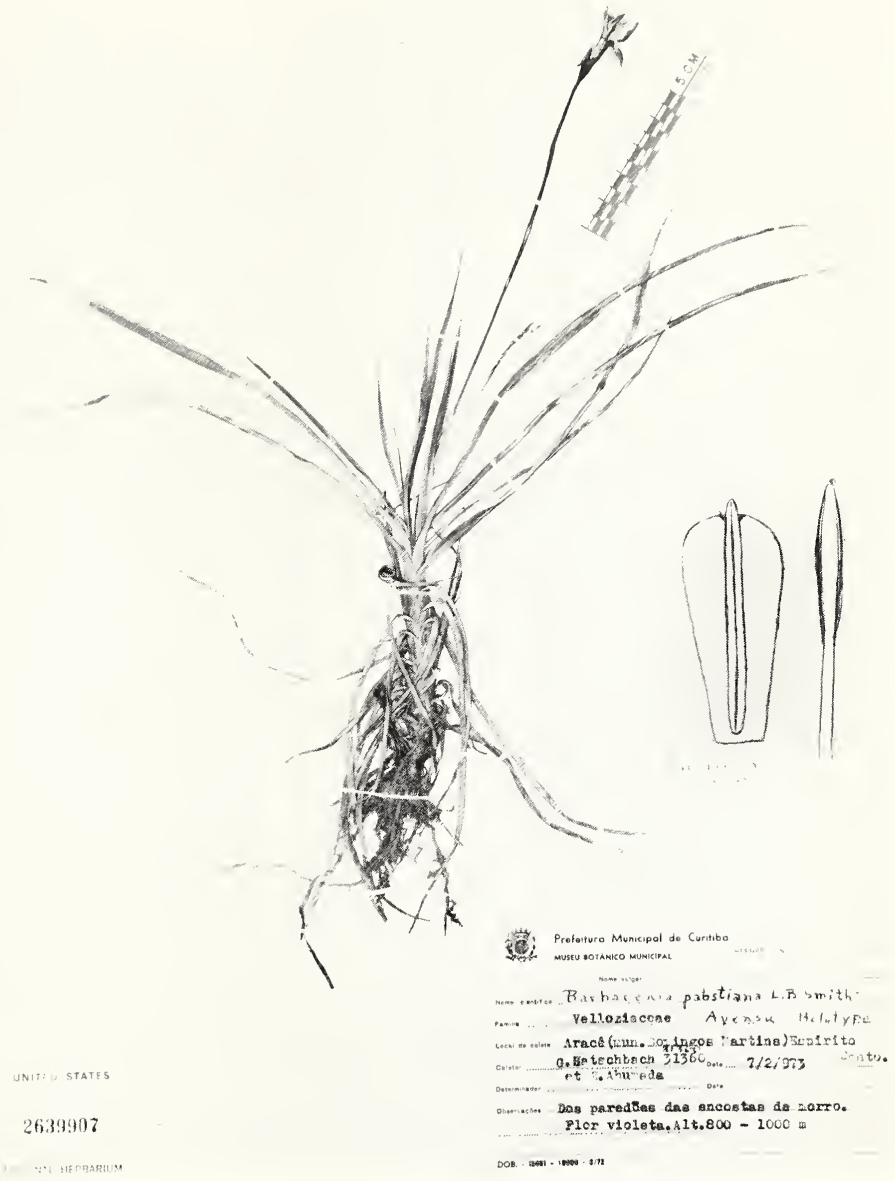

PlATE 3.-Barbacenia pabstiana L. B. Smith \& Ayensu, new species (Hatschbach is Ahumada 31360). 


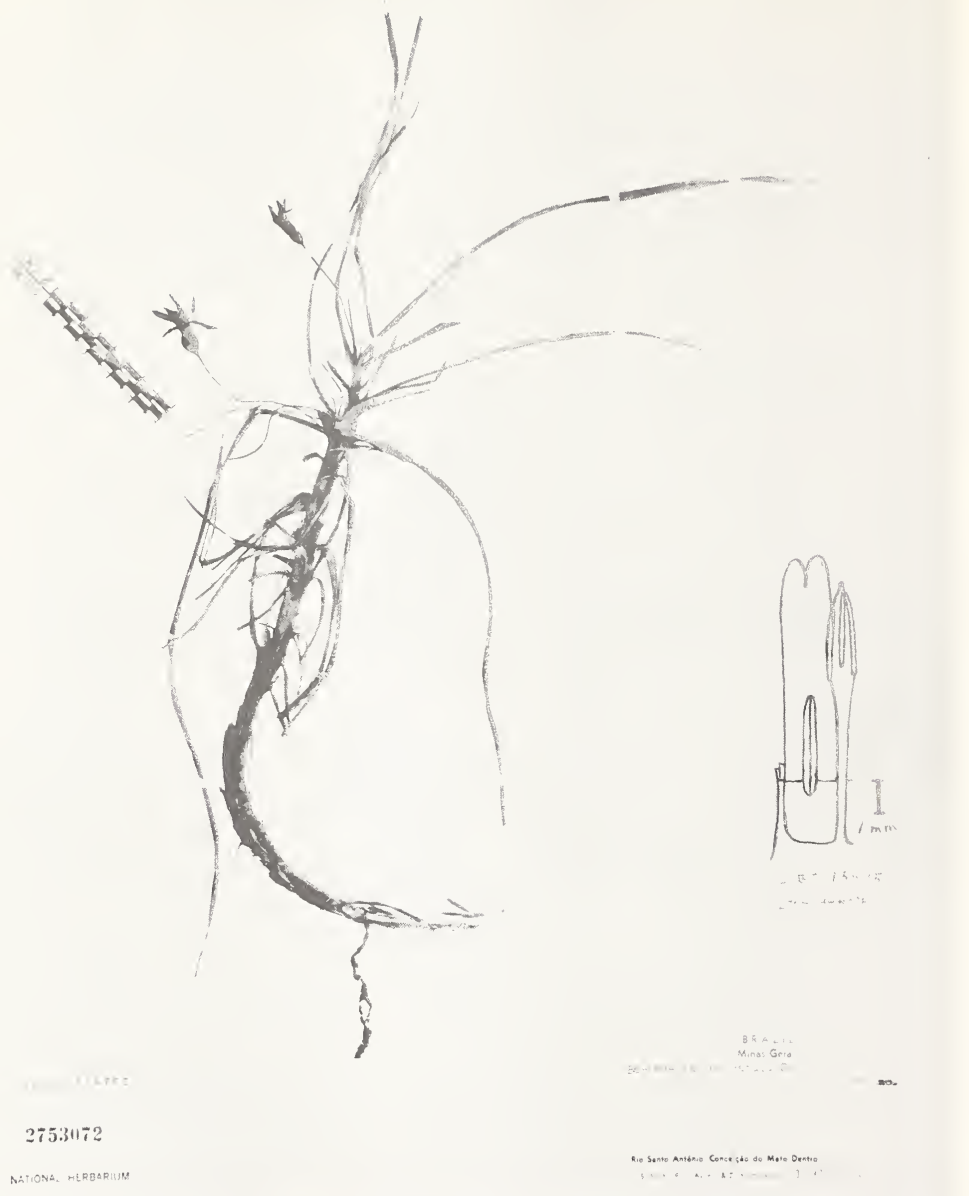

Plate 4.-Barbacenia delicatula L. B. Smith \& Ayensu, new species (L. B. Smith, Ayensu \& Hatschbach 15975). 


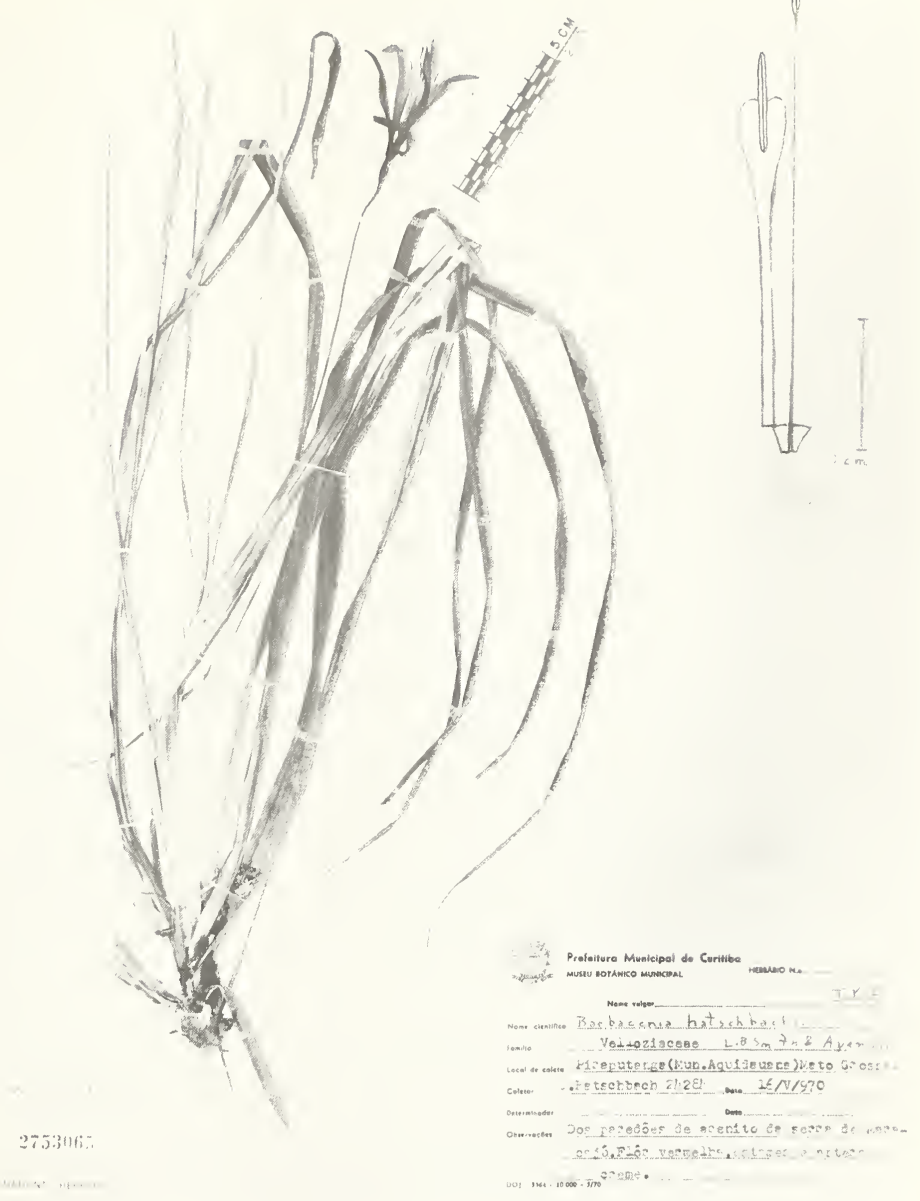

Plate 5.-Barbacenia hatschbachii L. B. Smith \& Ayensu, new species (Hatschbach 24284). 


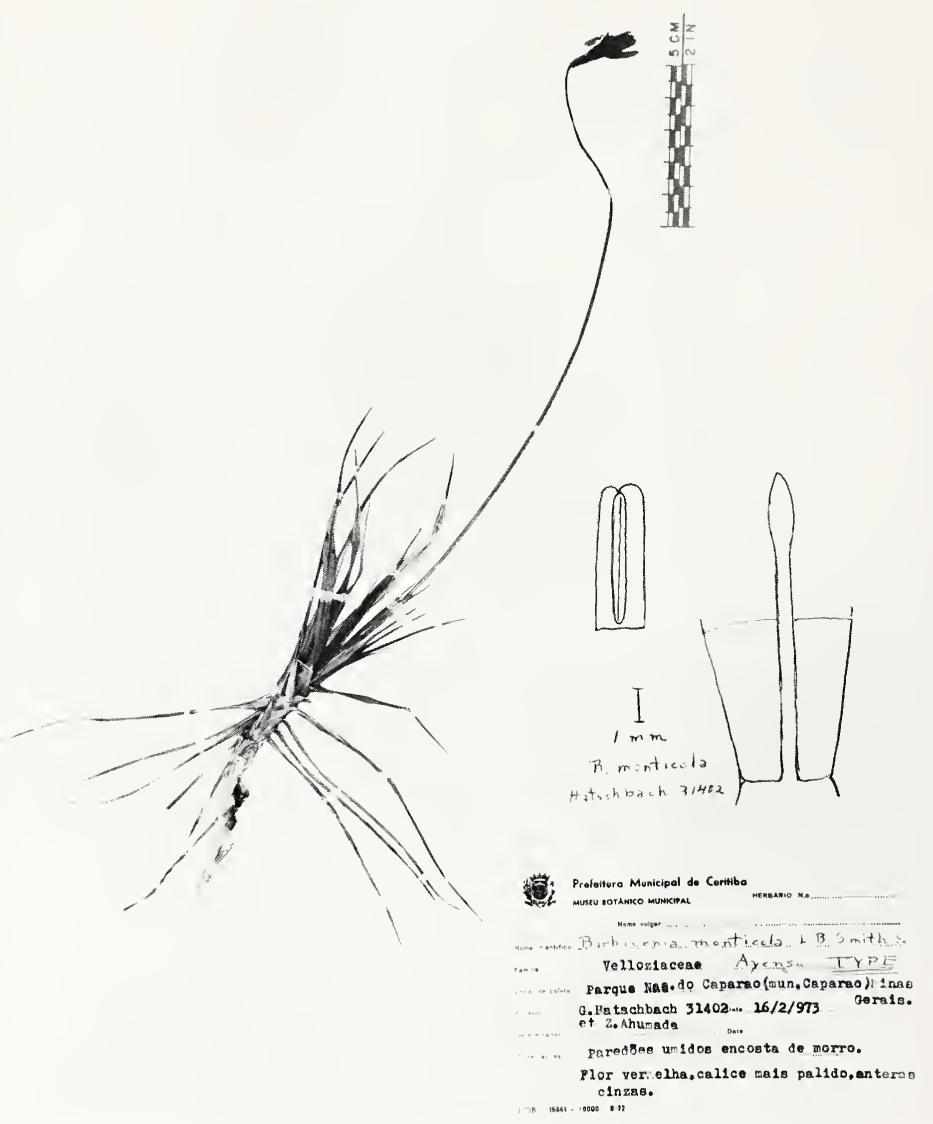

Plate 6.-Barbacenia monticola L. B. Smith \& Ayensu, new species (Hatschbach \& Ahumada 31402). 


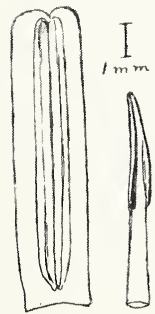

UNITED STATES

2753071

DHONAC HLABARIUM
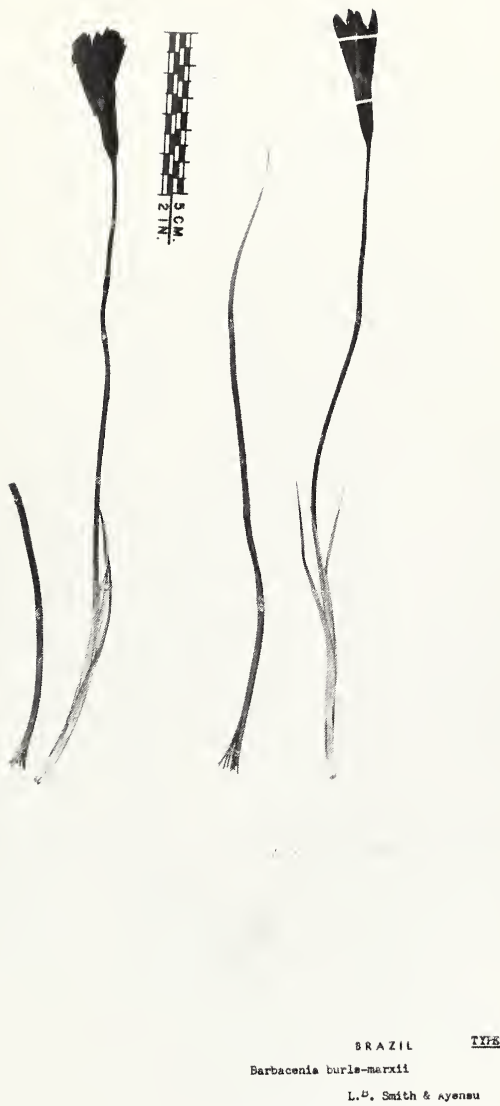

ESPIRITO SAMTO: Venda Nova.

Roberto Furle Msrx cult.

L. B. Soith $\&$ E. S. Ayensu 16014

30 January 1972

Plate 7.-Barbacenia burle-marxii L. B. Smith \& Ayensu, new species (L. B. Smith of Ayensu 16014). 


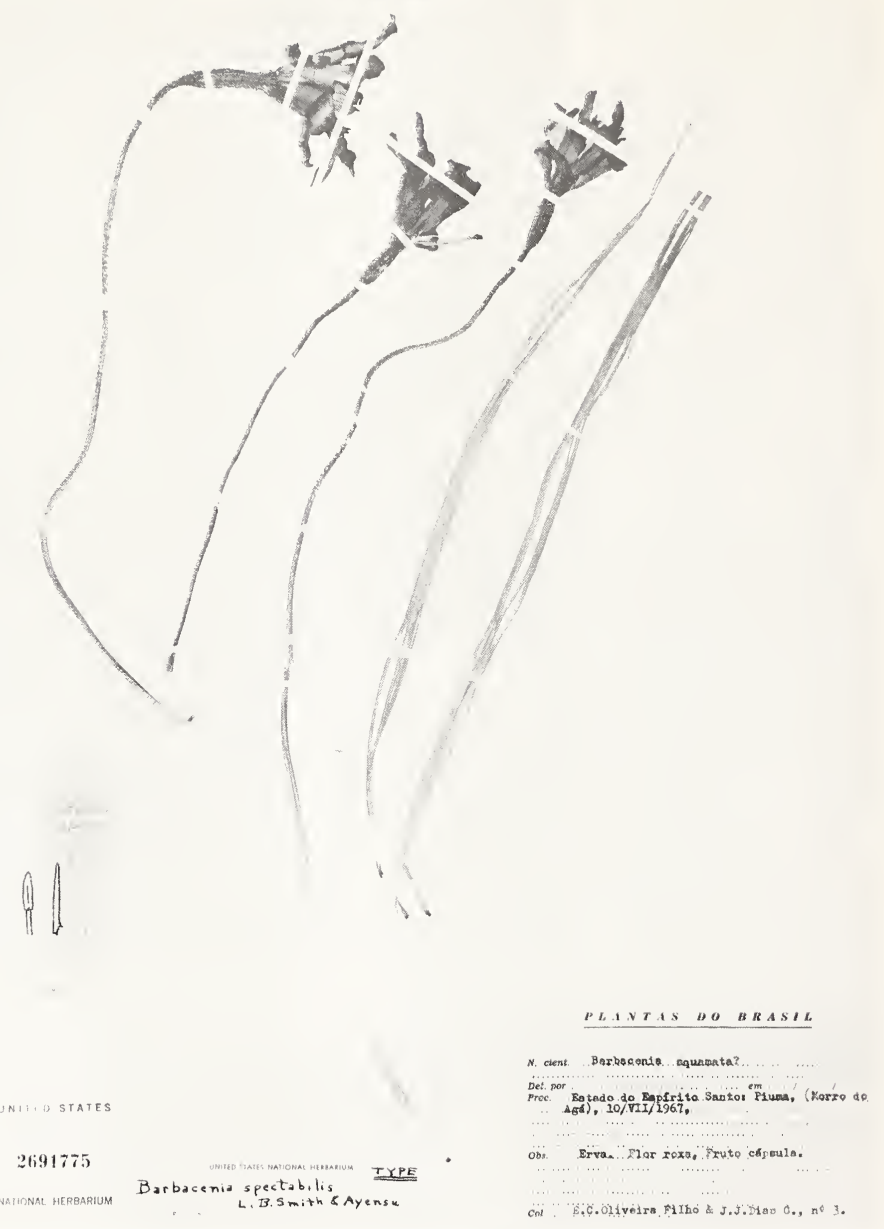

Plate 8.-Barbacenia spectabilis L. B. Smith \& Ayensu, new species (Oliveira Filho \& Dias 3). 


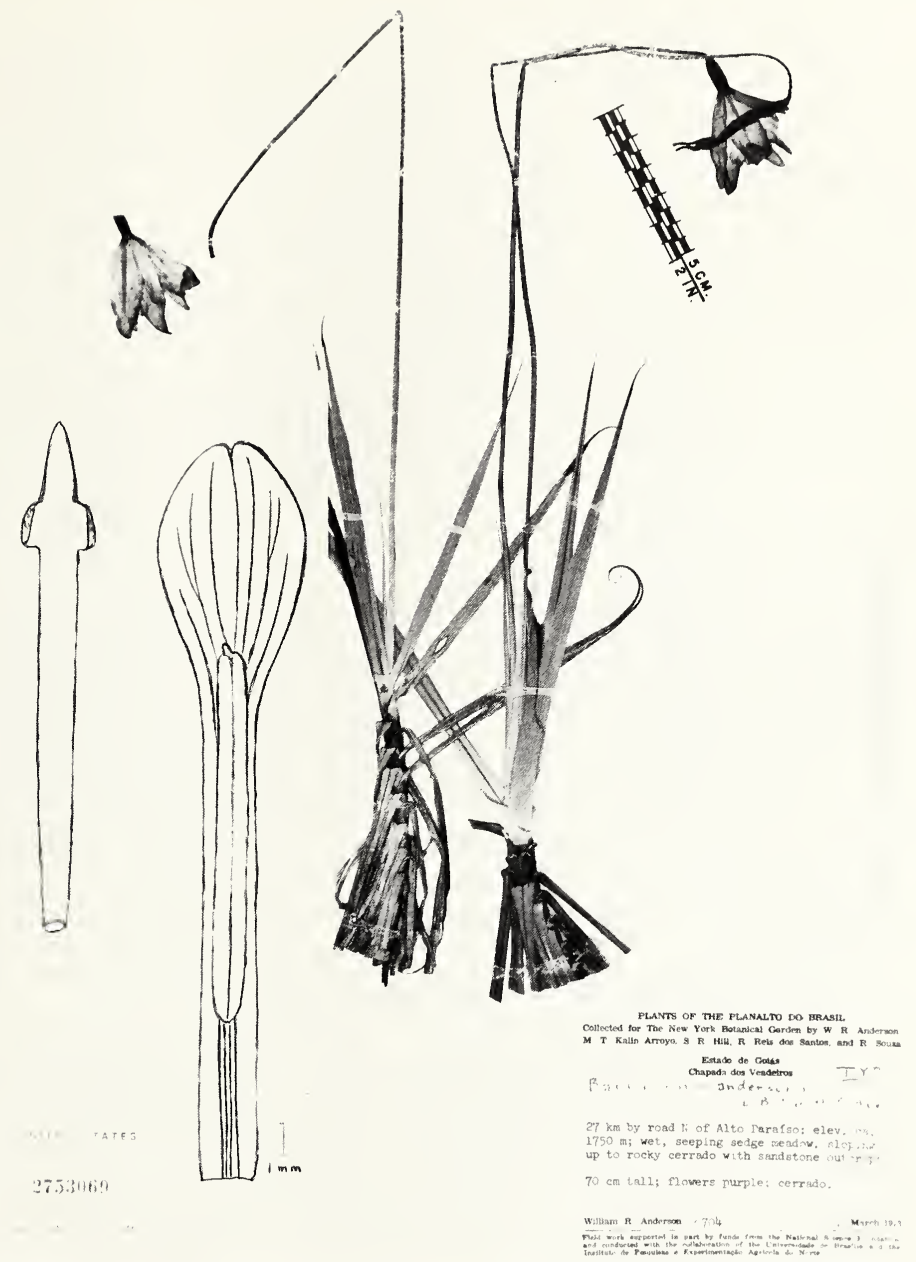

Plate 9.-Barbacenia andersonii L. B. Smith \& Ayensu, new species (Anderson 6704). 


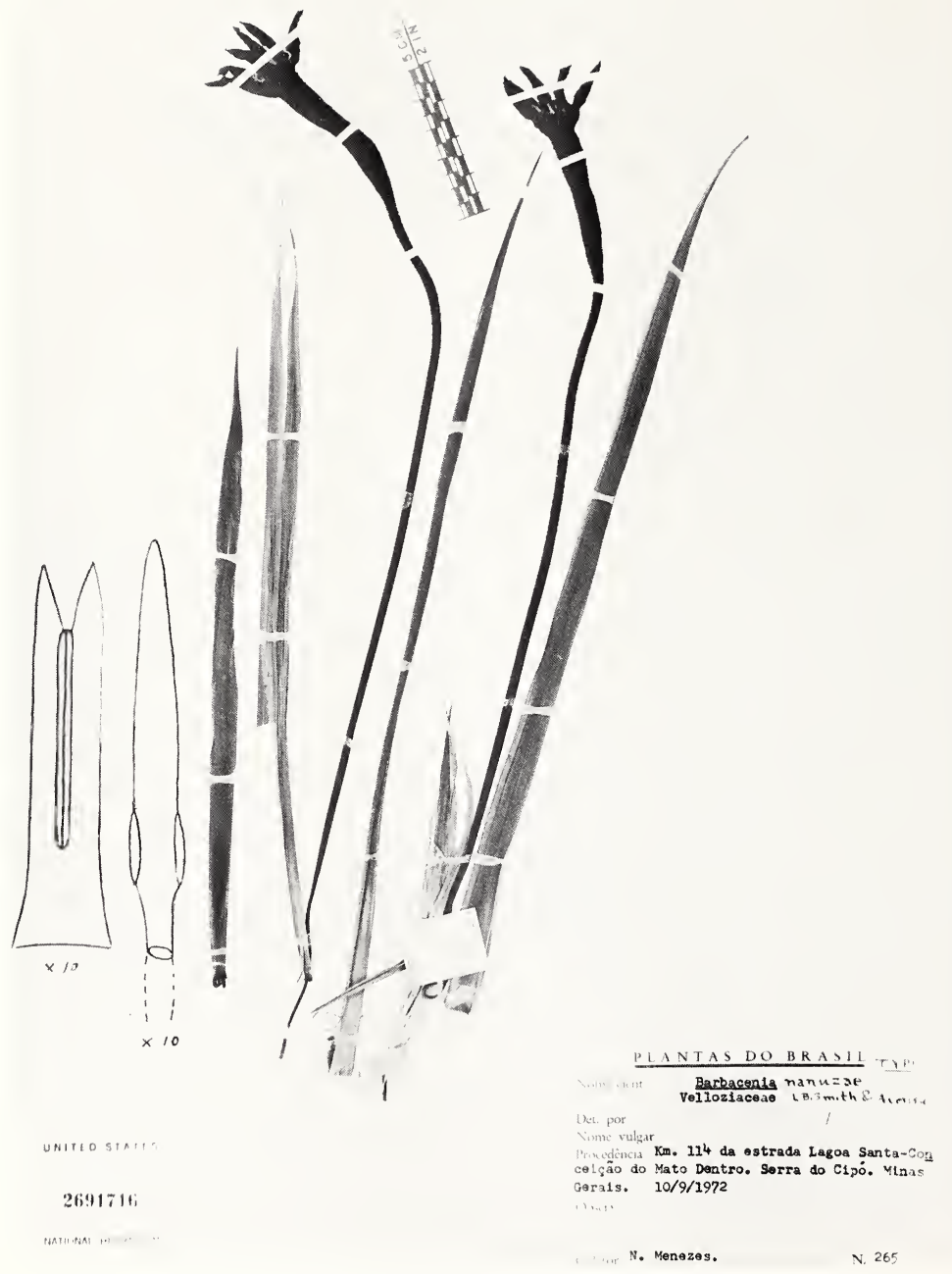

Plate 10.-Barbacenia nanuzae L. B. Smith \& Ayensu, new species (Menezes 265). 

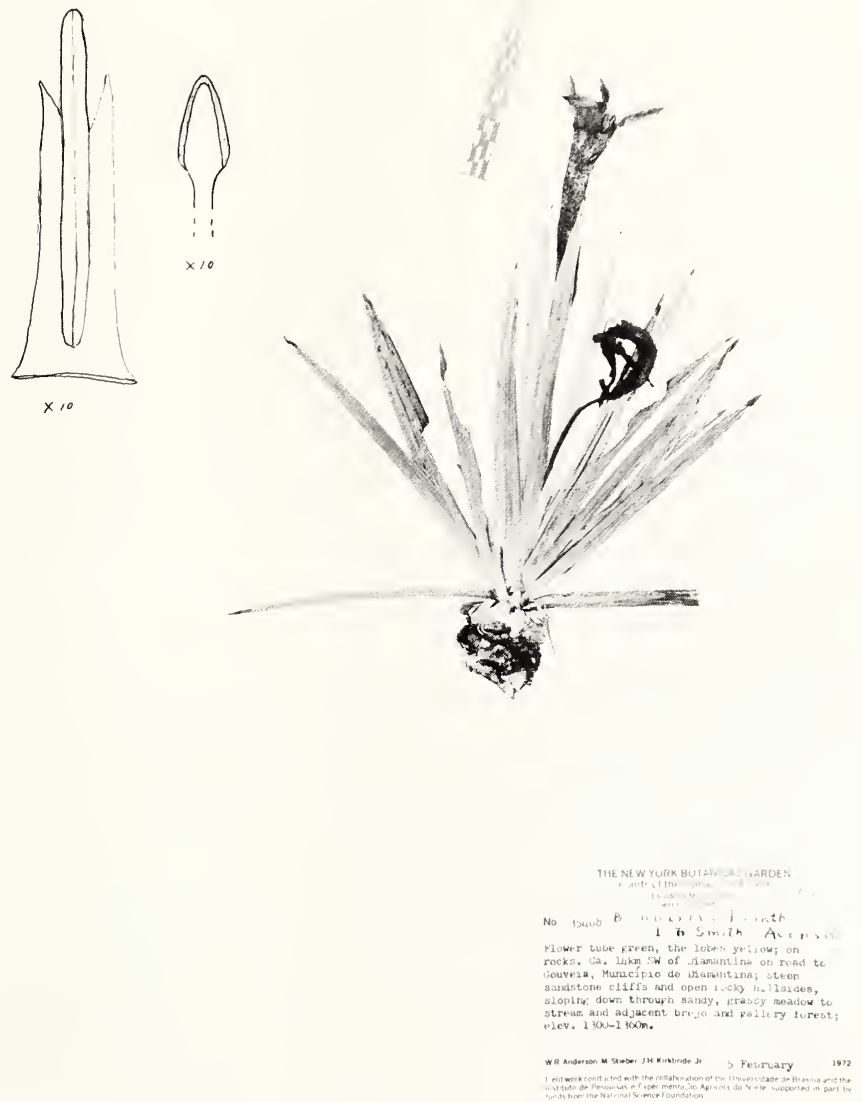

Plate 11.-Barbacenia chlorantha L. B. Smith \& Ayensu, new species (Anderson et al. 35488). 


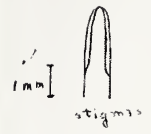

UNITED STATFS

$2645: 349$
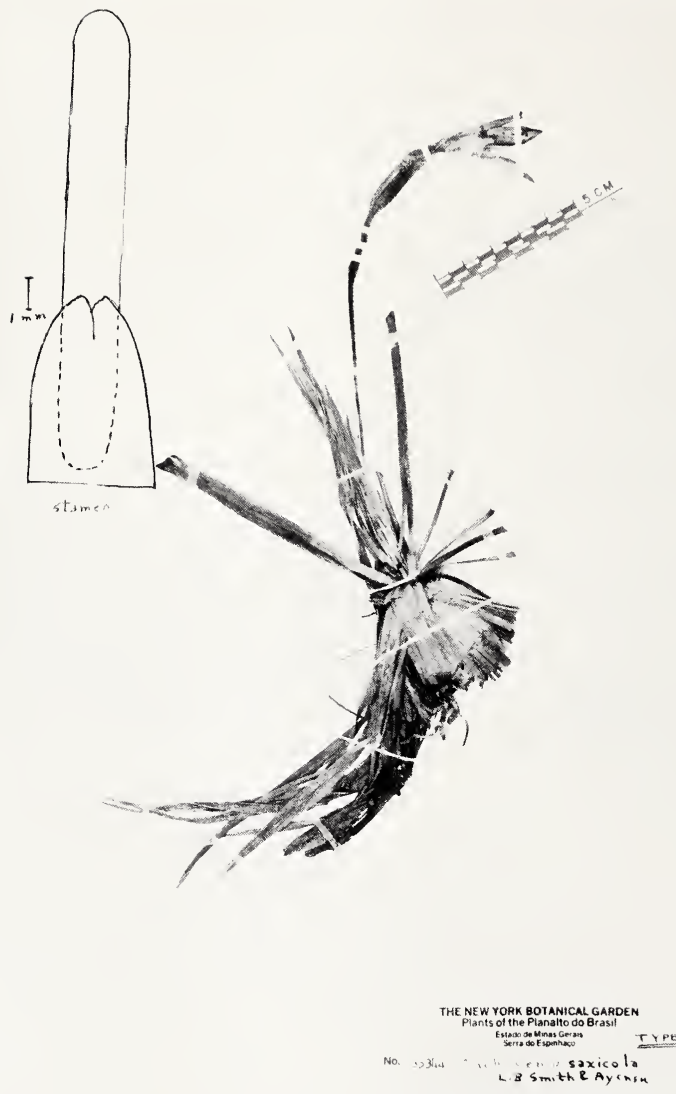

rouks; corolla reddish orange; cerrado.

$k m$ ע. of Gouvela on road to Mamant: na;

v. 1220m. Rocky (sandstone) cerrad a.t.

f. rocky hillsides with wite sandy scil,

A we down to ersssy brejo, creek, and

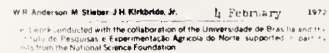

Plate 12.-Barbacenia saxicola L. B. Smith \& Ayensu, new species (Anderson et al. 35344). 


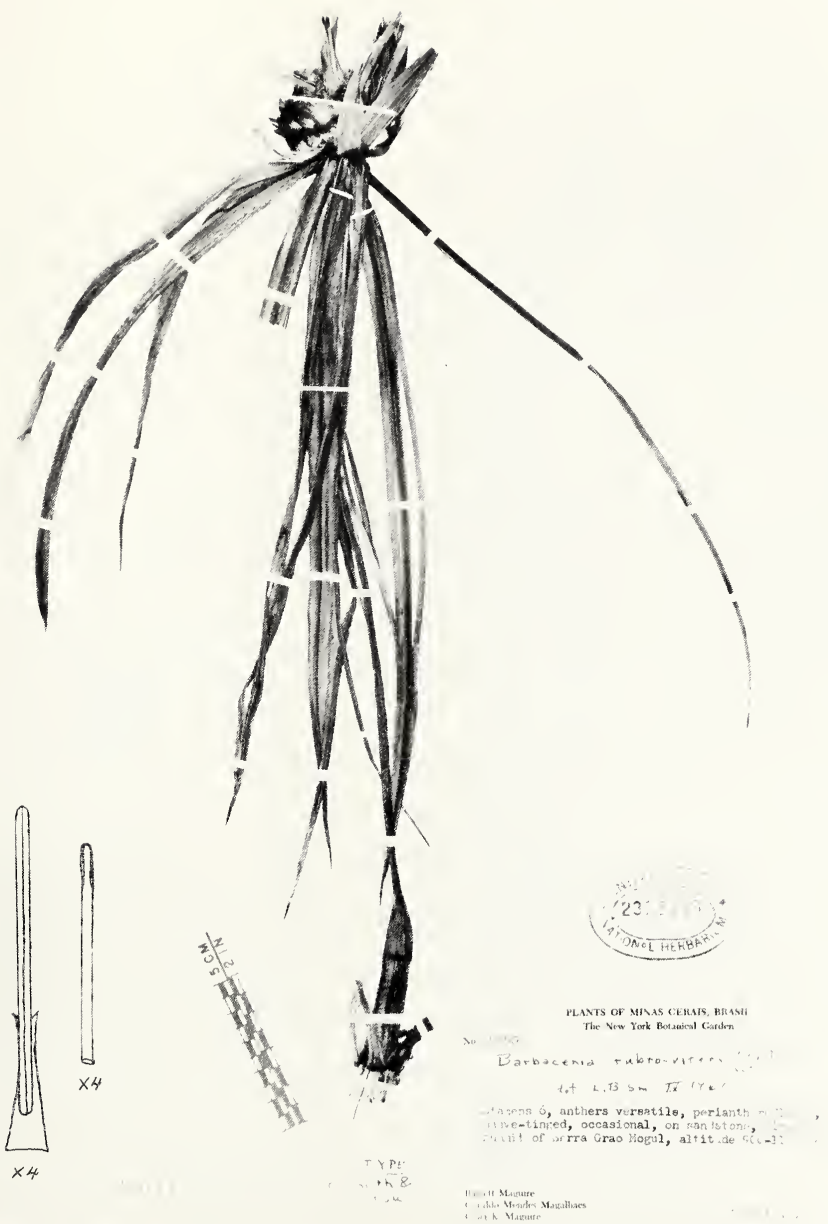

PLaTE 13.-Barbacenia refiexa L. B. Smith \& Ayensu, new species (Maguire et al. 49260). 


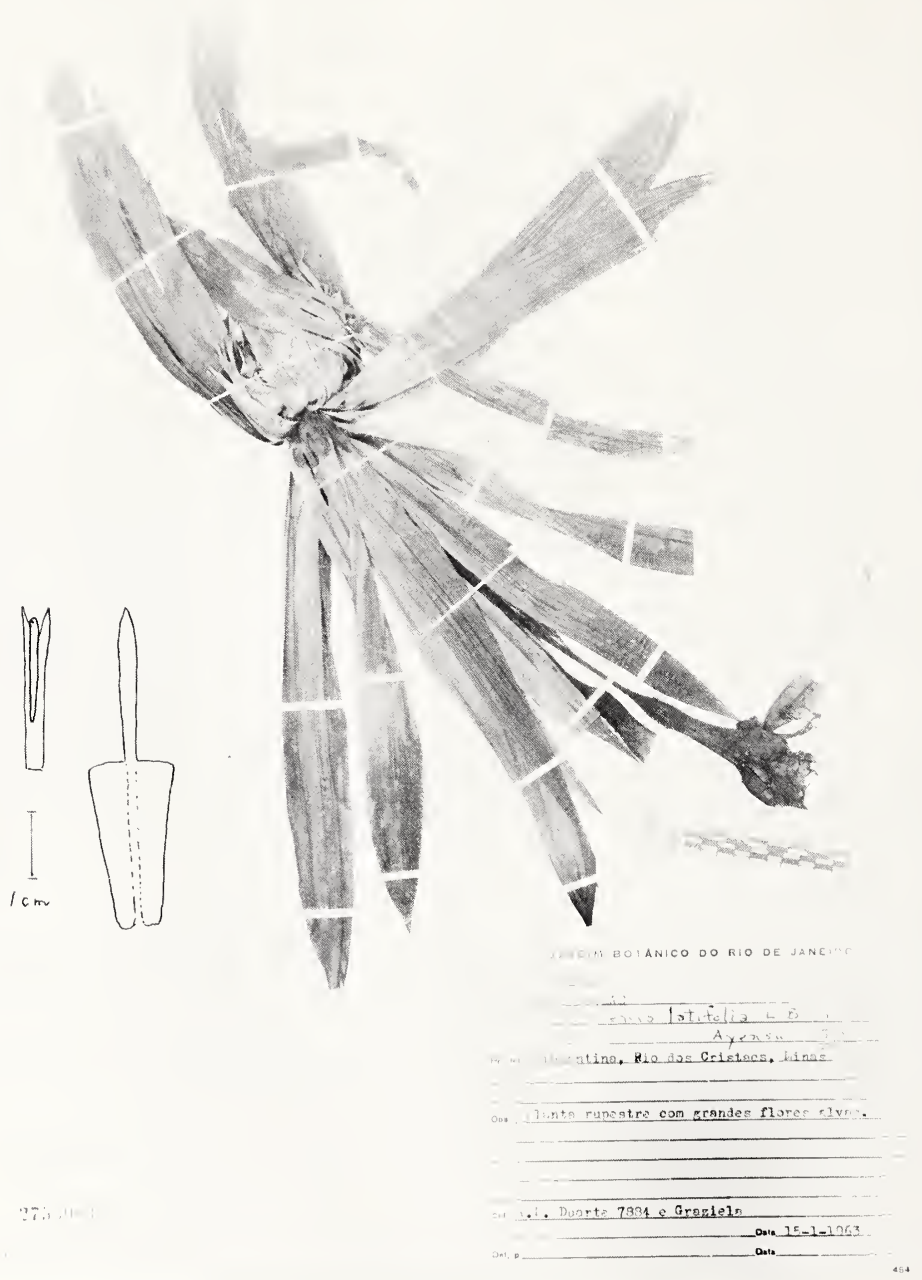

Plate 14.-Barbacenia latifolia L. B. Smith \& Ayensu, new species (Duarte \& Graziela 7884). 


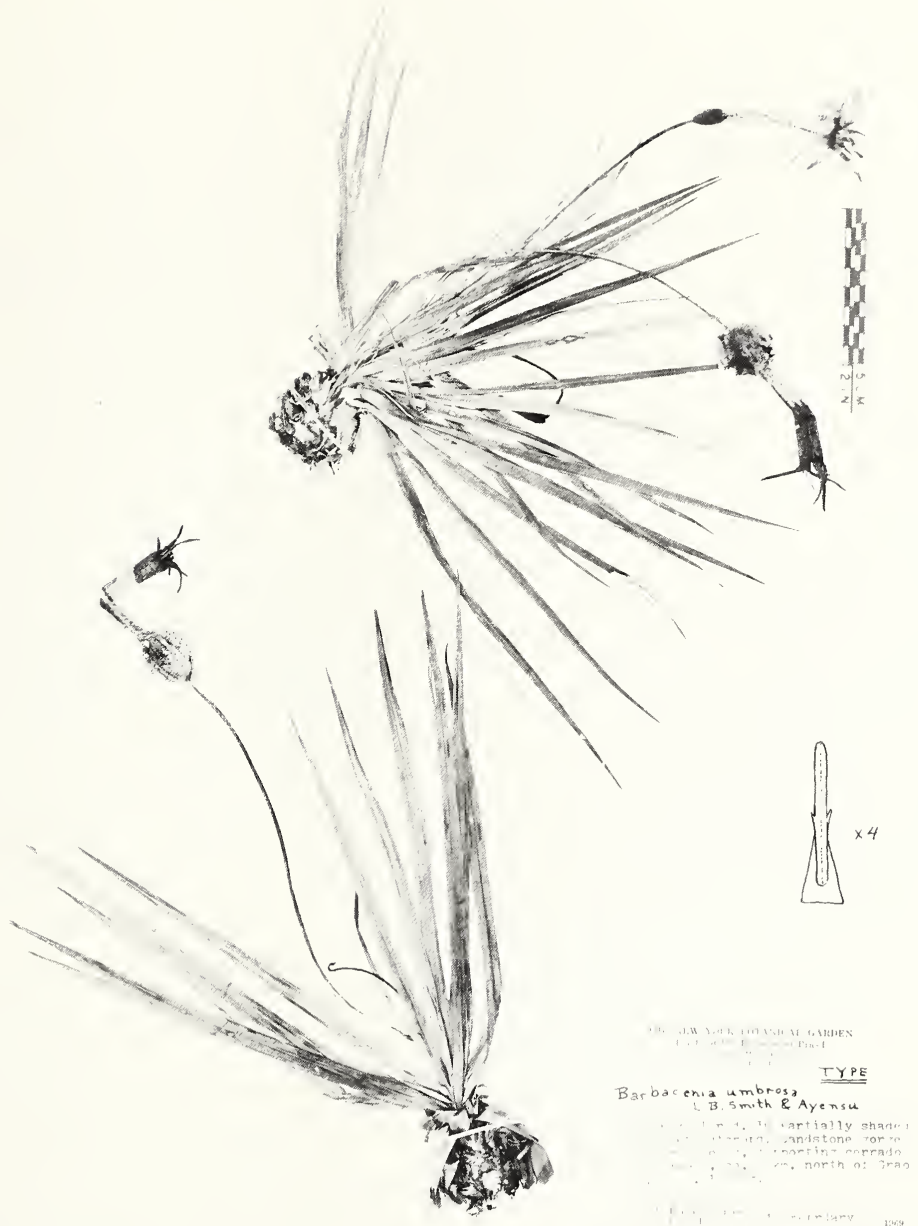

Plate 15.-Barbacenia umbrosa L. B. Smith \& Ayensu, new species (Irwin et al. 23491). 


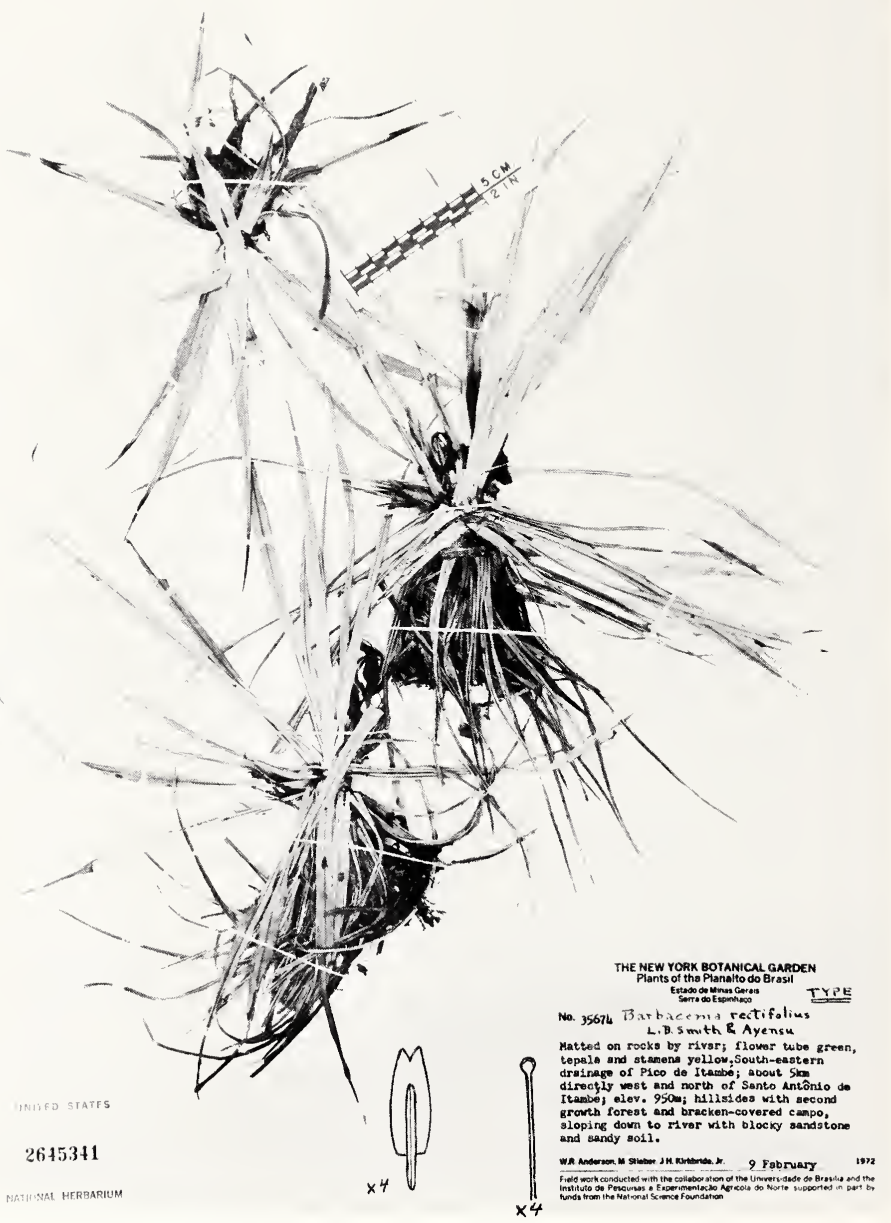

Plate 16.-Barbacenia rectifolia L. B. Smith \& Ayensu, new species (Anderson et al. 35674). 

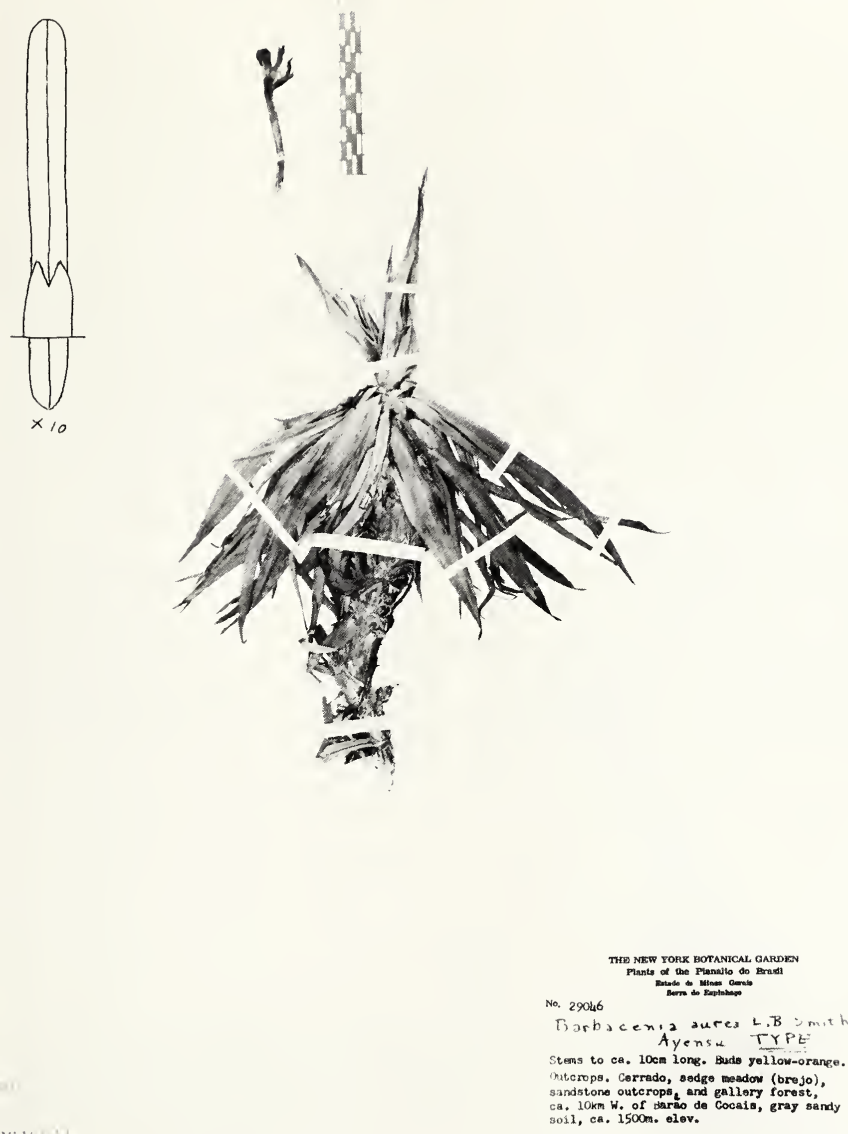

$?: 16911$

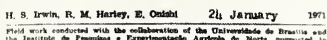

Plate 17.-Barbacenia aurea L. B. Smith \& Ayensu, new species (Irwin et at. 29046). 


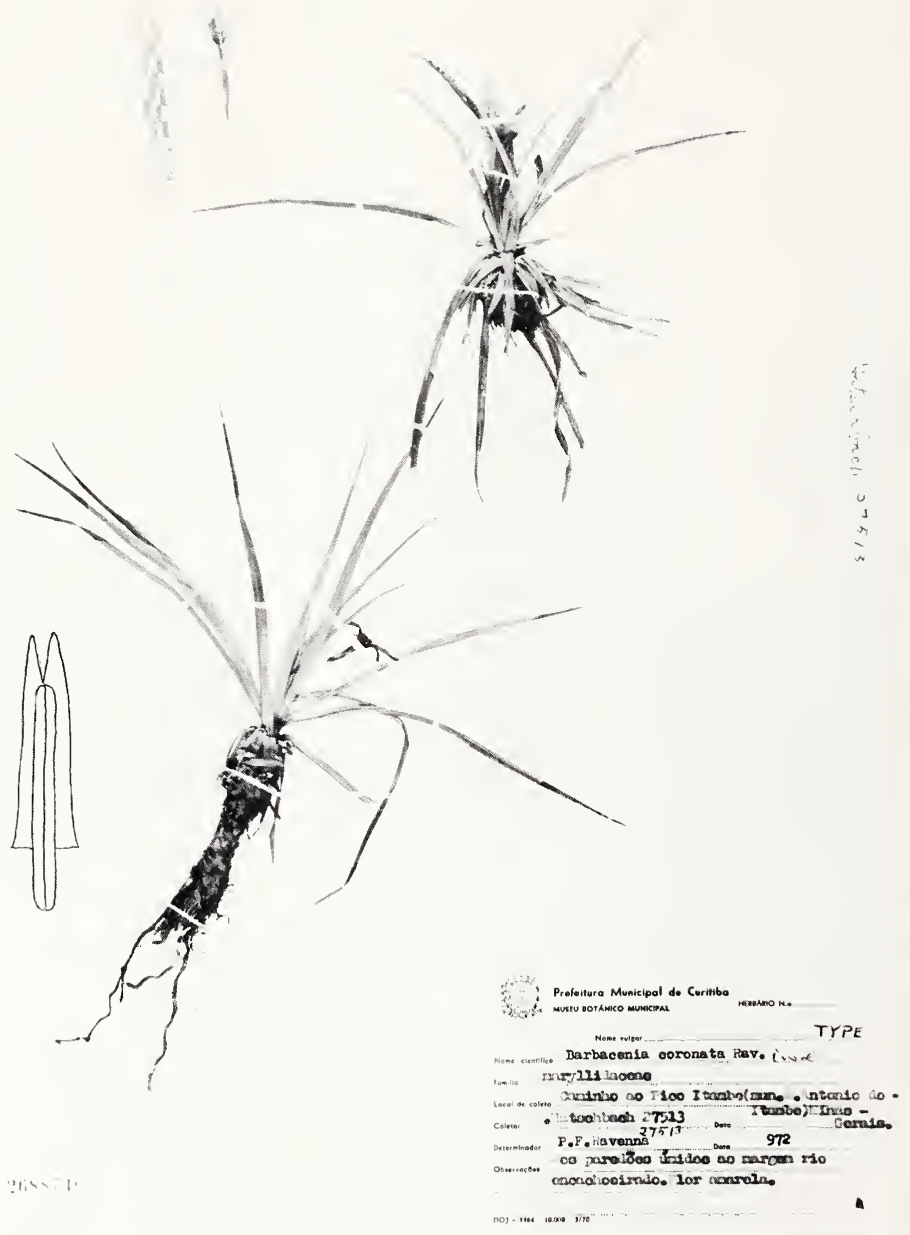

Plate 18.-Barbacenia coronata P. F. Ravenna, new species (Hatschbach 27513). 


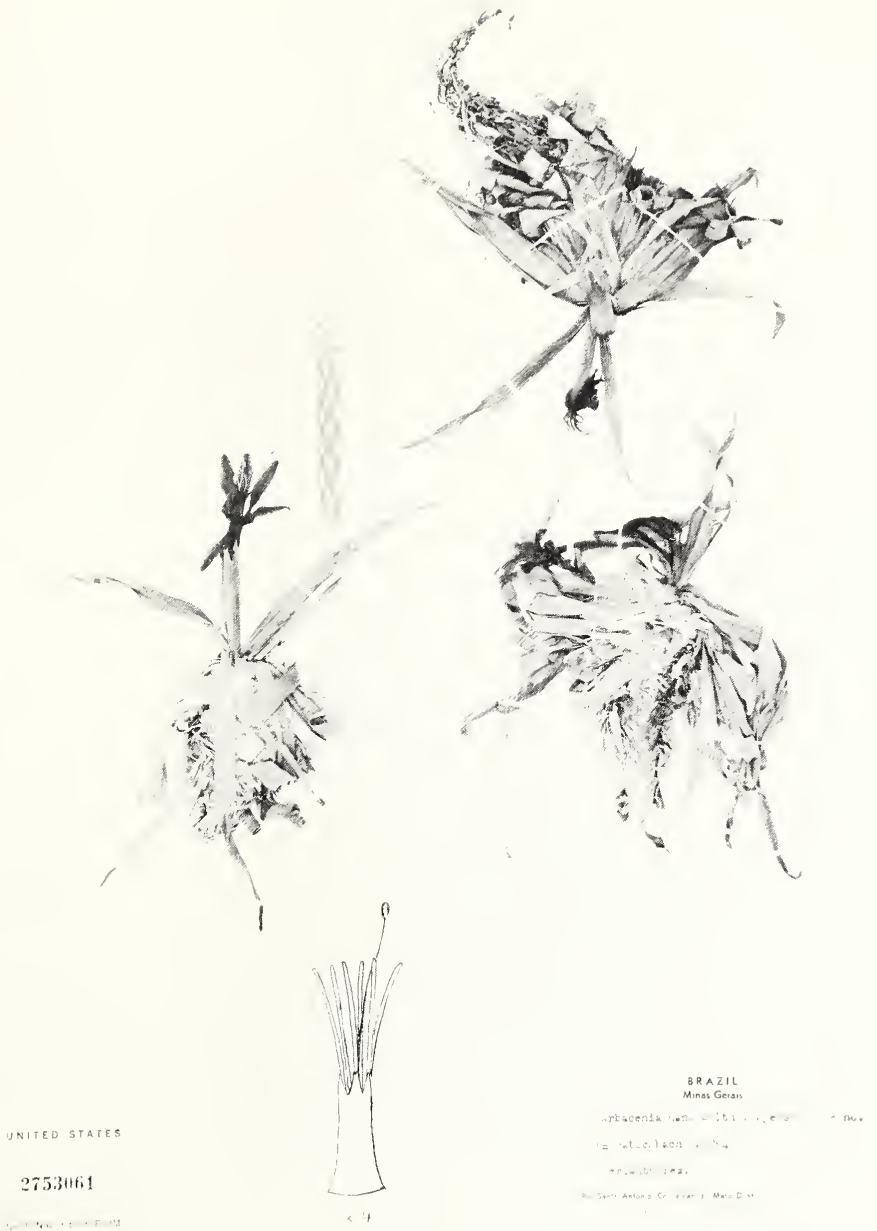

Plate 19.-Barbacenia nana L. B. Smith \& Ayensu, new species (L. B. Smith, Ayensu \& Hatschbach 15973). 

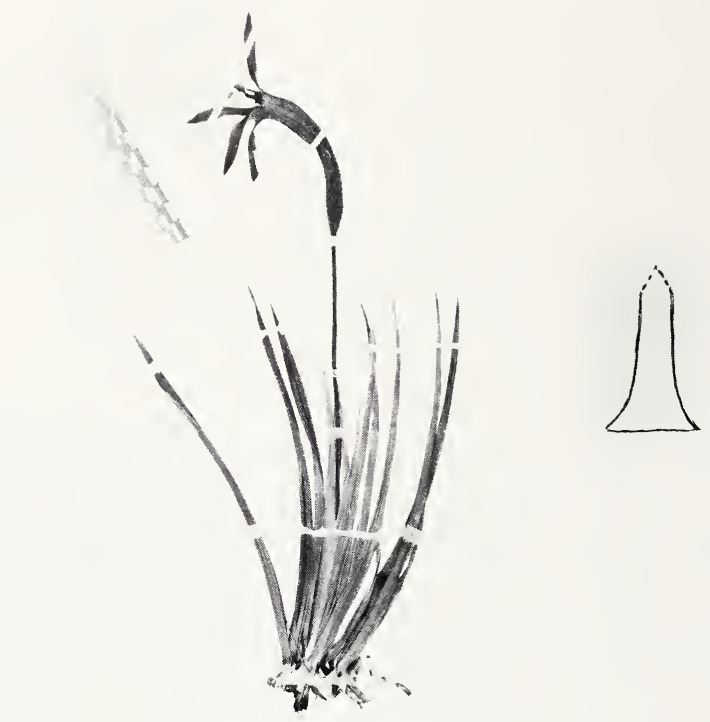

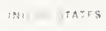

$26,241,37$

Prelestura Municipal de Curitiba

museu sotanico mumicipal

Barbacenca filament ifera

rootso veloziacese LB 5 mith \& Ayen in

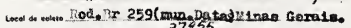

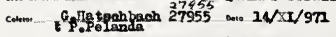

Deturamosos. Por $1400 \mathrm{~m}$

Dof : 3 .

Plate 20.-Barbacenia filamentifera L. B. Smith \& Ayensu, new species (Hatschbach \& Pelanda 27955). 

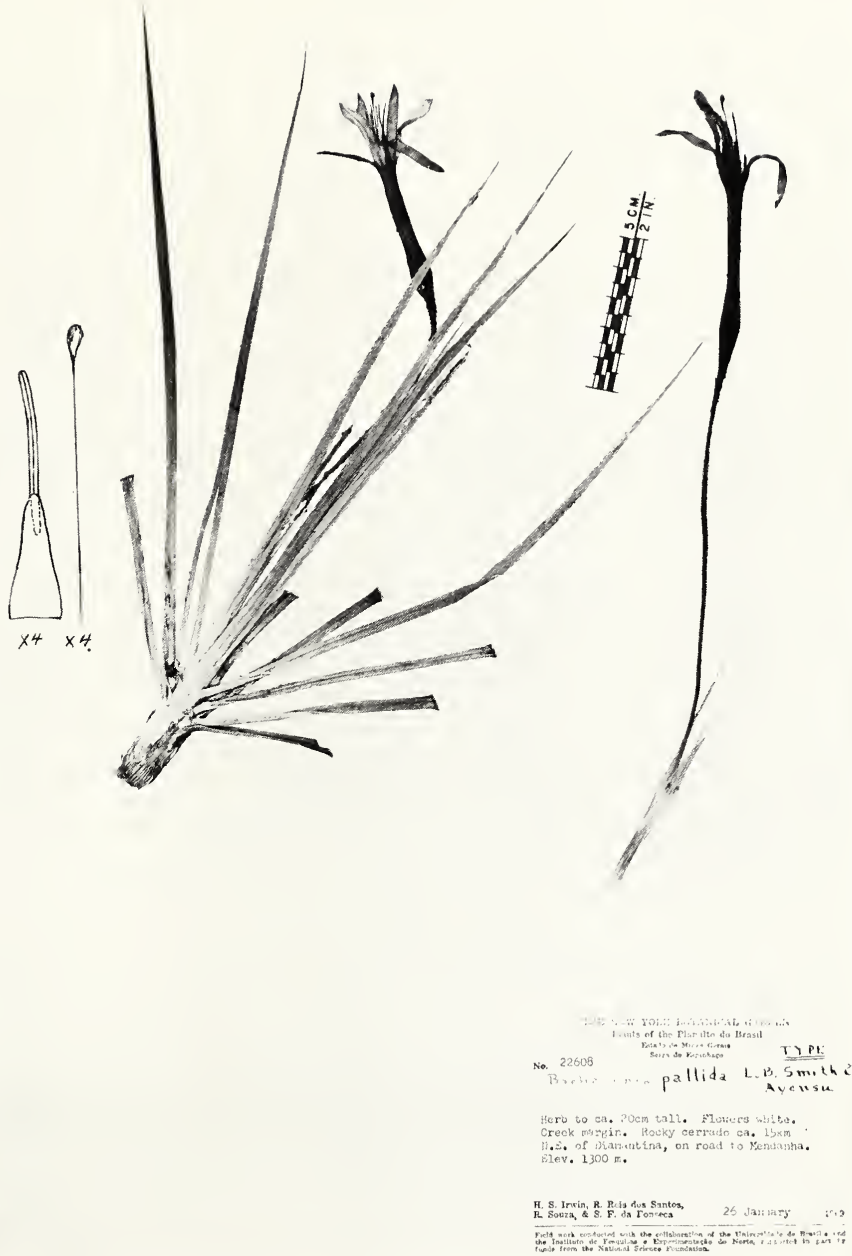

Plate 21.-Barbacenia pallida L. B. Smith \& Ayensu, new species (Irwin et al. 22608). 


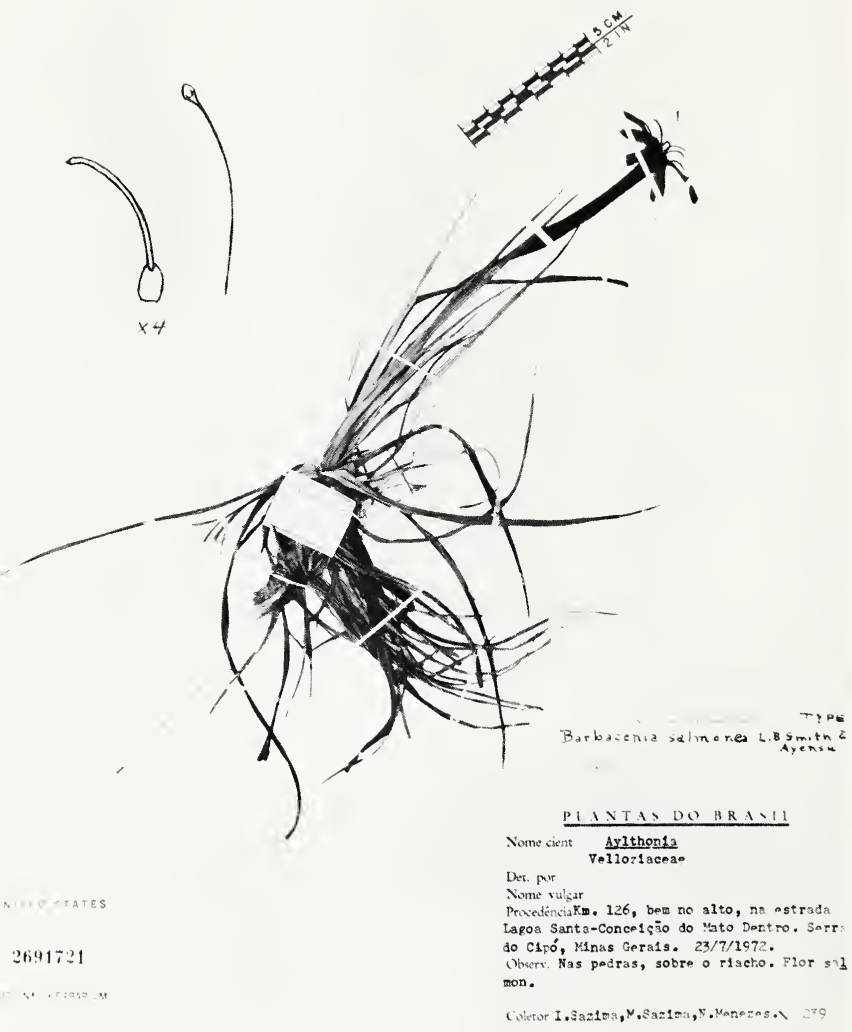

Plate 22.-Barbacenia salmonea L. B. Smith \& Ayensu, new species (Sazima, Sazima \& Menezes 239). 


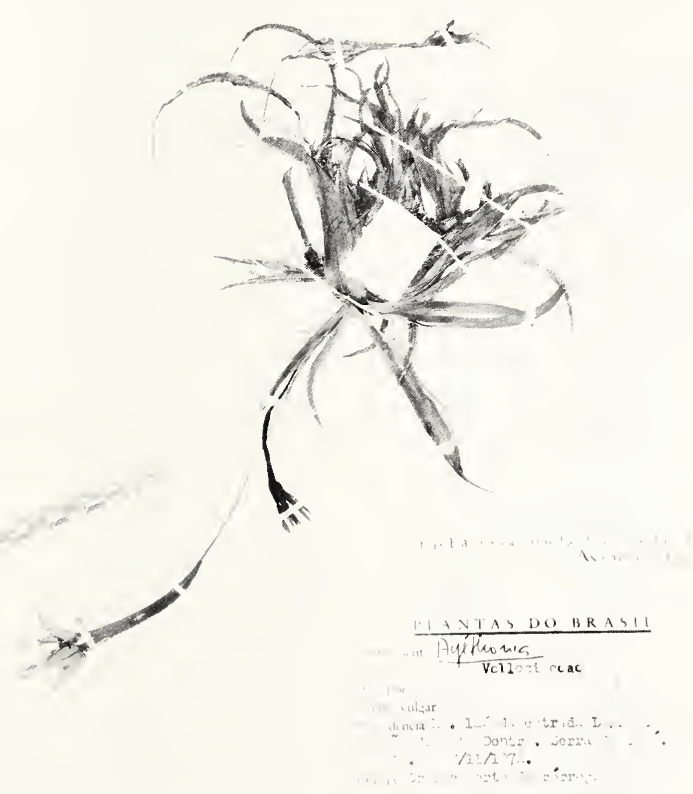




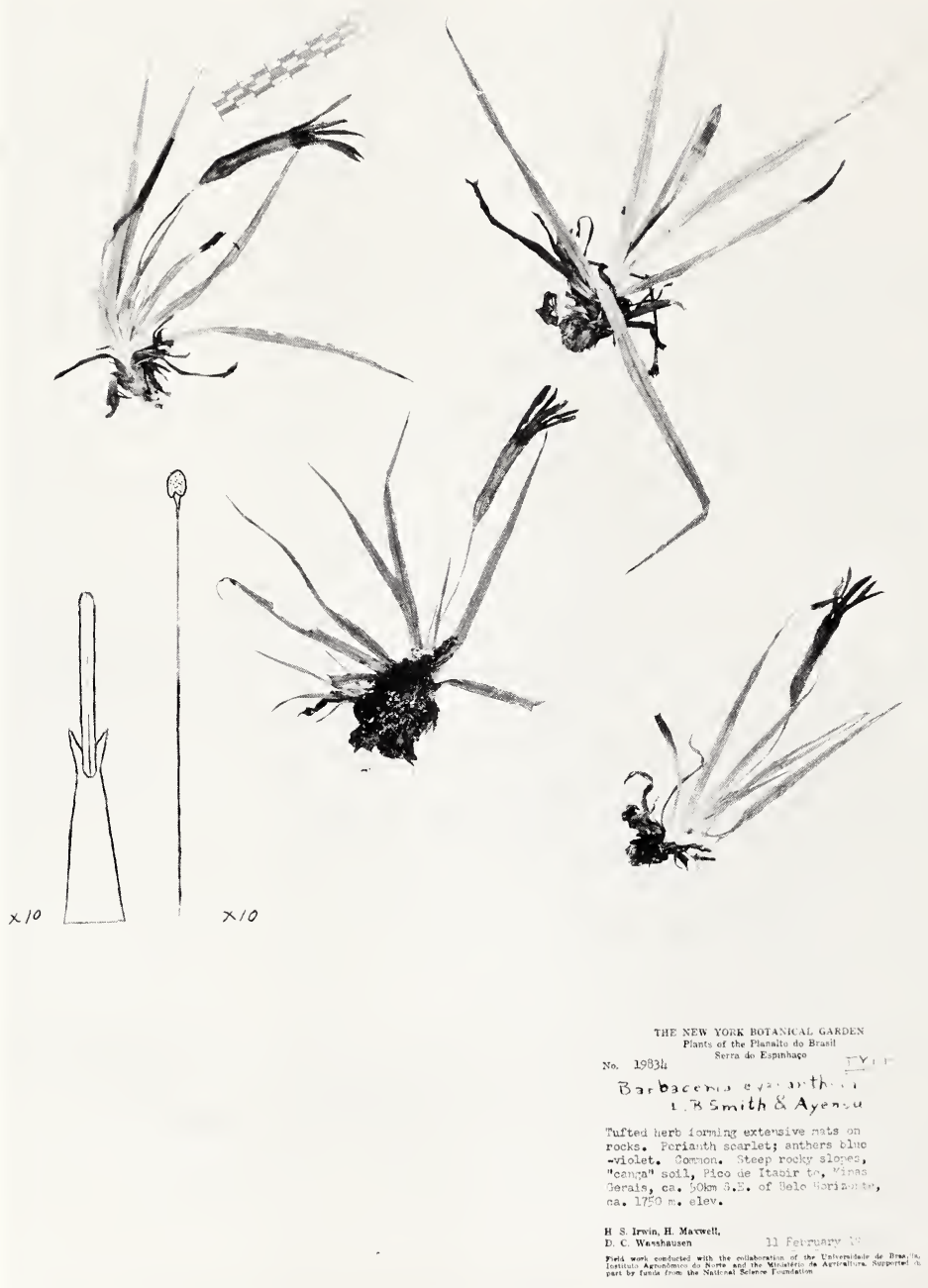

PLATE 24.-Barbacenia cyananthera L. B. Smith \& Ayensu, new species (Irwin, Maxwell or Wasshausen 19834). 


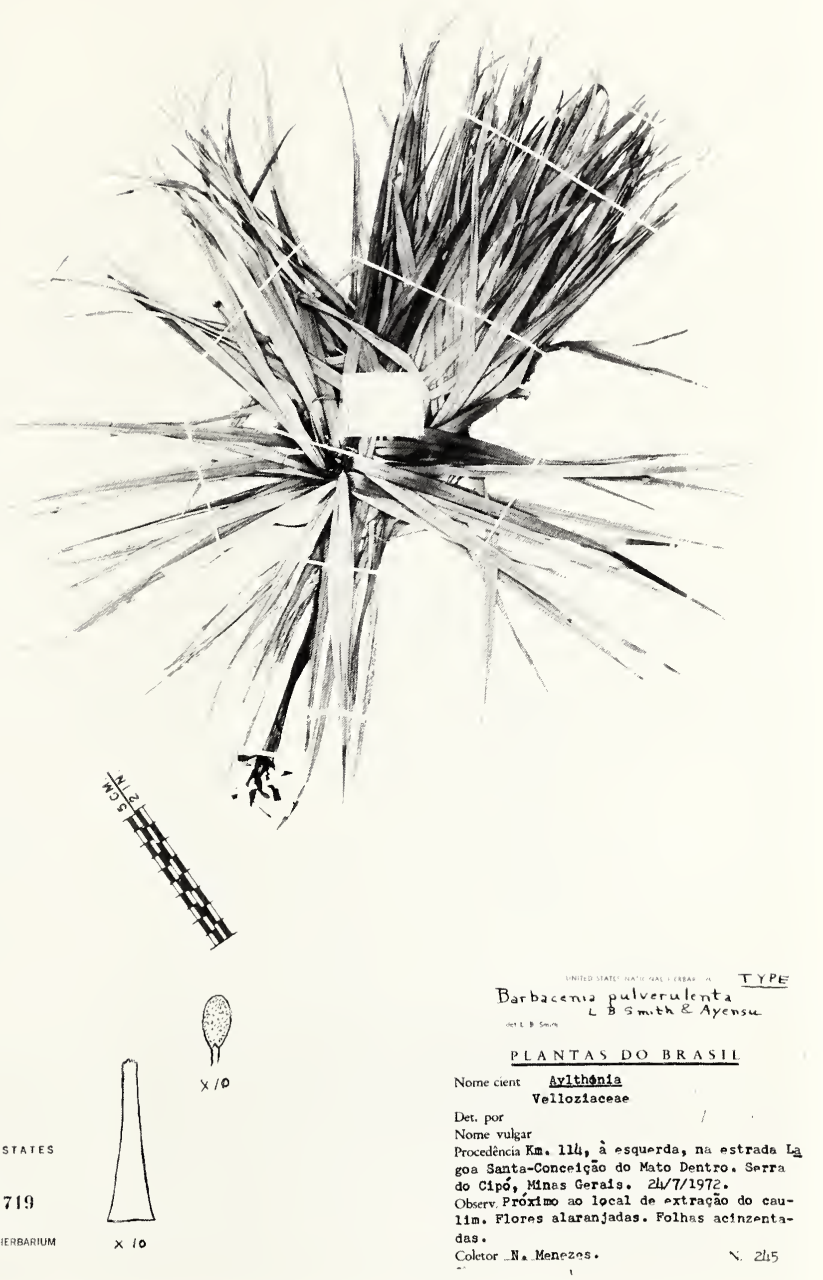

Plate 25.-Barbacenia pulverulenta L. B. Smith \& Ayensu, new species (Menezes 245). 


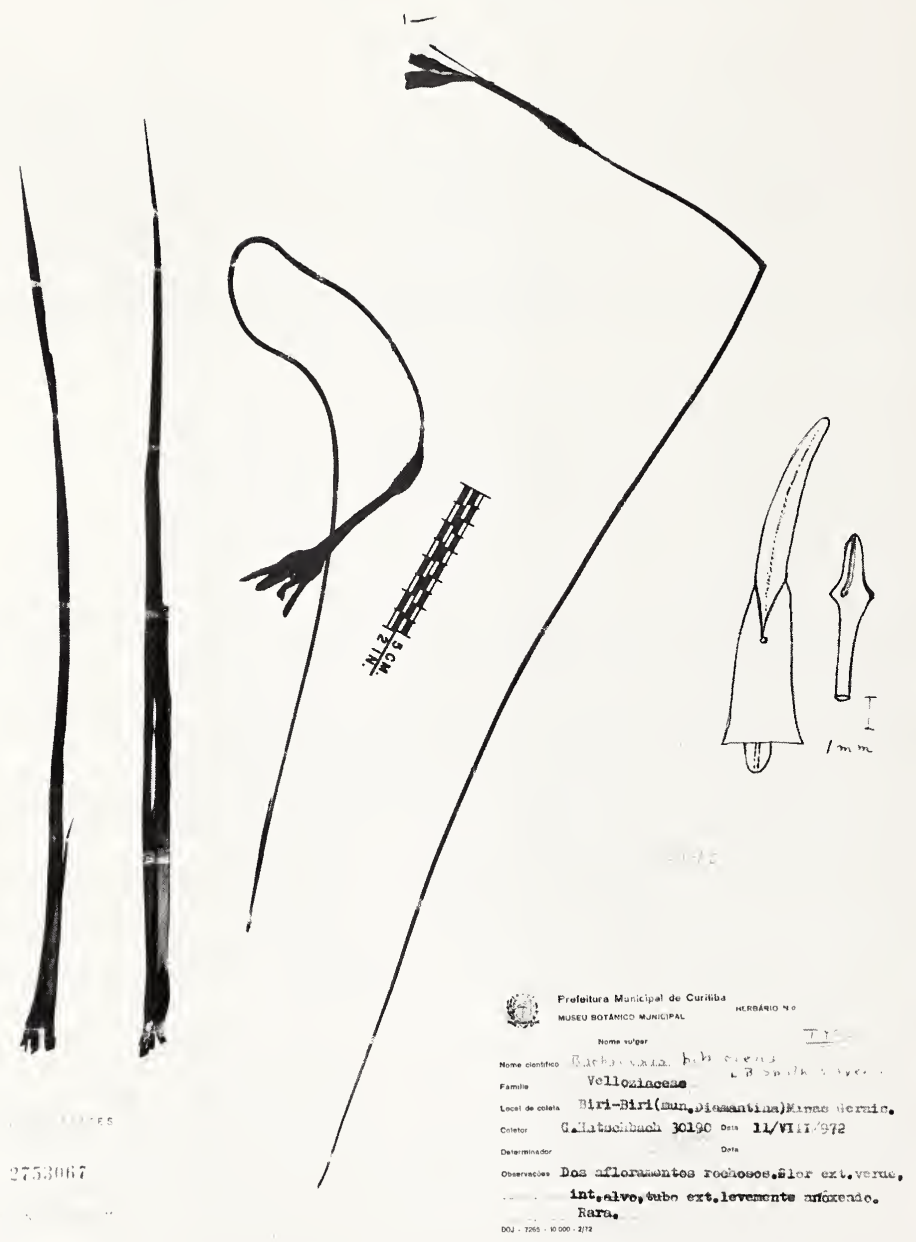

Plate 26.-Barbacenia bibiriensis L. B. Smith \& Ayensu, new species (Hatschbach 30190). 


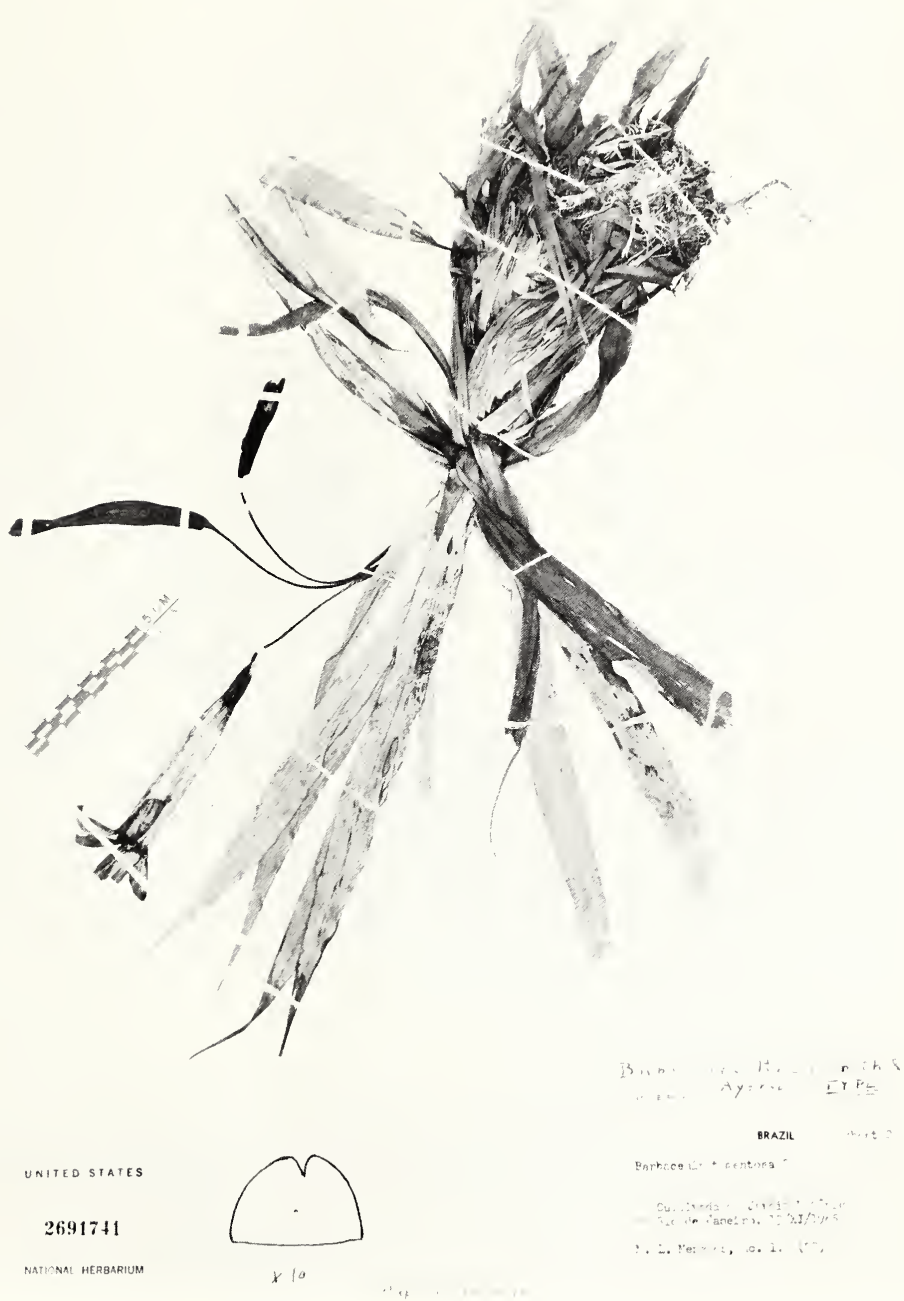

Plate 27.-Barbacenia culta L. B. Smith \& Ayensu, new species (Menezes 1). 


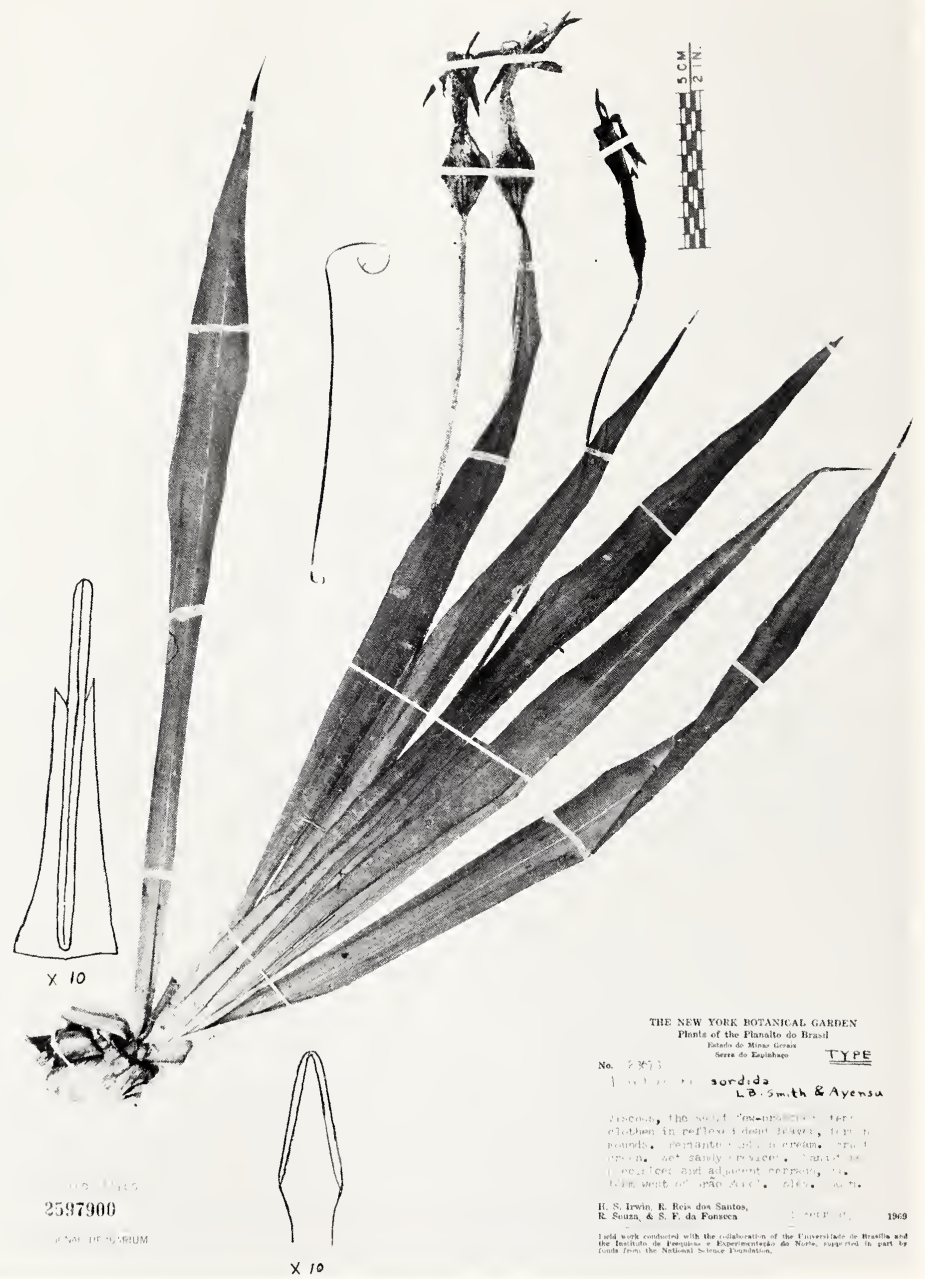

Plate 28.-Barbacenia sordida L. B. Smith \& Ayensu, new species (Irwin et al. 23673). 

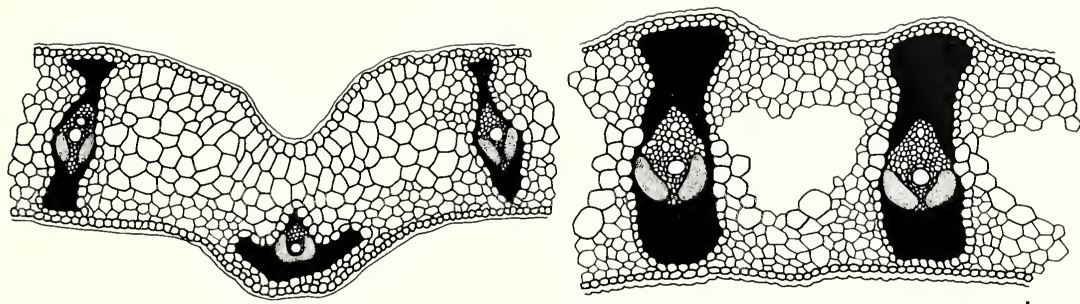

a

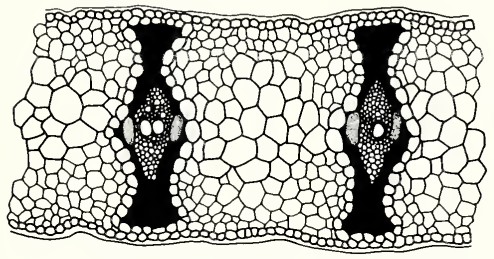

b
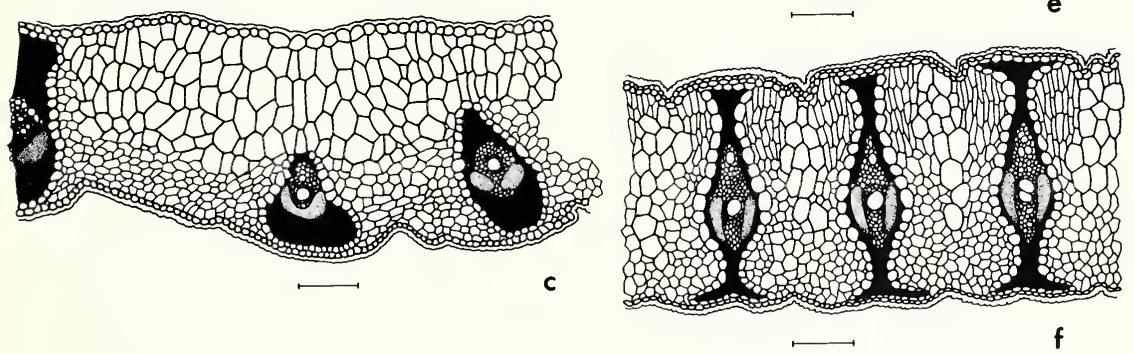

Plate 29.-Leaf anatomy: $a, b$, Barbacenia cylindrica L. B. Smith \& Ayensu, new species (Anderson 7217); $c, d$, Barbacenia spiralis L. B. Smith \& Ayensu, new species (Irwin et al. 27642); $e, f$, Barbacenia pabstiana L. B. Smith \& Ayensu, new species (Hatschbach \& Ahumada 31360). 

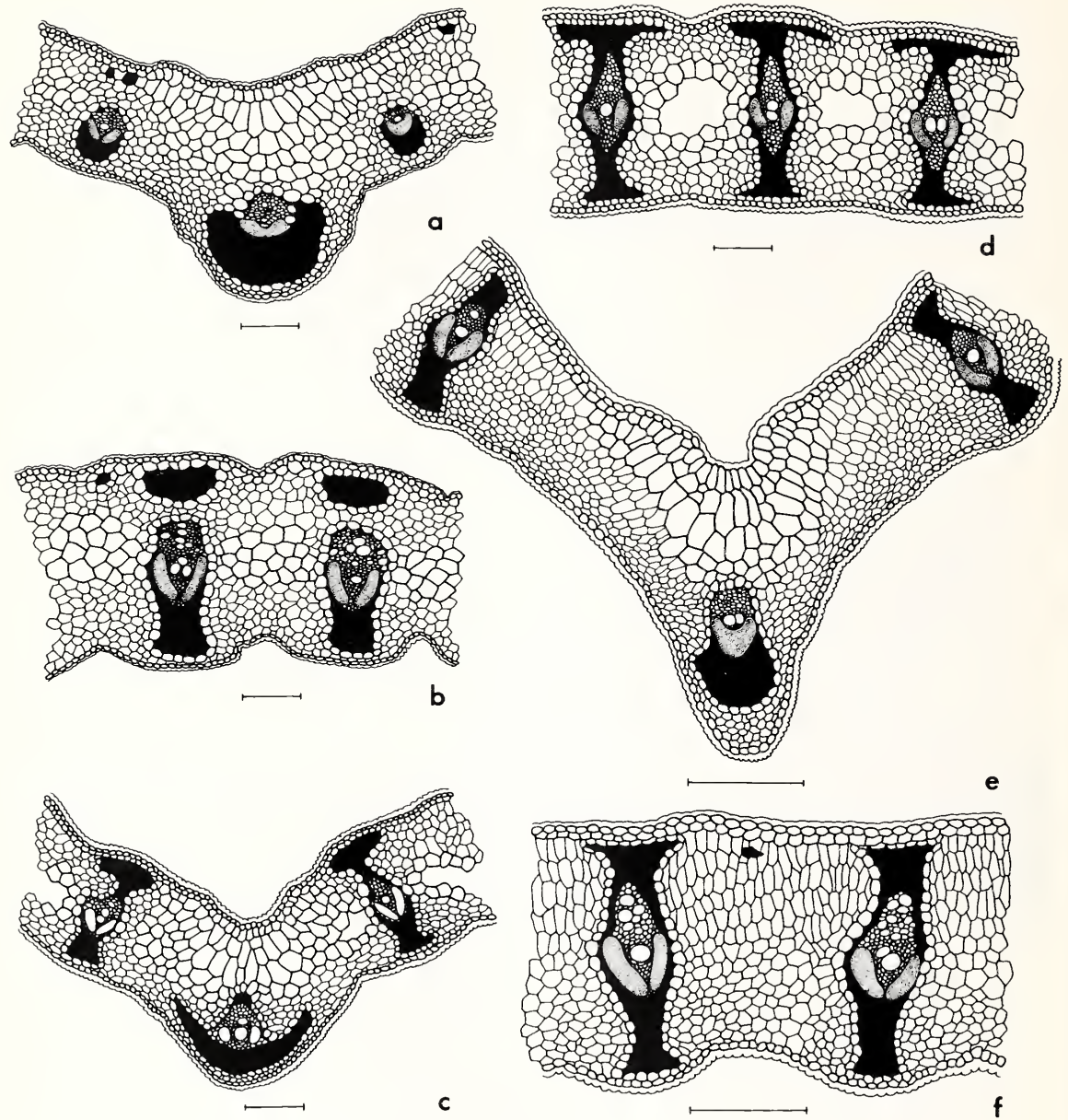

Plate 30.-Leaf anatomy: $a, b$, Barbacenia delicatula L. B. Smith \& Ayensu, new species $(L . B$. Smith, Ayensu \& Hatschbach 15975); $c, d$, Barbacenia hatschbachii L. B. Smith \& Ayensu, new species (Hatschbach 24284); $e, f$, Barbacenia monticola L. B. Smith \& Ayensu, new species (Hatschbach \& Ahumada 31402). 

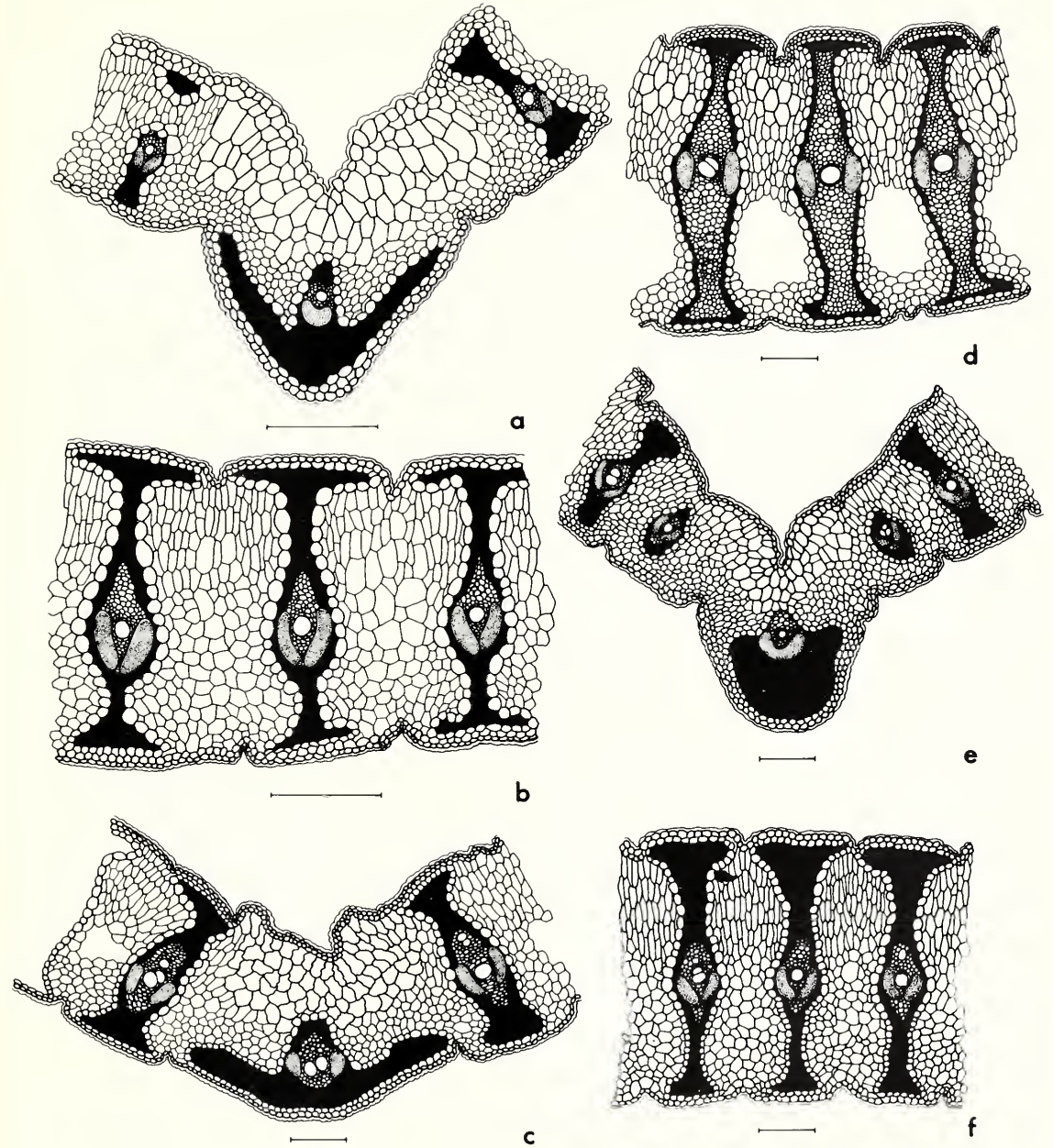

Plate 31.-Leaf anatomy: $a, b$, Barbacenia burle-marxii L. B. Smith \& Ayensu, new species (L. B. Smith \& Ayensu 16014); c, d, Barbacenia spectabilis L. B. Smith \& Ayensu, new species (Oliveira Filho \& Dias 3); $e, f$, Barbacenia andersonii L. B. Smith \& Ayensu, new species (Anderson 6704). 

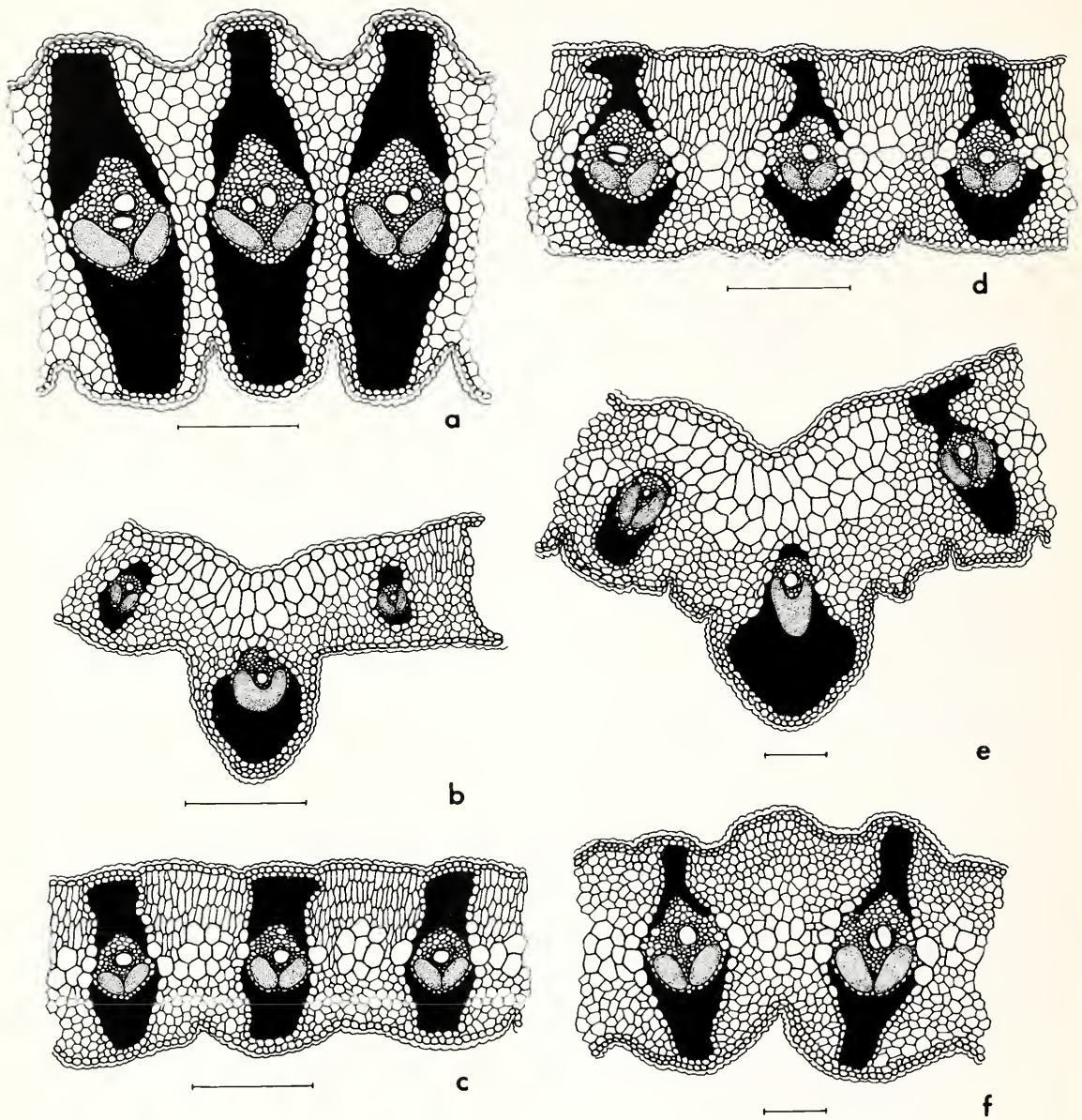

Plate 32.-Leaf anatomy: $a$, Barbacenia nanuzae L. B. Smith \& Ayensu, new species (Menezes 265); b, c, Barbacenia chlorantha L. B. Smith \& Ayensu, new species (Anderson et al. 35488); $d$, Barbacenia saxicola L. B. Smith \& Ayensu, new species (Anderson et al. 35344); e, f, Barbacenia reflexa L. B. Smith \& Ayensu, new species (Maguire et al. 49260). 

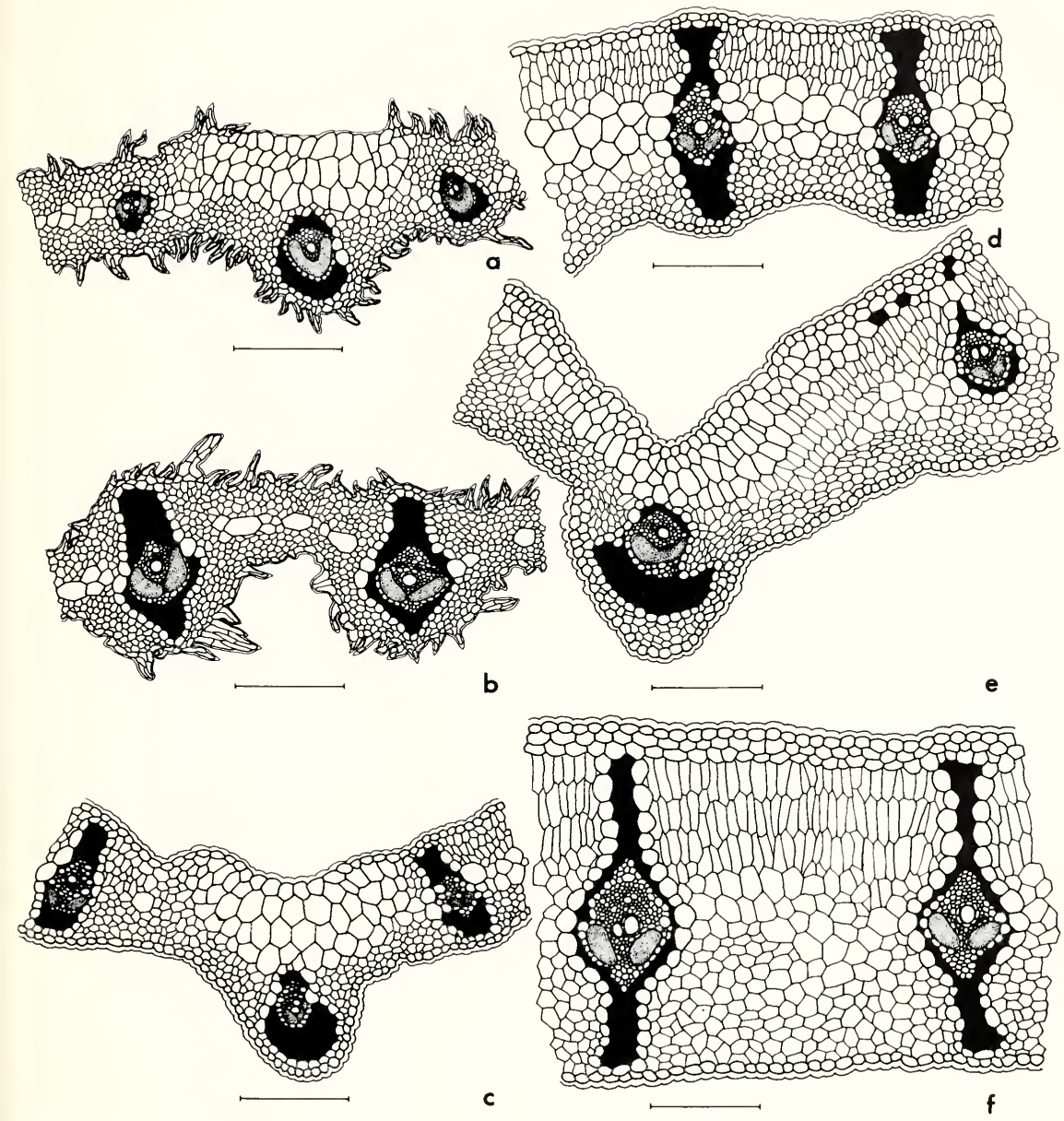

Plate 33.-Leaf anatomy: $a, b$, Barbacenia latifolia L. B. Smith \& Ayensu, new species (Duarte \& Graziela 7884); c, d, Barbacenia umbrosa L. B. Smith \& Ayensu, new species (Irwin et al. 23491); $e, f$, Barbacenia rectifolia L. B. Smith \& Ayensu, new species (Anderson et al. 35674). 


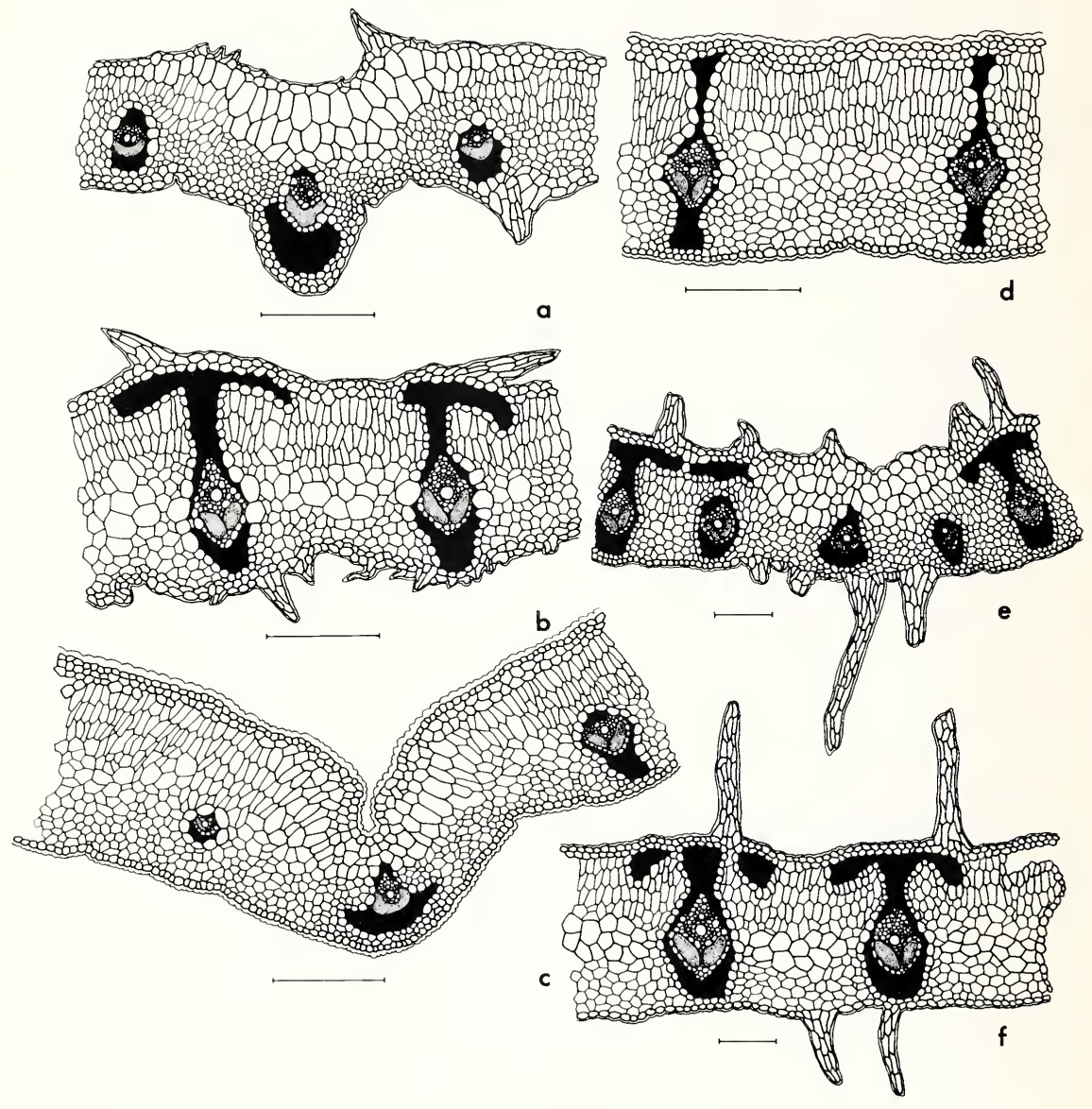

Plate 34.-Leaf anatomy: $a, b$, Barbacenia aurea L. B. Smith \& Ayensu, new species (Irwin et al. 29046); c, d, Barbacenia coronata P. F. Ravenna, new species (Hatschbach 27513); $e$, $f$, Barbacenia nana L. B. Smith \& Ayensu, new species (L. B. Smith, Ayensu, \& Hatschbach 15973). 

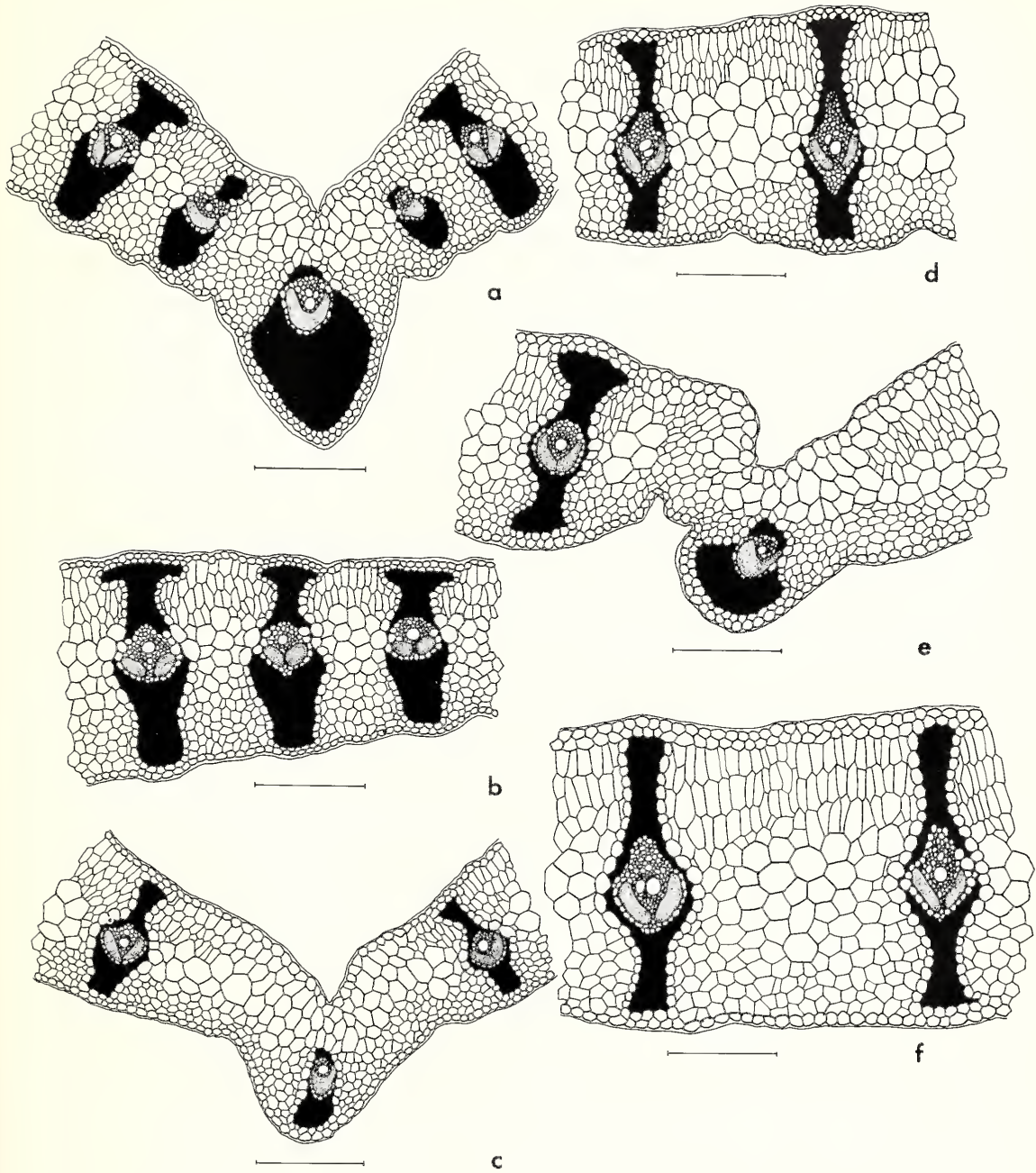

Plate 35.-Leaf anatomy: $a, b$, Barbacenia filamentifera L. B. Smith \& Ayensu, new species (Hatschbach of Pelanda 27955); c, d, Barbacenia pallida L. B. Smith \& Ayensu, new species (lrwin et al. 22608); $e$, f, Barbacenia salmonea L. B. Smith \& Ayensu, new species (Sazima, Sazima do Menezes 239). 


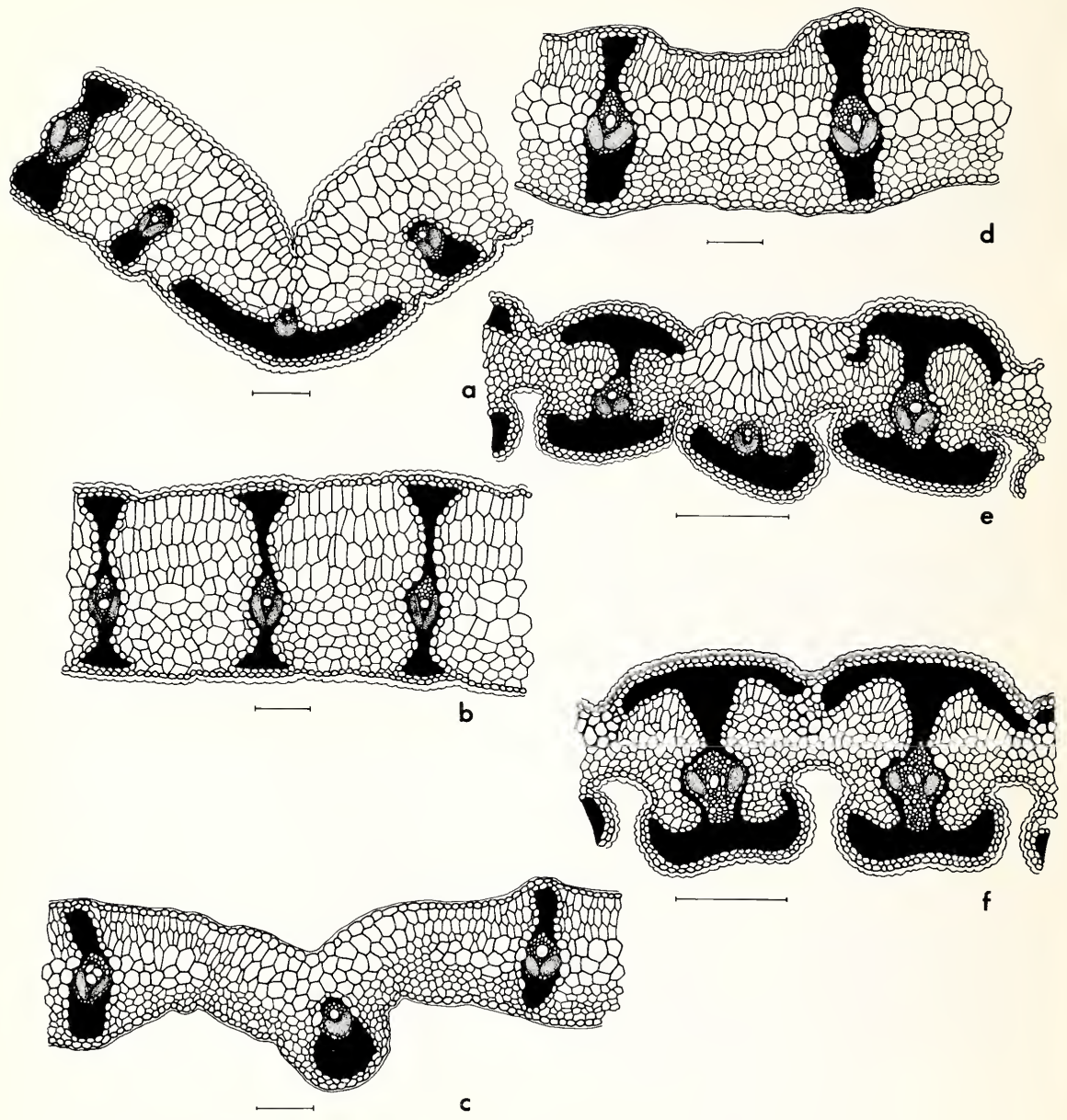

Plate 36.-Leaf anatomy: $a, b$, Barbacenia nuda L. B. Smith \& Ayensu, new species (Semir \& Menezes 282); c, d, Barbacenia cyananthera L. B. Smith \& Ayensu, new species (Irwin, Maxwell \& Wasshausen 19834); $e, f$, Barbacenia pulverulenta L. B. Smith \& Ayensu, new species (Menezes 245). 

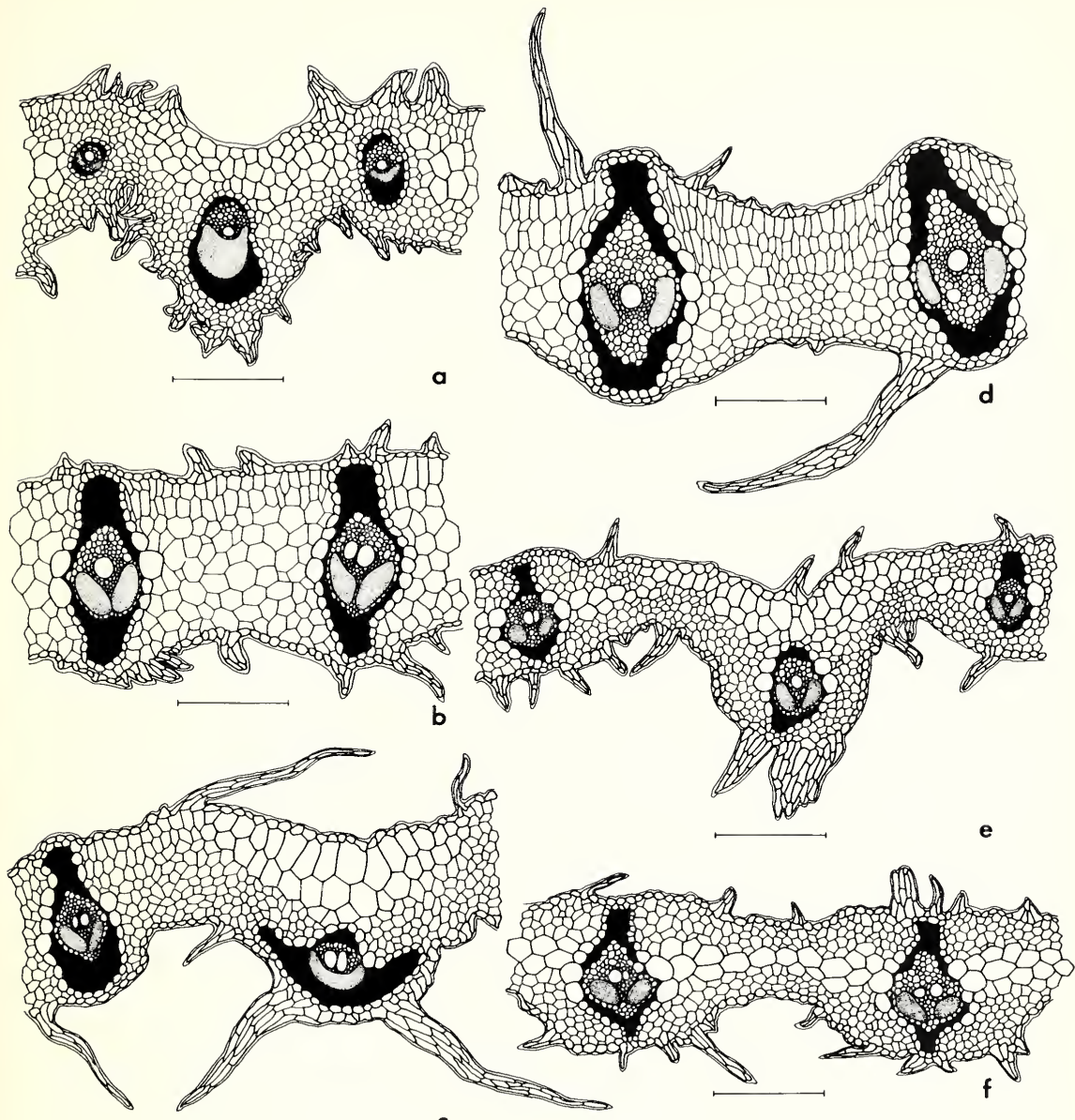

C

Plate 37.-Leaf anatomy: $a, b$, Barbacenia bibiriensis L. B. Smith \& Ayensu, new species (Hatschbach 30190); c, d, Barbacenia culta L. B. Smith \& Ayensu, new species (Menezes 1); e, f, Barbacenia sordida L. B. Smith \& Ayensu, new species (Irwin et al. 23673). 


\section{Index}

(Synonyms and principal entries in italics)

Amaryllideae-Vellozieae, 3

Ayensua uaipanensis, 128

Aylthonia, 1, 4

bahiana, 37

blackii, 36

cuspidata, 33

damaziana, 37

exscapa, 25

gentianoides, 28

leucopoda, 36

luzulifolia, 23

macrantha, 22

magalhaesii, 28

nigrimarginata, 17

paranaensis, 24

rubra, 37

tomentosa, 31

tricolor, 34

viscosissima, 25

Barbacenia, 1, 2, 3, 4, 20

albiflora, 33

alexandrinae, 128

andersonii, 18

aurea, 26

bahiana, 37

beauverdii, 12

bibiriensis, 33

bicolor, 20

blackii, 36

blanchetii, 35

blanchetii, 35

boliviensis, 37

brachycalyx, 11

brasiliensis, 4,20

brevifolia, 16

var. brevifolia, 16

var. recurvata, 16

burle-marxii, 15

caricina, 13

castilloni, 37

celiae, 18

chlorantha, 22

coccinea, 19

conicostigma, 33

coronata, 27

culta, 35

curviflora, 34

cuspidata, 33

cyananthera, 31 cylindrica, 11

damaziana, 37

delicatula, 13

ensifolia, 32, 33

exscapa, 25

filamentifera, 29

flava, 21

var. flava, 21

var. minor, 21

flavida, 15

foliosa, 15

fragrans, 34

fulva, 19

gardneri, 34

gaveensis, 14

gentianoides, $4,27,28$

var. gentianoides, 28

var. magalhaesii, 28

glabra, 30

glauca, 21

glaziovii, 33

globata, 19

glutinosa, 19

goethartii, 16

gounelleana, 17

gracilifiora, 32

gracilis, 128

graminifolia, 24

grisea, 28,29

hatschbachii, 14

hilairei, 28

hirtiflora, 20, 21

ignea, 19

inclinata, 14

involucrata, 18

ionantha, 16

irwiniana, 15

itabirensis, 21

latifolia, 24

leucopoda, 36

lilacina, 20

var. lilacina, 20

var. pallidiflora, 20

longiflora, 34

longiscapa, 14

luzulifolia, 23

macrantha, 22

magalhaesii, 28

mantiqueirae, 16 markgrafii, 19

mollis, 35

var. microphylla, 35

var. mollis, 35

monticola, 14

nana, 27

nanuzae, 20

nigrimarginata, 17

nuda, 30

oxytepala, 28

pabstiana, 12

pallida, 29, 30

paranaensis, 24

plantaginea, 28

polyantha, 19

pulverulenta, 32

purpurea, 4, 18, 129

var. minor, 13

rectifolia, 25

reflexa, 23

riedeliana, 22

rogieri, 13,17

rubra, 36

rubro-virens, 22

rubro-virens, 23, 24

salmonea, 30

saxicola, 23

schidigera, 32

schwackei, 19

sellovii, 22

sessiliflora, 26, 27

seubertiana, 13

sordida, 36

species, 20

spectabilis, 17

spiraiis, 1,11

squamata, 1, 4, 13

stenophylla, 14

tomentosa, 31

tricolor, 34

trigona, 19

tubiflora, 128

uaipanensis, 128, 129

umbrosa, 25

vandelli, 20

vandelli, 20

vargasiana, 38

viscosissima, 24,25

williamsii, 21 
Barbaceniodeae, Subfamily, 4

Barbaceniopsis, 2, 3, 37

boliviensis, 37

humahuaquensis, 37

vargasiana, 38

Campderia, 40

Dasylirion acrotrichum, 128

Nanuza, 38

plicata, 38, 39 (fig.)

Pleurostima, 4

purpurea, 18

Radia, 40, 119

Radia, Section of Vellozia, 119

Radia tubiflora, 128

Veitchia, 4

Vellozia, 1, 2, 3, 38, 40

Section Radia, 119, 129

Section Vellozia, 51

Section Xerophytoides, 48

abietina, 48

alata, 53

albiflora, 93

alexandrinae, 128

aloifolia, 117

aloifolia, 55

alutacea, 69

ambigua, 72

angustifolia, 69

annulata, 125, 126 (fig.)

arenicola, 109

asperula, 62

var. asperula, 62

var. filifolia, 62

bahiana, 99, 101 (fig.)

barbaceniifolia, 77, 80 (fig.)

barbata, 86

bicarinata, 65,66

bicolor, 119

blanchetiana, 97

boliviensis, 37

brachypoda, 55, 56 (fig.)

bradei, 62

brevifolia, 85

var. angustior, 86

var. brevifolia, 85

breviscapa, 77, 79 (fig.)

bulbosa, 99

burle-marxii, 105, 108 (fig.)

cachimbensis, 125

caespitosa, 117, 118 (fig.)

cana, 122

candida, 115, 117

capsulis glabris, scapis hispidis, 128, 129

capsulis scapisque glabris, 53

caput-ardeae, 120 (fig.), 121

caruncularis, $69,75,77$

castanea, 64 (fig.), 65

ciliata, 51

cinerascens, 99,103 circinans, 93

coerulescens, 128

compacta, 72, 75

var. acutiflora, 72

var. obtusiflora, 72

confusa, 88

coronata, 85

costata, 51, 52 (fig.)

crassicaulis, 93

crassirama, 69

crinita, 98 (fig.), 99

crispata, 58

cryptantha, 51

dasypus, 102 (fig.), 103

dawsonii, 122

decidua, 112, 113 (fig.)

declinans, 86

droseroides, 69

duidae, 128

dumitiana, 127

echinata, 109, 112, 115

epidendroides, 88

var. divaricata, 88

var. major, 88

exilis, 103, 105, 106 (fig.)

fibrosa, 58, 59 (fig.)

fimbriata, 77

flavicans, 91

froesii, 97

fruticosa, 75

gardneri, 91

geotegens, 115, 116 (fig.)

glabra, 40, 53

glandulifera, 117, 119

glauca, 91

var. cujabensis, 95

var. genuina, 91

glaziovii, 99

glochidea, 91

gracilis, 88

graminea, 53

granulata, 62, 63 (fig.)

grao-mogulensis, 85

grisea, 103, 105, 107 (fig.)

hamosa, 91

hatschbachii, (front.), 58, 61 (fig.)

hemisphaerica, 105

hirsuta, 122

hypoxoides, 97

incurvata, 51,55

intermedia, 82,83 (fig.)

irwinii, 69

laevis, 53

lanata, 122, 124 (fig.)

lappa, 77

leptopetala, 88,89 (fig.)

leptopetala, 86

leptophylla, 129

leucanthos, 125

lilacina, 109, 110 (fig.) lithophila, 127

macarenensis, 127

macedonis, 53

machrisiana, 122

macrantha, 129

macrosiphonia, 128

maculata, 122, 123 (fig.)

magdalenae, 91

maguirei, 121

marcescens, 86

var. marcescens, 86

var. minor, 88

maritima, 117

markgrafii, 119

martiana, 72

maudeana, 121

maxillarioides, 51

metzgerae, 117

minima, 51

modesta, 77, 78 (fig.)

mollis, 119

nanuzae, 114 (fig.), 115

nivea, 94 (fig.), 95

nuda, 91, 92 (fig.)

ornata, 62

panamensis, 125

papillosa, 62

patens, 75, 76 (fig.)

phalocarpa, 53

phantasmagorica, 125

pilosa, 86, 87 (fig.)

piresiana, 62

pleurocarpa, 51

plicata, 38

pterocarpa, 67, 68 (fig.)

pulchra, 99

pumila, 99, 100 (fig.)

punctulata, 58, 60 (fig.)

pusilla, 55

ramosissima, 65

resinosa, 69, 70 (fig.)

rhynchocarpa, 127

riedeliana, 125

scabra, 129

scabrosa, 103, 104 (fig.)

scoparia, 48, 50 (fig.)

sellovii, 53, 54 (fig.)

seubertiana, 95, 96 (fig.)

sincorana, 55, 57 (fig.)

spiralis, 88

squalida, 85

squamata, 13, 91, 129

stipitata, 109, 111 (fig.)

streptophylla, 86

subalata, 80,81 (fig.)

subscabra, 129

sulphurea, 93

swallenii, 99

taxifolia, 51

tenella, 53 
teres, 69, 71 (fig.)

tertia, 117

tomeana, 82,84 (fig.)

tomentosa, 129

torquata, 72, 73 (fig.)

tragacantha, 48, 49 (fig.)

triquetra, 38

tubiflora, 127

uaipanensis, 129

uleana, 121, 122 (fig.)

variabilis, $55,69,72$

var. tuberculata, 69

var. variabilis, 69

variegata, 90 (fig.), 91 velutinosa, 125

verruculosa, 58

vestita, 119

viannae, 80

virgata, 51

wasshausenii, 74 (fig.), 75

wettsteinii, 69

Velloziaceae, 1, 2

Vellozieae, 2

Vellozioideae, Subfamily, 38

Visnea, 4, 20

Xerophyllum sabadilla, 129

Xerophyta, 1, 3, 38, 40

abietina, 48 boliviensis, 37

cinerascens, 103

minima, 51

plicata, 38

selloi, 53

sellovii, 53

taxifolia, 51

tragacantha, 48

triquetra, 38

vargasiana, 38

Xerophytoides, Section of Vellozia, 48 


\section{Publication in Smithsonian Contributions to Botany}

Menuscipts for serial publications are accepted by the Smithsonian Institureden Press ku

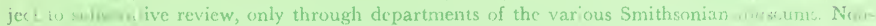
Sunthsmum authors should address inquiries to the appropriate department. If sultirissinn 7 invited, the iniliswing format requirements of the Press will govern the preparation of copy.

Cupj riss be typewritten double-spaced, on one side of standard white bond paper, wit $11 / 2$ " top ant if $f 1$ uargins, subruitted in ribbon copy with a carbon or duplicate, and accompa nicd by the signal artwork. Duplicatc copies of all material, including illustrations, should be retained by the autior. There may be sevcral paragraphs to a pagc, but each page should begin with a ncw naragrapi Number consecutively all pages, including title page, abstract, text, literature cited, legends, and tables. The minimum length is 30 pages, including typescript and illus trations.

The title should be complete and clear for easy indexing by abstracting services. Taxonomit titles will carry a final line indicating the higher catcgories to which the taxon is referable: "(Leguminosae: Faboideae)." Include an abstract as an introductory part of the text. Identify the author un the first page of text with an unnumbered footnote that includes his professional mailing address. A table of contents is optional. An index, if required, may be supplied by the author when he returns page proof.

Two headings are used: (1) text heads (boldface in print) for major sections and chapters and (2) paragraph sideheads (caps and small caps in print) for subdivisions. Further hcadings may be worked out with the editor.

In taxonomic keys, for easy reference, number the taxa and their corresponding headings throughnut the text; do not incorporate page references in the key.

In synonymy, use the long form (taxon, abbreviated author, abbreviated jourual or book title, volume, part if necessary, page, year) with no refcrence at the end of the paper under "Literature Cited" or use the short form (taxon, author, date: page) with a full reference at the end of the paper under "Literature Cited." Begin each taxon at the left margin with subsequent lines indented about three spaces. Within an entry, use a period-dash (.- ) to separate each supplementary reference. Enclose with square brackets any annotation in, or at the end of, the entry. For references within the text, use the author-date system: "(Jones, 1910)" and "Jones (1910)." If the reference is expanded, abbrcviate the data: "Jones (1910:122, pl. 20: fig. 1)."

Simple tabulations in the text (e.g., colimns of data) may carry headings or not, but they should not contain rules. Formal tables must be submitted as pages separate from the text, and each table, no matter how large, should be pasted up as a single sheet of copy.

Use the metric system instcad of, or in addition to, the English system.

Illustrations (line drawings, maps, photographs, shaded drawings) can be intermixed throughout the printed text. They will be termed Figures and should be numbered consecutively; however, if a group of figures is treated as a single figure, the components should be indicated by lowercase italic letters on the illustration, in the legend, and in text references: "Figure $9 b$." If illustrations (usually tone photographs) are printed separately from the text as full pages on a different stock of paper, they will be termed Plates, and individual components should be lettered (Plate $9 b$ ) but may be numbered (Plate 9: figure 2). Never combine the numbering system of text illustrations with that of plate illustrations. Submit all legends on pages separate from the text and not attached to the artwork. An instruction booklet for the preparation of illustrations is available from the Press on request.

In the bibliography (usually called "Literature Cited"), spell out book, journal, and article titles, using initial caps with all words except minor terms such as "and, of, the." For capitalization of titles in foreign languages, follow the national practice of each language. Underscore (for italics) book and journal titles. Use the colon-parentheses system for volume, number, and page citations: "10(2):5-9." Spell out such words as "figures," "plates," "pages."

For free copies of his own paper, a Smithsonian author should indicate his requirements on "Form 36" (submitted to the Press with the manuscript). A non-Smithsonian author will receive 50 free copies; order forms for quantities above this amount with instructions for payment will be supplied when page proof is forwarded. 
